\title{
Signal Enhancement Applied to Pulse Transit Time Measurement
}

\author{
by
}

\section{Xiaochuan He, M. A. Sc.}

\author{
A thesis submitted to \\ the Faculty of Graduate and Postdoctoral Affairs \\ in partial fulfillment of the requirements for the degree of \\ Doctor of Philosophy \\ in
}

Electrical and Computer Engineering

Ottawa-Carleton Institute for Electrical and Computer Engineering

Department of Systems and Computer Engineering

Carleton University

Ottawa, Ontario

December, 2015

Copyright (C) 2015- Xiaochuan He 


\section{Abstract}

High arterial blood pressure, or hypertension, is a major risk factor for cardiovascular diseases. Conventional noninvasive methods for estimating the arterial blood pressure rely on a brachial cuff that is placed around the upper arm. The cuff is inflated to a pressure that exceeds the systolic blood pressure. The heart pulse is monitored while the cuff is slowly deflated. The pressure at which the heart pulse sound can be detected corresponds to the systolic pressure and the pressure at which the heart pulse sound can no longer be detected corresponds to the diastolic pressure. This approach cannot be used on a continuous basis and has many disadvantages, including the fact that the system is cumbersome and causes discomfort to the patient, which may affect their blood pressure value.

This thesis proposes two approaches to accurately measure the pulse transit time (PTT). The PTT can be used to estimate arterial blood pressure variations noninvasively, on a continuous basis without the use of a cuff. The correlation between the arterial blood pressure and PTT are verified using bio-signals from the MIMIC II database.

The first approach for measuring the PTT relies on the electrocardiogram (ECG) and photo-plethysmograph (PPG) signals. Algorithms based on the empirical mode decomposition (EMD) method and the adaptive filtering techniques are proposed to enhance the corrupted ECG signal. Gaussian-based curve fitting algorithms are proposed to model and extract the embedded characteristic parameters from the original PPG signal. 
The second approach relies on a novel framework: the Eulerian video magnification (EVM). The color component of the video signal is amplified to reveal subtle change in the skin color. These changes correspond to the blood flow into the arteries. Simulations and experiments are conducted to validate the proposed framework.

The results demonstrate that the enhanced ECG and PPG signals can improve the accuracy of the PTT in estimating the arterial blood pressure. The EVM method can capture the characteristics of the wrist pulse signal and detect the heart pulse on different areas of the human skin. This paves the way towards the usage of cameras for continuous arterial blood pressure monitoring. 


\section{Acknowledgements}

I deeply would like to express my sincere thanks to my supervisors Professor Rafik A. Goubran and Professor Xiaoping P. Liu. They have provided me with fruitful ideas and invaluable advices. Their support and encouragement to my research is the key element of all the achievements and publications.

I would like to thank my colleagues in the DSP lab and all the staffs from my department for sharing their knowledge and offering their generous help. A special thanks to my committee members for their valuable comments on my work.

I would like to especially thank my parents, my husband Yanwei and my son Samuel for always being at my side. Thanks all my treasured friends for their love and accompany. 


\section{Table of Contents}

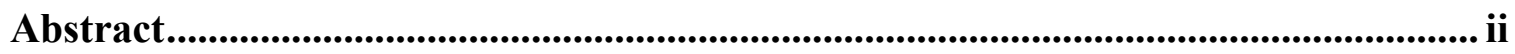

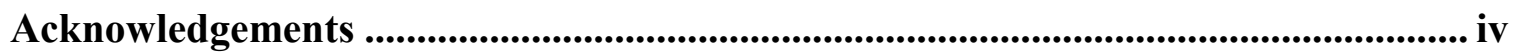

Table of Contents ............................................................................................................................ v

List of Figures............................................................................................................................. xi

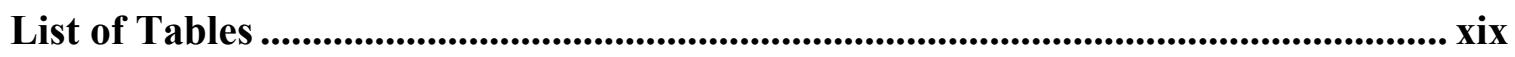

List of Appendices.................................................................................................................... xxi

List of Acronyms ........................................................................................................ xxii

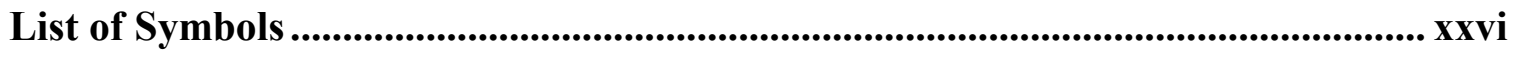

1 Introduction......................................................................................................................... 1

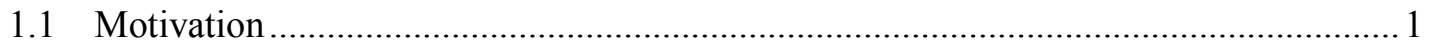

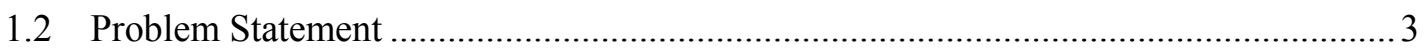

1.3 Thesis Objectives and Summary of the Results ........................................................ 4

1.4 Outline of the Thesis .......................................................................................

1.5 Statement of Originality and Main Contributions …………………………………..

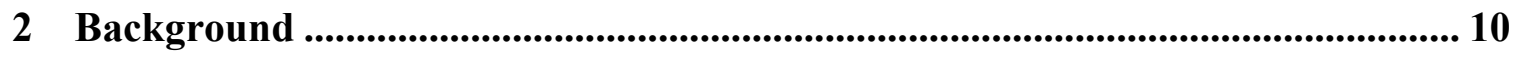


2.1 Cardiovascular System

2.2 Blood Pressure Measurement Techniques .16

2.2.1 Validation Standards for Blood Pressure Measurement 16

2.2.2 Direct Measurement. 17

2.2.3 Occlusive Measurement. .17

2.2.4 Semi-Occlusive Measurement 21

2.2.5 Pulse Wave Velocity

2.3 Pulse Transit Time Technique. .26

2.4 Electrocardiography .29

2.4.1 ECG Morphology .29

2.4.2 ECG Measurement 31

2.4.3 Noises in ECG 32

2.4.4 ECG Database .33

2.4.5 ECG De-noise Algorithms. .34

2.5 Photoplethysmography. 37

2.5.1 Principle of PPG .38

2.5.2 PPG Measurement .39

2.5.3 Peak Shifting Phenomena 40 
2.6 Eulerian Video Magnification 44

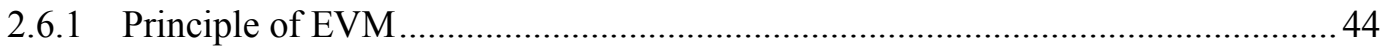

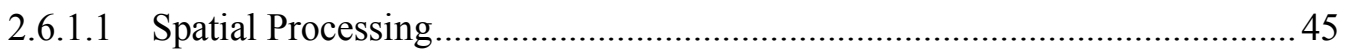

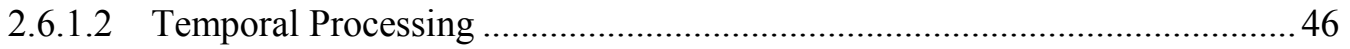

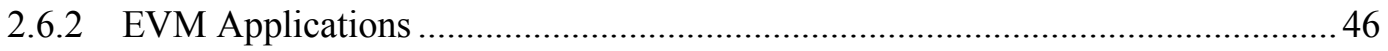

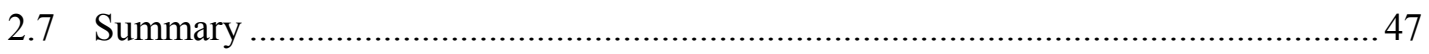

3 ECG Enhancement using EMD-based Algorithm ............................................... 48

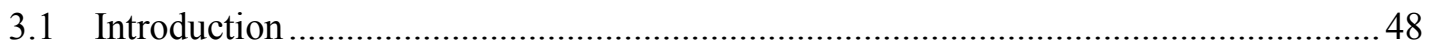

3.2 Overview for EMD-based Algorithm and Adaptive Technique ............................... 49

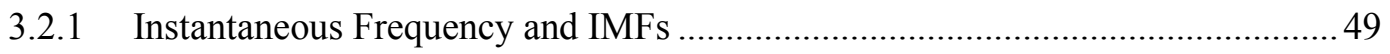

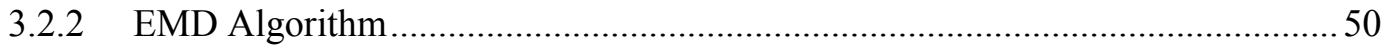

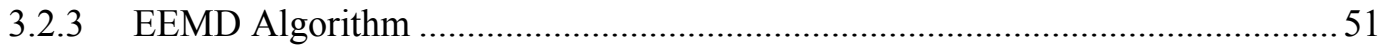

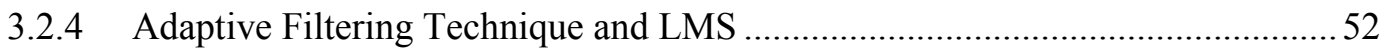

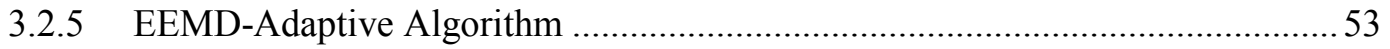

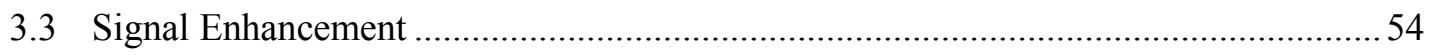

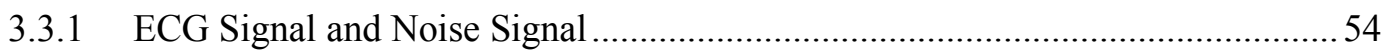




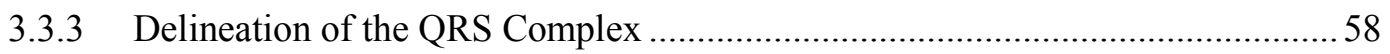

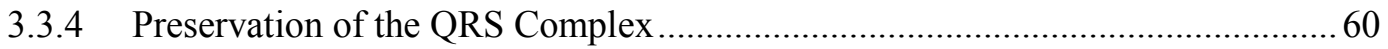

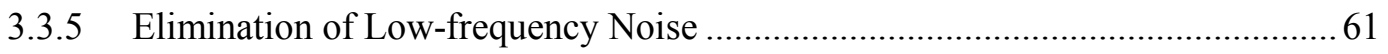

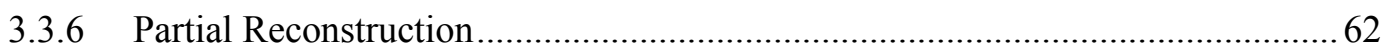

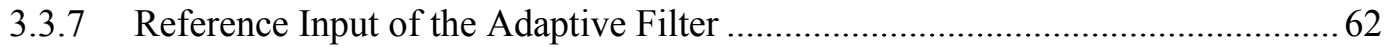

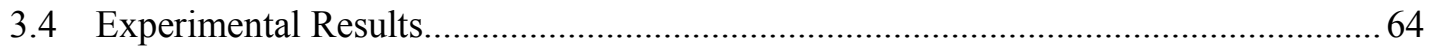

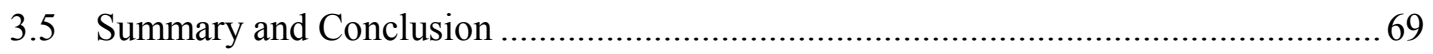

4 PPG Signal Description, Modeling, and Peak Detection....................................... 71

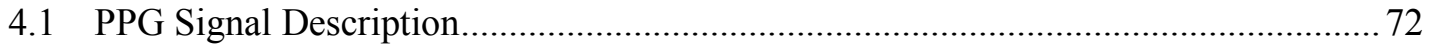

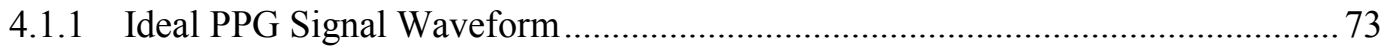

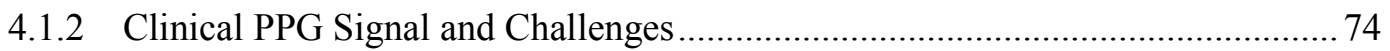

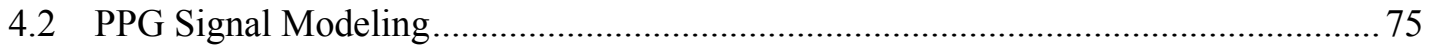

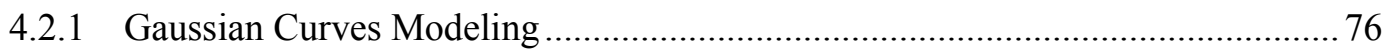

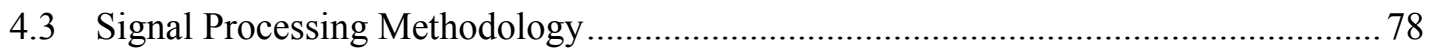

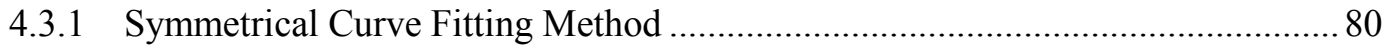

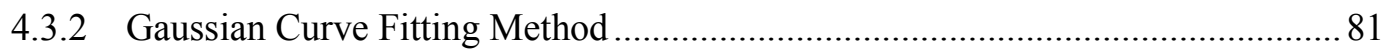

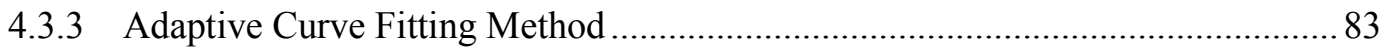


4.4 The Procedure of Secondary Peak Detection. .84

4.4.1 Secondary Peak Detection 84

4.5 Detection Ability Demonstration of Proposed Methods .87

4.5.1 Different Scenarios 87

4.5.2 Curve Fitting Methods .93

4.6 Primary Peak Location Analysis......

4.7 Summary and Conclusion 102

5 Correlation between Arterial Blood Pressure and Pulse Transit Time 104

5.1 Signal Analysis 105

5.2 BP-PTT Relation

5.3 BP and Adjusted PTT

5.4 Summary and Conclusion 119

6 Wrist Pulse Measurement and Analysis Using Eulerian Video Magnification Framework . 120

6.1 Introduction 120

6.2 Overview of the Eulerian Video Magnification Framework.

6.2.1 Framework of Eulerian Video Magnification 122

6.2.2 Eulerian Color Magnification 125 


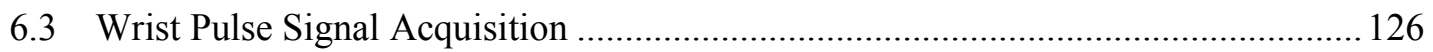

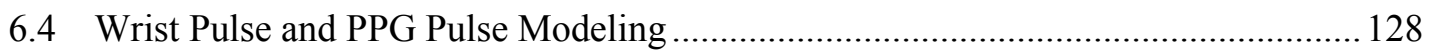

6.5 Characteristic Parameter Comparison ........................................................................ 130

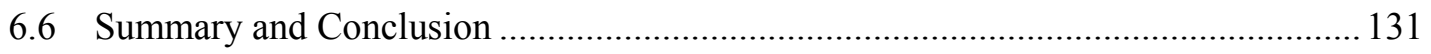

7 PTT Measurement Using Eulerian Video Magnification Framework .............. 133

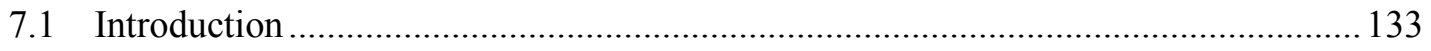

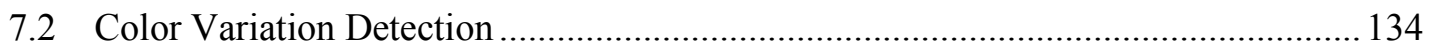

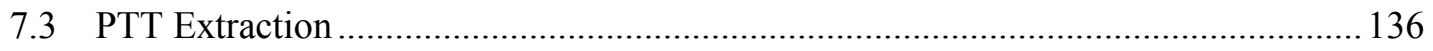

7.4 Experiments Using Eulerian Color Magnification ................................................... 138

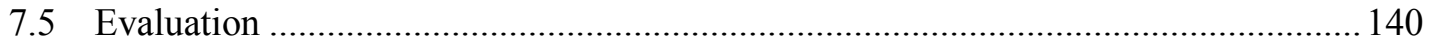

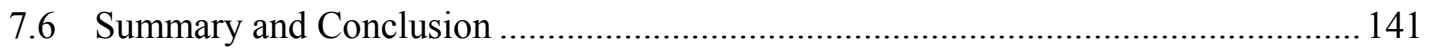

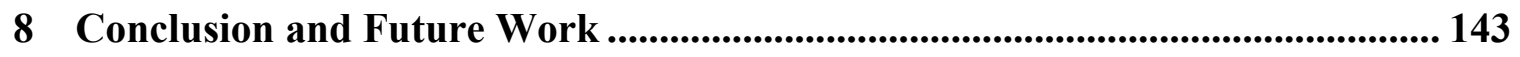

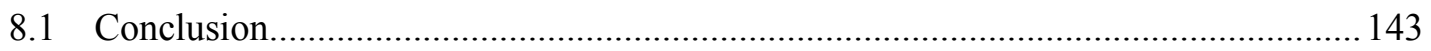

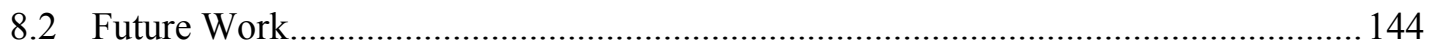

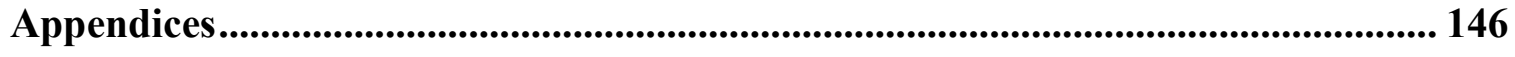

Appendix A: EMD-based Sub-band Adaptive Filtering for Acoustic Echo Cancellation .. 146

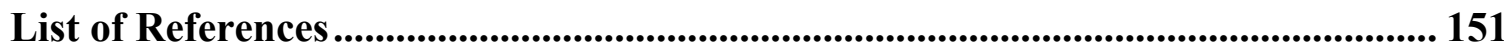




\section{List of Figures}

Figure 1.1: Thesis organization diagram ............................................................. 6

Figure 2.1: Schematic diagram of cardiovascular system ...................................... 11

Figure 2.2: Two cardiac cycles in Wagger's diagram........................................... 13

Figure 2.3: A typical waveform of the arterial blood pressure ................................. 13

Figure 2.4: Riva-Rocci sphygmomanometer used by Korotkoff in his measurements 18

Figure 2.5: Blood pressure measurement using the auscultatory method based on the

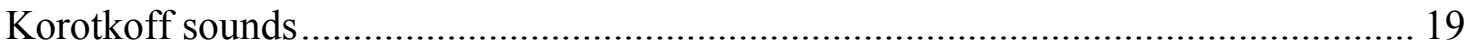

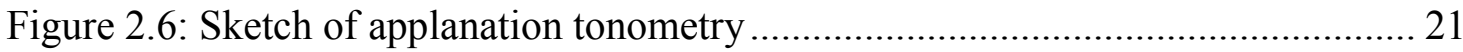

Figure 2.7: The scheme of the volume-clamp method........................................... 22

Figure 2.8: Different shapes of blood pressure waveforms ................................... 24

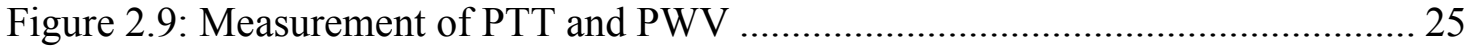

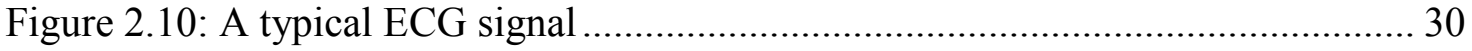

Figure 2.11: Waveform of ECG signal related to the depolarization and repolarization of

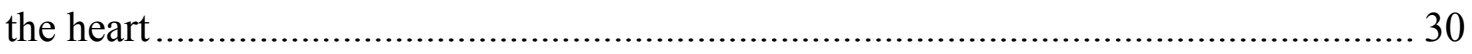


Figure 2.12: Electrodes placement for ECG measurement and the Einthoven's triangle 31

Figure 2.13: Illustration of the reflectance mode and the transmission mode PPG...... 39

Figure 2.14: Peak shifting phenomenon illustrated by the combination of two Gaussian curves

Figure 2.15: Framework of the Eulerian video magnification method 45

Figure 3.1: The scheme of EEMDA algorithm 53

Figure 3.2: The original ECG signal and noise used in this chapter 54

Figure 3.3: IMF distribution of EMD and EEMD for the noisy ECG signal. The noisy ECG signal is contaminated by Gaussian noise with the SNR value of $10 \mathrm{~dB}$. 56

Figure 3.4: The power spectra of corresponding IMFs for the noisy ECG signal with 10 dB Gaussian noise 57

Figure 3.5: Delineation of the QRS complex in the EMD domain 58

Figure 3.6: Delineation of the QRS complex in the EEMD domain 59

Figure 3.7: The boundary lines of the QRS complex. The yellow lines are the boundary lines. The noisy signal is the clean ECG signal contaminated by the contact noise with

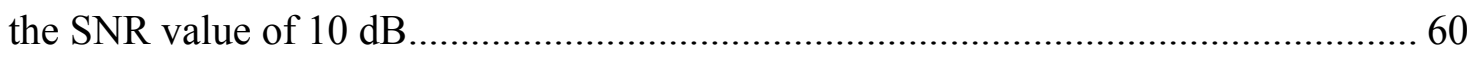

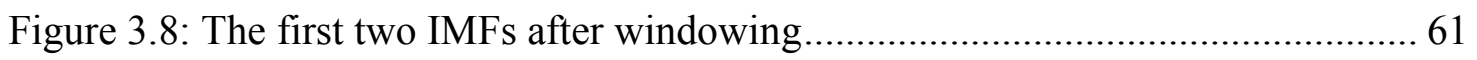


Figure 3.9: The noisy signal, pure Gaussian noise, and the reconstructed signal as the reference input of the adaptive filter 63

Figure 3.10: The noisy signal, pure muscle artifacts, and the reconstructed signal as the reference input of the adaptive filter

Figure 3.11: The noisy signal, pure motion artifacts, and the reconstructed signal as the reference input of the adaptive filter 64

Figure 3.12: The denoising results of the Gaussian noisy signal with SNR value of 10 $\mathrm{dB}$ 65

Figure 3.13: The EEMDA method de-noising results of the contact noisy signal with SNR value of $10 \mathrm{~dB}$ 66

Figure 3.14: The EEMDA method de-noising results of the contact noisy signal....... 68

Figure 3.15: Interface designed by MATLAB GUI 69

Figure 4.1: Characteristic parameters and PTT determined from one set of synchronous ECG and finger PPG signals used for blood pressure estimation 72

Figure 4.2: Ideal PPG signal and its characteristic parameters 73

Figure 4.3: Clinical PPG signal without visible dicrotic notch 74

Figure 4.4: Two-Gaussian-curve model for PPG signal 77

Figure 4.5: Four-Gaussian-curve model for PPG signal 77 
Figure 4.6: Absolute differences between the original PPG signal and the fitted curves by using two-Gaussian-curve modeling and four-Gaussian-curve modeling 78

Figure 4.7: Illustration of the symmetrical curve fitting method 80

Figure 4.8: Second derivative of the original PPG signal and the zero-crossing points 82

Figure 4.9: Secondary peak detection procedure of SCF method 85

Figure 4.10: Secondary peak detection procedure of GCF method 86

Figure 4.11: Secondary peak detection procedure of adaptive curve fitting method.... 87

Figure 4.12: Two Gaussian curves with same standard deviation and amplitude but different mean 88

Figure 4.13: Two Gaussian curves with same standard deviation but different mean and amplitude. 90

Figure 4.14: Two Gaussian curves with different standard deviation, mean and amplitude 91

Figure 4.15: Peak detection thresholds for standard deviation verses the distance between the two Gaussian curves under different amplitude ratios 92

Figure 4.16: Secondary peak detection methods applied on curve composed of two Gaussian curves with the same standard deviation and amplitude but different mean. 94

Figure 4.17: Secondary peak detection methods applied on curve composed of two Gaussian curves with the same standard deviation but different amplitude and mean. 96 
Figure 4.18: Secondary peak detection methods applied on Gaussian curve composed of two Gaussian curves with different standard deviation, amplitude, and mean 98

Figure 4.19: Primary peak location comparison between ACF and the four-Gaussiancurve modeling (cycle 1) 100

Figure 4.20: Primary peak location comparison between ACF and the four-Gaussiancurve modeling (cycle 2)..... 101

Figure 5.1: ABP, ECG, and PPG signal segment extracted from the MIMICII database 106

Figure 5.2: Selected ECG signal and finger PPG signal from MIMIC database. The definition of PTT is determined by the delay between the R-peak and the PPG peak 107

Figure 5.3: Extremes of ABP, R-peak of ECG, primary peaks of PPG, and the calculated PTT 108

Figure 5.4: Scatter plot for the SBP versus PTT and three different types of regression curves 109

Figure 5.5: Scatter plot for PTT versus SBP, DBP, and MBP with cubic regression curve 110

Figure 5.6: Adjusted PPG signal and adjusted PTT by SCF 112

Figure 5.7: Adjusted PPG Signal and adjusted PTT by GCF 113

Figure 5.8: Adjusted PPG Signal and adjusted PTT by ACF 113 
Figure 5.9: R-squared values between the adjusted PTT and the blood pressure by using SCF, GCF, and ACF detection methods 114

Figure 5.10: Scatter plot for SBP versus adjusted PTT and different regression curves 115

Figure 5.11: The Bland-Altman plot for the estimated and the reference blood pressure under different regression methods 116

Figure 5.12: The Bland-Altman plot for the estimated and the actual blood pressure by SCF and ACF methods 117

Figure 5.13: Boxplot of the average ME for the systolic blood pressure estimation by SCF and ACF methods 118

Figure 6.1: Framework of Eulerian video magnification method 123

Figure 6.2: Bound of the magnification factor $\alpha$ 124

Figure 6.3: Two frames of the wrist video sequence. The output frames display the subtle changes on the wrist 125

Figure 6.4: Three-dimensional surface plot of one frame of the input video 126

Figure 6.5: The selected location for wrist pulse signal measurement and its corresponding waveform. 127

Figure 6.6: Finger PPG signal measured by Arduino board and PulseSensor..... 127 
Figure 6.7: Two-Gaussian curve modeling for the wrist pulse signal detected by Eulerian video magnification method

Figure 6.8: Two-Gaussian curve modeling for the PPG pulse signal measured by the Arduino board and the PulseSensor

Figure 7.1: Two frames from one of the original video sequence showing the color changing on the wrist and neck caused by blood pulse. 135

Figure 7.2: Three-dimensional surface plot of one frame 135

Figure 7.3: Selected pulse detection area of interest 137

Figure 7.4: Pulse detection by using Eulerian color magnification on wrist and neck simultaneously.

Figure 7.5: The temporal filter used to amplify the color variation 138

Figure 7.6: The Arduino board and the pulse detecting sensors 140

Figure 7.7: Pulse signals detected from the Arduino board and the PulseSensor ....... 140

Figure 7.8: Comparison of the time difference between the detected pulses on the wrist and the neck through the usage of EVM and the Arduino board with PulseSensor ... 141

Figure 9.1: Structure of NSAF system 147

Figure 9.2: Structure of the EMD-based sub-band adaptive filtering system 148

Figure 9.3: ERLE values of the EEMD sub-band adaptive filtering structure with subband number $\mathrm{N}=2,4,6,8$ 
Figure 9.4: ERLE values of different EMD-based sub-band adaptive filtering system under the exact-modeling and under-modeling scenarios .........................149 


\section{List of Tables}

Table 2.1: Arterial blood pressure classification and thresholds 15

Table 2.2: Grading criteria of AAMI and BHS 16

Table 3.1: The comparison of the de-noised results of the EEMDA method and the EMDbased method for the first 46000 samples of selected records. 67

Table 4.1: Absolute difference between original Gaussian curves and the fitting curves by using different methods 95

Table 4.2: Absolute difference between original Gaussian curves and the fitting curves by using different methods 95

Table 4.3: Absolute difference between original Gaussian curves and the fitting curves by using different methods 97

Table 4.4: Absolute difference between original Gaussian curves and the fitting curves by using different methods

Table 5.1: Different regression models of SBP-PTT on three-patient samples 111

Table 5.2: The correlation between PTT and each type of ABP for three-patient samples 
Table 5.3: Mean error and standard deviation of different secondary wave detection methods for patient \#3302803

Table 6.1: Comparison of the values of different characteristic parameters. 130

Table 7.1: Values of parameters used in Eulerian color magnification to generate the amplified output ... 


\section{List of Appendices}

Appendix A: EMD-based Sub-band Adaptive Filtering for Acoustic Echo Cancellation 


\section{List of Acronyms}

\begin{tabular}{|c|c|}
\hline Acronyms & Definition \\
\hline AAMI & $\begin{array}{l}\text { Association for the Advancement of Medical } \\
\text { Instrumentation }\end{array}$ \\
\hline $\mathrm{ACF}$ & adaptive curve fitting \\
\hline AEC & acoustic echo cancellation \\
\hline ARMAX & $\begin{array}{l}\text { auto-regressive moving average with eXogenous } \\
\text { input }\end{array}$ \\
\hline ARX & auto-regressive with eXogenous input \\
\hline $\mathrm{BIH}$ & Beth Israel Hospital \\
\hline $\mathrm{BJ}$ & box-jenkins \\
\hline $\mathrm{CO}$ & cardiac output \\
\hline DBP & diastolic blood pressure \\
\hline
\end{tabular}




\begin{tabular}{ll} 
ECG & electrocardiogram \\
EEMD & ensemble empirical mode decomposition \\
EMD & empirical mode decomposition \\
EEMDA & EEMD-adaptive \\
EMG & electromyographic \\
ERLE & echo return loss enhancement \\
EVM & Eulerian video magnification \\
GCF & Gaussian curve fitting \\
GUI & graphic user interface \\
HMFs & intrinsic mode functions \\
HOG & histogram of oriented gradients \\
HR & heart rate \\
\hline
\end{tabular}




\begin{tabular}{|c|c|}
\hline LV & left ventricle \\
\hline MAE & mean absolute error \\
\hline MBP & mean blood pressure \\
\hline ME & mean error \\
\hline MIT & Massachusetts Institute of Techonology \\
\hline MSE & mean-squared error \\
\hline NEMD & noise-modulated empirical mode decomposition \\
\hline NMLS & normalized least mean squares \\
\hline $\mathrm{NN}$ & neural networks \\
\hline NSAF & normalized sub-band adaptive filter \\
\hline $\mathrm{OE}$ & output error \\
\hline PAT & pulse arrival time \\
\hline PPG & photoplethysmograph \\
\hline PSD & power spectral density \\
\hline TT & pulse transit time \\
\hline
\end{tabular}




$\begin{array}{ll}\text { PWV } & \text { pulse wave velocity } \\ \text { RAS } & \text { relative amplitude of secondary peak } \\ \text { RAt } & \text { right atrium } \\ \text { RV } & \text { right ventricle } \\ \mathrm{SaO}_{2} & \text { blood oxygen saturation } \\ & \\ \mathrm{SBP} & \text { systolic blood pressure } \\ \mathrm{SCF} & \text { symmetrical curve fitting } \\ & \\ \mathrm{SDE} & \text { standard deviation of error } \\ \mathrm{SER} & \text { signal-error ratio } \\ \mathrm{SNR} & \text { signal-to-noise ratio }\end{array}$




\section{List of Symbols}

\begin{tabular}{ll} 
Symbols & Definition \\
\hline$\alpha$ & magnification factor of EVM \\
$A$ & curve amplitude \\
$A 1 / A 2$ & amplitude ratio \\
$\beta$ & window shape determinant \\
$c_{i}(t)$ & intrinsic mode function \\
$D$ & length of an arterial segment \\
$e_{\text {lower }}(t)$ & lower envelope \\
$e_{\text {upper }}(t)$ & upper envelope \\
$E_{\text {inc }}$ & incremental elastic modulus \\
$g_{0}$ & \\
&
\end{tabular}


$G_{i} \quad$ adjacent low-pass images of Gaussian pyramid

$h \quad$ thickness of the arterial wall

$h_{i}(t) \quad$ proto-IMF

$H \quad$ number of IMFs contributes to high-frequency noise

I intensity of the transmitted light through the tissue

$I_{0} \quad 0^{\text {th }}$-order modified Bessel function

$I_{b} \quad$ intensity of an injected light beam

$k_{1} \quad$ coefficient of $n_{m a}$

$k_{2} \quad$ coefficient of $n_{e m}$

$l_{n} \quad$ optical path length

$L \quad$ number of IMFs contributes to low-frequency noise

$L_{i}(P) \quad$ Laplacian pyramid

$m_{i}(t) \quad$ mean of the two envelope

M window length

$n_{e m} \quad$ electrode contact noise 


\begin{tabular}{|c|c|}
\hline$n_{m a}$ & muscle artifact \\
\hline$N$ & signal length \\
\hline $\mathbf{N}_{r k}$ & reference input vector \\
\hline$P$ & Cauchy principle value \\
\hline$r$ & linear regression correlation coefficient \\
\hline$r_{i}(t)$ & residual \\
\hline$r_{l p}$ & radius of the spatial low-pass filter \\
\hline$r_{v}$ & vessel radius \\
\hline$R^{2}$ & Square of Pearson correlation \\
\hline$S(r)$ & signal power over spatial frequencies \\
\hline$\tau 1 / \tau 2$ & phase shift ratio \\
\hline$\omega$ & frequency \\
\hline$\omega_{n}$ & light absorption coefficient for each optical segment \\
\hline$w 1 / w 2$ & curve width ratio \\
\hline
\end{tabular}




\begin{tabular}{ll}
$Y(t)$ & Hilbert transform \\
$\theta$ & step size \\
$\rho$ & blood density \\
$\sigma$ & standard deviation \\
$\delta$ & video motion step \\
$\lambda$ & spatial wavelength \\
$\mu$ & \\
\hline
\end{tabular}




\section{Chapter 1}

\section{Introduction}

\subsection{Motivation}

In recent decades, cardiovascular diseases have become one of the leading causes of death in developed countries. The risk of having arteriosclerosis and hypertension is no longer just for seniors. The age of patients with various cardiovascular diseases has been dropping and the number of people who need daily cardiovascular monitoring has been increasing significantly [1]. At the same time, individual-based health care monitoring has become more and more common because of the intense usage of hospital resources, [2]. Patients also want to be as independent as possible as they age. Therefore, the need for selfmonitoring devices dramatically increases, and the monitoring of cardiovascular parameters in a noninvasive and continuous system is of great interest to both physicians and patients.

One of the most important parameters for the assessment of human cardiovascular system is the arterial blood pressure [3][4][5][6]. High blood pressure, namely hypertension, is regarded as one of the major risk factors that causes cardiovascular 
diseases. Blood pressure variability has been demonstrated to have the ability to predict hypertension [7]. Therefore, continuous blood pressure measurement is extremely important for hypertension prevention, detection, and treatment. As we know, the most precise way to obtain arterial blood pressure is through invasive measurement, in which a catheter is inserted into the patients' vessel [8]. However, this method of measurement demands a high-level operating skill on the part of the doctor or nurse, not to mention the patient may not feeling comfortable with the device and the pain and the potential risk to the patient.

Nowadays, non-intrusive and noninvasive detection methods are becoming the basic requirements for medical devices, especially when the devices are to be used by patients for self-monitoring without the assistance of health care providers [9][10]. At present, the conventional method that is used in clinical and hospital situations to measure the arterial blood pressure is the usage of the bladder and the brachial cuff. This measurement is considered to be the most accurate estimation of arterial blood pressure [11][12]. However, this measurement cannot continuously measure the systolic and diastolic blood pressure at intervals. The importance of continuously tracking the arterial blood pressure during their daily activity exists in the fact that short-term changes in blood pressure can provide previously, undetected necessary information about the patient's health status. Additionally, the discomfort caused by the inflation and deflation of the brachial cuff may lead to alterations of the arterial blood pressure. This cuff-based measurement is especially not suitable when the patients are asleep.

Instead of using the conventional cuff, there have been several studies on measuring the blood pressure noninvasively and continuously, such as the arterial tonometry, the 
column clamp method, and the pulse transit time (PTT). For arterial tonometry, an array of pressure sensors was pressed against the skin over an artery [13]. With the volume-clamp method, the finger arterial pressure was measured by a finger cuff and an inflatable bladder combined with an infrared plethysmogram, which consisted of an infrared light detector and light source [14]. It has also been demonstrated that blood pressure can be obtained by measuring the pulse wave velocity (PWV) [15]. However, the measurement of PWV is a very challenging task since it needs different models, mathematical calculations, accurate measurement of blood flows, and a thorough analysis. Fortunately, PTT has been proven to be a very useful tool to describe the relationship between PWV and the arterial blood pressure [16][17].

Generally, PTT refers to the time that a pulse wave takes to travel between two arterial locations. There have been a number of techniques designed to measure PTT, such as the Ultrasound Doppler and the arterial tonometry [18][19]. The simplest and most convenient way to measure PTT is to calculate the time delay between the R-peak in the electrocardiogram (ECG) signal and the beginning of the following photoplethysmography (PPG) signal [20].

\subsection{Problem Statement}

The correlation between the blood pressure and the PTT has been studied in quite a few research papers [21][22][23]. The delay caused by some of the existing medical devices causes some uncertainties in measuring the PTT [24]. Linear regression was used to estimate the blood pressure, which led to an estimation error of up to $25 \mathrm{mmHg}$ [25]. A parameter extracted from the dicrotic notch of the PPG signal, called relative amplitude of 
secondary peak (RAS), was used for the estimation of systolic blood pressure in [26]. The result showed that the underestimated bias could be largely reduced by introducing RAS.

There are also some other problems for the existing PTT measurement methods. To obtain the PTT, previous knowledge of the ECG signal and the PPG signal is required. In addition, the measured ECG signal is often contaminated with severe noise from different sources. The dicrotic notch of the PPG signal usually cannot be easily observed. To overcome the problems listed above and to obtain an accurate measurement of the PTT, new technologies and algorithms need to be investigated.

\subsection{Thesis Objectives and Summary of the Results}

The major objective of this thesis is to develop new algorithms that are able to accurately measure PTT. One of the frequently-used methods is to calculate the time difference between certain characteristic parameters of the ECG signal and the PPG signal in one

pulse cycle. For the ECG signal, a novel signal enhancement method based on the Empirical Mode Decomposition (EMD) algorithm and the adaptive filtering technique was introduced to remove different types of noise from the corrupted ECG signal. The proposed algorithm has been proved to be able to remove both Gaussian noise and the contact noise contained in the noisy ECG signal.

For the PPG signal, the objective is to extract the embedded characteristic parameters of the PPG signal. Gaussian curves were employed to model the PPG signal and three primary peak detection methods were developed. The correlation between the adjusted PTT and the arterial blood pressure was evaluated. . The simulation results verified that the PTT obtained by detecting the delay between the R-peak of the ECG signal and the primary 
peak of the following PPG signal can determine the arterial blood pressure continuously and noninvasively.

A recently developed algorithm, called the Eulerian video magnification, was also introduced to noninvasively measure the wrist pulse signal and the PTT. The experiments demonstrated that the PTT can be obtained by extracting the color changes on different locations on the human skin and locating the peaks of the observed signal.

\subsection{Outline of the Thesis}

This thesis consists of eight chapters. The structure of this thesis is shown in Figure 1.1.

Chapter 1 is the introduction, which briefly describes the motivation of the study, the statement of the problems, and the objectives of the thesis. This chapter also presents the outline of the research and summarizes the main contributions.

Chapter 2 provides the background information related to the research conducted in this thesis. The physiology basis of human cardiovascular system and the history of the blood pressure measurement is reviewed, and the recent development and limitations of the PTT measurement are depicted. In particular, this chapter reviews the basic concepts of the ECG and PPG signals. The database of the signals and the technologies on enhancing the signals are introduced.

As one of the most important parameters in measuring the PTT, the enhancement of the ECG signal is discussed Chapter 3. The EMD-based algorithm and the adaptive filtering technique are explained in detail. 


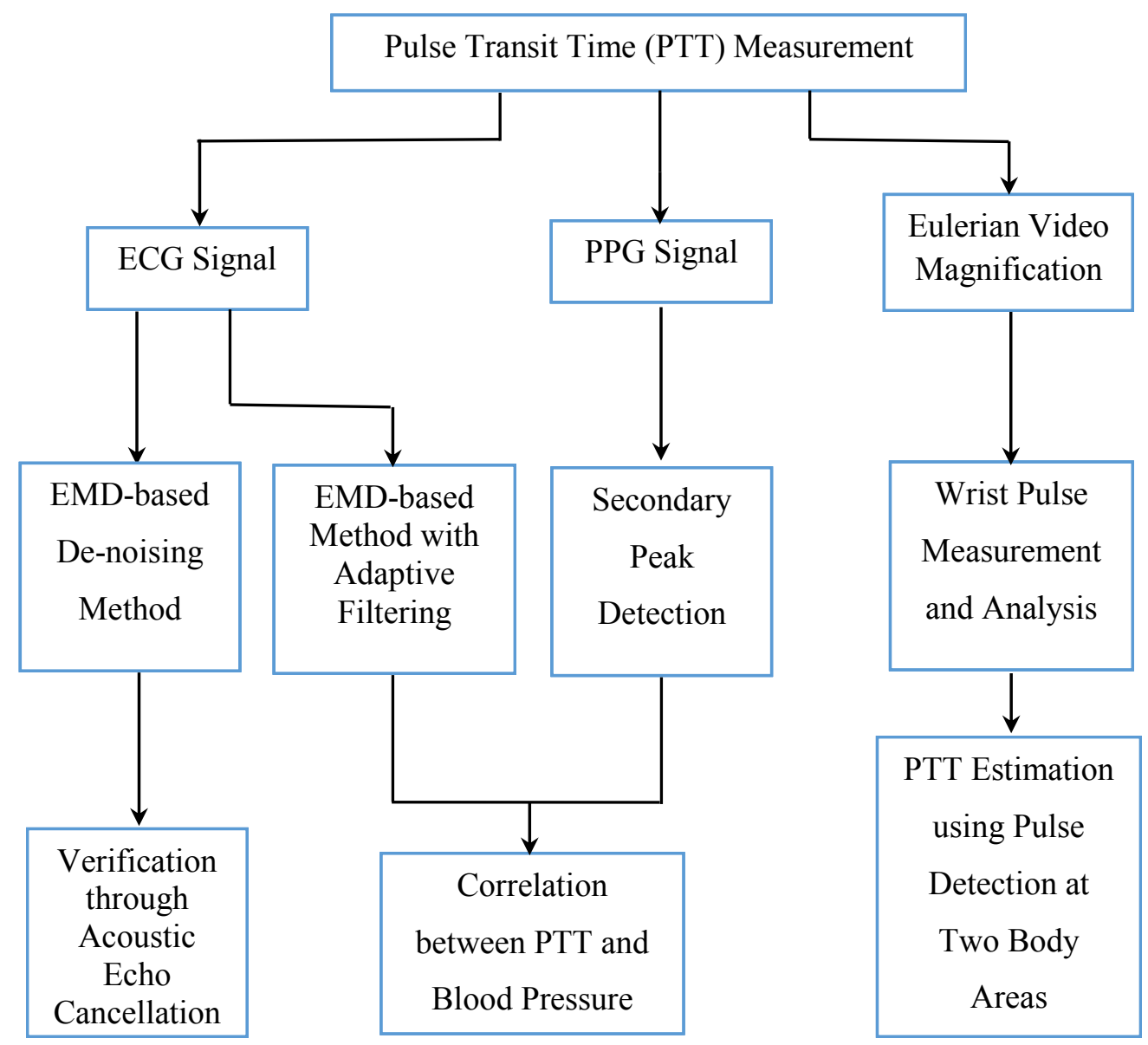

Figure 1.1: Thesis organization diagram

Chapter 4 presents the processing of the PPG signal, which is another important component of the PTT measurement. Several methods are introduced to extract the characteristic parameters of the PPG signal.

After the enhanced ECG and PPG signals are acquired, it is shown how the adjusted PTT is calculated in Chapter 5. The correlation between the adjusted PTT and the arterial blood pressure is compared with the correlation between the PTT (which is obtained from 
the original ECG and PPG signals from the MIMIC database) and the arterial blood pressure.

In Chapter 6, a recently proposed method called Eulerian video magnification framework is introduced to measure and analyze the wrist pulse signal. Location "guan", which was used in the traditional palpation technique, is selected for the experiment. The two-Gaussian curve modeling method is employed for fitting the wrist pulse signal.

Chapter 7 introduces the Eulerian video magnification framework to measure the PTT through detecting the blood flow under the human skin. The performance of the described framework is verified by comparing it with the conventional techniques for PTT measurement.

Chapter 8 concludes the thesis and provides some suggestions on possible future work.

\subsection{Statement of Originality and Main Contributions}

This thesis results in a number of publications in the field of signal enhancement and pulse transit time measurement. I certify that the results presented in this thesis are original work from me and my supervisors. I am the primary author and my supervisors, Professor Rafik A. Goubran and Professor X. Liu, are the co-authors in recognition of their technical contributions. I acknowledge their helpful guidance and support.

The main contributions of this thesis include:

1. The development of a novel ECG signal enhancement method based on EEMD and the adaptive filtering techniques. This contribution resulted in the following publications: 
[27] X. He, R. A. Goubran, X. P. Liu, "Signal enhancement of wearable ECG monitoring sensors based on ensemble empirical mode decomposition," in Proc. of the IEEE International Symposium on Medical Measurements and Applications (MeMeA), Bari, May 2011, pp. 433-436.

[28] X. He, R. A. Goubran, X. P. Liu, "Ensemble empirical mode decomposition and adaptive filtering for ECG signal enhancement," in Proc. of the IEEE International Symposium on Medical Measurements and Applications (MeMeA), Budapest, May 2012, pp. 1-5.

2. The development of an EMD-based algorithm for acoustic echo cancellation and the design of a new EMD-based sub-band adaptive filtering structure. This contribution resulted in one journal publication:

[29] X. He, R. A. Goubran, X. P. Liu, "EMD-based sub-band adaptive filtering for acoustic echo cancellation," International Journal of Speech Technology, vol. 17, pp. 37-42, March 2014.

3. The development of a cuff-less non-intrusive approach to measure the arterial blood pressure that is suitable for continuous measurement. This contribution resulted in the following publication:

[30] X. He, R. A. Goubran, X. P. Liu, "Evaluation of the correlation between blood pressure and PTT," in Proc. of the IEEE International Symposium on Medical Measurements and Applications (MeMeA), Gatineau, QC, May 2013, pp. 17-20. 
4. The design of three secondary peak detection methods to improve the correlation between the blood pressure and the PTT, and to remove the dicrotic notch impact on the PPG signal. This contribution resulted in one journal publication:

[31] X. He, R. A. Goubran, X. P. Liu, "Secondary peak detection of PPG signal for continuous cuff-less arterial blood pressure measurement," IEEE Transaction on Instrumentation and Measurement, vol. 63, pp. 1431-1439, June 2014.

5. The measurement and modeling of the wrist pulse signal. The Eulerian video magnification framework is first applied to noninvasively detect the wrist pulse and the two-Gaussian curve modeling method is used to analyze the wrist pulse signal. This contribution resulted in the following paper:

[32] X. He, R. A. Goubran, X. P. Liu, "Wrist pulse measurement and analysis using Eulerian video magnification," in Proc. of the IEEE International Conference on Biomedical and Health Informatics, Las Vegas, February 2016.

6. The development of a non-intrusive way to observe the blood flow and obtain the PTT. The performance and practicability of the proposed Eulerian video magnification framework were also evaluated. This contribution resulted in a conference publication:

[33] X. He, R. A. Goubran, X. P. Liu, "Using Eulerian video magnification framework to measure pulse transit time," in Proc. of the IEEE International Symposium on Medical Measurements and Applications (MeMeA), Lisbon, June 2014, pp. 50-53. 


\section{Chapter 2}

\section{Background}

In this chapter, the background of this thesis is introduced. Section 2.1 provides basic information on human cardiovascular system and the arterial blood pressure. Section 2.2 lists the state-of-the-art, noninvasive arterial blood pressure measurement techniques. Section 2.3 describes one of the most commonly used methods to estimate the arterial blood pressure: pulse transit time (PTT), and in particular, the definition of PTT used in this thesis. Section 2.4 provides a review on the techniques used to enhance the ECG signals, and Section 2.5 focuses on the measurement of PPG signals. Section 2.6 provides the theory and concept of the Eulerian video magnification method.

\subsection{Cardiovascular System}

The cardiovascular system of a human being consists of the heart and the blood vessels. There are three primary functions of human cardiovascular system [34]. The first is to transport oxygen, nutrients, and fluid to organs and tissue. The second is to generate and distribute hormones. The third is to regulate the body temperature. Figure 2.1 is a schematic diagram of human cardiovascular system, showing the pulmonary circuit and the systemic 
circuit [35]. In the pulmonary circuit, the oxygen-poor blood leaves the heart through the right ventricle (RV) and travels through the lungs where carbon dioxide is released. Then the oxygen-rich blood is recalled by the left atrium (LAt). In the systemic circuit, the oxygen-rich blood is distributed from the left ventricle (LV) through the body and then the oxygen-poor blood is recalled to the right atrium (RAt).

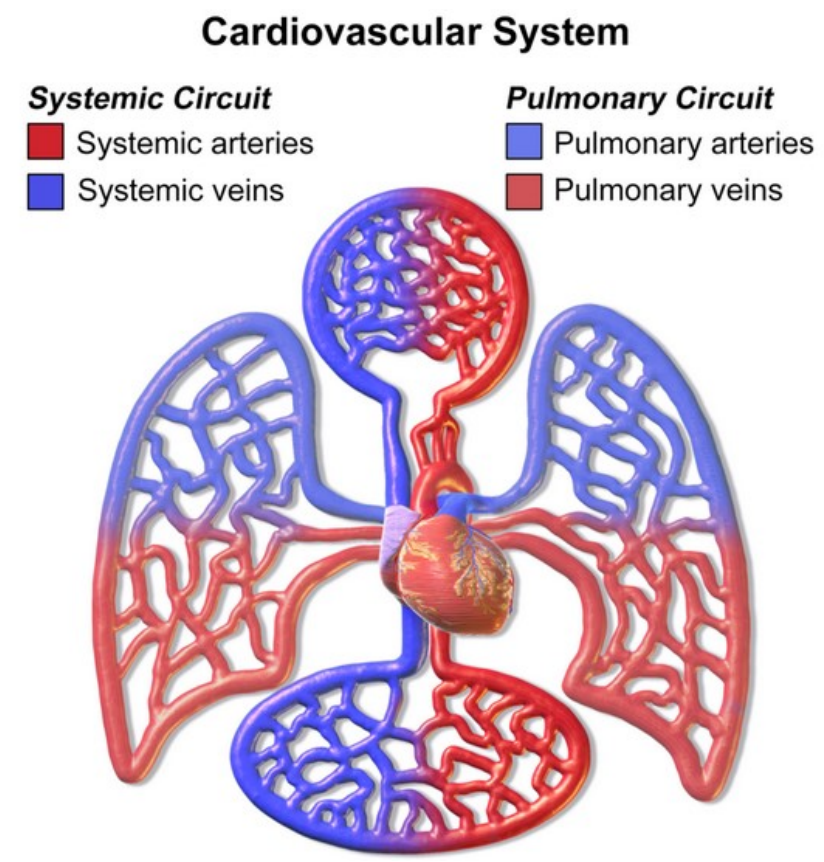

Figure 2.1: Schematic diagram of cardiovascular system [35]

The blood flow through the pulmonary circuit and the systemic circuit is coordinated by the cardiac cycle. Generally speaking, the cardiac cycle includes the diastolic, the systolic, and the intervening pause and involves five steps [36]:

1) Diastole: the blood passively flows from the two atria into the two ventricles. The semi-lunar aorta and pulmonary valves remain closed. The valves between the atria and the ventricles (A-V valves) open. 
2) Atrial systole: all the blood in the atria flows into the ventricles as the atria contract.

3) Isovolumic contraction: the $\mathrm{A}-\mathrm{V}$ valves close and the ventricles start contracting to pump the blood into the aorta and pulmonary arteries.

4) Ventricular ejection: the ventricles keep on contracting until close to empty and the semi-lunar aorta and pulmonary valves are open.

5) Isovolumic relaxation: the ventricles begin to relax and pressure decreases. The semi-lunar valves close again.

Figure 2.2 is the Wigger's diagram showing two cardiac cycle events occurring in the left ventricle [37].

The pressure exerted by the blood upon the blood vessel walls is defined as the arterial blood pressure, which is controlled by human cardiovascular system. The arterial blood pressure increases and decreases within each cardiac cycle. Nowadays, blood pressure measurement has become a routine inspection item for any medical examination, along with body temperature, respiratory rate, and pulse rate. It varies depending on the age, gender, activity, and the health statues of the subjects. Usually, the arterial blood pressure refers to the pressure in the systemic circuit and is expressed in terms of systolic blood pressure (SBP) over diastolic blood pressure (DBP). The SBP is the maximum pressure. It occurs when the blood is pumped from the left ventricle into the aorta. The DBP is the minimum pressure. It occurs when the blood flows from the atria to the ventricles. Normal SBP falls in the range of 90 to $120 \mathrm{mmHg}$ and the DBP lies between 60 and $80 \mathrm{mmHg}$. Figure 2.3 shows a typical waveform of normal arterial blood pressure. The red curve represents the aortic pressure. The definitions of SBP, DBP, and mean blood 
pressure (MBP) are illustrated in [38]. MBP is usually calculated using the following equation:

$$
M B P=\frac{1}{3} \times(S B P+2 \times D B P)
$$

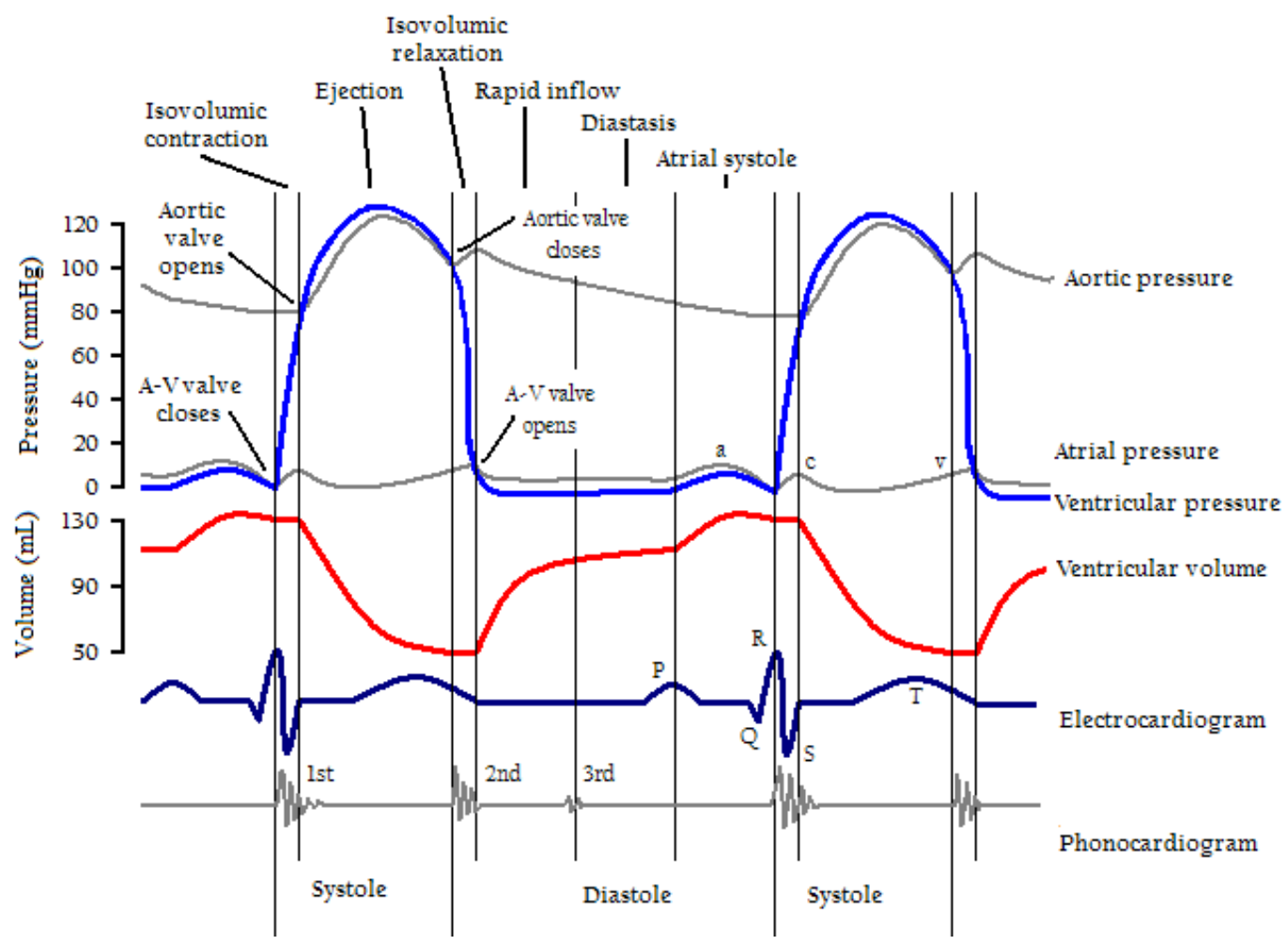

Figure 2.2: Two cardiac cycles in Wagger's diagram [37]

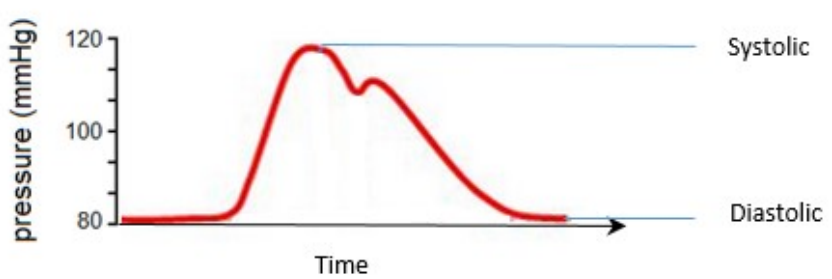

Figure 2.3: A typical waveform of the arterial blood pressure 
If the arterial blood pressure is lower than the normal value, it is called hypotension; and if the pressure is consistently higher than the normal value, it is called hypertension. Approximately $26 \%$ of the world's population is affected by hypertension [39]. Long term hypertension can lead to kidney failure, heart disease, and stroke. Heart attack or stroke caused by hypertension is considered to be one of the major risk factors of cardiovascular disease, which is the most common cause of death in Western countries [40]. Long term hypertension is often undetectable due to infrequent monitoring and the absence of symptoms. To depict the classification of the arterial blood pressure levels, Table 2.1 presents different blood pressure categories and value thresholds adopted by the American Heart Association for adults aged 18 years and older [41][42].

The arterial blood pressure thresholds in Table 2.1 are obtained from clinical measurements during medical examinations. While blood pressure is fluctuating all the time, the highest readings are often obtained in the afternoons and the lowest readings at night [43]. Previous clinical research proves that compared to day-time blood pressure, night-time blood pressure is a stronger indicator of cardiovascular events [44]. For that reason, the blood pressure values collected from medical examinations can only reveal a partial view of the nature of hypertension. To achieve a full view of the arterial blood pressure, 24-hour continuous measurement of the blood pressure is needed. 
Table 2.1: Arterial blood pressure classification and thresholds [41][42]

\begin{tabular}{ccc}
\hline Category & SBP(mmHg) & DBP(mmHg) \\
\hline Hypotension & $<90$ & $<60$ \\
Desired & $90-119$ & $60-79$ \\
Prehypertension & $120-139$ & $80-89$ \\
Stage 1 hypertension & $140-159$ & $90-99$ \\
Stage 2 hypertension & $160-179$ & $100-109$ \\
Hypertension emergency & $\geq 180$ & $\geq 110$ \\
Isolated systolic hypertension & $\geq 140$ & $<90$ \\
\hline
\end{tabular}

Ambulatory blood pressure measurement was developed to measure the blood pressure during the subject's daily activities. The most common way for the ambulatory blood pressure measurement to be taken is through the usage of brachial oscillometric blood pressure cuffs to obtain the blood pressure value every twenty to thirty minutes throughout the day and night. However, there are limitations on the ambulatory measurements. In particular, it is a discontinuous measurement due to its intermittent nature. Another limitation is that the oscillometric devices would bring discomfort or anxiety to the subjects, especially while they are sleeping. Therefore, new techniques for 24-hour cuff-less, noninvasive, continuous monitoring of the subject's blood pressure variation are in demand. 


\subsection{Blood Pressure Measurement Techniques}

\subsubsection{Validation Standards for Blood Pressure Measurement}

In the 1960 s and 1970 s, protocols used to validate the blood pressure measuring system were proposed. Two of the most widely used standard protocols for blood pressure measurement validation are the standard set by the US Association for the Advancement of Medical Instrumentation (AAMI) published in 1986 and the British Hypertension Society (BHS) published in $1990[65,66]$. Both of them set a tolerated error range when comparing the estimated blood pressure measurements by a given technique. Table 2.2 shows the grading criteria of the two validation standards.

Table 2.2: Grading criteria of AAMI and BHS

\begin{tabular}{|c|c|c|c|}
\hline \multirow[t]{3}{*}{ AAMI } & \multicolumn{3}{|c|}{ Statistical comparison } \\
\hline & Mean difference & \multicolumn{2}{|c|}{ Mean Standard deviation } \\
\hline & $<5 \mathrm{mmHg}$ & \multicolumn{2}{|c|}{$<8 \mathrm{mmHg}$} \\
\hline \multirow[t]{2}{*}{ BHS } & \multicolumn{3}{|c|}{ Absolute difference between standard and test technique } \\
\hline & $\leq 5 \mathrm{mmHg}$ & $\leq 10 \mathrm{mmHg}$ & $\leq 15 \mathrm{mmHg}$ \\
\hline Grade A & $60 \%$ & $85 \%$ & $95 \%$ \\
\hline Grade B & $50 \%$ & $75 \%$ & $90 \%$ \\
\hline Grade C & $40 \%$ & $65 \%$ & $85 \%$ \\
\hline Grade D & \multicolumn{3}{|c|}{ Worse than Grade C } \\
\hline
\end{tabular}


All the percentages need to be greater than or equal to the gold standard measurements obtained from the mercury sphygmomanometer for a certain grade for both SBP and DBP.

\subsubsection{Direct Measurement}

The most accurate way to measure the arterial blood pressure is the direct measurement through an arterial line. A cannula needle is placed in an artery and is connected to a sterile fluid-filled system [45]. Although the measured arterial blood pressure is constantly monitored and relatively accurate, this technique is only applied in human and veterinary intensive care medicine or for research purposes because inserting the cannula is painful and is usually done under anesthesia. Besides that, the cannulation for invasive arterial blood pressure measurement is occasionally associated with complications like thrombosis, infection, and bleeding [46]. Due to the high potential danger of invasive arterial monitoring, close supervision on the patients are required. This method is not suitable for daily monitoring. To avoid these drawbacks, techniques that noninvasively measure the arterial blood pressure were developed.

\subsubsection{Occlusive Measurement}

Noninvasive blood pressure measurements are quicker and safer than invasive measurements. They are less unpleasant and less painful to the subjects. Since the $19^{\text {th }}$ century, noninvasive blood pressure measurement has been the objective of clinical test and technological developments [47]. Jules Herisson developed a mercury based device to measure the pulse pressure in 1835 [48]. Poseuille developed the first hemodynameter to monitor the blood pressure continuously [49]. Vierordt developed a pioneer pulse recorder to record the beating of a radial artery pulse which was published in a paper in 1855 [50]. 
At the end of the $19^{\text {th }}$ century, Scipione Riva-Rocci developed the first sphygmomanometer with an inflation cuff, shown in Figure 2.4 [51]. By occluding the whole upper arm with a cuff, there was no more need for locating specific arteries. SBP could be measured by using a mercury sphygmomanometer along with palpation. The popularization of the modern sphygmomanometers based on inflation brachia cuffs was done by Harvey Cushing [50].

In 1905, Nikolai Korotkoff established the basics of the modern auscultation blood pressure measurement by characterizing the sounds generated by the blood flow [51]. The discovered blood flow sounds were also known as "Korotkoff sounds". Now both SBP and DBP were able to be measured, using the upper-arm cuff technique developed by RivaRocci. The blood pressure measurement using the auscultatory method based on the Korotkoff sounds is illustrated in Figure 2.5 [52]. The grey line represents the cuff pressure and the red curve represents the arterial blood pressure.

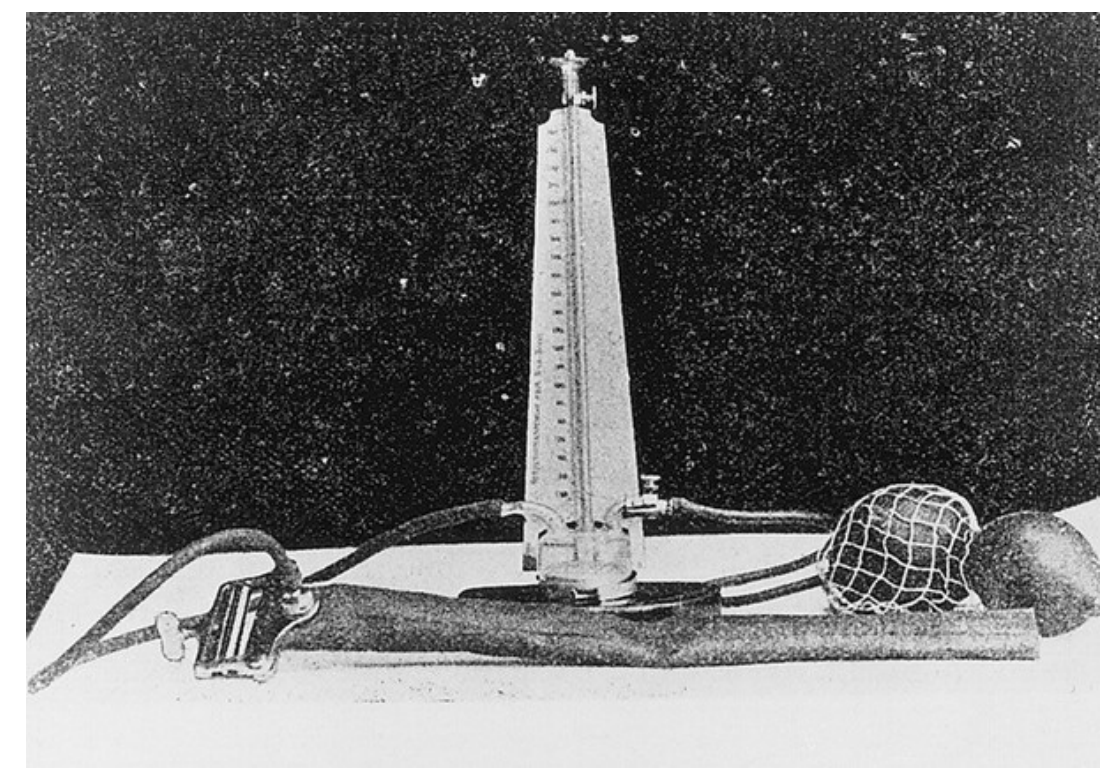

Figure 2.4: Riva-Rocci sphygmomanometer used by Korotkoff in his measurements [51] 
As shown in Figure 2.5, the procedure of the auscultatory method, based on the Korotkoff sounds, can be described as follows [53]:

1. Place the brachial cuff around the whole upper-arm to occlude the brachial artery. Then place a stethoscope below the cuff over the artery.

2. Inflate the cuff to a pressure above $150 \mathrm{mmHg}$. The brachial artery is completely occluded when the cuff pressure is higher than the SBP and there is no blood flow and no sound.

3. Deflate the cuff slowly until a first sound is heard through the stethoscope. Cuff pressure is associated with the SBP, as shown in Figure 2.5.

4. Deflate the cuff gradually until no more sounds can be heard through the stethoscope. At this time, the artery is fully open and the blood flows freely. Cuff pressure corresponds to the DBP, as shown in Figure 2.5.
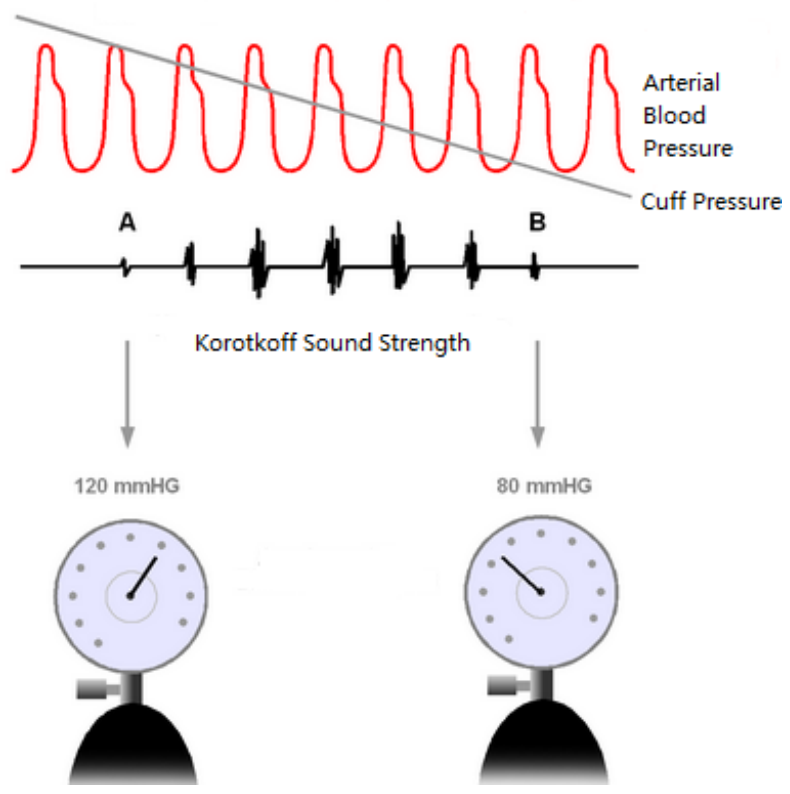

Figure 2.5: Blood pressure measurement using the auscultatory method based on the Korotkoff sounds [52] 
In the auscultatory method, the brachial cuff, being placed around the upper-arm at roughly the same vertical height as the heart, is attached to a mercury sphygmomanometer, an aneroid sphygmomanometer, or a hybrid sphygmomanometer. Nowadays, the mercury manometer which measures the height of a column of mercury is regarded as the gold standard for arterial blood pressure measurement [53].

Based on the procedure of the auscultatory method, it is obvious that the auscultatory measurements provide a point-measurement only. However, frequent measurements will lead to soreness in the subject's arm. Also, it requires the intervention of an operator to inflate and deflate the brachial cuff and interpret the Korofkoff sounds. For these reasons, this method is relatively subject to human errors. The deflation rate and the size of the brachial cuff also affects the accuracy of the measurement [47].

The oscillometric method was developed to overcome the limitation of the operatordependent nature of the auscultatory method. Oscillometric devices were first utilized in 1876 [54]. An oscillometric device employs a sphygmomanometer cuff with an electronic pressure sensor to detect cuff pressure oscillations caused by the oscillations of the blood flow. The inflation and the deflation of the cuff is automatic. Thus oscillometric measurement may be suitable for automated home-monitoring. Over the deflation period of the oscillometric method, the extracted oscillometric pulses are known as the oscillometric waveform. The maximum amplitude of the oscillometric waveform is reached when the pressure approximates the MBP. An accurate SBP and DBP cannot directly be provided from the oscillometric method. Nevertheless, the oscillometric method raised the standard of unsupervised measurement of blood pressure and started a new way of cardiovascular monitoring [55]. 


\subsubsection{Semi-Occlusive Measurement}

During the $20^{\text {th }}$ century, some semi-occlusive methods were developed after the auscultatory and oscillometric methods. They are called semi-occlusive because in these methods, the blood flow is never completely impeded. Applanation tonometry was introduce by Pressman and Newgard in 1963 [56]. Figure 2.6 is a sketch of the arterial blood pressure measurement using applanation tonometry. The tonometer is about the size of a pen. The basic principle of the method is that the vertical displacements detected by the tonometer are proportional to the arterial blood pressure [57][58]. Therefore, no absolute blood pressure can be obtained. The Vasotrac device created a way to get an absolute estimation of MBP. This was achieved by gently compressing and decompressing the radial artery for twelve to fifteen beats. As the tonometer pressure reached the maximum signal amplitude, the absolute estimation of MBP was obtained [59].

Applanation tonometry is regarded as the gold standard method to noninvasively assess the arterial blood pressure waveforms in some reseach. Several commercial medical devices have been invented and have been introduced for clinical use, such as the supervised SphygmoCor, and the unsupervised Vasotrac [60].

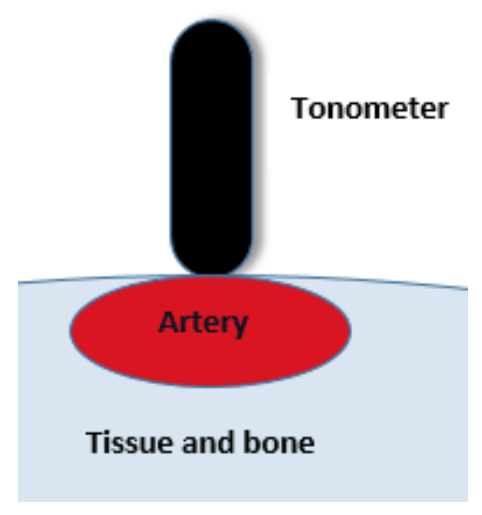

Figure 2.6: Sketch of applanation tonometry 
In 1969, Czech physiologist Penaz introduced the volume-clamp measurement, which employed a finger cuff and an inflatable bladder with a photoplethysmographic (PPG) device [61]. A feedback loop and a servo control system was used to clamp the volume of the blood vessels in the finger which was placed in the sensor. Figure 2.7 shows the scheme of the volume-clamp measurement.

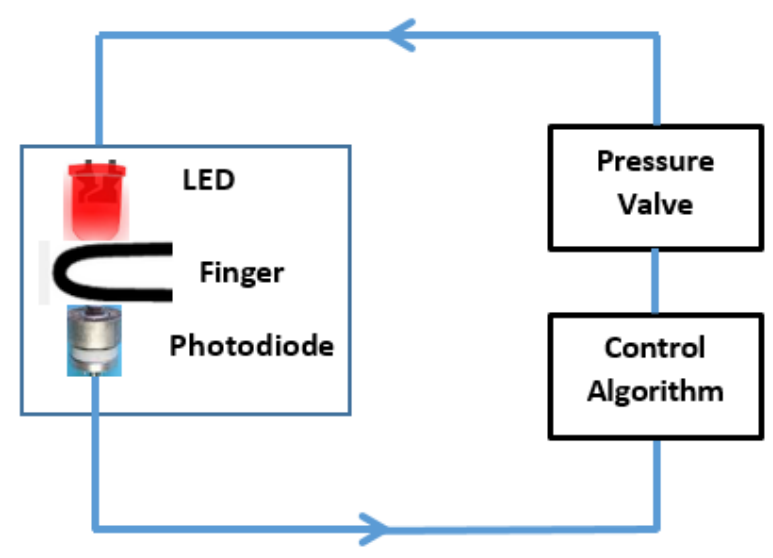

Figure 2.7: The scheme of the volume-clamp method

In the volume-clamp method, the proper unloaded diameter of the arteries, at which the finger cuff pressure is equal to the intra-arterial pressure and the transmural pressure across the arterial walls is zero, needs to first be determined at first. The servo control system defines a set point, which is the signal of the PPG device being clamped. The light detector provides the measured value. Once the volume of the blood vessels is fixed, the changes in the arterial blood pressure can be determined by the changes in the external pressure needed to keep the volume constant.

With the development of the volume-clamp method, 24-hour blood pressure variations could be recorded. The cardiovascular parameters during the subjects' daily activities could be acquired and analyzed. One of the limitations of the volume-clamp 
method is that since it is a semi-occlusive method, continuous long-term measurement may lead to periods of venous congestion [61].

\subsubsection{Pulse Wave Velocity}

The use of pulse wave velocity (PWV) as a measurement in estimating the arterial blood pressure has been studied since the 1960s. An innovative family of noninvasive arterial blood pressure measurement techniques based on the PWV principle was developed in the 1990s. The primary advantage of these techniques is that they can measure PWV values continuously and non-occlusively. After a calibration procedure, PWV values are then translated into arterial blood pressure values. PWV has been recognized as integral to the diagnosis and treatment of hypertension by the European Society of Hypertension [62].

It is believed that the velocity of the blood flow depends on the arterial blood pressure [63]. PWV is defined as the velocity of an arterial pressure pulse traveling along the arterial tree. The relationship between PWV and the incremental elastic modulus of the arterial wall can be described by the Moens-Korteweg equation [64].

$$
P W V=\sqrt{E_{\text {inc }} \cdot h /\left(2 r_{v} \rho\right)}
$$

where $E_{\text {inc }}$ is the incremental elastic modulus, $h$ is the thickness of the arterial wall, $r_{v}$ is the vessel radius, and $\rho$ is the blood density. So for constant wall thickness, vessel ratio, and blood density, PWV is proportional to the square root of the incremental elastic modulus or its distensibility. The Moens-Korteweg equation relies on some necessary assumptions or simplifications. One of these is that the changes in vessel area and in the wall thickness are insignificant. Hence, PWV can be considered carrying information on the arterial stiffness for large elastic arteries, where the wall thickness to vessel radius ratio is relatively fixed. 
Basically, there are four major determinants of PWV, namely age, gender, arterial blood pressure, and heart rate. With increasing age, the arterial stiffness increases [67]. With regard to gender, there is no difference in PWV for children. Young and middle-aged healthy women have lower PWV than healthy men. After age 50, there is no significant difference in PWV between women and men [68]. PWV is proportional to the arterial blood pressure. With increased blood pressure, the blood flow propagates faster along the vessels [69]. Also, increasing heart rate is associated with increasing PWV [70].

PWV has been regarded as the gold standard in assessing the arterial stiffness and has been used as a surrogate for the arterial blood pressure during the last two decades. PWV is also a robust parameter in predicting cardiovascular events [69]. The stiffness of the artery increases when blood flows from the aortic to the periphery. Because of this increase in arterial stiffness, the shapes of aortic and brachial blood pressure waveforms are different, as shown in Figure 2.8 [71].

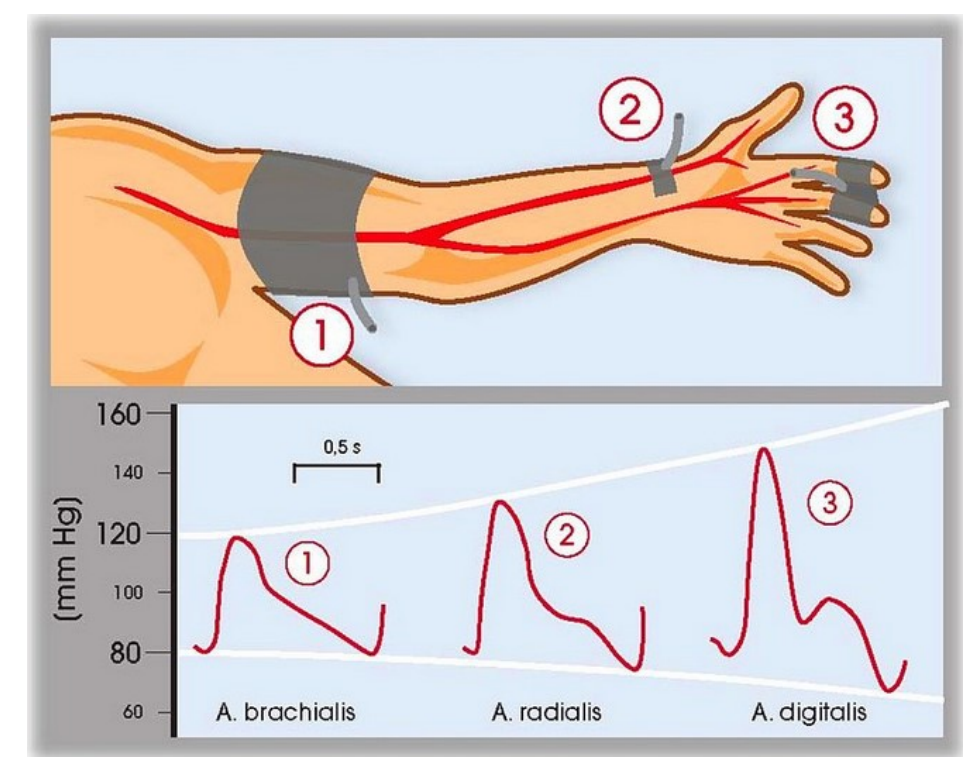

Figure 2.8: Different shapes of blood pressure waveforms [71] 
The ideal method to obtain PWV is invasive. But obtaining PWV invasively is not suitable in clinical examinations or home monitoring. Noninvasive techniques are being developed to measure PWV. Generally, the calculation of PWV for an arterial segment with length $\mathrm{D}$ can be described as:

$$
\mathrm{PWV}=\mathrm{D} / \mathrm{PTT}
$$

where PTT is the pulse transit time, which is the required time for a pressure pulse to travel through the designated arterial segment. So we can also say that PTT is the time when the pressure pulse arrives at the proximal extremity of the arterial segment, subtracting the time when the pressure pulse arrives at the distal extremity of the arterial segment [72], as shown in Figure 2.9.
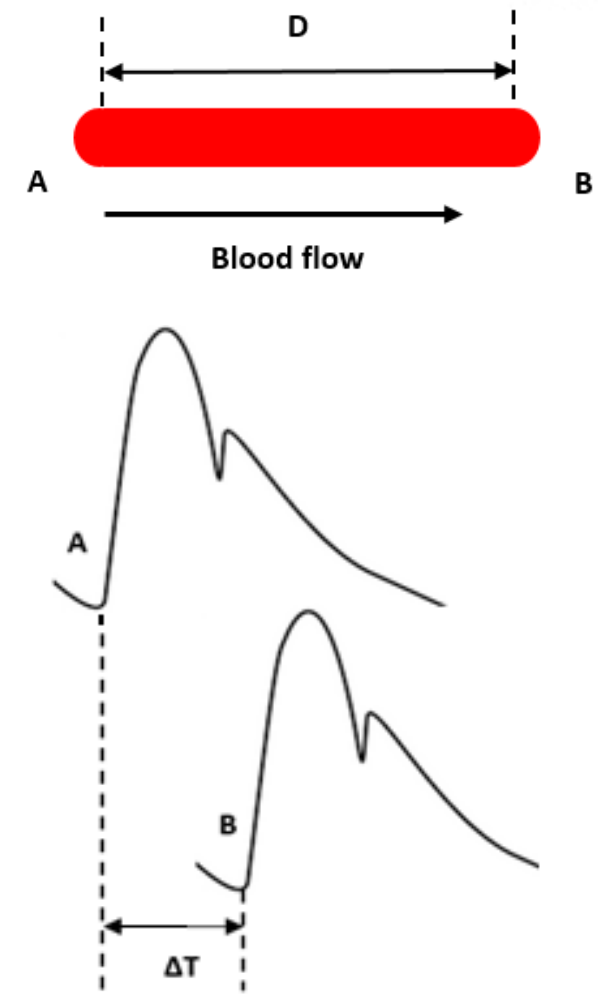

Figure 2.9: Measurement of PTT and PWV 
In Figure 2.9, $\mathrm{A}$ is the proximal extremity of the arterial segment, $\mathrm{B}$ is the distal extremity of the arterial segment, $\mathrm{D}$ is distance traveled by the pulse wave, and $\Delta \mathrm{T}$, namely PTT, is time delay between the foot waves. The foot of the waves is defined as the end of the diastole. In the section that follows, PTT techniques are elaborated on in detail.

\subsection{Pulse Transit Time Technique}

As mentioned in the preceding section, exiting arterial blood pressure measurement techniques based on PWV rely on the measurement of PTT, which is the time required for the blood pulse to travel from one location to another location along the arterial tree. An acute rise in arterial blood pressure will cause an increase in the vascular tone and allow the arterial wall to become stiffer. Therefore, the PTT will be shortened. Conversely, when the arterial blood pressure falls, the vascular tone will decrease and then the PTT will become larger [73]. PTT is inversely proportional to the arterial blood pressure.

PTT has been proven to be a promising index of arterial stiffness and cardiac output. It has been proposed that it is an efficient way to measure the blood pressure noninvasively and continuously, particularly in estimating the arterial blood pressure variations [74]. Also PTT-based techniques are designed in such a way as to avoid complex circuits and highcost sensors [75].

Since the 1970's, a number of different scenarios have unfolded where PTT has been applied as a noninvasive surrogate method to estimate changes in the arterial blood pressure [75]. In 1981, Geddes et al. proved that PTT was closely related to diastolic time when it was detected from different sites along the vessel [76]. PTT was demonstrated to be highly correlated to the arterial blood pressure during dynamic and static exercises by Marie et al. 
[77]. The kinetic energy of the pulse wave and the gravitational potential energy were employed by studying the relationship between the PTT and the arterial blood pressure by Fung et al. in 2004 [78]. Zhang collected continuous blood pressure, ECG signal and PPG signal data from healthy subjects and indicated that PTT could be used to estimate the blood pressure for healthy static body states. The PTT-based technique to wearable devices were also applied [79][80]. In 2006 the Wearable Intelligent Sensor and System for eHealth was proposed [81]. The PTT-based technique was applied to estimate the blood pressure in a wearable multi-parameter remote physiological monitoring system in [82]. Recently, more and more wearable devices have been designed to measure blood pressure variations continuously and conveniently by using PTT [83].

The true value of PTT can be measured by recording the time intervals between two different sites along the passage of the arterial pulse wave. The first group of aortic PTT measurement methods was designed by detecting the arrival of the arterial pulse at two substitute arterial sites that were close to the aorta [84]. It was assumed that the carotid-tofemoral transit time was the best surrogate of the aortic PTT. In this type of measurement an experienced operator was required to manually localize the carotid and femoral arteries and, in addition, the pressure sensors needed to be held during the examination [85].

The second group of aortic PTT measurement methods was based on wave reflection theory. When the arterial pulse travels through the high-resistance arterioles and major arterial branching points from the heart to the periphery, a reflected wave will be created, traveling back from the periphery to the heart. Reference [86] showed that one of the main sources of pulse reflection was the iliac bifurcation at the distal extremity of the descending 
aorta. However, the choice of the reflection point in the arterial tree in this method group has not been identified [87].

To simplify the measurement of PTT, a third group of aortic PTT measurement methods using the R or Q wave of the ECG signal as the starting point and the arrival of the arterial wave detected by photoplethysmograph at a peripheral site as the ending points was developed. It often used at peripheral sites, such as finger tips, ear lobe, and toes. Among these sites, the finger was the most convenient site and was used the most. Usually the ending point was chosen at either $25 \%, 50 \%$, or $100 \%$ of the height of the maximum value of the PPG pulse wave, depending on the equipment used [88]. The PTT measurement using ECG and finger PPG is non-obtrusive, relatively cheap, and portable.

This thesis proposes two strategies to calculate PTT. One is through the time delay between the R-peak of the ECG signal and the following peak of the PPG signal during one pulse cycle. The other one is through the time difference between two areas of interest on the human skin by applying the Eulerian video magnification (EVM) technique. The theoretical backgrounds of the technologies used to calculate the PTT will be provided in the following sections.

The major problem of PTT measurement is that of artifact. The quality of the ECG signal and the finger PPG signal influences PTT in estimating the arterial blood pressure. Electrical noise and movement artifacts could lead to missing or false detection of the Rpeak in the ECG signal. Interference in the finger PPG signal could cause a shift in the PPG primary peak. To overcome this problem, EMD-based algorithms were proposed in this thesis to de-noise the ECG signal and curve fitting algorithms were proposed to detect the primary peak of the PPG signal. In the meantime, we also explored the possibility of using 
EVM, which was recently developed to reveal subtle color changes in human skin, to measure the PTT. The EVM method is video-based and contact-free. No probes or sensors are needed to measure the biomedical signals. The computational complexity of the second strategy, which applied the EVM method, is less than the computational complexity of the algorithms used in the first strategy.

\subsection{Noninvasive Measurement Technologies}

The technologies concerned with signal enhancement in this thesis are ECG, PPG, and Eulerian video magnification. The theoretical background and the previous applications of these technologies relating to noninvasively measure the PTT is elaborated in this section.

\subsubsection{Electrocardiography}

ECG is a diagnostic tool widely used throughout the world to analyze heart conditions. It is a bioelectric signal measured by placing a set of electrodes directly on the skin surface

to observe the electrical activity of the heart. Changes on the skin surface are known to be related to the activity of the heart muscle underneath and the proximity to the heart muscle [89]. The goal of ECG measurement is to detect the ionic current from the skin surface and noninvasively acquire the information on the electrical behavior of the heart.

\subsubsection{ECG Morphology}

A typical ECG signal for one cardiac cycle is shown in Figure 2.10 [90]. A normal ECG signal mainly contains $\mathrm{P}$ wave, QRS complex, and $\mathrm{T}$ wave. The genesis of the ECG waveform through the depolarization and repolarization of the heart is illustrated in Figure $2.11[91]$. 


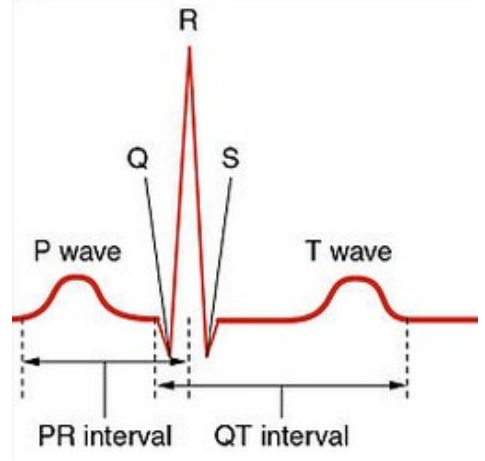

Figure 2.10: A typical ECG signal [90]

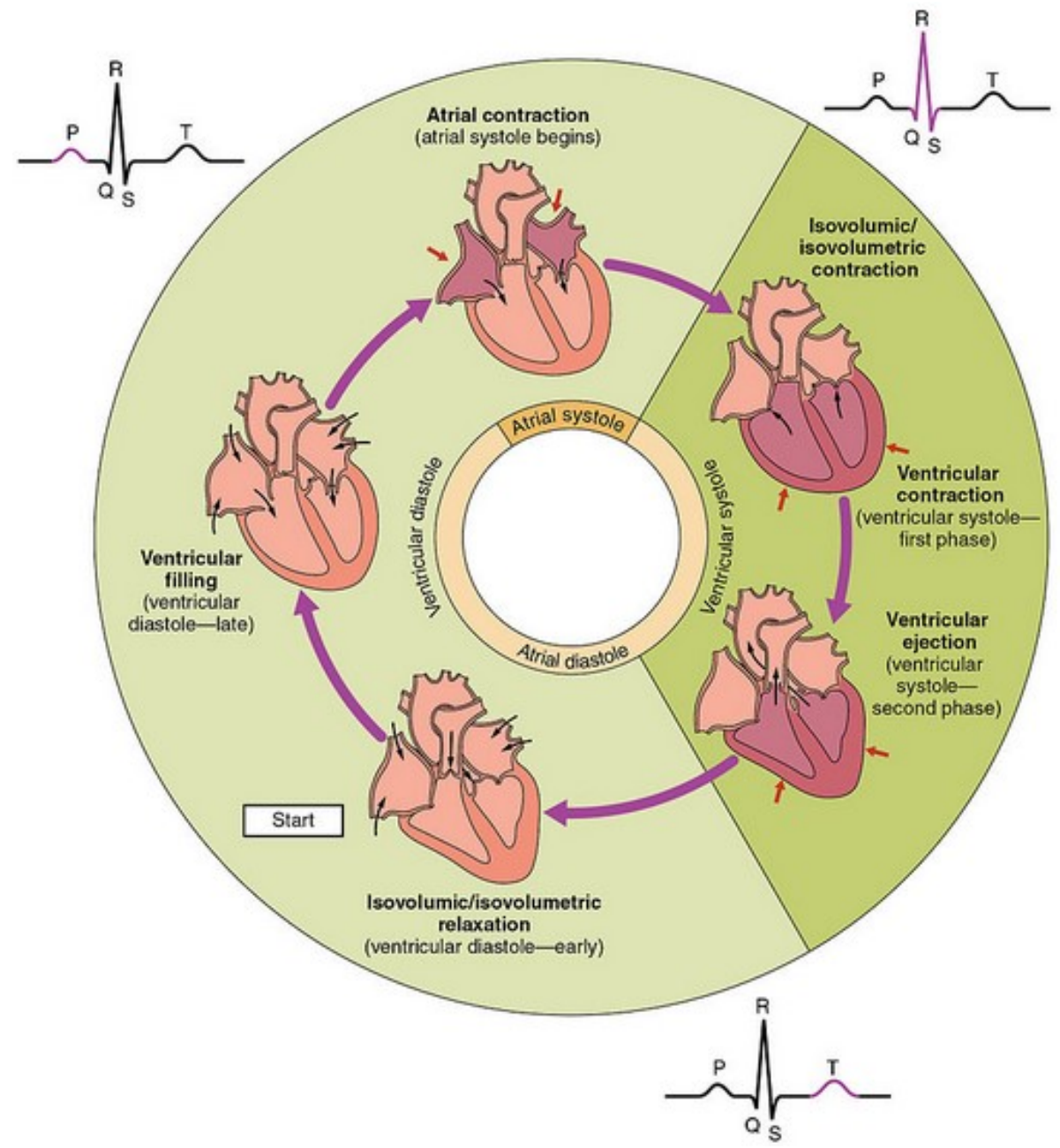

Figure 2.11: Waveform of ECG signal related to the depolarization and repolarization of the heart [91] 


\subsubsection{ECG Measurement}

The electrical current generated through the depolarization and the repolarization of the heart can be measured by placing a set of electrodes on the human body. In clinical practice, a commonly used electrode system is called the 12-lead system. It can differentiate between different cardiac events and consists of a combination of electrodes measuring the activity of the heart muscle from different regions, such as a limb, a precordia, and the chest [92]. Each lead corresponds to the nature of activity on a specific part of the heart muscle [93].

Figure 2.12 illustrates the placement of the ten electrodes and the 12 leads for ECG signals [94][95]. The leads on the limbs are designated as leads I, II, and III and the augmented limb leads are designated as leads aVR, aVL, and aVF. The latter three leads are calculated by combining the signals from the limb leads. Precordial and chest leads are designated as leads V1, V2, V3, V4, V5, and V6.

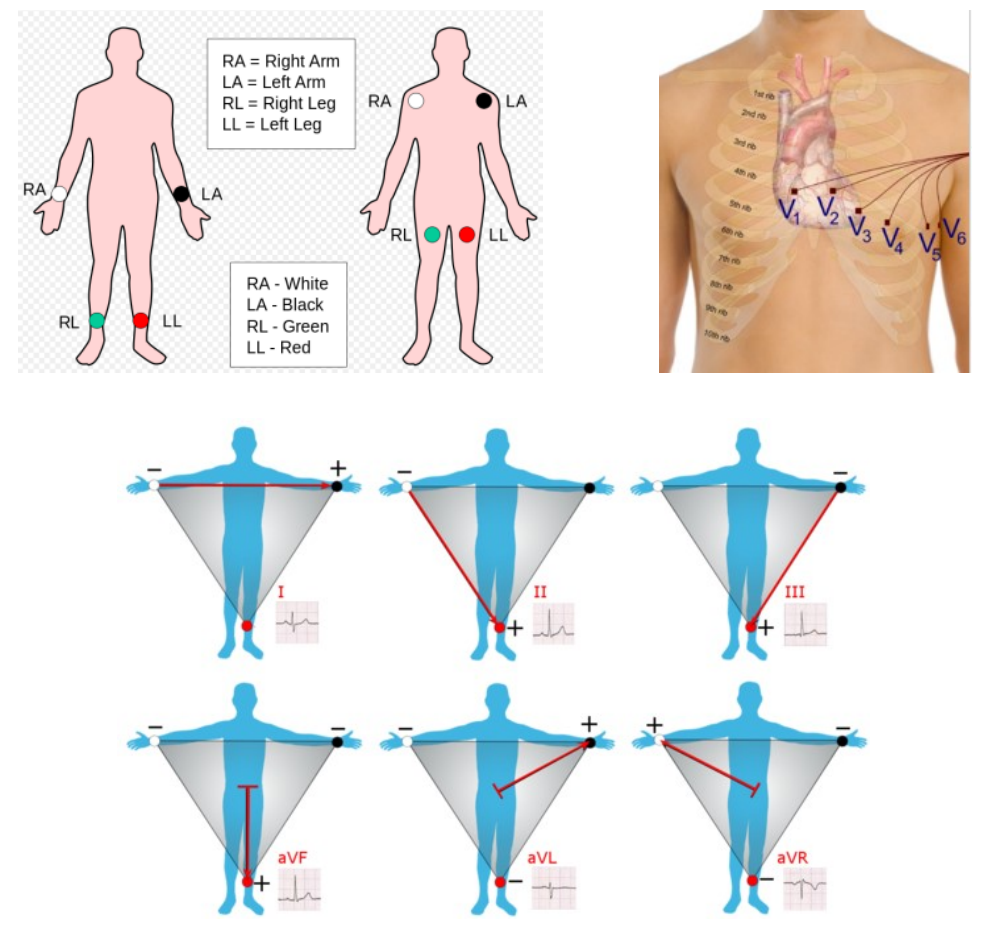

Figure 2.12: Electrodes placement for ECG measurement and the Einthoven's triangle [94][95] 
Each lead represents the electrical potential difference (the voltage) between two points. Leads I, II, and III can be obtained from the following equations:

$$
\begin{gathered}
I=L A-R A \\
I I=L L-R A \\
I I I=L L-L A
\end{gathered}
$$

The augmented limb leads are derived from the same three electrodes that were used for leads I, II, and III. The equations are as follows:

$$
\begin{aligned}
& a V R=R A-1 / 2(L A+L L) \\
& a V L=L A-1 / 2(R A+L L) \\
& a V F=L L-1 / 2(R A+L A)
\end{aligned}
$$

In clinical practice, wet electrodes are used to provide the best possible ECG reading. But when used in continuous self-monitoring, the wet electrodes have some limitations, such as long-term degradation of the electrolyte composition, low regeneration rate, and discomfort caused by the electrode gels. Dry electrodes and textile electrodes have been developed for wearable devices to maintain comfortable self-monitoring [96][97].

\subsubsection{Noises in ECG}

ECG measurements are susceptible to noise because of the small amplitude of the ECG signal on the body surface. For 24-hour continuous monitoring, ECG measurement may be contaminated by different sorts of noise, such as power line interference, baseline wander, electrode contact noise, and muscle artifacts. The noise will corrupt the ECG signal and increase the difficulty in analyzing the ECG signal.

Power line interference occurs for two reasons: capacitive coupling and inductive coupling. Capacitive coupling is defined as the transfer of energy in an electrical network 
by means of the capacitance between circuit nodes [98]. Inductive coupling is generated by mutual inductance between two conductors. Capacitive coupling and inductive coupling are responsible for high frequency noise and low frequency noise, respectively. Depending on the power supply, the value of the power line interference is either $50 \mathrm{~Hz}$ or $60 \mathrm{~Hz}$. To reduce the amount of interference the power line emits, electrodes should be placed properly and all electrical components should be adequately shielded [99].

The variation in the isoelectric line of the ECG signal is called the baseline wander. This is mainly caused by large movement of the limbs and the chest due to respiration or coughing [100]. The range of the baseline wander is between 0 and $0.5 \mathrm{~Hz}$, which is a low frequency signal.

Electrode contact noise is a high amplitude signal compared to the ECG signal. It occurs due to the shifting of the electrodes during exercise. The electrode contact noise usually has a duration of $100-500 \mathrm{~ms}$ and has overlapping frequency contents with the ECG signal [101].

Muscle artifacts occur due to the contraction of other muscles during ECG acquisition. It has been proven that the electromyographic (EMG) signal has a stochastic amplitude and a mean of zero. The frequency of the muscle artifacts is between $100 \mathrm{~Hz}$ and $500 \mathrm{~Hz}$ [101].

\subsubsection{ECG Database}

The ECG signal used in the ECG signal enhancement section was from the MIT-BIH Arrhythmia Database, which was setup by Massachusetts Institute of Technology (MIT) and Beth Israel Hospital (BIH) [102]. The database contains 48 excerpts of two-channel ambulatory ECG recordings (lead II and lead V1). Each recording is digitized at $360 \mathrm{~Hz}$ with 11-bit resolution over $10 \mathrm{mV}$ range and has a duration of 30 minutes [103]. 
Two types of noise were considered for ECG enhancement: Gaussian white noise and contact noise. Gaussian noise was used as a test experiment, and was generated by MATLAB. Gaussian noise was added to the original ECG signal with the signal-to-noise ratio (SNR) of $10 \mathrm{~dB}$. The other type of noise was actual noise from the MIT-BIH Noise Stress Test Database [104]. This database contains three types of noise that usually appear in ECG signals, like baseline wander, muscle artifacts, and electrode contact noise. All the recordings were taken from standard recorders and healthy subjects. Two noise recordings were employed, the muscle artifact, which was noted as "ma", and the electrode contact noise, which was noted as "em". In order to compare the de-noising performance to the previous research, we combined the muscle artifacts and the electrodes contact noise together with same contribution to contaminate the original ECG signal. Therefore, the noise applied could be described as [105]:

$$
n(t)=k_{1} n_{m a}(t)+k_{2} n_{e m}(t)
$$

\subsubsection{ECG De-noise Algorithms}

Efficient algorithms and techniques are needed to remove the aforementioned interference existing in the ECG signal, especially to recover the original ECG signal corrupted by the motion and activities of the patients. There has been some research done working on the enhancement of the ECG signal, such as nonlinear filter banks [106], adaptive filtering [107], the wavelet transform [108], and independent component analysis (ICA) [109]. For instance, sinusoidal noise was used as the reference input for reducing the contact noise in [110]. More complicated noise was considered in [111].

Among the above-mentioned signal enhancement methods, adaptive filtering techniques were mainly used. The basic concepts and applications of the adaptive filtering 
techniques were first described by Widrow [112] and have been used in many ECG signal processing problems [113][114]. One important application was extracting the fetal ECG from the maternal ECG. The input obtained from the chest leads was regarded as the reference input containing the maternal ECG and the power-line interference. Different adaptive filtering structures were proposed for noise cancellation and arrhythmia detection according to diverse types of noise in [107]. Besides adjusting the filter coefficients with fixed filter order, J. Lee and G. Lee developed an adaptive filter with a dynamic structure to make the filter order adaptable [115]. In [116], a light-emitting diode and an optical sensor incorporated into an ECG electrode were used to obtain the reference signal of the adaptive filter. The wearable system and method had certain capability to reduce muscle artifacts and electrode contact noise for ambulatory ECG monitoring, but the accuracy of the muscle strain measurement could not be guaranteed.

Though there have been many advanced signal processing methods applied to denoise the ECG signal, the research on ECG enhancement is still largely open due to its unique difficulty. Traditional signal enhancement and noise reduction algorithms are almost all based on standard filter processing. Due to the de-noising process, some useful components of the ECG signal itself, which may contain very important information about the health status of the patients, may be removed or reduced as well.

The filter-bank based de-noising technique smooths the amplitude of the P-wave and the QRS-complex of the ECG signal and it is more sensitive to different levels of noise [117]. The limitation of the adaptive filtering based ECG de-noising lies in the fact that a reference signal has to be additionally recorded together with the ECG [107]. In wavelet transform, the ECG signal is analyzed and expressed as a linear combination of the sum of 
the product of the wavelet coefficients and the mother wavelet [118]. The mother wavelet and the cut-off frequencies of a series of low-pass and high-pass filters needs to be determined ahead of time. A statistical model of the ECG signal and noise was first extracted in ICA or neural networks (NN), and then the in-band noise was removed by discarding the dimensions corresponding to the noise in [119]. The derived statistical model is not only fairly arbitrary, but also extremely sensitive to small changes in either the signal or the noise. To conquer this problem, the basic functions have to be trained on a global set of ECG beat types. One of the difficulties with the application of ICA is the determination of the order of the independent components [119].

A recent algorithm called Empirical Mode Decomposition (EMD) has been developed as a technique for processing nonlinear and non-stationary signals [120]. This method was originally used for the study of blood pressure in [121]. In [122], EMD was used to investigate the chaotic nature of ECG signals. Blanco-Velasco developed an EMDbased method to remove the baseline wandering and high-frequency noise of ECG signals in [105]. Compared to other de-noising methods, such as filter banks, ICA, NN, adaptive filtering, and wavelet de-noising algorithm, EMD was found to be more effective in reducing noise from the ECG signal [123].

The advantage of EMD is that it is intuitive and adaptive, with basic functions derived fully from the data. The computation of EMD does not require any previously known value of the signal. The key task is to empirically identify the intrinsic oscillatory modes by their characteristic time scales in the signal, and accordingly, decompose the signal into intrinsic mode functions (IMFs) [120]. Therefore, EMD is especially applicable for nonlinear and non-stationary signals, like ECG. 
Ensemble EMD (EEMD) is an improved EMD algorithm, and was originally developed to reduce the mode-mixing effect between the next two IMF scales [124]. When the high frequency components of the signal have intermittence or the frequencies of the next two IMFs lie close to each other, the mode-mixing problem will arise. Chang compared the de-noising results between EMD and EEMD and used EEMD to enhance the arrhythmia ECG signals [125][126]. The method in [125] and [126] used the partial reconstruction method, which meant that several IMFs were removed directly. Z. Zhao proposed a method which combined EMD and adaptive filtering to remove the power-line frequency and baseline wander in ECG signals [127][128]. Results demonstrated that the proposed method was very useful, and both power-line interference and baseline wander could be reduced efficiently, but contact noise was not taken into consideration. In [60], we designed an improved EEMD-based method to enhance ECG signals, and the results demonstrated that the contact noise could be efficiently reduced.

Details of the EMD-based algorithms and the methods proposed in this thesis will be discussed in Chapter 3.

\subsubsection{Photoplethysmography}

PPG is an optical technology which noninvasively assesses the information on the subcutaneous blood circulation. PPG has been widely used in a clinical setting in monitoring heart rate, respiration, depth of anesthesia, and hypo- and hypervolemia. The principle of PPG technology involves illuminating the tissue with an infra-red light source and analyzing the changes in absorption caused by the arterial pulse. The arrival of the arterial pulse can be detected through the temporal series of the light absorption [129]. In 
the current thesis, PPG technique is used to assess the arrival time of the arterial pressure pulse. Then PTT can be measured from the enhanced ECG signal and PPG signal.

\subsubsection{Principle of PPG}

When skin is exposed to light, the light will be reflected, absorbed, or transmitted. The human tissue bed consists of different components, such as blood, bone, blood vessels, tissue, fluid, and skin. Different components have unique optical properties and different absorption rates. Generally, PPG relies on three aspects [130]. One aspect is illuminating the tissue at the area of interest with a light beam. Another is capturing a portion of the light which has propagated through the tissue. The last is analyzing the captured light and illustrating the structural information on the tissue.

The Beer-Lambert law was adopted to establish a model for understanding the principle of PPG [131]. The equation used to depict the Beer-Lambert law is as follow:

$$
I=I_{b} e^{-\sum_{N} \omega_{n} l_{n}}
$$

where $I$ is the intensity of the transmitted light through the tissue. $I_{b}$ is the intensity of an injected light beam. $N$ is the number of absorbing optical segments that the tissue at the area of interest has. $l_{n}$ is the optical path length and $\omega_{n}$ is the light absorption coefficient for each optical segment.

In one heart cycle during systole, the blood is pumped from the heart to the body. The increase of blood results in more light absorption. During diastole, the blood fills the ventricles. The decrease of the blood in the arteries results in less light absorption. Therefore, the PPG waveform primarily contains two components: a DC component and an $\mathrm{AC}$ component. The $\mathrm{DC}$ component is a slowly varying baseline and depends on the amount of light that is absorbed from different types of tissue. The DC component changes 
with respiration, while the $\mathrm{AC}$ component is varying, depending on the volume of blood flow through the arterial blood vessel [132][133]. The fundamental frequency of the AC component is the same as the heart rate.

Usually there are two peaks in one cycle of PPG signal. The primary peak happens when the artery distends due to blood flow. The secondary peak (smaller peak) occurs during the time when the blood flows back to the heart through the veins.

\subsubsection{PPG Measurement}

The PPG signal is measured using a pulse oximeter which illuminates the skin and detects the changes in light absorption. Common light sources used in PPG measurement are Light Emitting Diodes (LED) or Laser [130]. The chosen light wavelength is between 600 and 1300nm [134]. Basically, based on the positions of the light source and the photodiode, there are two types of modes of PPG measurement, namely the reflectance mode and the transmission mode. Figure 2.13 is a simple illustration of the reflectance mode and the transmission mode PPG.

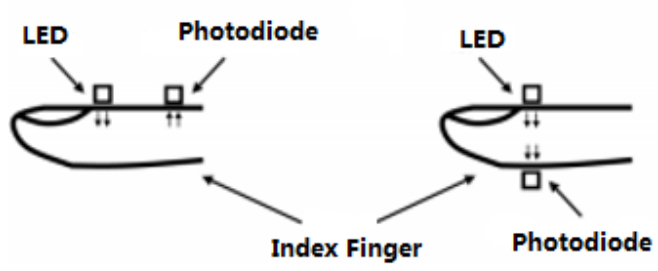

Figure 2.13: Illustration of the reflectance mode and the transmission mode PPG

In reflectance mode, the LED and the photodiode are placed adjacent to each other on the skin surface. The photodiode detects the light back-scattered or reflected from the skin. The intensity of the back-scattered or reflected light displays the information on the changes of the blood volume. The reflectance mode PPG measurement is often used on a 
flat skin surface, such as chest, forehead, or cheek, places where the transmission mode is not suitable. To measure the PPG signal accurately, the sensors should be attached firmly to the skin surface and an opaque shield is needed between the LED and the photodiode.

In transmission mode, the LED is place on one side of the tissue and the photodiode is placed on the opposite side to gather the transmitted light through the tissue. This mode is usually used on the fingertip, earlobe, or toe, where the transmitted light can be detected. The photodiode needs to be placed as close as possible to the skin surface to avoid any exerting force.

\subsubsection{Peak Shifting Phenomena}

The PPG signal is very sensitive to movement and susceptible to erroneous recordings, especially when the perfusion is low. Generally there are two peaks in one PPG cycle. The peak with the greater amplitude is defined as the primary peak and the peak with the smaller amplitude is the secondary peak. The secondary peak will become invisible due to movement [135]. When the patients are older adults or have cardiovascular disease, the secondary peak will easily be embedded in the whole PPG signal. In order to demonstrate the influence of the secondary peak in measuring PTT, the peak shifting phenomenon is briefly depicted in this section.

Taking Gaussian curves as an example, Figure 2.14 illustrates two of the many scenarios in which the primary peak shifts and the secondary peak becomes invisible after combining the two curves together. More scenarios of peak shifting problems will be illustrated in Chapter 4.

The scenario in Figure 2.14 (a) is that the two curves too close to each other. Figure 2.14 (b) shows the scenario in which the difference of the amplitude of the two Gaussian 
curves is too large. It is obvious that the position of the primary peak has been shifted to the right and the secondary peak disappears under both conditions. Since we measure the PTT as the delay between the R-peak of the ECG signal and the following primary peak of the PPG signal, the shifting of the primary peak will influence the accuracy of PTT measurement.

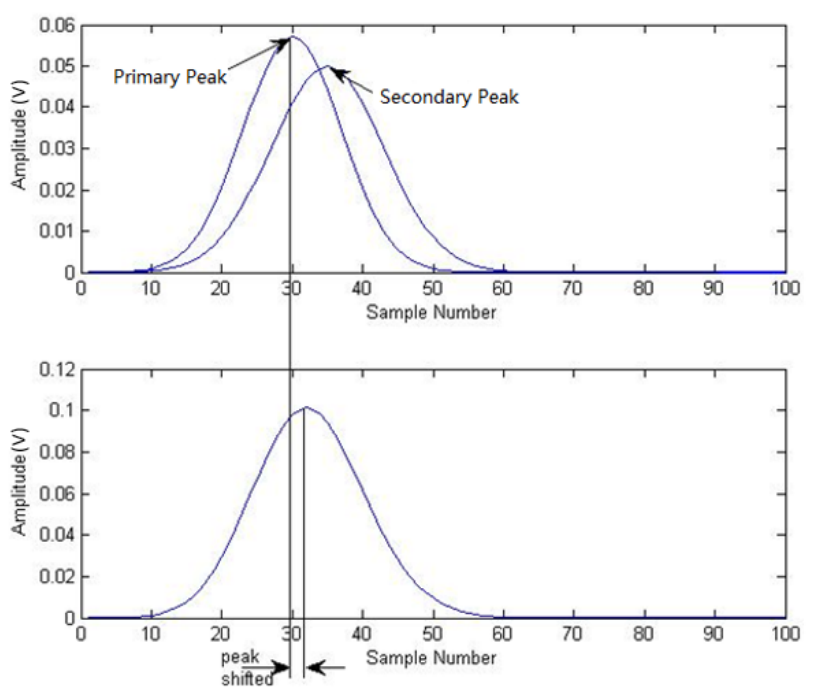

(a)

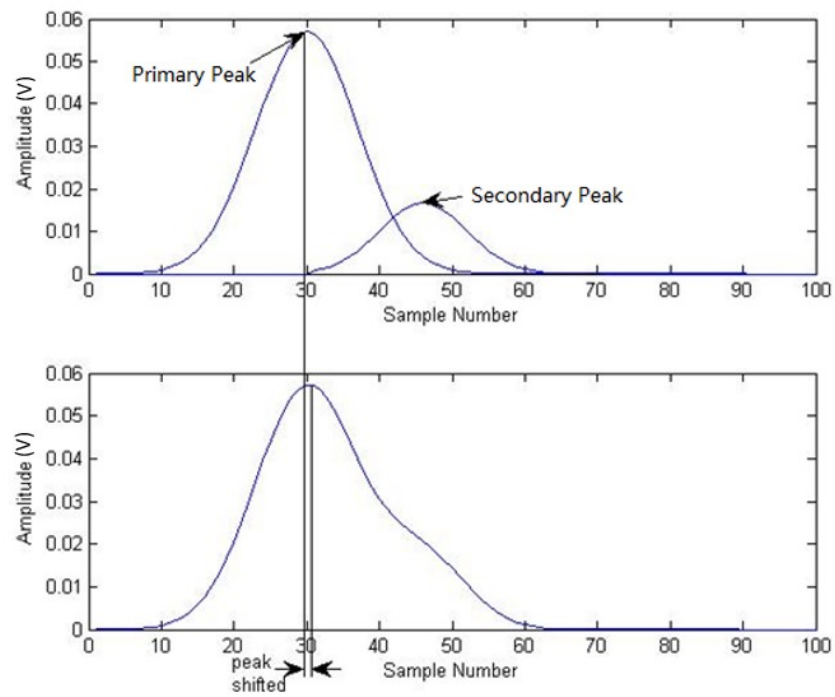

(b)

Figure 2.14: Peak shifting phenomenon illustrated by the combination of two Gaussian curves 


\subsubsection{PPG Database}

The PPG signal used in this thesis is from the MIMIC II database. The MIMIC II database contains time series of clinical signals and vital signs obtained from hospital medical information systems. The signals in the MIMIC II database were multi-parameter recordings, which were obtained from both a bedside monitor and the medical records of the patient [136]. Clinical data was obtained from the CareVue Clinical Information System (models M2331A and M1215A; Philips Health-care, Andover, and MA), as well as from hospital electronic archives [137]. So the measurement instruments used to acquire the biomedical signals in this part is real clinical devices. The algorithm we proposed does not only work on some specified cases, but also for most of the clinical cases.

The signals used for the simulations are selected from the clean portions of the patient's record. So the signals are clear enough for to detect the feature points. The tool used to extract the signals from the database is called PhysioBank ATM, which has several toolboxes, including plotting the waveforms of one specific signal, showing samples as text, and exporting signals as .mat [138].

\subsubsection{PPG Peak Detection Algorithms}

Due to respiration, contact noise, motion artifacts, and the physiological variability of the PPG signal, the detection of the PPG peaks is difficult in practice [130]. Recently, some PPG peak detection algorithms were developed. An EMD-based signal processing method on correctly extracting the heartbeat was proposed in [139]. It was mentioned that the heartbeat information embedded in the PPG signal was one of the most important physiological observations in clinical and mobile health monitoring. The accuracy of the heartbeat detection was improved and the detection rate was $84.68 \%$. Wavelet and 
correlation methods were demonstrated to have the ability to determine the heart rate. The fuzzy logic discriminator was used to extract pulse peaks in the slope of the PPG signal [140].

An automatic peak detection algorithm was designed in [141]. The first peak following each heartbeat was detected for pressure signals. The algorithm consisted of a filter bank, nonlinear filters, heart rate spectral estimation, and the decision logic. The importance of PPG detection was emphasized in [142]. The paper showed the diagnosis of twenty physiological and cardiovascular diseases based on PPG temporal analyses, which mainly relied on the PPG peak points.

There is also some research that looked into the dictoric notch of the PPG signal. Dicrotic notch is a small downward deflection that separates the systolic and diastolic phase. The location of the dicrotic notch is used to calculate systolic time intervals and monitor cardiac function [143]. The dicrotic notch plays an important role in evaluating various diseases in the early stage.

A parameter from PPG dicrotic notch, called relative amplitude of secondary peak (RAS), was used for the estimation of systolic blood pressure in [144]. The result showed that the bias underestimated could be largely reduced by introducing RAS. Hyun and his colleges proposed a method for blood pressure estimation using multiple-regression with pulse arrival time (PAT) and two other confounding factors: heart rate and the duration of the maximum derivation point to the maximum of the dicrotic notch in the PPG signal [145].

In this thesis, three secondary peak detection methods are proposed to accurately detect the peak locations of the PPG signal. 


\subsubsection{Eulerian Video Magnification}

Nowadays, both the speedy development of cell phone technology and the markets have driven the cost of cameras down. This essentially means that cameras could be used as a very inexpensive bio-sensor under well-designed algorithms. A novel method, called Eulerian video magnification (EVM), was proposed recently by Rubinstein and his colleagues [146]. The principle of EVM is to reveal the subtle changes, such as color or motion which are impossible to detect with the human eye, through the usage of cameras.

\subsubsection{Principle of EVM}

The Eulerian framework is designed for fluid flow analysis. The Eulerian video magnification method is similar to the Eulerian framework. It was developed to observe and manipulate temporal variations at specific spatial locations. Compared to Lagrangian approaches, the Eulerian approaches do not explicitly estimate motions. They calculate non-motion compensated frame differences to estimate subtle variations [147]. So EVM is able to not only magnify motion changes, but also to magnify color changes.

The principle of the EVM is to visualize temporal variations that are impossible to see with the naked eye and re-render a video to display these variations. The input of the EVM is a standard video sequence. After applying spatial decomposition and temporal filtering to the frames, the filtered signal is then amplified to reveal the subtle changes, like the color changes on different locations caused by the pulse. With this method, not only can the heart rate be easily obtained, so can the moment when the flow of blood goes through certain spots of interest can be easily obtained.

Figure 2.15 shows the framework of the EVM. The input video is first decomposed into different spatial frequency bands. These frequency bands may be differently magnified 
according to their different signal-to-ratio values. Temporal processing is then applied on each frequency band. The frequency bands of interest are extracted by a band-pass filter. A factor $\alpha$ is chosen to magnify the extracted frequency bands. The selection of $\alpha$ is determined by specific application. The magnified signal is eventually added to the original video to rerender the output.

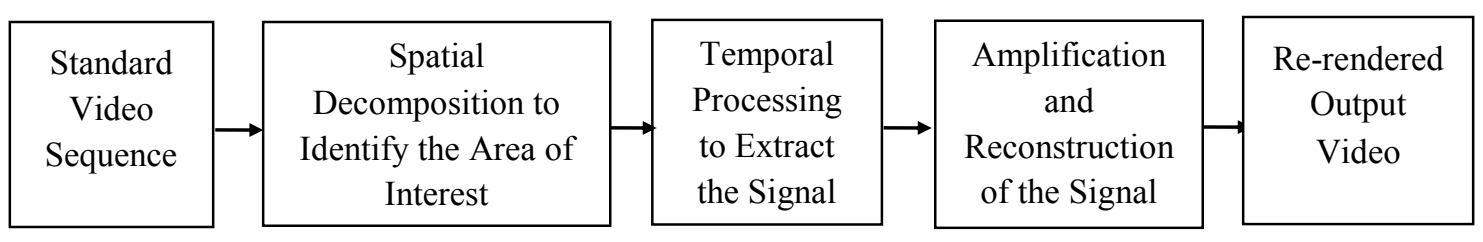

Figure 2.15: Framework of the Eulerian video magnification method

\subsubsection{Spatial Processing}

In the spatial processing part, the frames of the input video are decomposed to obtain a spatial pyramid. Spatial filtering can enhance the subtle changes in the signals if the used spatial filter used is large enough to reveal the signal of interest. The size of the spatial filter can be estimated through the spatial characteristics of the signal. The variance of the noise can be reduced by the spatial low-pass filtering according to the area of the low-pass filter. The estimated size of the spatial filter used to visualize the signal under certain noise can be described as [146]:

$$
S(r)=\sigma^{\prime 2}=k \sigma^{2} / r_{l p}^{2}
$$

where $S(r)$ represents the signal power over spatial frequencies. $\sigma^{\prime 2}$ is the filtered noise power level. $k$ is a constant depending on the shape of the low-pass filter. $\sigma^{2}$ is the noise power estimated in a stable region and $r_{l p}$ is the radius of the spatial low-pass filter. From 
equation (2.8), it can be seen that the filtered noise power level is inversely proportional to the radius $r_{l p}$, within which the signal power is greater than the noise.

\subsubsection{Temporal Processing}

After the pre-processing of the spatial filtering, the temporal processing is performed on each spatial band. Temporal filtering is used to extract the subtle changes to be amplified. The choice of the filter is determined case by case. A broader band-pass filter is more suitable for motion magnification while a narrow band-pass filter is mainly employed for color magnification [147]. If it is for heart rate detection, the selected frequencies of the band-pass filter could be within $0.8 \mathrm{~Hz}$ and $3 \mathrm{~Hz}$. The temporal processing is uniform for all pyramid levels and for each pixel in each level.

\subsubsection{EVM Applications}

Although EVM is a new method and was first proposed in 2012, there have been some applications with EVM. The second EVM technique based on the observation of the phase variations corresponding to motion was developed in 2013 [148]. The phase-based technique has been demonstrated to have better de-noising performance than the linear technique. In [149] EVM was used for processing video sequences obtained from a standard video camera for pulse rate assessment. The results showed the requirement of a high quality camera for the usage of EVM. An enhanced EVM was proposed in [150]. A post-processing technique was introduced to EVM. The proposed processing was shown to be able to support larger amplification and was more independent of the frame noise. EVM was used to extract micro-expression on the face to detect the emotions of the subjects in [151]. EVM was applied to Histogram of Oriented Gradients (HOG) features and Back-propagation Neural Network. 
In the current thesis, EVM is applied so that the periodic color changes caused by the blood pulse at different areas on the human skin become visible to the human eye. Due to the blood flow, human skin color changes slightly. The changes of the skin color provides useful information about human cardiovascular system [152]. But this color variation is below the detection capacity of the human visual system, which means the variation is invisible to the naked eye. By detecting the pulse arrival time at two different locations, PTT can be obtained. EVM is considered as an invasive and contact-free method to continuously measure the PTT, which triggers the interest in the development and commercialization of medical devices implementing the EVM method.

\subsection{Summary}

This chapter described the background of the arterial blood pressure measurement and the PTT technique. Two strategies were introduced for the PTT measurement. By defining the PTT as the time difference between the peak of the R-wave of the ECG signal and the primary peak of the following PPG signal in one pulse cycle, one strategy was to improve the accuracy of the PTT measurement through enhancing the ECG signal and the PPG signal. The other strategy was applying a video processing framework, namely Eulerian video magnification, to measure the PTT directly. Details on the two strategies will be explained in the following chapters, respectively. 


\section{Chapter 3}

\section{ECG Enhancement using EMD-based Algorithm}

\subsection{Introduction}

The morphologic analysis of electrocardiogram (ECG) signals is an important standard for the diagnosis of heart diseases and other pathological phenomena. The ECG signal, however, is always contaminated with different types of noise, especially when the sensor is worn by patients during their normal activities, where the muscle and motion artifact is the dominant noise.

This chapter proposes a new ECG enhancement method, which is based on Ensemble Empirical Mode Decomposition (EEMD) and the adaptive filtering techniques, to eliminate the contact noise contained in ECG signals. The reference signal of the adaptive filter is produced by the selective reconstruction of the decomposition results of the EEMD method. The performance of the proposed method is validated by using real data from the MIT-BIH database. Simulation results show that ECG signals from wearable monitoring sensors can be significantly enhanced by filtering out the contact noise while all the ECG features are kept. The EEMD-Adaptive (EEMDA) method exhibits obvious advantages over other similar ones in terms of de-noising. 


\subsection{Overview for EMD-based Algorithm and Adaptive Technique}

EMD is a technique to separate an arbitrarily given signal into a sum of intrinsic mode functions (IMFs). The IMFs act as a naturally derived set of elemental functions for the signal, so EMD could be regarded as an exploratory data analysis technique [153]. Actually, EMD and the Hilbert-Huang transform are the basic components of the "Hilbert Spectral Analysis" [117].

\subsubsection{Instantaneous Frequency and IMFs}

Given an arbitrary time series $X(t)$, its Hilbert transform $Y(t)$ is as:

$$
Y(t)=\frac{1}{\pi} P \int_{-\infty}^{\infty} \frac{X\left(t^{\prime}\right)}{t-t^{\prime}} d t^{\prime}
$$

where $P$ stands for the Cauchy principal value.

$X(t)$ and $Y(t)$ form a complex conjugate pair, so we can have an analytic signal $Z(t)$ as:

$$
Z(t)=X(t)+i Y(t)=a(t) e^{i \theta(t)}
$$

where $a(t)=\left[X^{2}(t)+Y^{2}(t)\right]^{1 / 2}$ and $\theta(t)=\arctan \left(\frac{Y(t)}{X(t)}\right)$. The instantaneous frequency is defined as:

$$
\omega=d \theta(t) / d t
$$

Equation (3.3) shows that the instantaneous frequency is a single value function of time, so there is only one frequency value corresponding to each time point. The necessary conditions for us to define a meaningful instantaneous frequency are, that the functions are symmetric with respect to the local zero mean, and have the same numbers of zero 
crossings and extrema [117]. So Huang proposes a class of functions designated as intrinsic mode functions.

An IMF is defined as a function with an equal number of zero crossings and extrema, or differs at most by one; and the mean value of the envelope defined by all the local maxima and the local minima is zero. Each IMF represents an oscillatory mode of the signal. IMF distribution is very similar to a filter bank, but it is not band restricted, which is not same as the traditional filter bank. Comparing to the wavelet transform algorithm, the main feature of EMD-based method is that the decomposition is adaptively and is totally based on the signal pattern complexity.

\subsubsection{EMD Algorithm}

The decomposition process used in EMD is called the sifting algorithm. The steps of the process are as follows:

Step 1) Find all the local maxima and minima of the signal $x(t)$;

Step 2) Connect all the local maxima by a cubic spline curve as the upper envelope $e_{\text {upper }}(t)$, and connect all the local minima by a cubic spline curve as the lower envelope $e_{\text {lower }}(t)$;

Step 3) Subtract the mean of the two envelopes $m_{1}(t)=\left[e_{\text {upper }}(t)+e_{\text {lower }}(t)\right] / 2$ from the signal and get the first proto-IMF $h_{1}(t)=x(t)-m_{1}(t)$;

Step 4) Repeat the sifting process (1) to (3) on $h_{1}(t)$ until the resulting proto-IMF, which is named as IMF1 $c_{1}(t)$, satisfies the IMF conditions. Some stopping criteria are used such as:

$$
\sum_{t=0}^{T} \frac{\left|h_{k-1}(t)-h_{k}(t)\right|^{2}}{h_{k-1}^{2}(t)} \leq S D
$$


The value of SD is usually set to $0.2-0.3$.

Step 5) Subtract the IMF1 from the original signal. The residual is $r_{1}(t)=x(t)-c_{1}(t)$;

Step 6) Repeat step (1) to (5) to obtain other IMFs until the residual of step (5) is a constant, a monotonic slope, or a function with only one extremum. The last residual is the trend.

The EMD of the original signal can be represented as:

$$
x(t)=\sum_{n=1}^{N} c_{n}(t)+r_{N}(t)
$$

\subsubsection{EEMD Algorithm}

The principle of EEMD is to add white noise into the original signal with several trials. The added noise is different in each trial, and can be canceled out on an average, under the condition that the trial number is high enough. Then the ensemble result is the original signal as more and more trials are added [119]. Although the basic decomposition process of EEMD is the same with EMD, the improved ensemble process can reduce the modemixing problem that exists in the EMD algorithm. The process steps for the EEMD algorithm are as follows:

Step 1) Add white noise to the original signal to obtain $x_{i}(t)=x(t)+n(t)$;

Step 2) Use the EMD algorithm to decompose $x_{i}(t)$;

Step3) Repeat step (1) and (2) $m$ times, until $m$ reaches the given trial number. Add different white noise of the same power in each loop. The IMFs $c_{i j}(t)$ is the new IMFs, where $i$ presents the iteration number and $j$ is the IMF scale;

Step 4) Calculate the mean of the final IMF:

$$
c_{j}=\sum_{i=1}^{m} c_{i j}(t)
$$




\subsubsection{Adaptive Filtering Technique and LMS}

The basic idea of the adaptive filtering technique was first introduced by Widrow. Depending on the resource of the reference input, an adaptive filter normally has two consitions [107]. The first condition is that the reference input is the noise correlated in some extent with the real noise contained in the original input signals. The other condition is that the reference input is a noise-free signal, which can be used to minimize the meansquared error (MSE) between the original signal and the reference input. Under both conditions, the essence of the adaptive filtering is the same: time-varying and self-adjusting.

If the original input signal is $x$ with noise $n$, the reference input noise is $n_{r}$, the output of the adaptive filter is $y$, and the filter error is $e$, then the MSE can be calculated as:

$$
E\left[e^{2}\right]=E\left[(s+n-y)^{2}\right]
$$

As the pure signal and the noise are uncorrelated, the cross term $2 E[s(n-y)]$ is zero. Therefore equation (3.7) can be simplified as:

$$
E\left[e^{2}\right]=E\left[(n-y)^{2}\right]+E\left[s^{2}\right]
$$

To obtain the best estimation of the pure signal is to minimize the MSE of the filter error, which is to minimize the MSE between the noise and the output of the adaptive filter.

The most widely used adaptive algorithm is the least-mean-squares (LMS) algorithm. It is an iterative algorithm to minimize the MSE between the original signal and the reference input by adapting the filter coefficients. The general equation of LMS is as:

$$
\begin{gathered}
\mathbf{W}_{k+1}=\mathbf{W}_{k}+2 \theta e_{k} \mathbf{N}_{r k} \\
y_{k}=\mathbf{W}_{k}^{T} \mathbf{N}_{r k} \\
e_{k}=s_{k}+n_{k}-y_{k}
\end{gathered}
$$


where $\mathbf{W}_{k}$ is the vector of filter coefficients and $\mathbf{N}_{r k}$ is the reference input vector. $\theta$ is the step size which controls the balance between the convergence rate and the system stability.

\subsubsection{EEMD-Adaptive Algorithm}

There are two ways to improve the performance of the adaptive filtering in ECG enhancement. One is to modify the adaptive filtering algorithms or structures, such as the sign LMS algorithm, the normalized LMS algorithm, the error normalized LMS algorithm, and the cascade adaptive filtering structure. The other way is to find an efficient and easy method to obtain more useful and precise reference input for the adaptive filter, as mentioned in references [111], [127], and [128]. As for the advantages of EEMD method in analyzing nonlinear and non-stationary signals and the adaptive filtering technique in dealing with time-varying signals, we combined these two algorithms together to enhance noisy ECG signals without bringing distortion to the original signals. The diagram of the EEMDA method is shown in Figure 3.1.

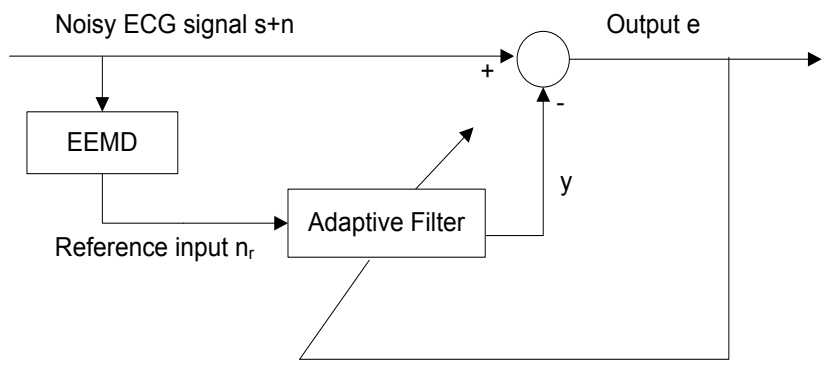

Figure 3.1: The scheme of EEMDA algorithm

The reference input in Figure 3.1 is obtained from the selective reconstruction of the EEMD output. The details of the reconstruction processing are described in the following section. 


\subsection{Signal Enhancement}

\subsubsection{ECG Signal and Noise Signal}

The ECG signal used in this study is from the Arrhythmia ECG Database in the MIT-BIH Database [102]. Each record was digitized at $360 \mathrm{~Hz}$ with 11-bit resolution. Five records are chosen, each record containing 30-minute duration.

Clean ECG signal

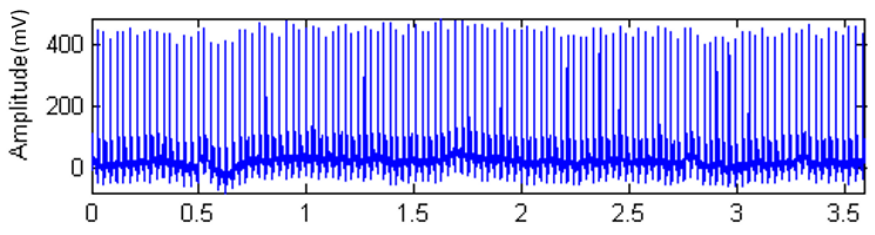

Noisy signal with SNR value of $10 \mathrm{~dB}$

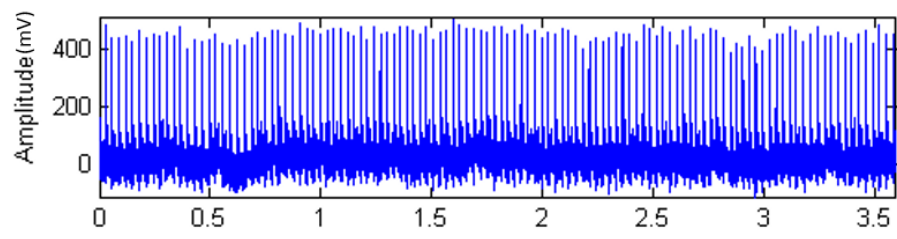

Muscle artifacts noise

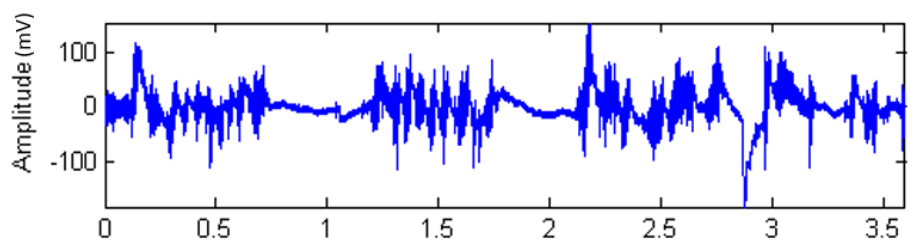

Motion artifacts noise

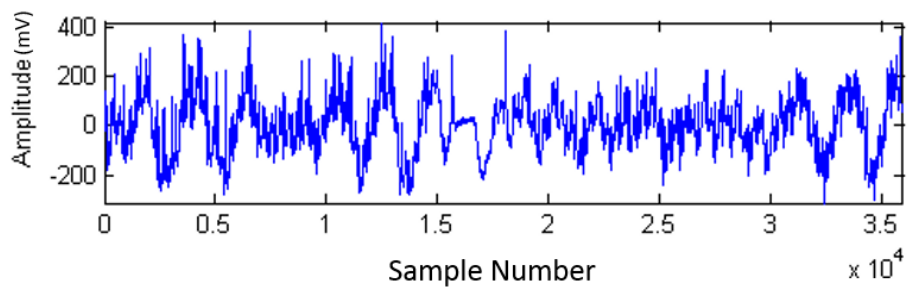

Figure 3.2: The original ECG signal and noise used in this chapter 
ECG signals usually contain two types of noise, Gaussian white noise and contact noise. Gaussian noise is used as a test experiment, and is generated by MATLAB. Gaussian noise is added to the clean ECG signal with the signal-to-noise ratio (SNR) of $10 \mathrm{~dB}$. The other kind of noise is the real noise from the MIT-BIH Noise Stress Test Database. Two noise records are taken, the muscle artifacts, which is recorded as "ma", and the electrode motion artifact, which is recorded as "em". In order to compare with the previous work, we combine the muscle and motion artifacts together with the same contribution to corrupt the clean ECG signal. So the total noise is $n(t)=k_{1} n_{m a}(t)+k_{2} n_{e m}(t)$ [105].

Take the first lead of record 103 as an example, Figure 3.2 showing the segment of the clean ECG signal, the ECG signal contaminated by Gaussian white noise with an SNR value of $10 \mathrm{~dB}$, and the contact noise.

\subsubsection{Decomposition Performance of EMD and EEMD}

One major drawback of the original EMD is the frequency appearance of mode mixing, which is defined as a one IMF consisting of signals of widely disparate scales, or a signal of a similar scale residing in different IMF components. Mode mixing is a consequence of signal intermittency [124]. In order to demonstrate that EEMD has the ability to reduce the mode-mixing impact exists in EMD, the decomposition performance and the corresponding IMF spectra of the two methods are shown in Figure 3.3 and Figure 3.4, respectively. 


\section{EMD of the noisy ECG signal}

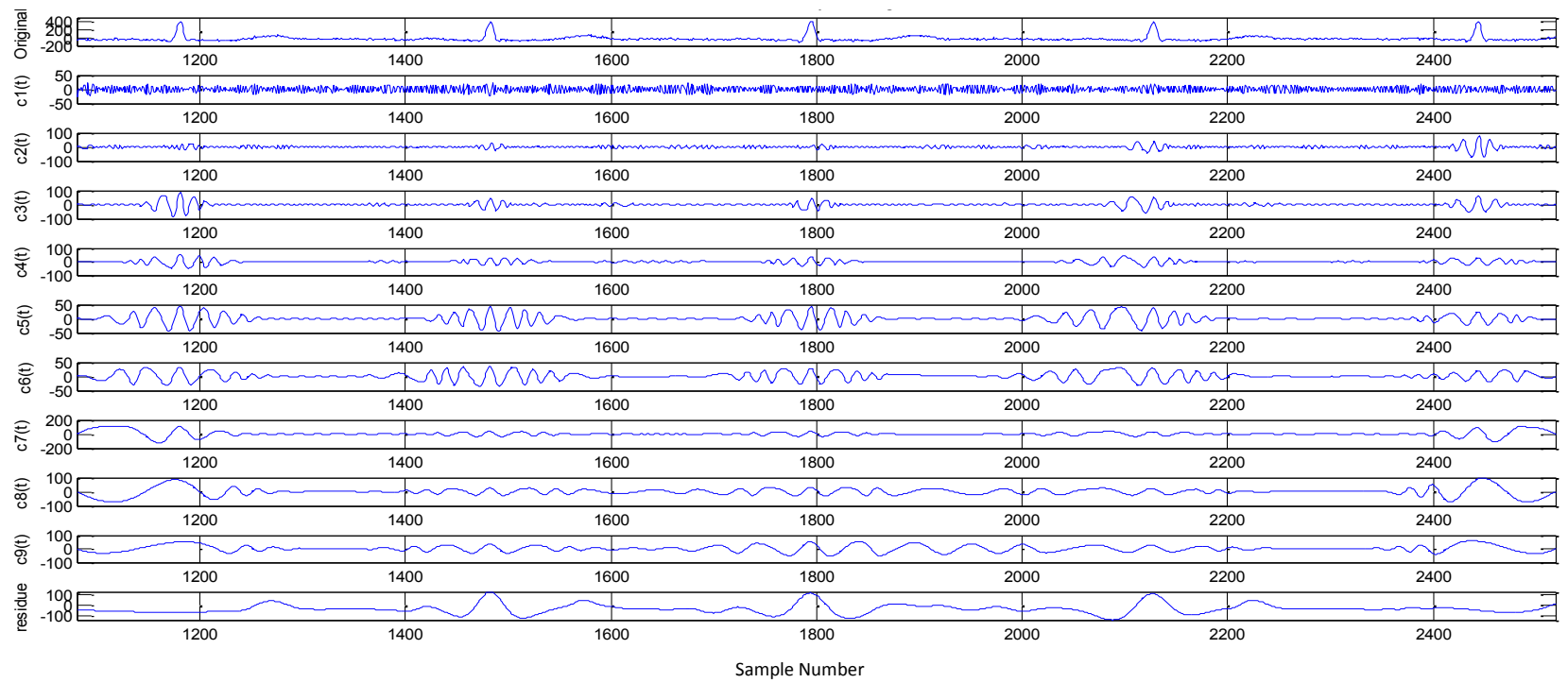

\section{EEMD of the noisy ECG signal}

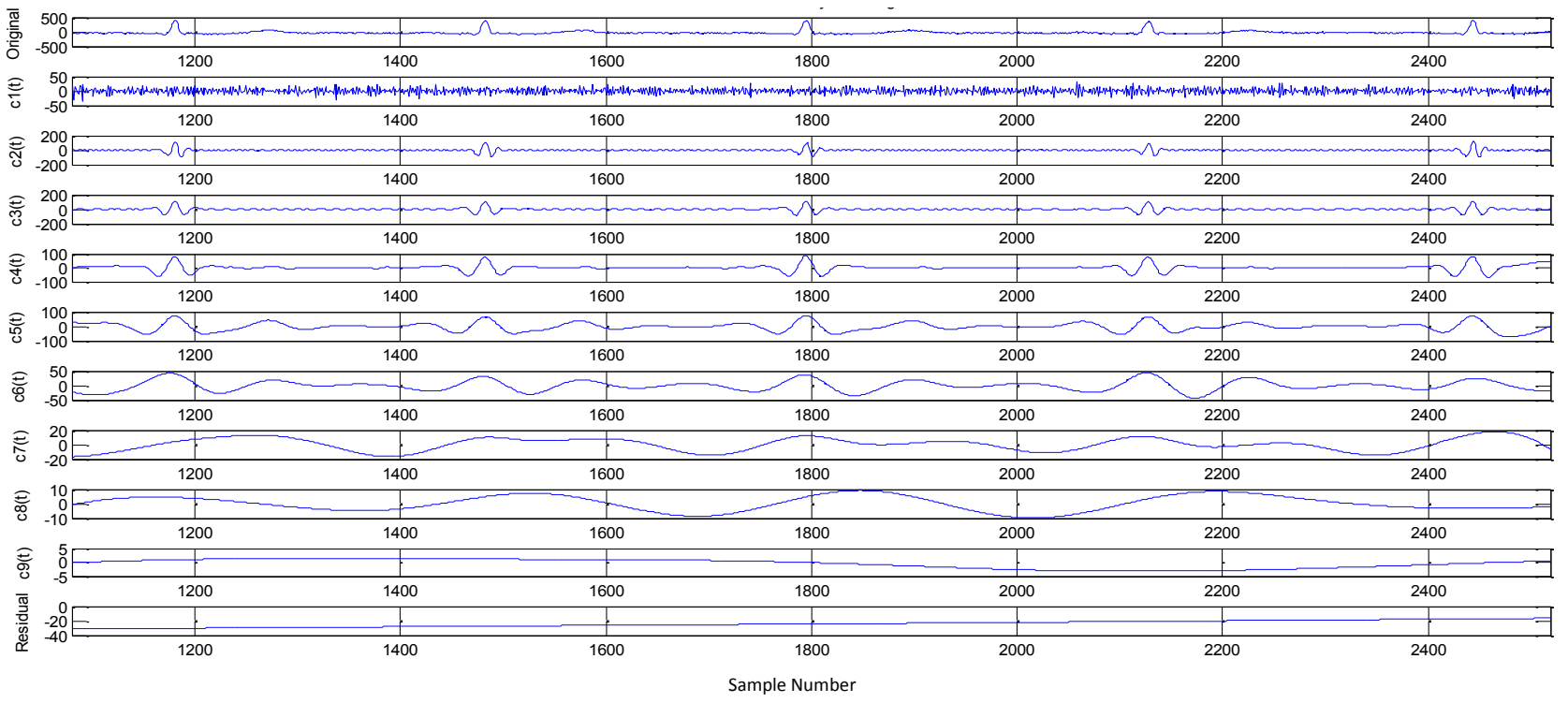

Figure 3.3: IMF distribution of EMD and EEMD for the noisy ECG signal. The noisy ECG signal is contaminated by Gaussian noise with the SNR value of $10 \mathrm{~dB}$ 
Single-Sided Power Spectra of the IMFs of EMD

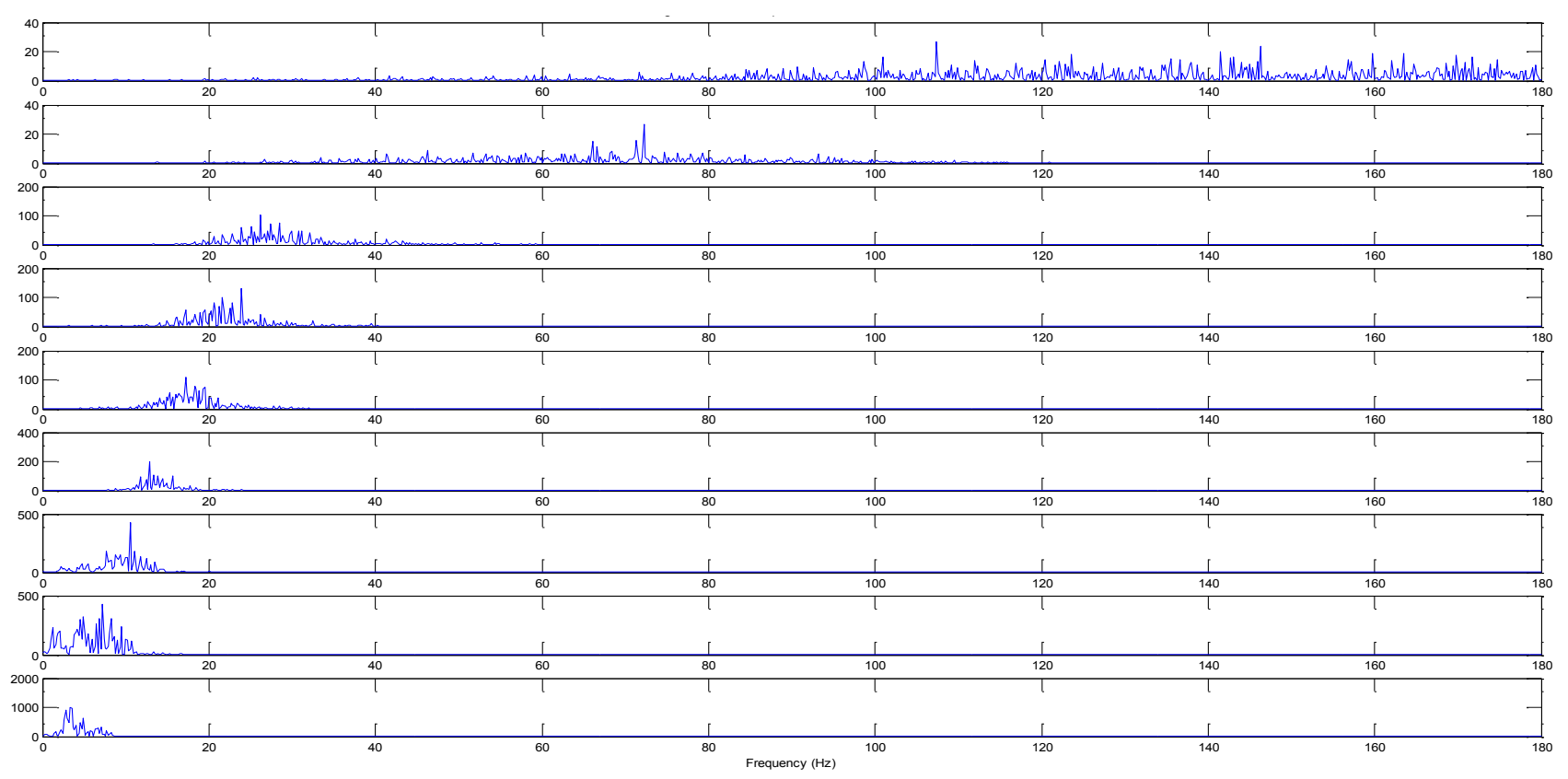

Single-Sided Power Spectra of the IMFs of EEMD

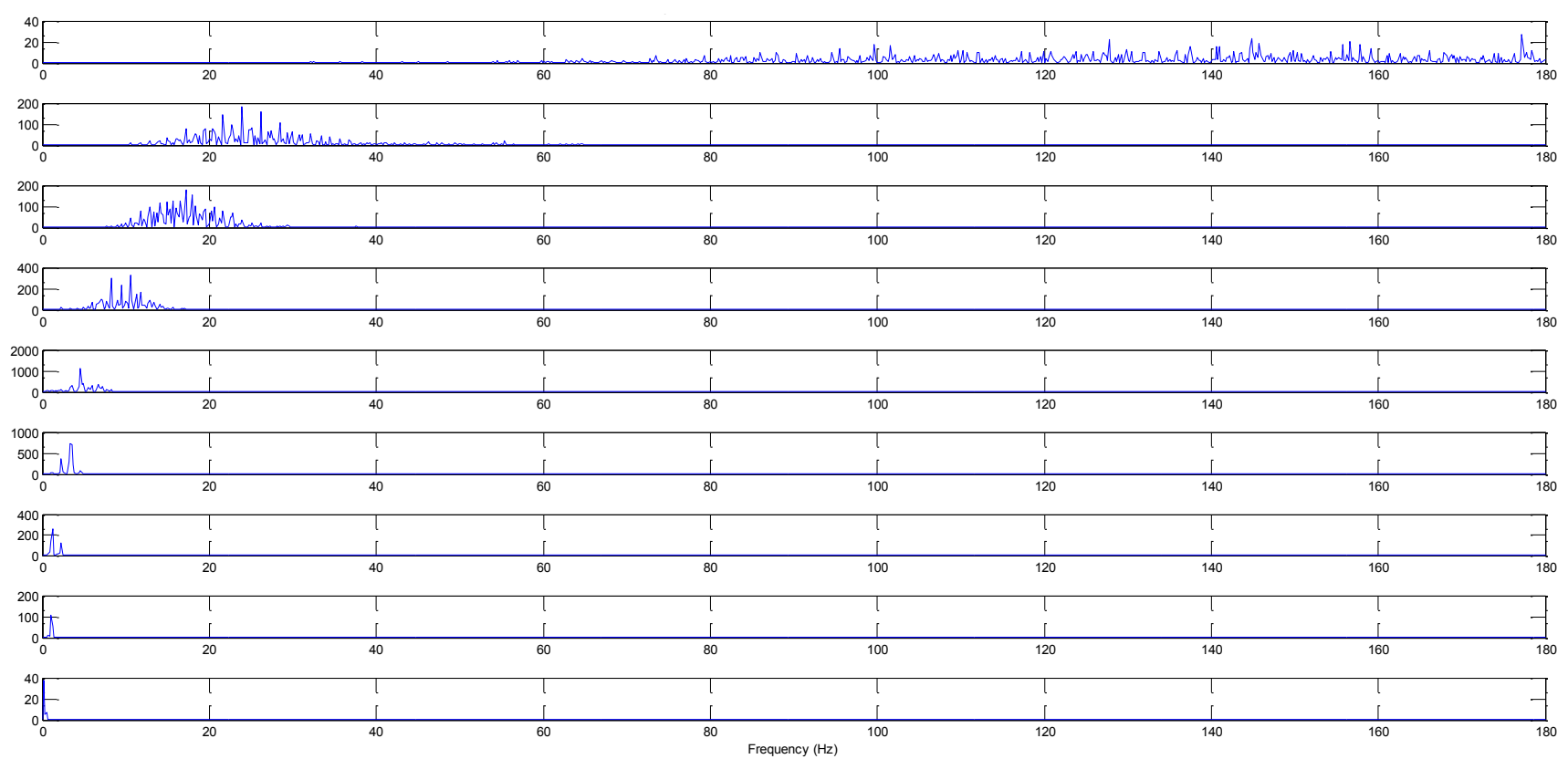

Figure 3.4: The power spectra of corresponding IMFs for the noisy ECG signal with $10 \mathrm{~dB}$

Gaussian noise 
From Figure 3.4, we can see that the high-frequency components are contained in the lower IMF scales, the low-frequency components are contained in the upper IMF scales, and the IMFs of EEMD are more concentrated and band limited. The power spectra can elaborate it much clearer that EEMD has better performance. There is an obvious spectrum overlapping between the IMF components of EMD at almost each scale, while the IMFs of EEMD are localizing in different ranges of frequency at each scale.

\subsubsection{Delineation of the QRS Complex}

In order to delineate the QRS complex, the low IMF scales are considered, because the QRS complex spreads over the lower-order IMFs based on the principle of the decomposition of the EMD/EEMD algorithm. In other words, although the lower-order IMFs correspond to some high-frequency noise, we cannot just remove them simply and reconstruct the original signal by summing up the remaining IMFs without the first several IMFs.

It is mentioned that the first several IMFs can be jointly used to delineate the QRS complex [56]. Only the EMD method is used in this part. In order to demonstrate the advantage of EEMD, we compare the delineation of the QRA complex in the EMD domain and the EEMD domain by adding the first four IMFs as shown in Figure 3.5 and Figure 3.6 , respectively.

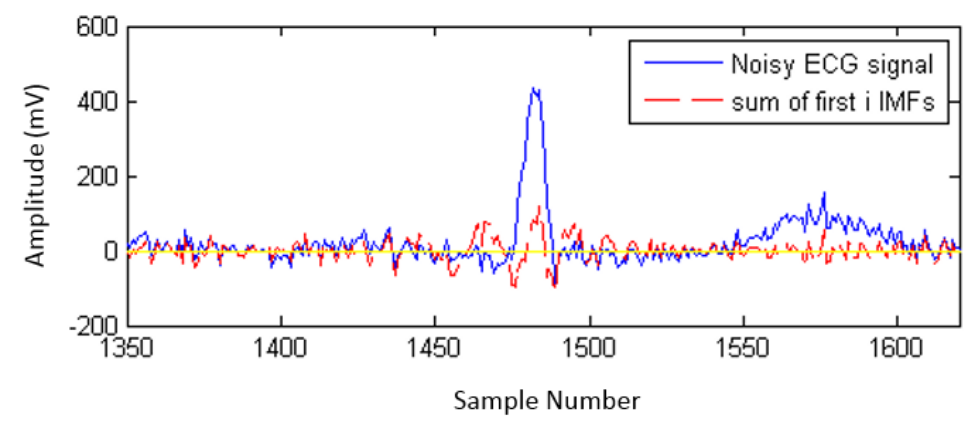

Figure 3.5: Delineation of the QRS complex in the EMD domain 


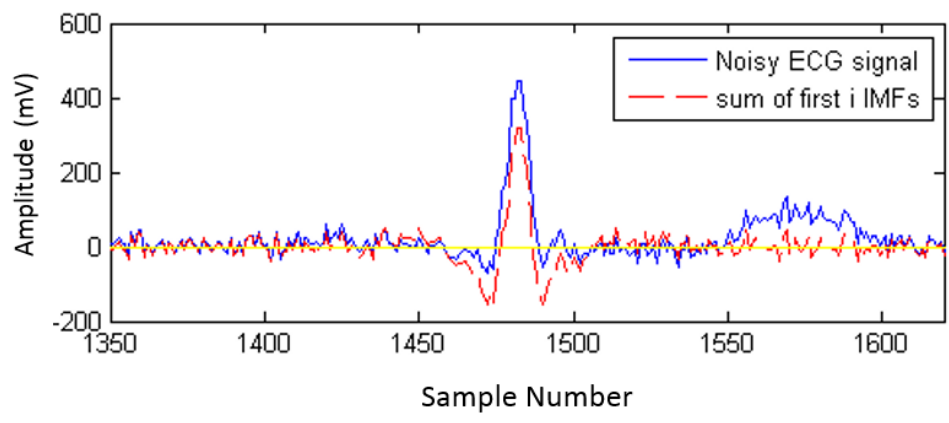

Figure 3.6: Delineation of the QRS complex in the EEMD domain

With the summation of the same number of IMFs, the EEMD algorithm has a better performance in terms of detecting the QRS complex, and the low IMF scales of EEMD contain more important QRS complex information.

After the summation of the first four IMFs is obtained, it still needs to identify the fiducial points and the boundary lines of the QRS complex to separate the QRS complex. The fiducial points can be determined by the curve of the signal or the annotation from the database.

For ECG signals contaminated by the contact noise, the method used to detect the two closest zero-crossing points described in [105] is no longer effective, because the contact noise contains some low-frequency noise or baseline wandering that changes the baseline of the ECG signal, and some segments of the signal may not cross the axis anymore. In this study, we develop a new method described as follows to detect the QRS complex. The steps are as follows:

Step 1) Identify the fiducial points of the noisy signal, which are the peaks of the signal; 
Step 2) Apply EEMD to the noisy signal, and sum up the first four IMFs to obtain $s(t)$

Step 3) Calculate the derivative of $s(t)$ and detect the difference of the sign of the nearest derivative on the both sides of the fiducial points. The two points when the sign changes twice are identified as the boundary lines of the QRS complex.

A segment of the detection result is shown in Figure 3.7. The QRS complex can be separated accurately from the noisy signal.

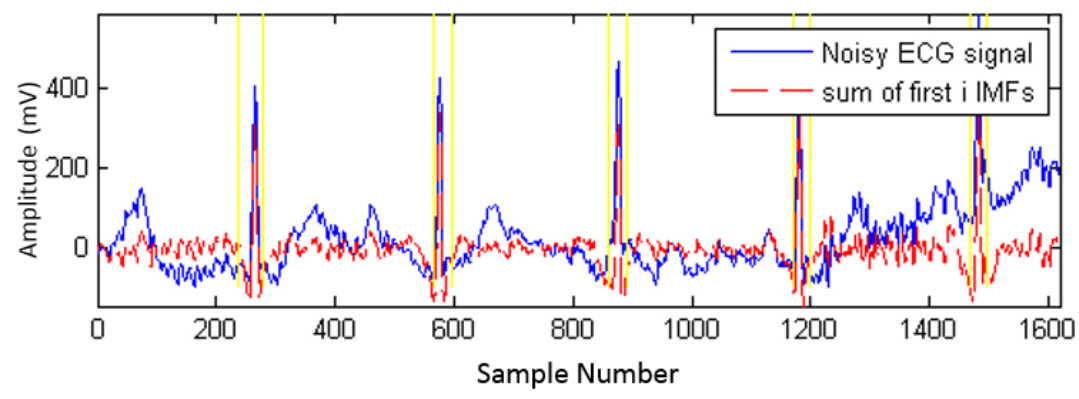

Figure 3.7: The boundary lines of the QRS complex. The yellow lines are the boundary lines. The noisy signal is the clean ECG signal contaminated by the contact noise with the SNR value of $10 \mathrm{~dB}$

\subsubsection{Preservation of the QRS Complex}

After the boundary lines of the QRS complex are known, windowing is used to preserve the QRS complex components exist in the first several IMFs. As said in [105], the requirements of the used window are flat out during the QRS complex segment, reduce gradually to zero, and its function should adjust the size according to the spreads of the QRS complex. We use Kaiser Window, because compared with the other windows, it has narrower main lobe and lower ripples, and it can be described as: 


$$
w[n]= \begin{cases}\frac{I_{0}\left[\beta \sqrt{1-\left((2 n-M) / M^{2}\right)}\right]}{I_{0}(\beta)}, & 0 \leq n \leq M \\ 0, & \text { otherwise }\end{cases}
$$

where $I_{0}$ is the 0th-order modified Bessel function of the first kind. $\beta$ determines the shape of the window. $M$ is the length of the window, which depends on the spreads of the QRS complex.
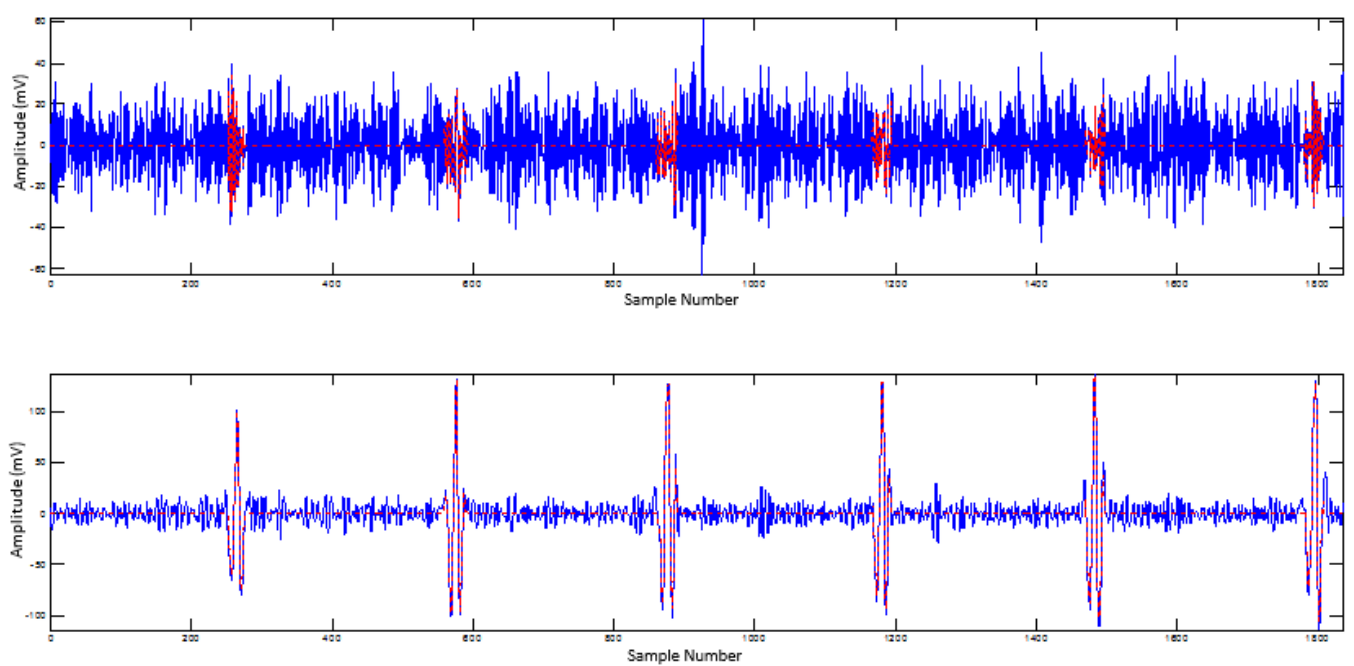

Figure 3.8: The first two IMFs after windowing

The first two IMFs of the Gaussian noisy signal are shown in Figure 3.8. The QRS complex components can be preserved and separated from the noisy signal.

During the preservation of the QRS complex, one more factor should be considered, that is the number of the noise-dominated IMFs. A statistical test named t-test is used in [105] and [154].

\subsubsection{Elimination of Low-frequency Noise}

The higher orders of IMFs contain the low-frequency components of the noise. The residue of the decomposition process should be also regarded as the last IMF, but it does 
correspond to the low-frequency noise, because it does not have multiple extrema and zerocrossings. Although the last several IMFs have significant noise components, the removal of the last several IMFs will cause serious distortion. There is also some important information about the original clean signal spreading over the last several IMFs. We use a series of high-pass filter $h_{i}(t)$ to eliminate the low-frequency noise.

\subsubsection{Partial Reconstruction}

Both low-frequency and high-frequency noise is considered in this study. Based on the elaboration above, the equation used to partially reconstruct the ECG signal can be written as:

$$
\begin{gathered}
\tilde{x}(t)=\sum_{i=1}^{H} w_{i}(t) c_{i}(t)+\sum_{i=1}^{H} \alpha_{i} \bar{w}_{i}(t) c_{i}(t) \\
+\sum_{i=H+1}^{N} c_{i}(t)+\sum_{j=1}^{L} \sum_{j=1}^{L} h_{j}(t){ }^{*} c_{N-j+2}(t)+r_{N}(t)
\end{gathered}
$$

There are five parts in the reconstructed signal $\tilde{x}(t)$. The first part is the windowed IMFs for delineating the QRS complex. $H$ and $L$ are the number of IMFs which contributes primarily to the high-frequency noise and low-frequency noise, respectively. $\bar{w}_{i}(t)$ in the second part is the conjunction of $w_{i}(t)$. The third part is the remaining order of IMFs. The following part is the filtered IMFs that contain low-frequency noise and the last item is the residue.

\subsubsection{Reference Input of the Adaptive Filter}

As for the input signals of the adaptive filter, the more convenient and easier way to obtain the reference signal is from the original noisy signal itself, because no more signal generator or extra leads are needed. In the meantime, the complexity of hardware and software can be reduced. 
The EEMD method could separate the original signal into a series of IMFs, which are distributing from local high frequency to local low frequency, as filter banks, but not band restricted. The decomposition processing is adaptive and is totally based on the pattern of the original signal. The contact noise can be selectively reconstructed by certain number of IMFs and the reconstructed signal can be used as the reference input of the adaptive filter. The reference input obtained from signal reconstruction after EEMD for the noisy signal with Gaussian white noise, muscle artifacts, and motion artifacts are shown in Figure 3.9, Figure 3.10, and Figure 3.11, respectively. All the SNR values of the noisy signals are $10 \mathrm{~dB}$.

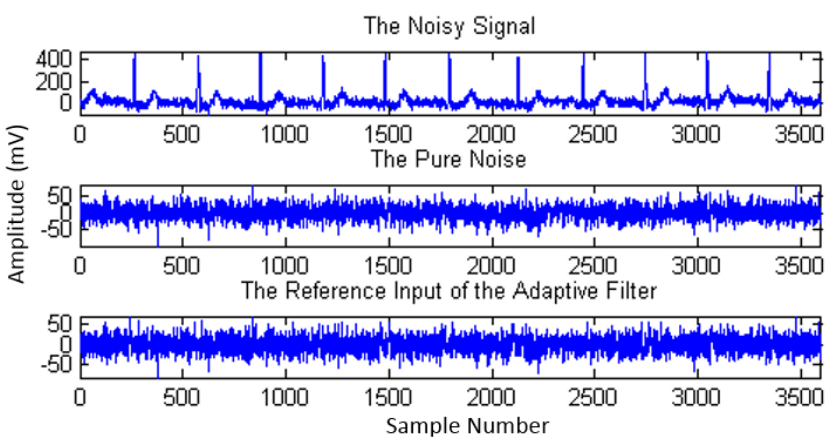

Figure 3.9: The noisy signal, pure Gaussian noise, and the reconstructed signal as the reference input of the adaptive filter

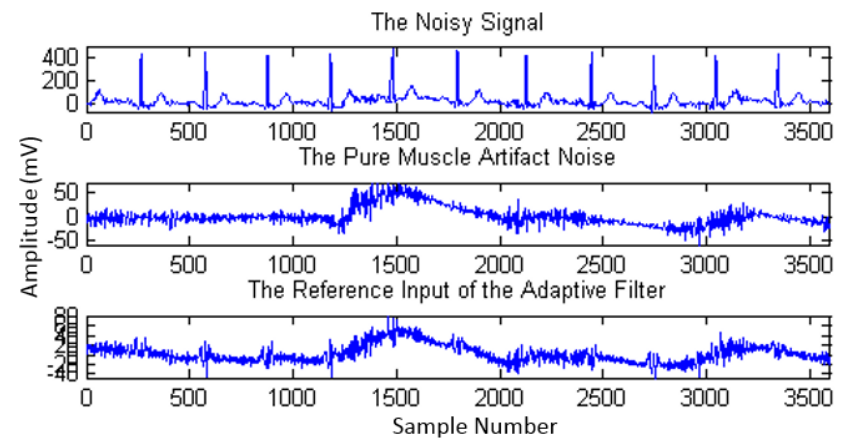

Figure 3.10: The noisy signal, pure muscle artifacts, and the reconstructed signal as the reference input of the adaptive filter 


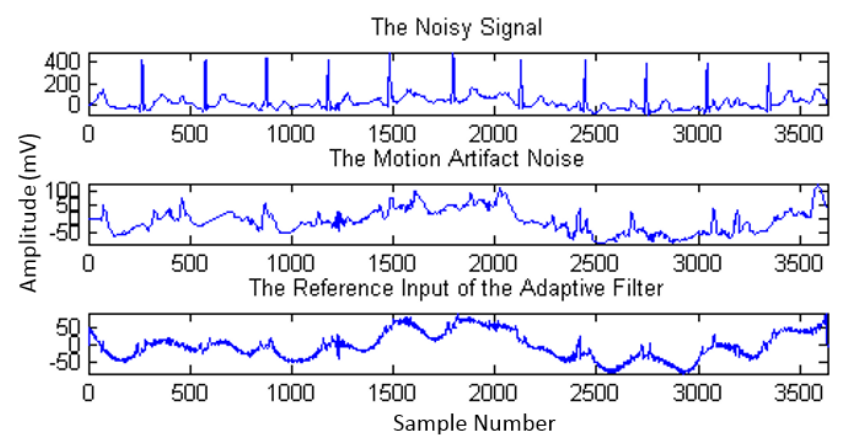

Figure 3.11: The noisy signal, pure motion artifacts, and the reconstructed signal as the reference input of the adaptive filter

The correlation coefficients between each type of reference input signal and the pure noise are $0.92,0.85$, and 0.85 , respectively. The values of the correlation coefficients are all above 0.8 , which means that correlation is very high.

After obtaining the reference input, the LMS algorithm is used to adaptively update the coefficients of the adaptive filter to minimize the MSE of the output of the whole system. Once the minimum MSE value is achieved, the output is the enhanced ECG signal.

\subsection{Experimental Results}

Two sets of experiments were performed in this study. The first one was to use the Gaussian white noise as a test experiment. Record 103 is used as an example to evaluate the performance of our proposed EEMDA algorithm. The other experiment was to add muscle artifacts and motion artifacts into the clean ECG signal. Five records with three different SNR values are carried out, and the results are compared with the previous work.

The quantitative evaluation criteria used are signal-error-ratio (SER) and the displacement between the clean ECG signal and the reconstructed signal. By using the 
displacement, we can see the differences step by step, rather than the global difference, which may conceal some of the bad de-noising points. The equation of SER is:

$$
S E R=\sum_{0}^{N-1} x^{2}(t) / \sum_{0}^{N-1}[x(t)-\tilde{x}(t)]^{2}
$$

where $x(t)$ is the original signal and $\tilde{x}(t)$ is the reconstructed signal.

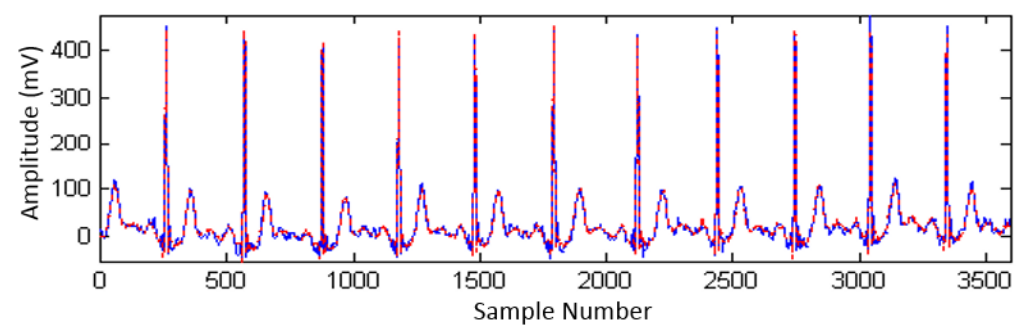

(a)

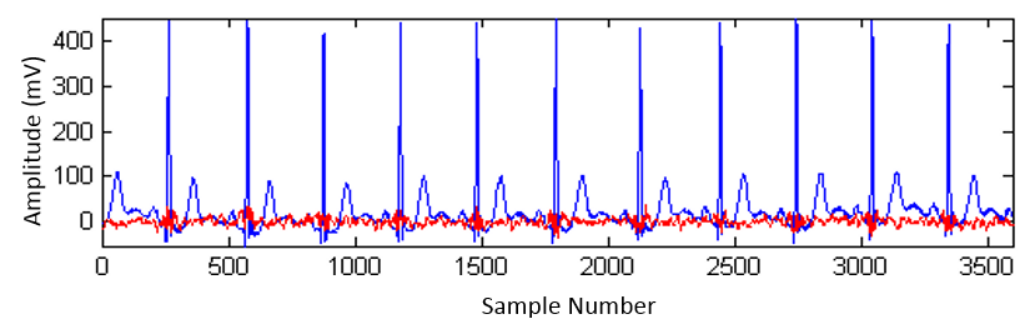

(b)

Figure 3.12: The denoising results of the Gaussian noisy signal with SNR value of $10 \mathrm{~dB}$

The clean ECG signal and the Gaussian noisy signal are shown in Figure 3.2. By using the EEMDA method, the result of the ECG de-noising for Gaussian noise is shown in Figure 3.12.

In Figure 3.12 (a), the red dashed graph stands for the clean ECG signal, and the blue solid graph stands for the reconstructed signal. In Figure 3.12 (b), the red line is the displacement. The maximum value of the displacement is $34.73 \mathrm{mV}$, and the minimum value is $5 \times 10^{-4} \mathrm{mV}$. The SER for the reconstructed signal is $18.93 \mathrm{~dB}$. In Blanco-Velasco's work, the SER is $18.85 \mathrm{~dB}$. The advantage of the proposed EEMDA method is not 
significant from the results of this experiment, but the superiority of the developed EEMD will be shown clearly in the next experiments for which the ECG signal is corrupted by real contact noise.

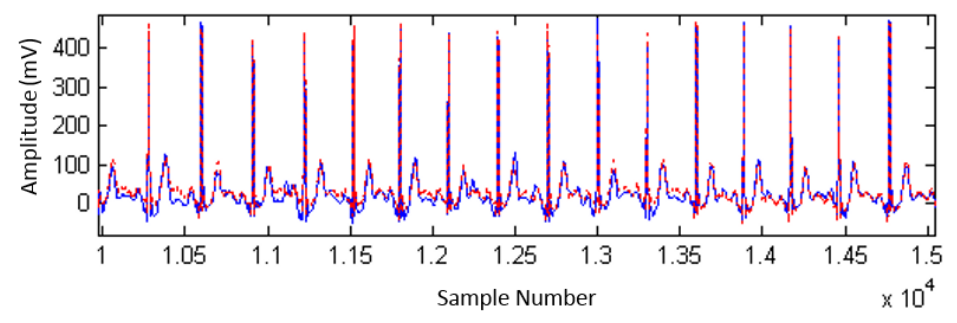

(a)

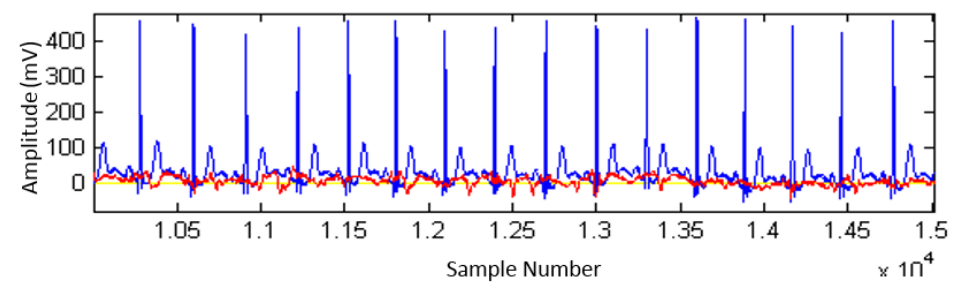

(b)

Figure 3.13: The EEMDA method de-noising results of the contact noisy signal with SNR value of $10 \mathrm{~dB}$

In order to compare the de-noising performance to previous work, long-term experiments were carried out. Records 100, 103, 105, 119, and 213 were used, and three different values of SNR were taken. Taking record 103 as an example, the reconstructed results and the displacement by using EEMDA method are shown in Figure 3.13.

Figure 3.13 (a) shows the comparison between the reconstructed signal (blue graph) and the original signal (red graph). We can see that the dominating components of the noise have been eliminated. Figure 3.13 (b) shows the displacement of the results with the maximum value of $43.00 \mathrm{mV}$ and the minimum value of $6.5 \times 10^{-3} \mathrm{mV}$, respectively. 
The results of applying the EEMDA method in this chapter are mainly compared with the results achieved by Blanco-Velasco [105] who studied the ECG de-noising problem by using EMD and compared the results with the Butterworth low-pass filtering and the wavelet-based method. The comparisons are presented in Table 3.1. Due to the usage of the adaptive filtering, the performance of the EEMDA method is better than the performance of the EMD method.

Table 3.1: The comparison of the de-noised results of the EEMDA method and the EMD-based method for the first 46000 samples of selected records

\begin{tabular}{ccccccc}
\hline \multirow{2}{*}{ Record } & \multicolumn{2}{c}{ SNR $=\mathbf{6 ~ d B}$} & \multicolumn{2}{c}{ SNR $=10 \mathbf{d B}$} & \multicolumn{2}{c}{ SNR = 14 dB } \\
& SEREEMda & SEREMd & SEREemda & SEREMd & SEREEMda & SEREMd \\
\hline 100 & 11.57 & 11.40 & 15.53 & 13.95 & 17.39 & 16.75 \\
103 & 11.38 & 9.85 & 14.65 & 12.90 & 18.57 & 15.70 \\
105 & 12.70 & 9.62 & 15.96 & 11.94 & 18.68 & 14.54 \\
119 & 15.49 & 11.45 & 20.68 & 14.71 & 22.02 & 17.29 \\
213 & 9.15 & 8.87 & 15.24 & 11.89 & 17.99 & 14.74 \\
\hline
\end{tabular}

Taking record 103 with contact noise as an example, the reconstructed results and the displacement by using EEMDA method are shown in Figure 3.14. The value of the step size was 0.1 and the filter order used was 25 .

In Figure 3.14, the blue graph is the filtered signal and the red graph is the noisy signal. The value of SER is 14.97, and the maximum and minimum displacement is 44.01 $\mathrm{mV}$ and $6 \times 10^{-4} \mathrm{mV}$, respectively. The results demonstrate that the EEMDA method has a 
good performance in reducing the contact noise in ECG signals and the characteristics of ECG signals is kept after the de-noising processing.

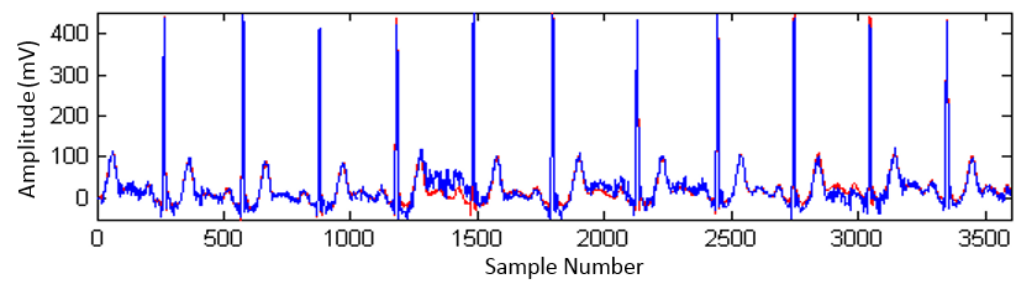

(a)

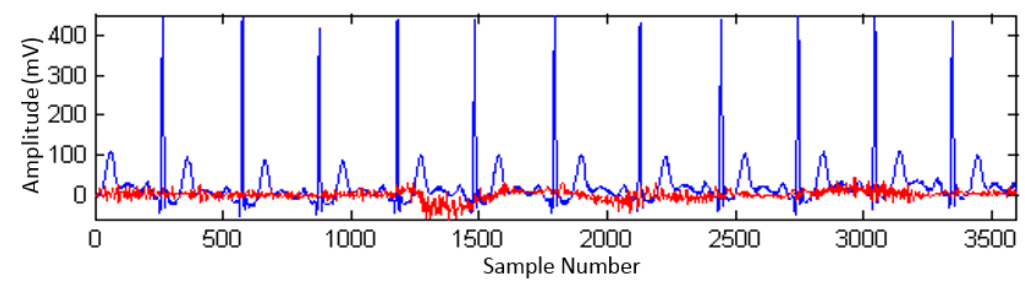

(b)

Figure 3.14: The EEMDA method de-noising results of the contact noisy signal with SNR value of $10 \mathrm{~dB}$

To better show the performance of the proposed EEMDA algorithm, an interface was designed by MATLAB Graphic User Interface (GUI), shown in Figure 3.15. The functions of the interface included importing original ECG signals from the MIT-BIH database, plotting the original and noisy data in time and frequency domain, adding Gaussian noise to the original ECG signal with adjustable SNR, applying the EEMD and plotting the single-sided power spectrum of each IMF, applying the EEMDA algorithm and displaying the de-noised ECG signal, giving the SER value and saving the results. The "Zoom In" and "Zoom Out" buttons were designed for observing a specific area on each plot. 


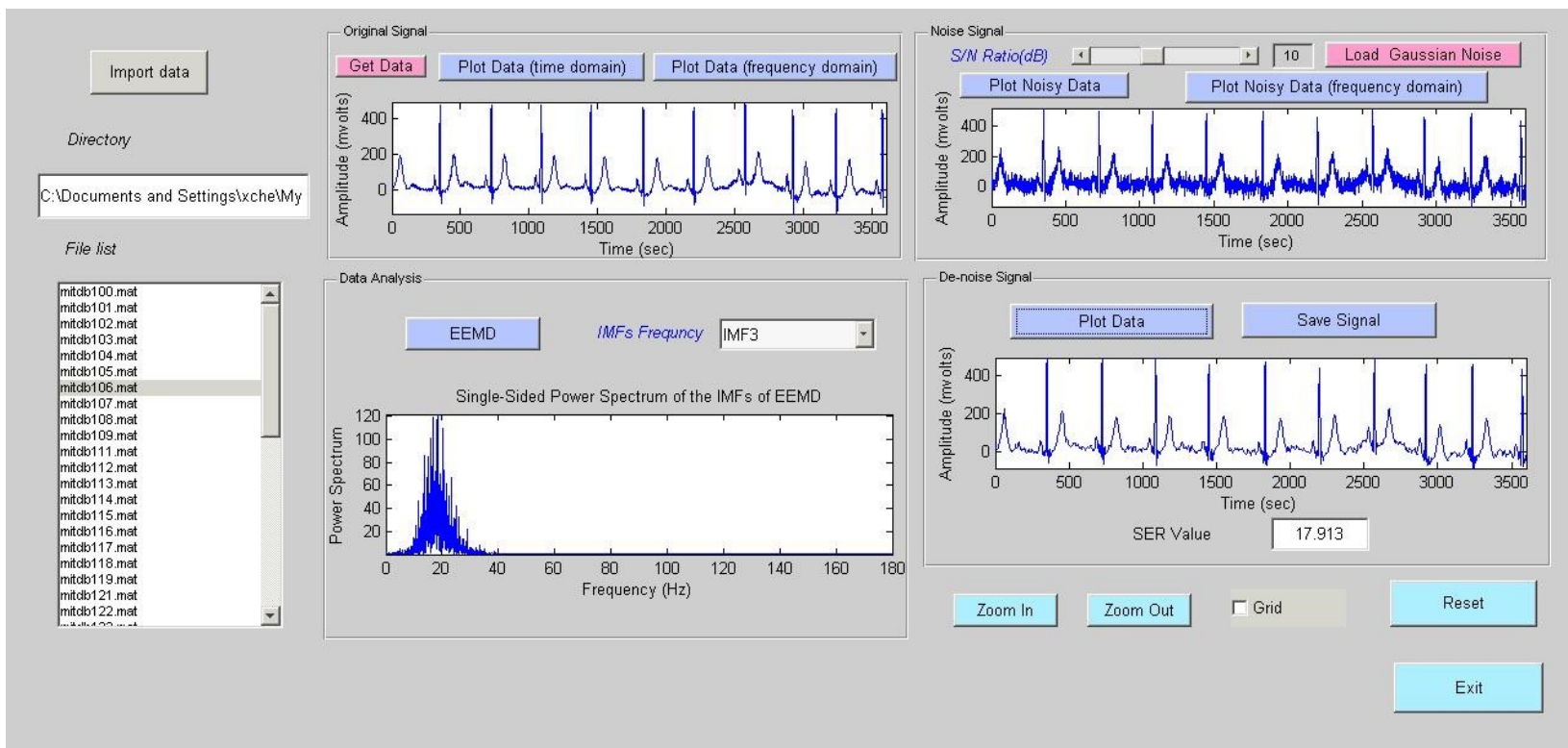

Figure 3.15: Interface designed by MATLAB GUI

\subsection{Summary and Conclusion}

This chapter presented a novel signal enhancement method based on EEMD and adaptive filtering to remove noise from corrupted ECG signals while keeping all the ECG features. Two types of noise were considered: the Gaussian white noise and the contact noise. The new EEMDA method, even by using the basic LMS algorithm, had better performance than the EMD and EEMD method. Another advantage of the developed EEMDA method was that its de-noising processing was less complicated because the delineation and reservation of the QRS complex, the t-test to confirm the number of IMFs that were dominated by noise were not required to be taken into consideration. Experiments on real ECG signals corrupted by noise showed that the developed method can enhance the ECG signal significantly. 
Moreover, the EMD-based sub-band adaptive filtering structure was proposed. This structure was assessed for the application of acoustic echo cancellation. Appendix A presents the results associated with the use of the EMD-based sub-band adaptive filtering structure in acoustic echo cancellation. The basic principle of the structure was to separate the far-end speech signal and the microphone output signal into different sub-band based on the power spectral density of the signal itself. The simulation results had shown the efficiency of the proposed structure and proved that the EMD-based algorithm could be applied to solve echo cancellation problems, and the EEMD and NEMD algorithms outperformed the EMD algorithm. 


\section{Chapter 4}

\section{PPG Signal Description, Modeling, and Peak Detection}

As indicated in chapter 3 , it is possible to estimate the arterial blood pressure from the pulse transit time (PTT). One possible method of estimating the PTT is to calculate the delay between the peak of the photoplethysmograph (PPG) signal from a finger and the R-peak of the electrocardiogram (ECG) signal. However, noise on the PPG and ECG signals leads to inaccuracies in estimating these peaks. The objective of this chapter is to propose approaches to accurately detect the peak locations of the PPG signal.

Figure 4.1 illustrates the characteristic parameters employed for blood pressure estimation. The red curve, blue curve, and green curve represent the blood pressure, finger PPG signal, and ECG signal, respectively. The R-peak index is determined by locating the local maxima of each ECG cycle. The systolic blood pressure (SBP) is calculated based on the maximum points of the blood pressure. The diastolic blood pressure (DBP) is calculated based on the minimum points of the blood pressure. The dicrotic notch of the PPG signal is visible in Figure 4.1. But not all the dicrotic notch of the PPG signal is visible from the MIMICII database. 


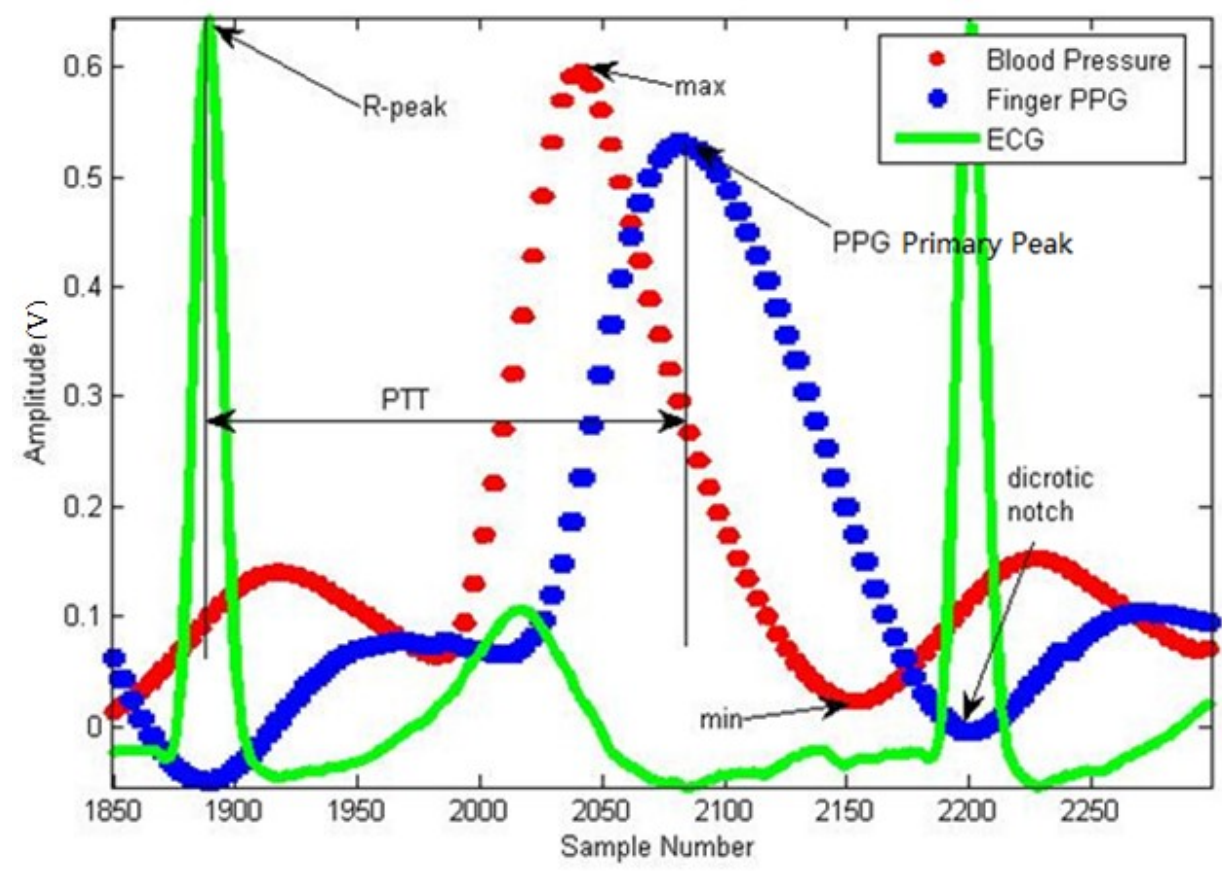

Figure 4.1: Characteristic parameters and PTT determined from one set of synchronous ECG and finger PPG signals used for blood pressure estimation

\subsection{PPG Signal Description}

It has been widely demonstrated in the literature that the PPG signal can be used to monitor many physiological parameters, such as blood oxygen saturation $(\mathrm{SaO} 2)$, arterial blood pressure, respiration, heart rate (HR), and cardiac output (CO) [130]. PPG signal has also been utilized as a noninvasive method in vascular assessment and autonomic function assessment, such as arterial stiffness, aging, thermoregulation, blood pressure, and heart rate variability [130]. The full understanding of the hidden physiological information in the PPG signal is still a hot topic [165]. 


\subsubsection{Ideal PPG Signal Waveform}

The PPG signal usually has a primary peak and may show additional secondary peaks. Figure 4.2 shows an ideal PPG signal. Generally, the PPG signal can be regarded as containing two phases: the anacrotic phase and the catacrotic phase. The anacrotic phase is mainly concerned with systole, while the catacrotic phase is about the diastole and the wave reflections from the periphery [166]. Figure 4.2 also illustrates some of the characteristic parameters of the PPG signal in one cycle. The primary peak is the location of the maximum value of the PPG signal. The secondary peak is the location of the second peak. The dicrotic notch is the valley between the primary peak and the secondary peak. $A 1$ is the amplitude of the primary peak and $A 2$ is the amplitude of the secondary peak. $T 1$ is the crest time. $T 2$ is the secondary wave time, and $T 3$ is the pulse duration.

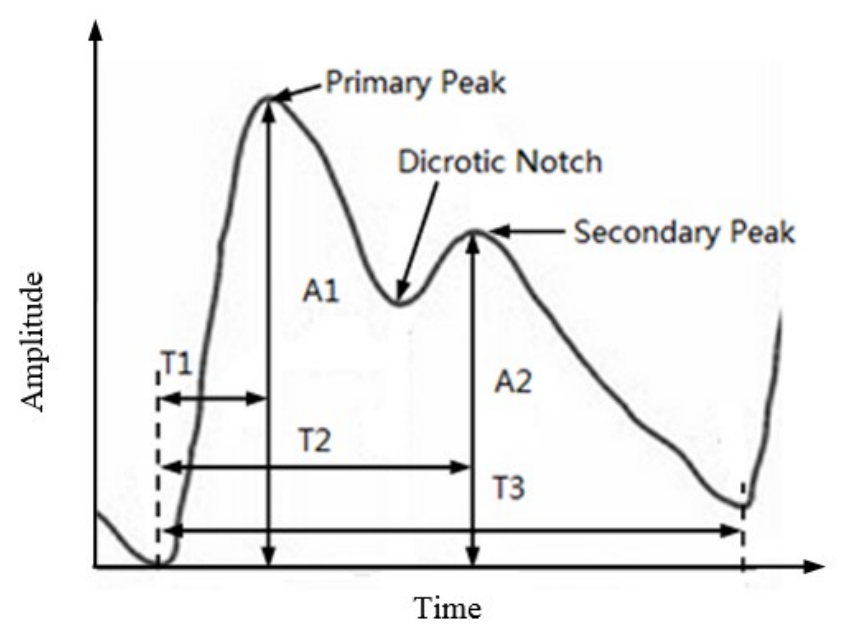

Figure 4.2: Ideal PPG signal and its characteristic parameters 


\subsubsection{Clinical PPG Signal and Challenges}

The dicrotic notch of the PPG signal is usually visible only in the catacrotic phase of subjects with a healthy cardiovascular status [167]. Sometimes the secondary peak is embedded in the whole PPG signal, especially when the subjects are old people or people with cardiovascular disease. Figure 4.3 is an example of the clinical PPG signal with a negligible dicrotic notch. To distinguish the peak in Figure 4.3 from the primary peak shown in Figure 4.2, this only peak of the clinical PPG signal is defined as main peak.

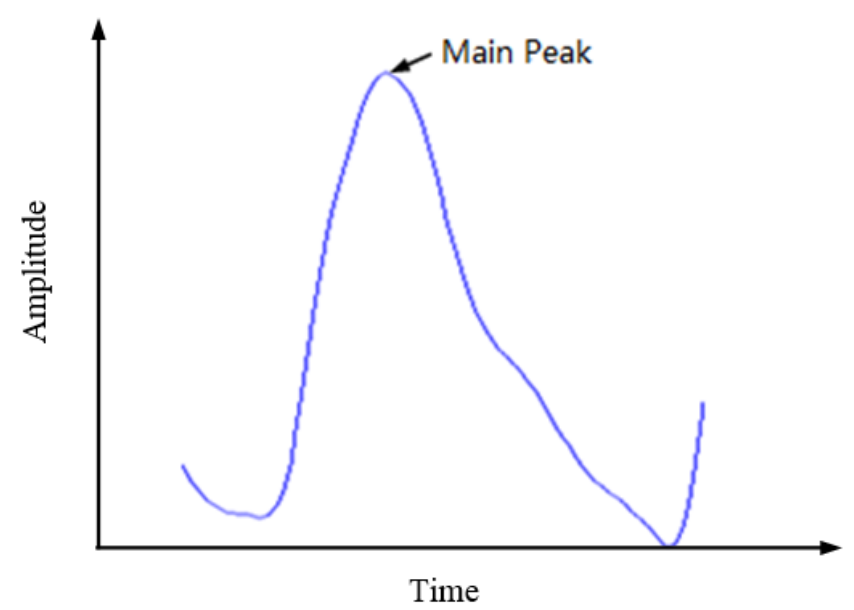

Figure 4.3: Clinical PPG signal without visible dicrotic notch

As the disappearance of the dicrotic notch, the location and the amplitude of the maximum value of the PPG signal will be changed from the primary peak to the main peak. This effect will result in the disappearance of certain characteristic parameters of the PPG signals and lead to inaccurate measurement of PTT. As a result, the correlation between the blood pressure and the PTT can be affected [167]. To overcome the above problems, the detection of the primary peak of the PPG signal has become an essential procedure to 
improve the accuracy of the measurement of PTT. The detection of the primary peak is also the detection of the secondary peak and the dicrotic notch.

\subsection{PPG Signal Modeling}

Generally speaking, instead of providing a methodology for modeling the PPG signal, former researchers mainly focus on analyzing the PPG signal [168]. A nonlinear signal model was proposed to analyze and handle the nonlinearity of the PPG signals in [169]. Three different sinusoidal source signals were applied to reproduce the primary harmonic, the second harmonic, as well as the respiratory and other spectra. The performance of the nonlinear model was evaluated on the subjects of smoking, drinking, and taking physical exercise. As the nonlinearity of the PPG signal got stronger, the reproduction ability of the model became weaker.

In [170] selected parametric models were developed to fit the PPG signal. The high frequency components of the PPG signal were selected, since they contained valuable information about the arterial blood pressure. Two points, the left and the right index fingers, were simultaneously measured. Four linear parametric models, Auto-Regressive with eXogenous input (ARX), Auto-Regressive Moving Average with eXogenous input (ARMAX), Output Error (OE), and Box-Jenkins (BJ) were proposed. The performance of four linear parametric models with different polynomial values was compared. Due to the linearity limitation, the four models were not suitable when the period of the PPG signal was long.

Given the nature of the PPG signal, Gaussian curves were employed to model the PPG signal in [168][171][172]. Regarding the PPG signal was formed by a forward wave 
and a backward wave, a model consisting of two Gaussian curves was used and a linear local trend was considered for a better fit. In mathematical view, each PPG cycle could be summarized by a parameter vector containing ten variables. Since the PPG signal is patientspecific, statistical atlases were also constructed in [171].

Traditionally, the contour of the PPG signal is described as a combination of four waves, including one primary wave and three reflected waves [171][172]. After dividing the PPG signal into two phases, the systolic phase (anacrotic phase) and the diastolic phase (catacrotic phase), each phase of the signal was fitted by the summation of two Gaussian curves. That is to say, four Gaussian curves in total were used to model the PPG signal.

\subsubsection{Gaussian Curves Modeling}

In this part, we applied two types of models: the two-Gaussian-curve model and the fourGaussian-curve model. After setting the initial values of the coefficients of the Gaussian curves, the fitted model was determined by the combination of two methods: the nonlinear least squares algorithm and the "Trust-Region" fitting algorithm. The equation used for describing the fitted model is as follows:

$$
\mathrm{G}(\mathrm{x})=\sum_{\mathrm{j}=1}^{\mathrm{n}} \mathrm{A}_{\mathrm{j}} \exp \left(-\frac{\left(x-\mu_{j}\right)^{2}}{2 \sigma_{j}^{2}}\right)
$$

where $A j$ is the amplitude, $\mu j$ is the center of the fitted curve, and $\sigma j$ is the width of the $j^{\text {th }}$ Gaussian curve. $x$ is the PPG signal sample. The relative sum of the least squares is set at $1 \times \mathrm{e}^{-6}$. When applying the two-Gaussian-curve modeling, $n$ is equal to 2 . And when using the four-Gaussian-curve modeling, $n$ is set to 4 .

Figure 4.4 and Figure 4.5 are examples showing the performance of the twoGaussian-curve model and the four-Gaussian-curve model, respectively. In both figures, 
the black curve is the original PPG signal. The red star curve stands for the fitted curve. In Figure 4.4, the blue and green curves represent the two modeling Gaussian curves, respectively. In Figure 4.5, the four Gaussian curves are represented by the blue, green, red, and cyan curves, respectively. The absolute differences between the original PPG and the fitted curves are $0.03 \mathrm{~V}$ and $0.007 \mathrm{~V}$ for two-Gaussian-curve modeling and four-Gaussiancurve modeling, respectively.

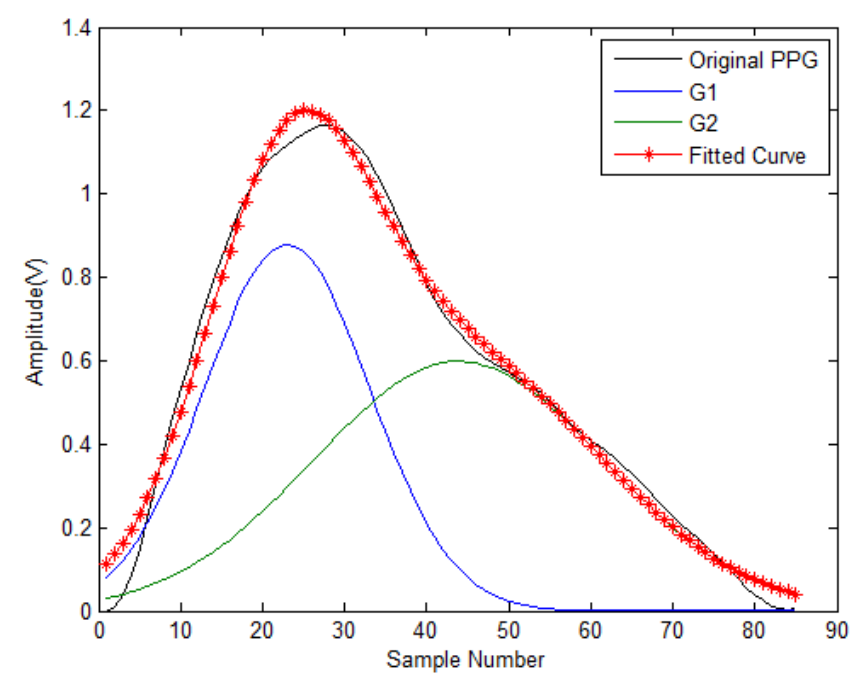

Figure 4.4: Two-Gaussian-curve model for PPG signal

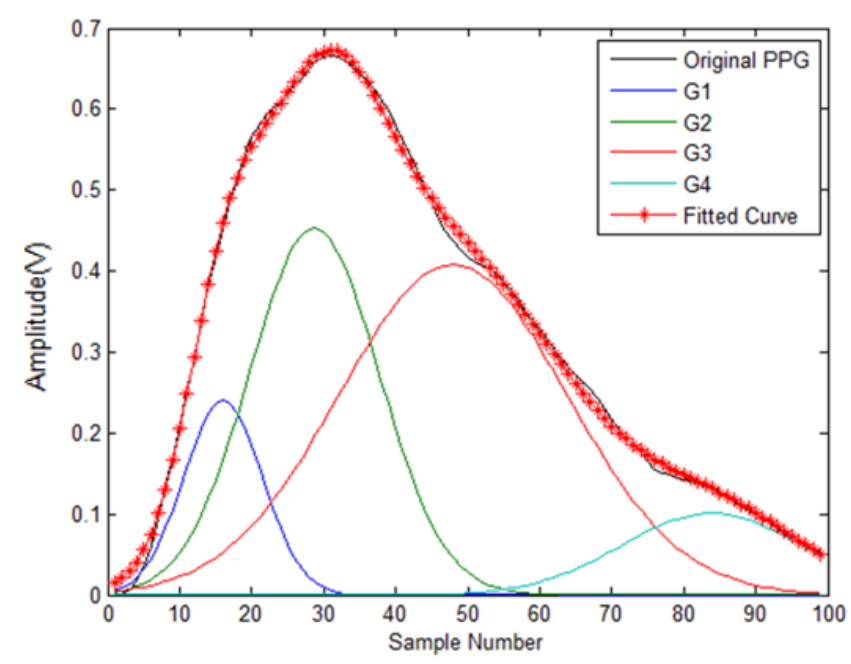

Figure 4.5: Four-Gaussian-curve model for PPG signal 
Figure 4.6 is the boxplot showing the comparison of the absolute differences between the original PPG signal and the two proposed models. It is obvious that the four-Gaussiancurve modeling has absolute advantage in fitting the original PPG signal with much smaller absolute difference value. The possibility of using the four-Gaussian-curve model to localize the primary peak will be discussed in section 4.7 .

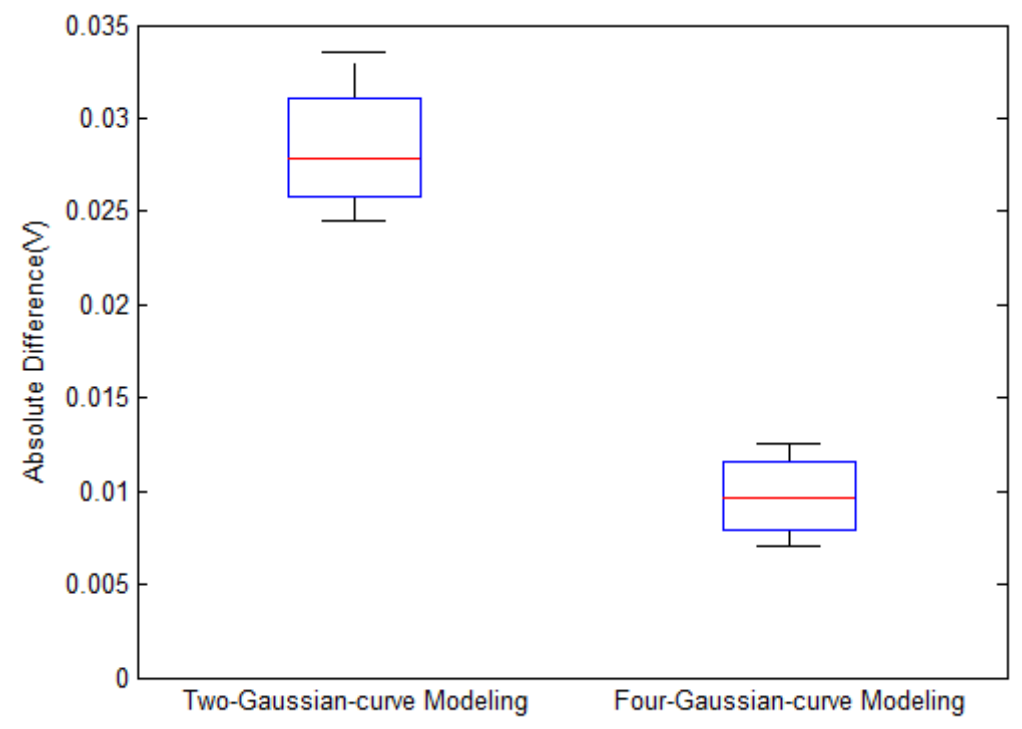

Figure 4.6: Absolute differences between the original PPG signal and the fitted curves by using two-Gaussian-curve modeling and four-Gaussian-curve modeling

\subsection{Signal Processing Methodology}

The primary peak of the PPG signal happens when blood is pumped from the heart and the secondary peak of the PPG signal is caused by the reflection of the blood in the vessel. When the reflection is not strong enough, the secondary peak will be buried and the dicrotic notch will become unobservable. The three method types to reveal the secondary peak of the PPG signal proposed in this chapter are: symmetrical curve fitting method (SCF), Gaussian curve fitting method (GCF), and adaptive curve fitting method (ACF). 
As mentioned in paper [24], the processing procedure of PPG peak detection involves several steps and can be described as follows:

Step 1) Select the relatively clean and complete signal segments that have the blood pressure in the designated range. The selected PPG records are regarded as the original PPG signals.

Step 2) De-noise the extracted signal segments.

Step 3) If the dicrotic notch of the original PPG signal is obvious, continue to step 4. If there is only one visible main peak of the PPG signal, apply secondary peak detection methods on the original PPG signal to visualize the secondary peak and relocate the primary peak of the PPG signal. The processed PPG signal is defined as the adjusted PPG signal. The procedure of the three different detection methods will be described in the following part.

Step 4) Detect the peaks of R-wave of the ECG signal and the peaks of the original PPG signal.

Step 5) Detect the maximum and minimum extremes of the blood pressure signal.

Step 6) Determine the delay between each corresponding R-peak and the adjusted PPG peak, which is the adjusted PTT.

Step 7) Calculate the correlation between the SBP and the adjusted PTT for each record and draw the linear, the quadric, and the cubic regression curves.

Step 8) Compare the fitted regression curves and select the best one for future blood pressure estimation. 


\subsubsection{Symmetrical Curve Fitting Method}

The principle of the symmetrical curve fitting method is to take the upstroke of the primary peak as the reference and reserve the primary peak of the original PPG signa. Then observe the secondary peak. The steps of the symmetrical curve fitting method are as follows:

Step 1) The upstroke side of the main peak of the original PPG signal mainly depends on the heartbeat. Select this part of the original PPG signal firstly as the reference $r$. Step 2) Find the axial symmetry of the reference $r$ curve regarded as $r \_s y m$. Integrate $r$ and $r_{-}$sym together to form the first peak signal noted as $p 1$, which is the primary peak. Pad $p 1$ to the same length as the original PPG signal.

Step 3) Subtract the padded $p 1$ from the original PPG signal and obtain the residue $p 2$, which is the secondary peak of the PPG signal.

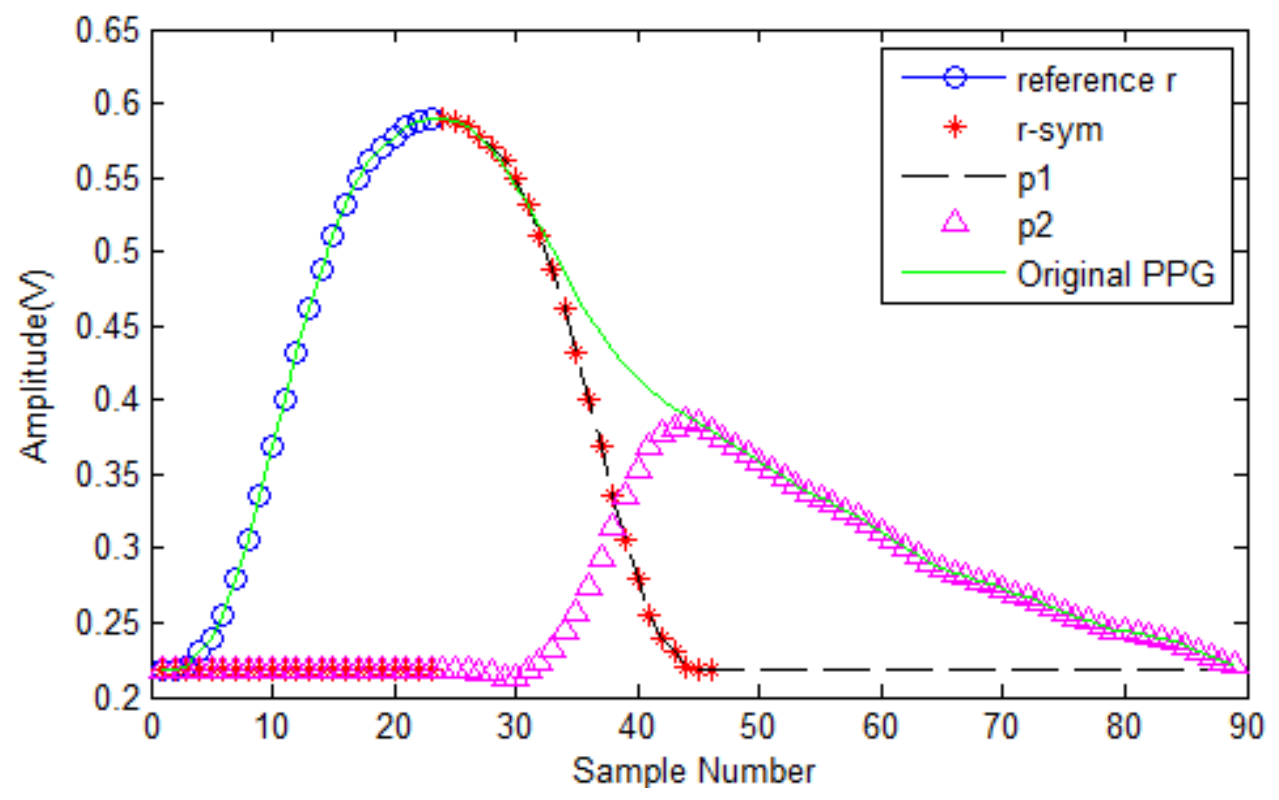

Figure 4.7: Illustration of the symmetrical curve fitting method 
Figure 4.7 illustrates the procedure steps of the SCF method. The blue circle curve represents the reference curve $r$. The red asterisk line is the axial symmetry of $r$. The black dash and the pink triangular line stand for the primary peak $p 1$ and the secondary peak $p 2$, respectively.

The position of the primary peak of the adjusted PPG signal is the same as the main peak of the original PPG signal. Since the SCF method is the first method we proposed to detect the secondary peak, the effect of the dicrotic notch to the PTT has not been considered. The simulation results in section 4.5 will show the improvement of the correlation between the blood pressure and the adjusted PTT with the concern of the peak shifting. This indicates the importance of the visibility of the secondary peak of the PPG signal in the blood pressure estimation.

\subsubsection{Gaussian Curve Fitting Method}

GCF method is another way to separate the secondary peak from the original PPG signal. Suppose the baseline of the fitting curve is at $g_{0}$ and the curve is not tilted. The Gaussian equation used only involves three parameters: the amplitude $A$, the mean $\mu$, and the standard deviation $\sigma$ [33]. The equation is normally expressed as:

$$
G=g_{0}+A e^{-(x-\mu)^{2}} / 2 \sigma^{2}
$$

One of the most important steps in applying GCF is to find the three parameters $A, \mu$, and $\sigma$ of the Gaussian curve that can best fit the upstroke side of the original PPG signal. $\mu$ is the center of the fitted curve and $\sigma$ controls the width of the curve.

The procedure of the GCF method is as follows: 
Step 1) Obtain the original PPG signal from the MIMICII database and look into the shape of the signal. If no dicrotic notch can be detected, GCF method needs to be employed.

Step 2) Calculate the second derivative of the original PPG signal, plot it, and observe the zero crossing points of the second derivative curve.

Step 3) Assume the zero crossing points around the main peak of the original PPG signal are $x_{1}$ and $x_{2}$, as shown in Figure 4.8. The position and the standard deviation of the fitted Gaussian curve can be estimated by using the following equations: $\mu=\left(x_{1}+x_{2}\right) / 2$ and $\sigma=\left|x_{1}-x_{2}\right| / 2$. The amplitude of the Gaussian curve $A$ is calibrated by the amplitude of the main wave of the original PPG signal.

Step 4) Subtract the Gaussian curve from the original PPG signal and get the secondary peak.

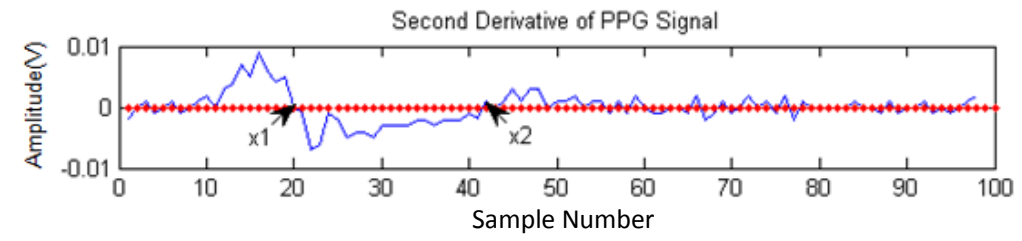

Figure 4.8: Second derivative of the original PPG signal and the zero-crossing points

Basically, the principle of the GCF method is to find the Gaussian curve that best fits the upstroke side of the original PPG signal. By finding the best fitted Gaussian curve, it means to locate the mean and the standard deviation of the fitted curve. Instead of using exhaustion method from observation, we decide to use the zero-crossing points of the second derivative curve to reduce the search range and the calculation complexity. 


\subsubsection{Adaptive Curve Fitting Method}

Based on the performance of the SCF method and the GCF method, an adaptive Gaussian curve fitting method is designed to detect the secondary peak from the original PPG signal. The fitting curve of the ACF method is a Gaussian curve and the procedure of the adaptive method is as follows:

Step 1) Obtain the original PPG signal and look into the shape of the signal. If no dicrotic notch can be detected, adaptive Gaussian curve fitting method needs to be employed.

Step 2) Adapt the mean and the standard deviation values based on the position of the main peak and the shape of the upstroke of the main peak.

Step 3) Adjust the amplitude of the fitting curve $A$ according to the amplitude of the main peak of the original PPG signal.

Step 4) Subtract the fitted curve from the original PPG signal to get the secondary peak of the adjusted PPG signal.

\subsubsection{Correlation}

In order to evaluate the strength and the direction of the relationship between the arterial blood pressure and the adjusted PTT signal, the correlation $r$ and $R^{2}$ are applied. The adjusted PTT is decreasing with the increasing of the blood pressure. The formula to calculate the correlation $r$ between the arterial blood pressure and the adjusted PTT is as follows:

$$
r=\frac{\sum x y-\sum x \sum y / n}{\sqrt{\left[\sum x^{2}-\frac{\left(\sum x\right)^{2}}{n}\right]\left[\sum y^{2}-\frac{\left(\sum y\right)^{2}}{n}\right]}}
$$


where $x$ and $y$ represent the arterial blood pressure data points and the adjusted PTT data points, respectively. $n$ is the length of the data points and the value of $r$ supposed to be in the range from 0 to 1 . When $r$ is greater than 0.8 , it can be determined that the two sets of data points have strong correlation [79].

The correlation $r$ is only suitable for linear regression, while $R^{2}$ can be used to evaluate both linear and nonlinear regression. $R^{2}$ provides information about the goodness of the regression curve in fitting the model [79].

\subsection{The Procedure of Secondary Peak Detection}

The simulations of this chapter are taken on one hundred patients, sixty-five being male and thirty-five female, aging from 60 to 80 years. The data points of the physiological signals are extracted from the MIMIC II database. The synchronism of the arterial blood pressure signal, ECG signal, and finger PPG signal for each record are guaranteed. Each record consists of around ten segments. Each segment lasts ten seconds and has an average of ten cycles. By obtaining the signals in this way, the range of the SBP and DBP for each patient can cover from $80 \mathrm{mmHg}$ to $150 \mathrm{mmHg}$ and $60 \mathrm{mmHg}$ to $110 \mathrm{mmHg}$, respectively. The ECG signals are recorded at 125 sampling frequency and with 8 to 12-bit resolution [136].

\subsubsection{Secondary Peak Detection}

Three types of secondary peak detection methods are proposed in this chapter: SCF, GCF, and ACF methods. One patient (No. 3302803 in MIMIC II database) without visible secondary peak of the original PPG signal is chosen to show the detection procedure of the three methods. 
Figure 4.9 illustrates the peak detection procedure of the SCF method. The blue curve, red curve, and green curve represent the original PPG, the fitting curve, and the secondary peak detected from the original PPG signal, respectively. The SCF method is the first method we tested for visualizing the secondary peak. The peak shifting phenomenon is not considered, so the position of the primary peak of the fitting curve is the same as the main peak of the original signal.
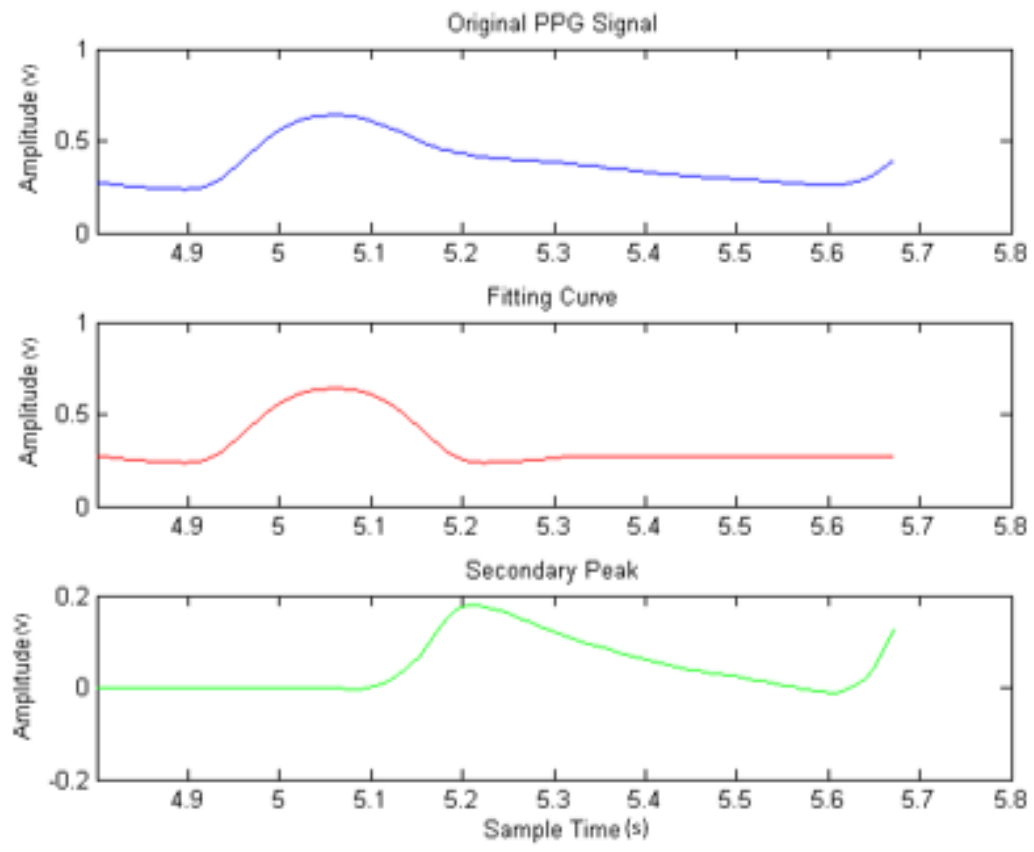

Figure 4.9: Secondary peak detection procedure of SCF method

In Figure 4.10, the GCF method is employed to detect the secondary peak of the original PPG signal. The second figure describes the second derivative of the original PPG signal with the zero-crossing points of the second derivative curve. The zero-crossing points are used to calculate the position and the standard deviation of the Gaussian fitting curve. Applying the value of $x_{1}$ and $x_{2}$ obtained from the second plot, the Gaussian fitting 
curve is determined as the red curve shown in the third plot. The secondary peak of the PPG signal is shown in the fourth plot. We can see that there is a concave segment in the secondary peak due to the amplitude difference between the original PPG signal and the Gaussian fitting curve.
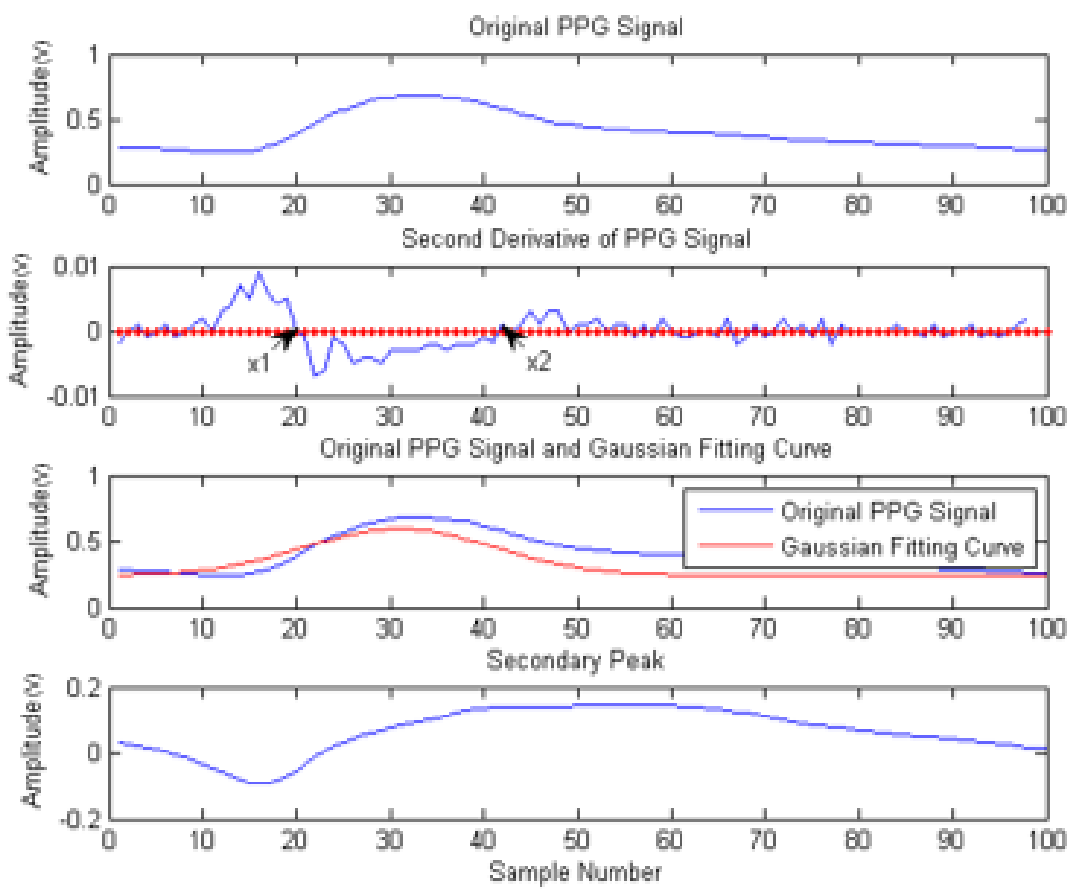

Figure 4.10: Secondary peak detection procedure of GCF method

Based on SCF and GCF, the ACF method is developed for the secondary peak detection. In Figure 4.11, the original PPG signal and the adaptive fitting curve are shown in the first plot. The value of the mean and the standard deviation of the fitting curve are adapted to fit the position and the upstroke side of the main peak. The peak shifting problem is clearly displayed as the location of the peak of the fitting curve is different with the location of the peak of the original PPG signal. 

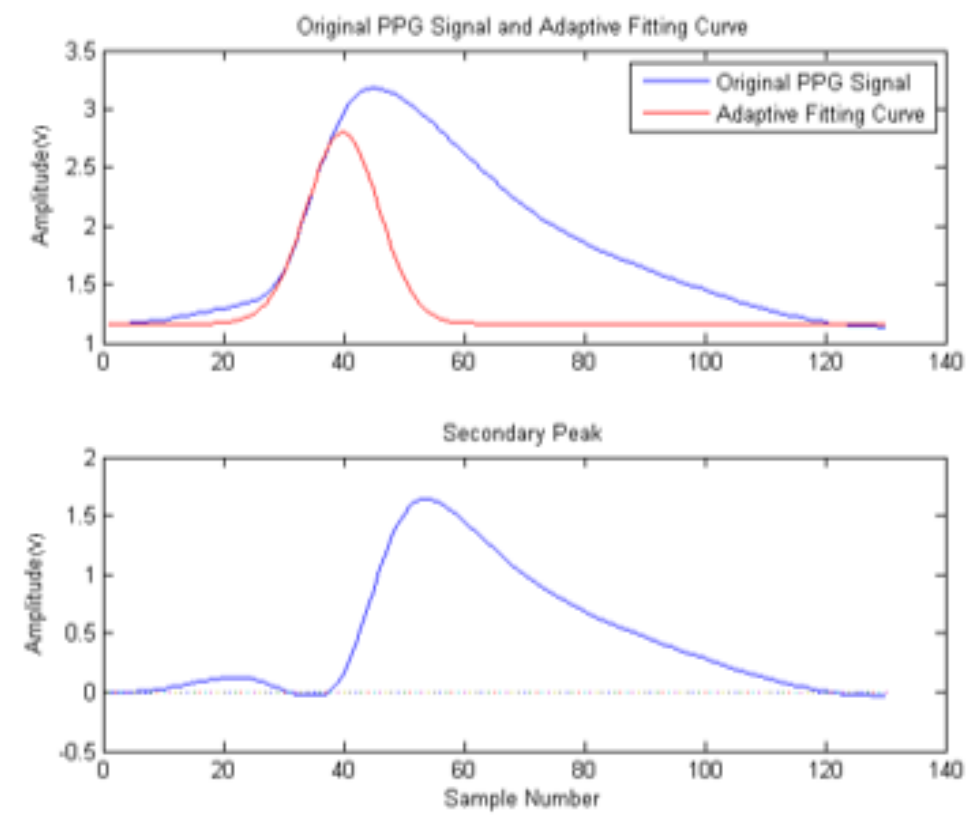

Figure 4.11: Secondary peak detection procedure of adaptive curve fitting method

\subsection{Detection Ability Demonstration of Proposed Methods}

\subsubsection{Different Scenarios}

To illustrate the peak shifting phenomena, three different scenarios are set up. One is two Gaussian curves with the same standard deviation and amplitude but different mean. One is two Gaussian curves with the same standard deviation but different mean and amplitude. One is two Gaussian curves with different standard deviation, mean, and amplitude. 


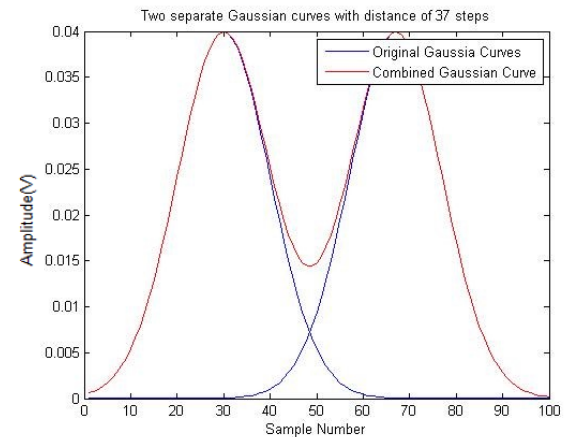

(a) Distance of 37 steps

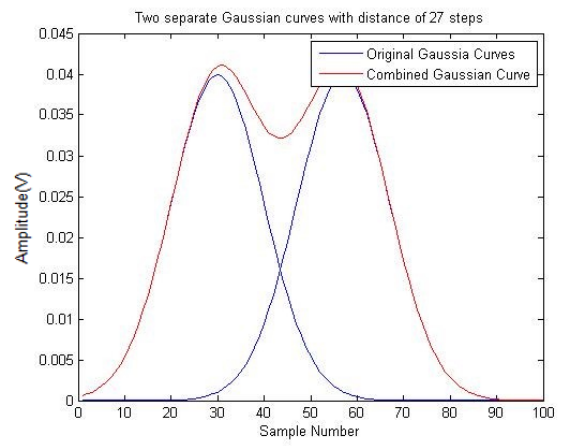

(c) Distance of 27 steps

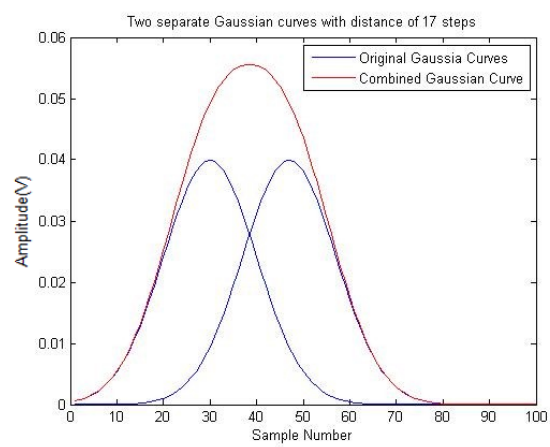

(e) Distance of 17 steps

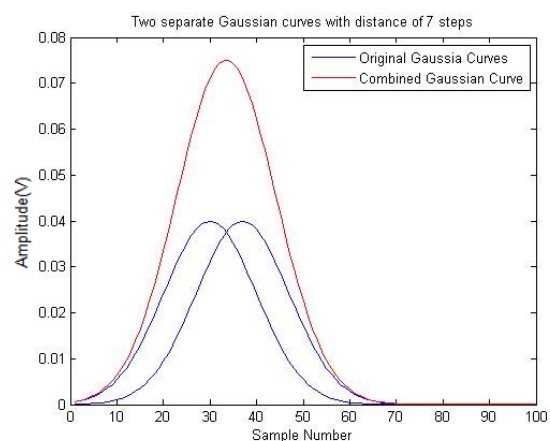

(g) Distance of 7 steps

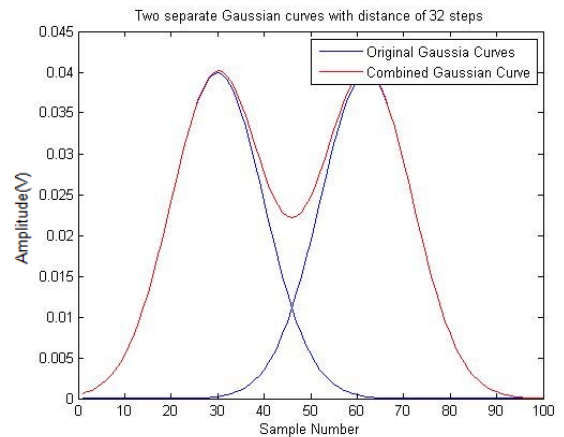

(b) Distance of 32 steps

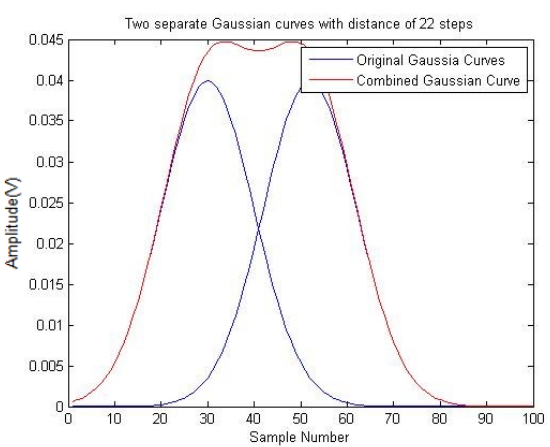

(d) Distance of 22 steps

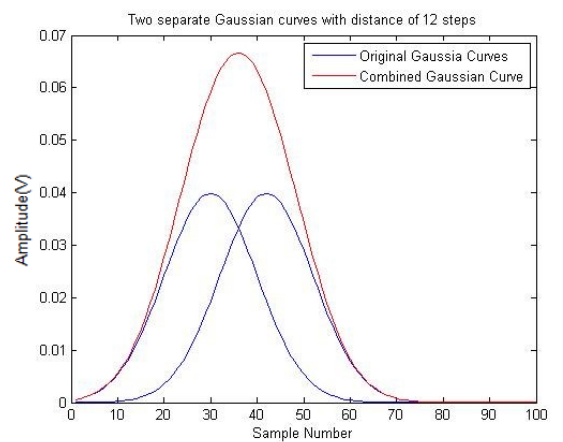

(f) Distance of 12 steps

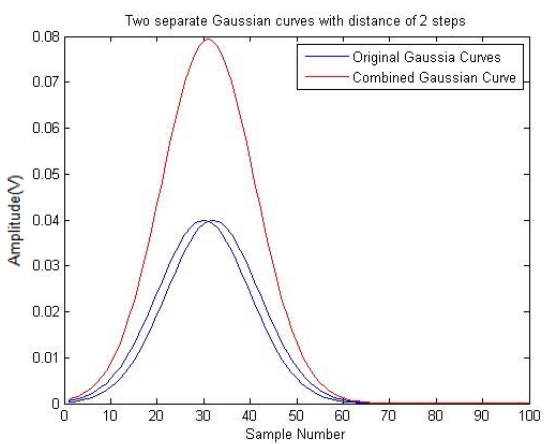

(h) Distance of 2 steps

Figure 4.12: Two Gaussian curves with same standard deviation and amplitude but different mean 
Figure 4.12 shows the demonstration of two Gaussian curves with the same standard deviation and amplitude but different mean merging together, causing the disappearance of the secondary peak. The range of the mean difference is from 2 to 40 sample numbers or steps.

From Fig. 4. 12 (a) to (h), it can be seen that when the two Gaussian curves get closer, the primary peak and the secondary peak start merging into one main peak with greater amplitude. This is getting more and more obvious when the mean difference is less than 17 steps with fixed standard deviation.

Figure 4.13 is the scenario when the two original Gaussian curves are with the same standard deviation but different mean and amplitude.

Figures in Figure 4.13 (a) to (h) display another scenario of peak shifting problem. With fixed standard deviation, when the distance of the two Gaussian curves in this scenario is less than 22 steps, the two peaks merge into one. And it can be seen that the location of the primary peak is changed.

The third scenario is when the two original Gaussian curves are with different standard deviation, different mean, and different amplitude. The examples are shown in Figure 4.14. 


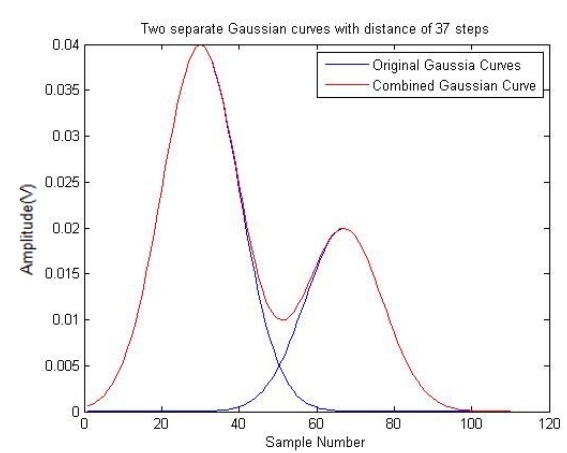

(a) Distance of 37 steps

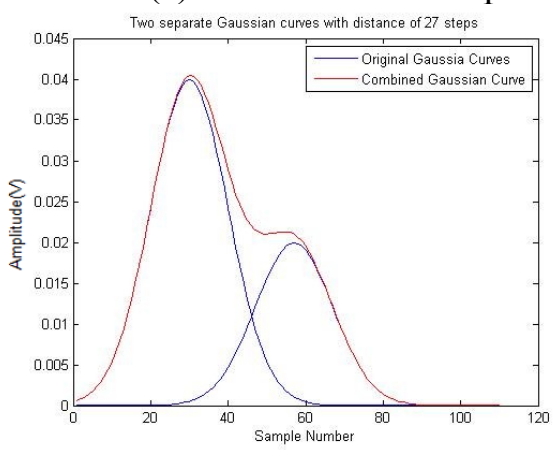

(c) Distance of 27 steps

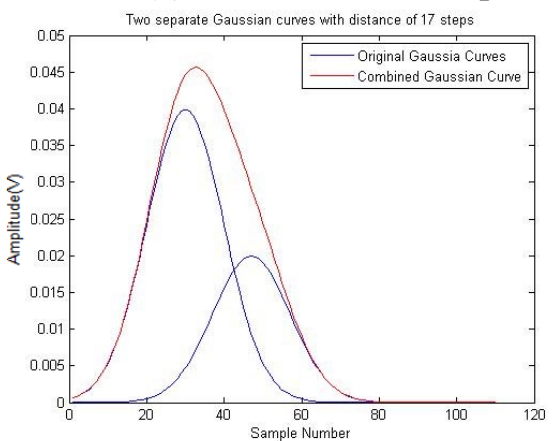

(e) Distance of 17 steps

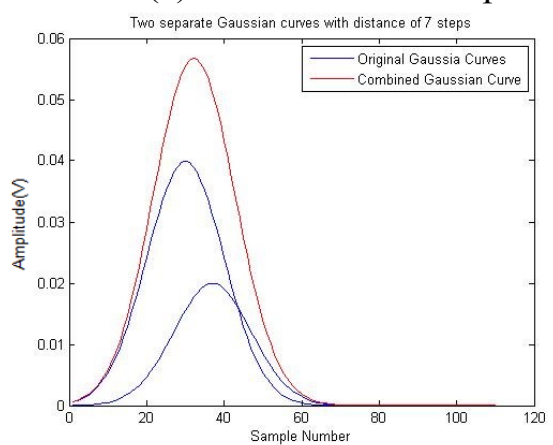

(g) Distance of 7 steps

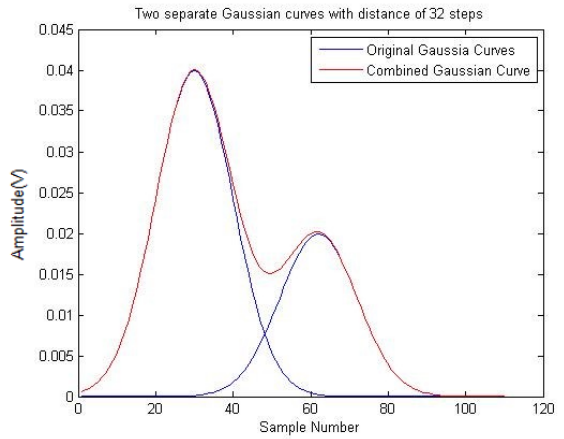

(b) Distance of 32 steps

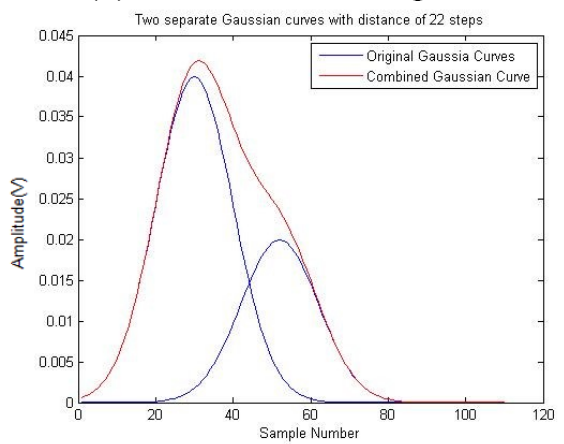

(d) Distance of 22 steps

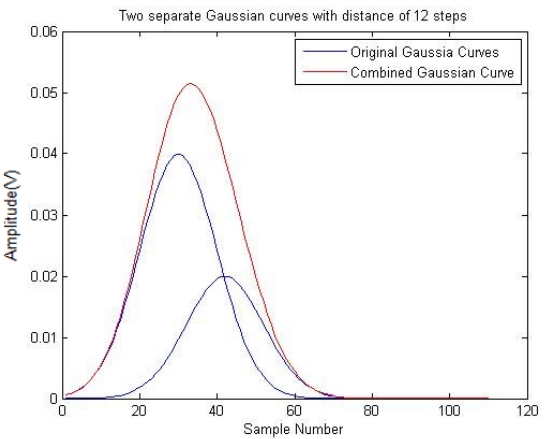

(f) Distance of 12 steps

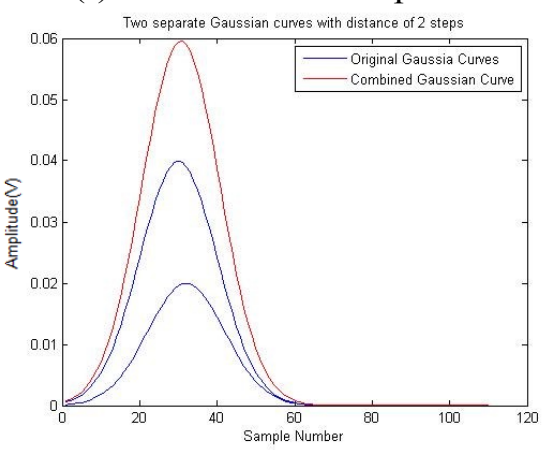

(h) Distance of 2 steps

Figure 4.13: Two Gaussian curves with same standard deviation but different mean and amplitude 


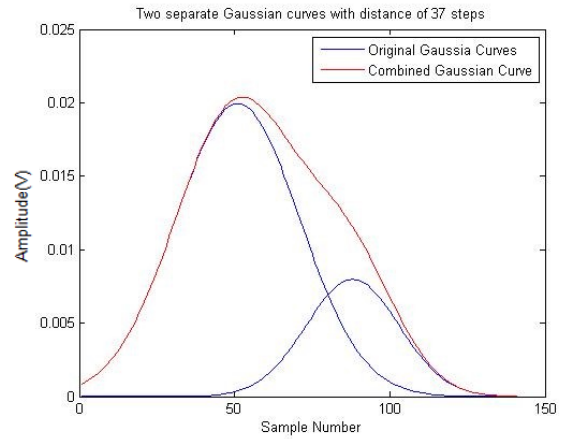

(a) Distance of 37 steps

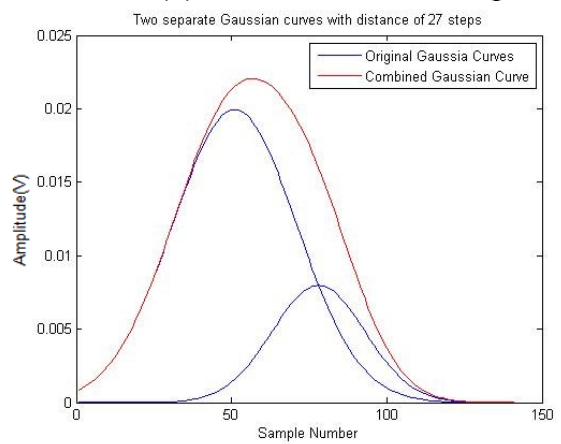

(c) Distance of 27 steps

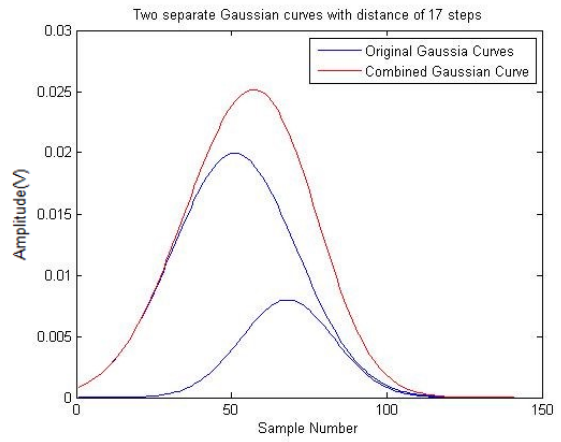

(e) Distance of 17 steps

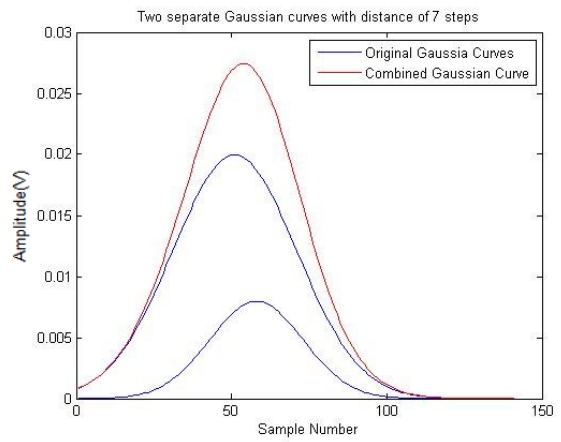

(g) Distance of 7 steps

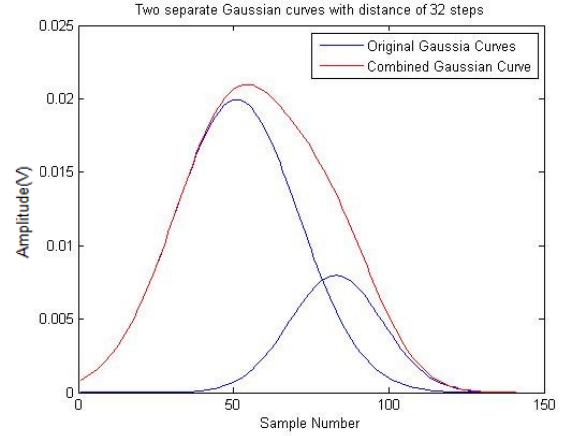

(b) Distance of 32 steps

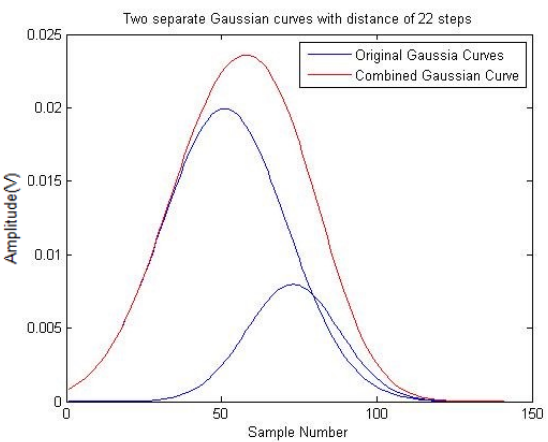

(d) Distance of 22 steps

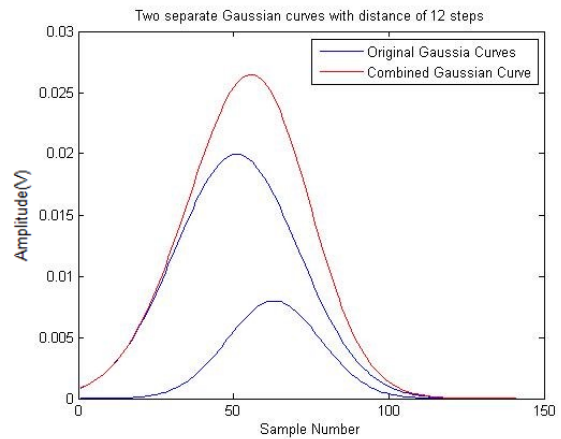

(f) Distance of 12 steps

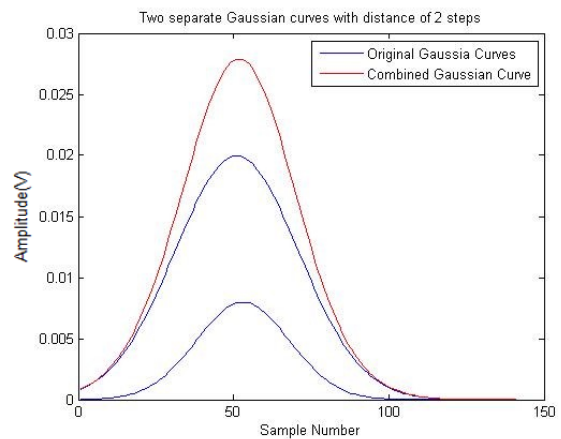

(h) Distance of 2 steps

Figure 4.14: Two Gaussian curves with different standard deviation, mean and amplitude 
From Fig. 4.14 (a) to (h), we can see that the peak shifting problem is more severe when the two Gaussian curves' standard deviation, amplitude, and mean are all different. Even when the distance between the two Gaussian curves are 37 steps, the two curves already merge together. If the difference between the standard deviation of the two Gaussian curves is greater, the merging phenomenon will be more significant. The original shape and amplitude of the two curves are difficult to be distinguished.

Figure 4.15 shows the peak detection thresholds for the standard deviation versus the distance between the two Gaussian curves under different amplitude ratios. $A 1$ and $A 2$ represent the amplitude of the two Gaussian curves, respectively. As the standard deviation getting larger, the two Gaussian curves are becoming much easier to get merged.

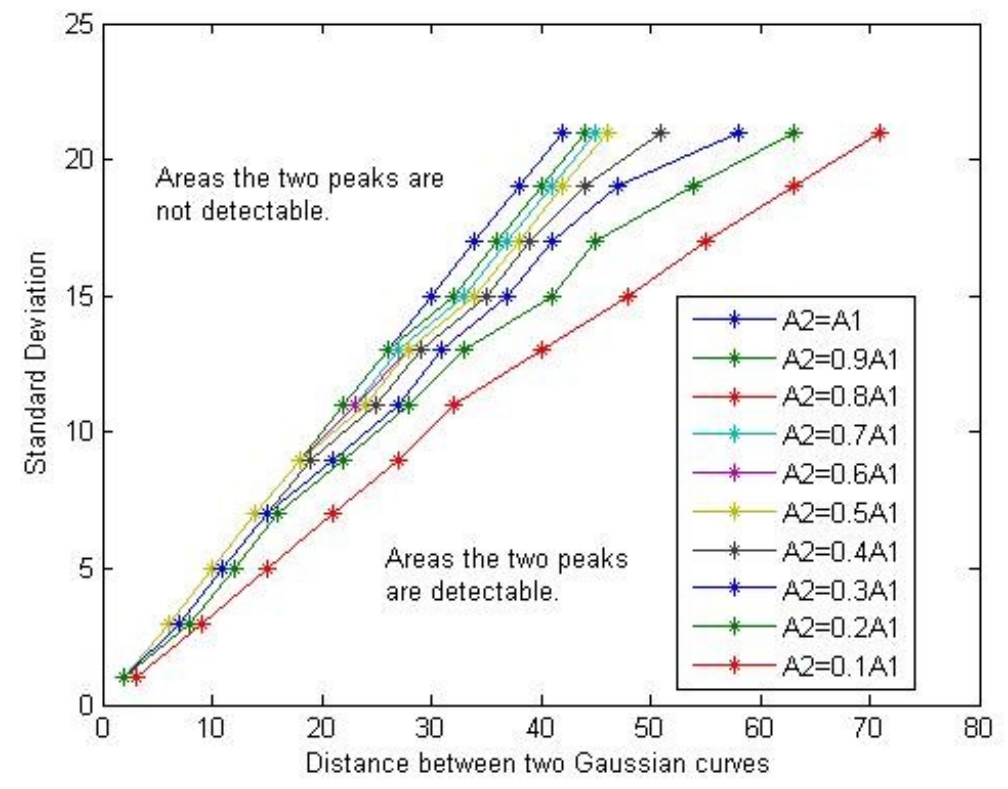

Figure 4.15: Peak detection thresholds for standard deviation verses the distance between the two Gaussian curves under different amplitude ratios 


\subsubsection{Curve Fitting Methods}

Three types of secondary peak detection methods are proposed in this study: symmetrical curve fitting method (SCF), Gaussian curve fitting method (GCF), and adaptive curve fitting method (ACF). The details of the procedure of these three methods are described in section 4.3. To demonstrate the performance of the three methods in detecting the primary peak, simulations are taken to apply these peak detection methods on the two merged Gaussian curves under different scenarios. The examples given below are when the distance between the two Gaussian curves is 17 steps.

The first scenario considered is the two Gaussian curves with the same standard deviation and amplitude but different means. Since the original Gaussian curves have the same standard deviation and amplitude, the synthetic curve generated by these two Gaussian curves merging together is symmetrical. When applying the SCF method, the symmetry of the merged curve determines that the fitting curve would have the same shape with the synthetic curve. This will cause the failure in detecting the secondary peak. The detection results by using SCF, GCF, and ACF, are shown in Figure 4.16 (a) to (c).

From Figure 4.16 we can see that if the original PPG signal has a perfect symmetric shape, SCF cannot be used to detect the primary peak. GCF and ACF can detect the primary peak and the secondary peak from the synthetic curve and ACF has better performance. The absolute differences between the original two Gaussian curves and the fitting curve and the secondary wave are given in Table 4.1 . 


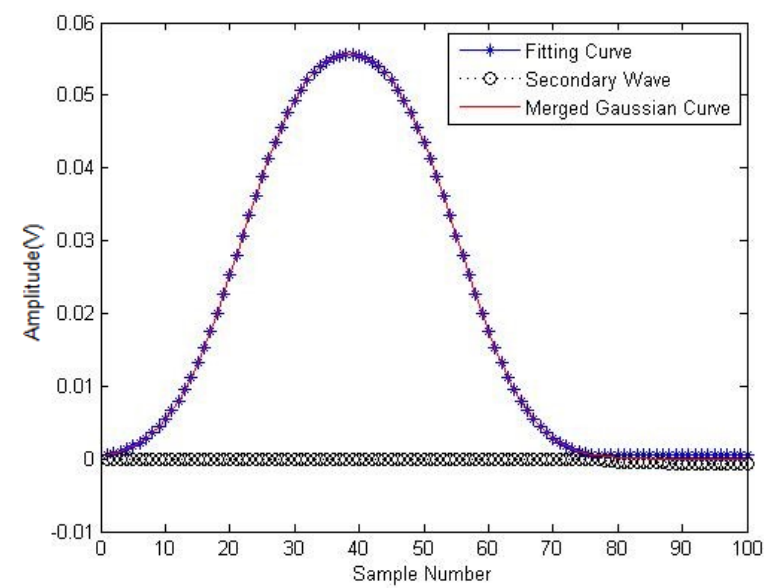

(a) $\mathrm{SCF}$

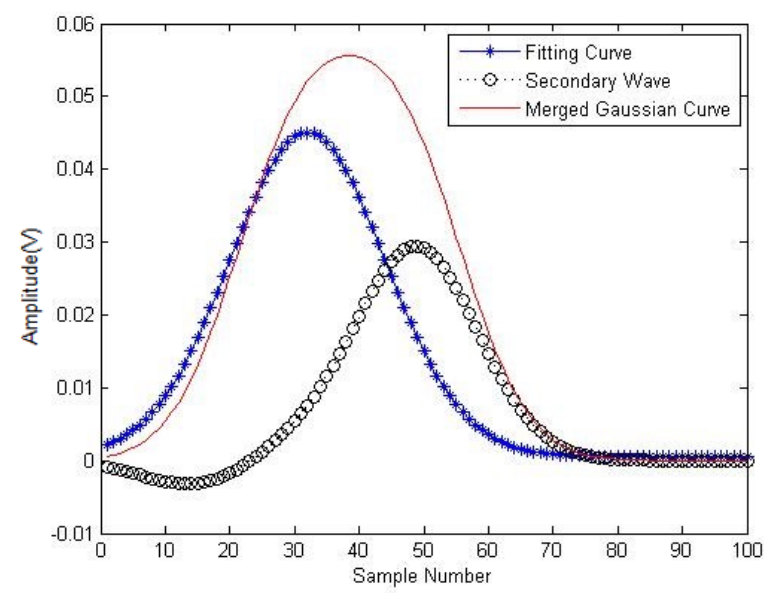

(b) GCF

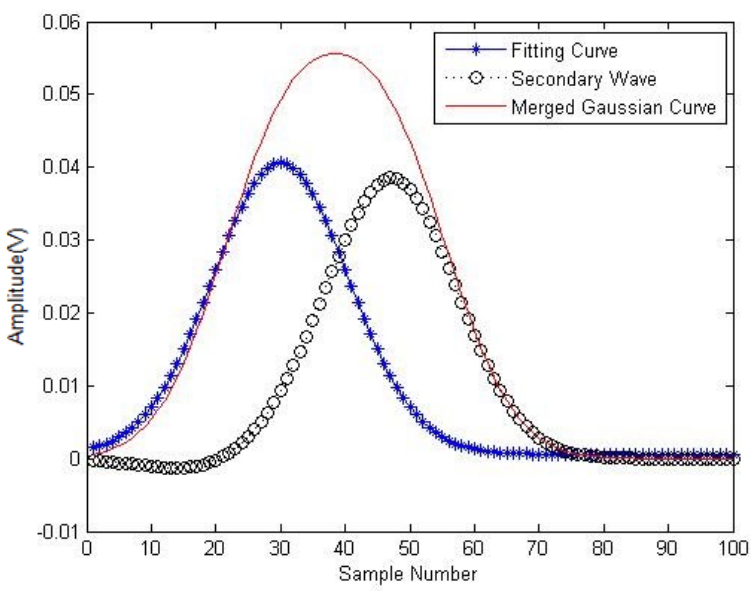

(c) $\mathrm{ACF}$

Figure 4.16: Secondary peak detection methods applied on curve composed of two Gaussian curves with the same standard deviation and amplitude but different mean 
Table 4.1: Absolute difference between original Gaussian curves and the fitting curves by using different methods

\begin{tabular}{cccc}
\hline & $\mathrm{SCF}\left(* 10^{-3}\right)$ & $\mathrm{GCF}\left(* 10^{-3}\right)$ & $\mathrm{ACF}\left(* 10^{-3}\right)$ \\
\hline Gaussian Curve one & 10.127 & 3.928 & 1.118 \\
Gaussian Curve two & 10.127 & 3.332 & 0.522 \\
\hline
\end{tabular}

Another scenario is two Gaussian curves with same standard deviation but different amplitude and mean mixing together. Figure 4.17 illustrates the performance of each dicrotic notch detection method.

All of the three detection methods can reveal the secondary wave in this scenario. By adding the adaptive procedure in the ACF method, Figure 4.17 (c) shows a better fitting curve and the secondary wave. The difference between the first Gaussian curve and the fitting curve and the difference between the second Gaussian curve and the revealed secondary wave are displayed in Table. 4.2.

Table 4.2: Absolute difference between original Gaussian curves and the fitting curves by using different methods

\begin{tabular}{lccc}
\hline & $\mathrm{SCF}\left(*^{*} 10^{-3}\right)$ & $\mathrm{GCF}\left(* 10^{-3}\right)$ & $\mathrm{ACF}\left(* 10^{-3}\right)$ \\
\hline Gaussian Curve one & 3.517 & 1.873 & 1.103 \\
Gaussian Curve two & 3.517 & 1.434 & 0.807 \\
\hline
\end{tabular}




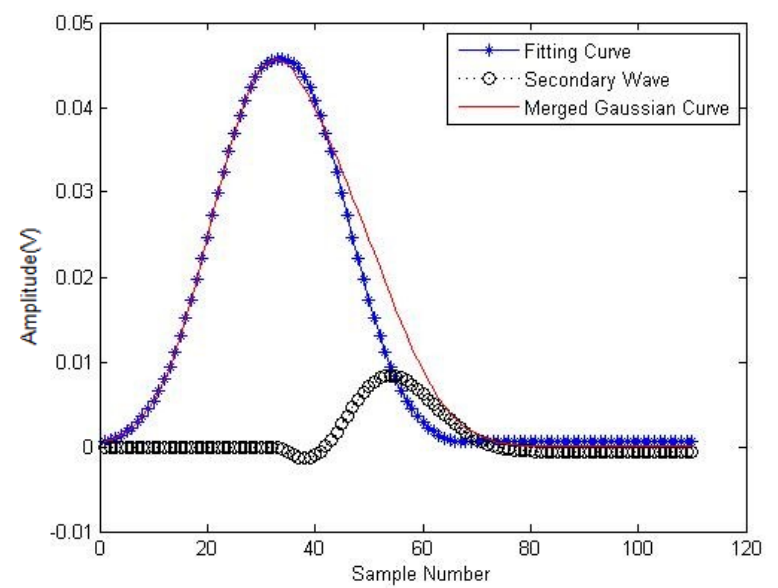

(a) $\mathrm{SCF}$

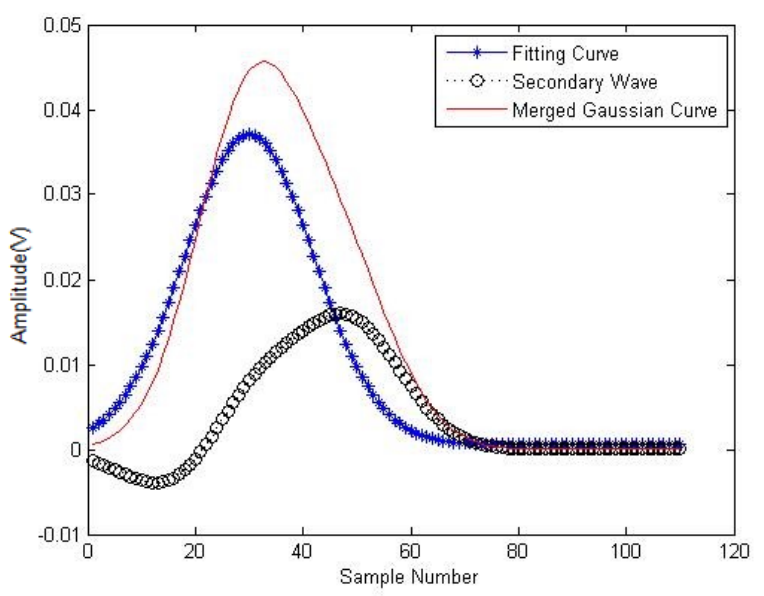

(b) GCF

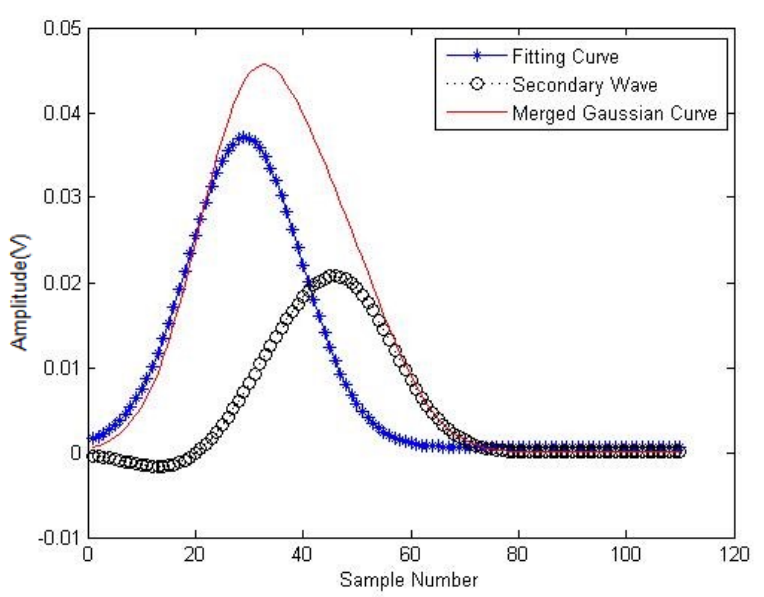

(c) $\mathrm{ACF}$

Figure 4.17: Secondary peak detection methods applied on curve composed of two Gaussian curves with the same standard deviation but different amplitude and mean 
All of the three detection methods can reveal the secondary wave in this scenario. By adding the adaptive procedure in the ACF method, Figure 4.17 (c) shows a better fitting curve and the secondary wave. The difference between the first Gaussian curve and the fitting curve and the difference between the second Gaussian curve and the revealed secondary wave are displayed in Table. 4.3.

Table 4.3: Absolute difference between original Gaussian curves and the fitting curves by using different methods

\begin{tabular}{lccc}
\hline & $\mathrm{SCF}\left(* 10^{-3}\right)$ & $\mathrm{GCF}\left(* 10^{-3}\right)$ & $\mathrm{ACF}\left(* 10^{-3}\right)$ \\
\hline Gaussian Curve one & 3.517 & 1.873 & 1.103 \\
Gaussian Curve two & 3.517 & 1.434 & 0.807 \\
\hline
\end{tabular}

A more complex scenario is that the original two Gaussian curves are with different standard deviation, different amplitude, and different mean. Generally speaking, the primary wave and the secondary wave of the PPG signals obtained from medical devices are totally different, because they are concerned with systole or diastole and blood flow reflections from the periphery. The detection performances of the three proposed methods are shown in Figure 4.18 (a) to (c). 


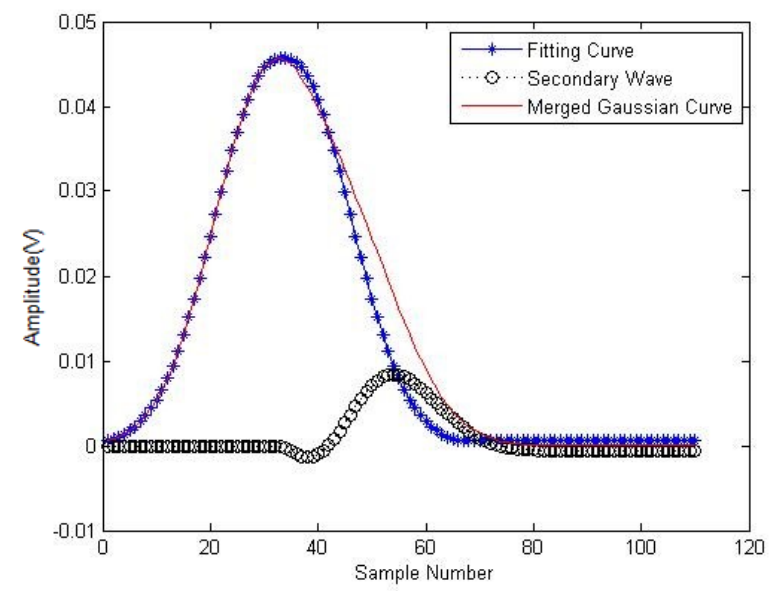

(a) $\mathrm{SCF}$

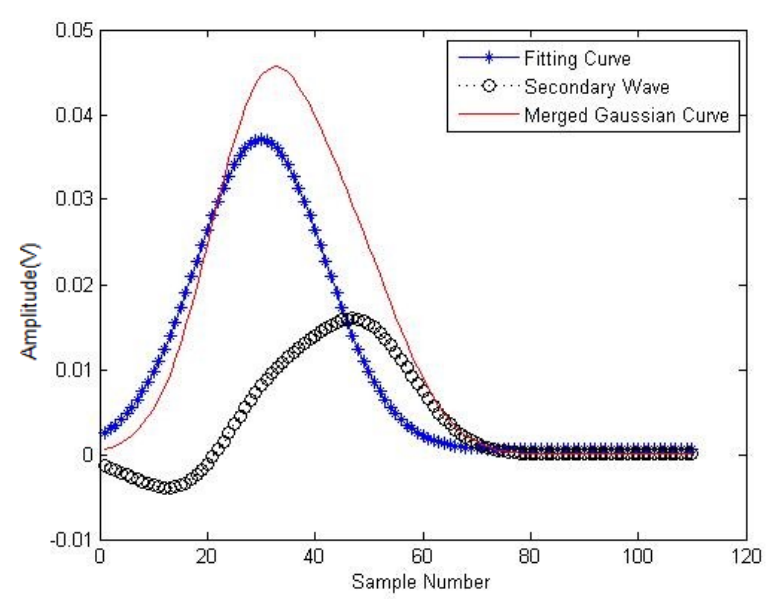

(b) GCF

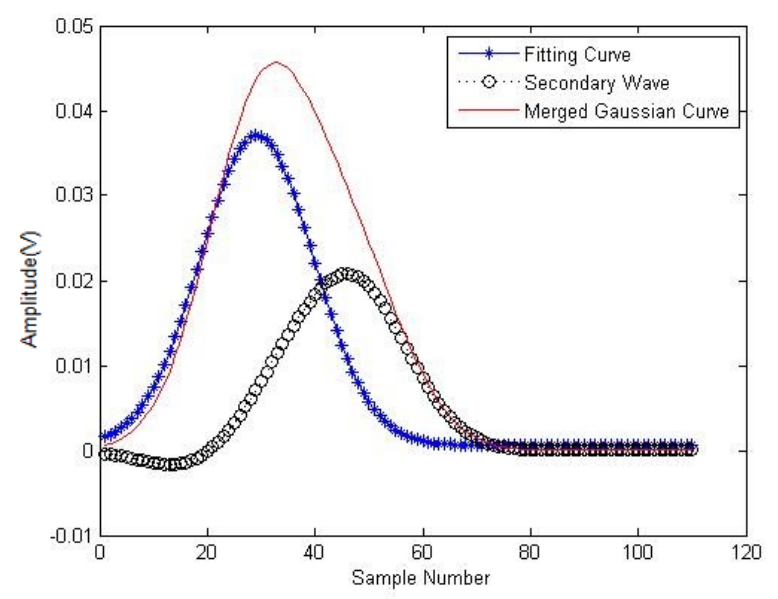

(c) $\mathrm{ACF}$

Figure 4.18: Secondary peak detection methods applied on Gaussian curve composed of two Gaussian curves with different standard deviation, amplitude, and mean 
Figure 4.18 demonstrates that SCF, GCF, and ACF can detect the dicrotic notch and distinguish the two Gaussian curves. ACF has better detection ability than the other two methods in general. Also the absolute difference between the first Gaussian curve and the fitting curve and the absolute difference between the second Gaussian curve and the secondary wave are calculated. The results are shown in Table 4.4.

Table 4.4: Absolute difference between original Gaussian curves and the fitting curves by using different methods

\begin{tabular}{lccc}
\hline & $\mathrm{SCF}\left(* 10^{-3}\right)$ & $\mathrm{GCF}\left(* 10^{-3}\right)$ & $\mathrm{ACF}\left(* 10^{-3}\right)$ \\
\hline Gaussian Curve one & 2.964 & 1.975 & 0.864 \\
Gaussian Curve two & 2.964 & 1.164 & 0.590 \\
\hline
\end{tabular}

From the above simulations, the detection ability of our proposed secondary peak detection methods has been demonstrated. SCF, GCF, and ACF can separate the original Gaussian curves from the synthetic curve, except one condition that SCF cannot detect the dicrotic notch when the two Gaussian curves are with the same standard deviation and amplitude. From the calculated absolute differences, it can be seen that the more different the two original Gaussian curves are, the better the performance of the proposed methods have. In medical examination or self-monitoring situation, the PPG signals can be usually regarded as containing two different waves, the primary wave and the secondary wave. The proposed SCF, GCF, and ACF methods can be used to reveal the dicrotic notch from the embedded secondary wave. And the ACF method has better detection effect. 


\subsection{Primary Peak Location Analysis}

Since the PPG signal can be modeled by four Gaussian curves, the potentiality of using the fitted Gaussian curves to localize the primary peak of the PPG signal is discussed in this part. Record of patient \#3011613 from the MIMICII database is used for simulations. Two different cycles are selected as shown in Figure 4.19 and Figure 4.20, respectively.

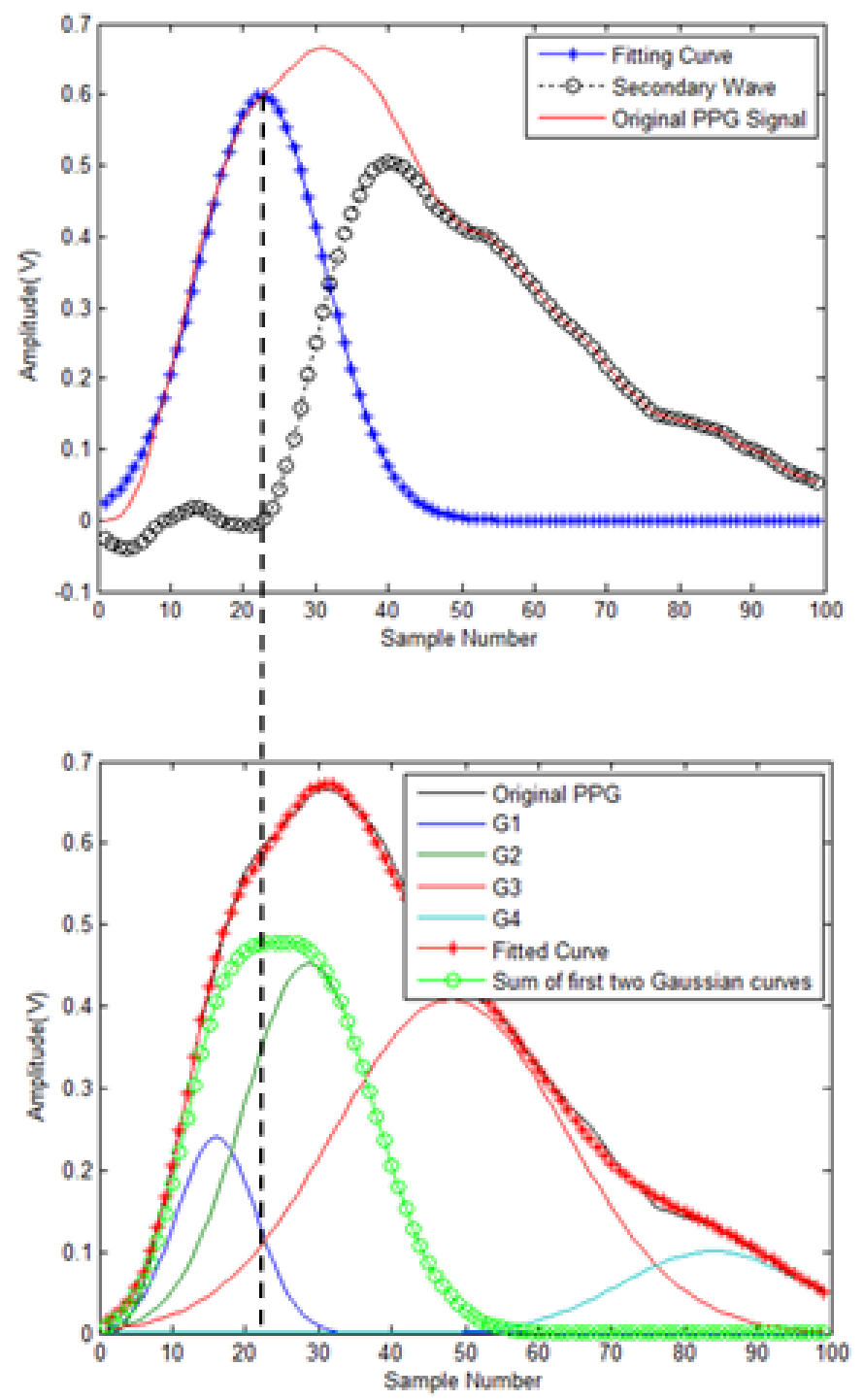

Figure 4.19: Primary peak location comparison between ACF and the four-Gaussiancurve modeling (cycle 1) 


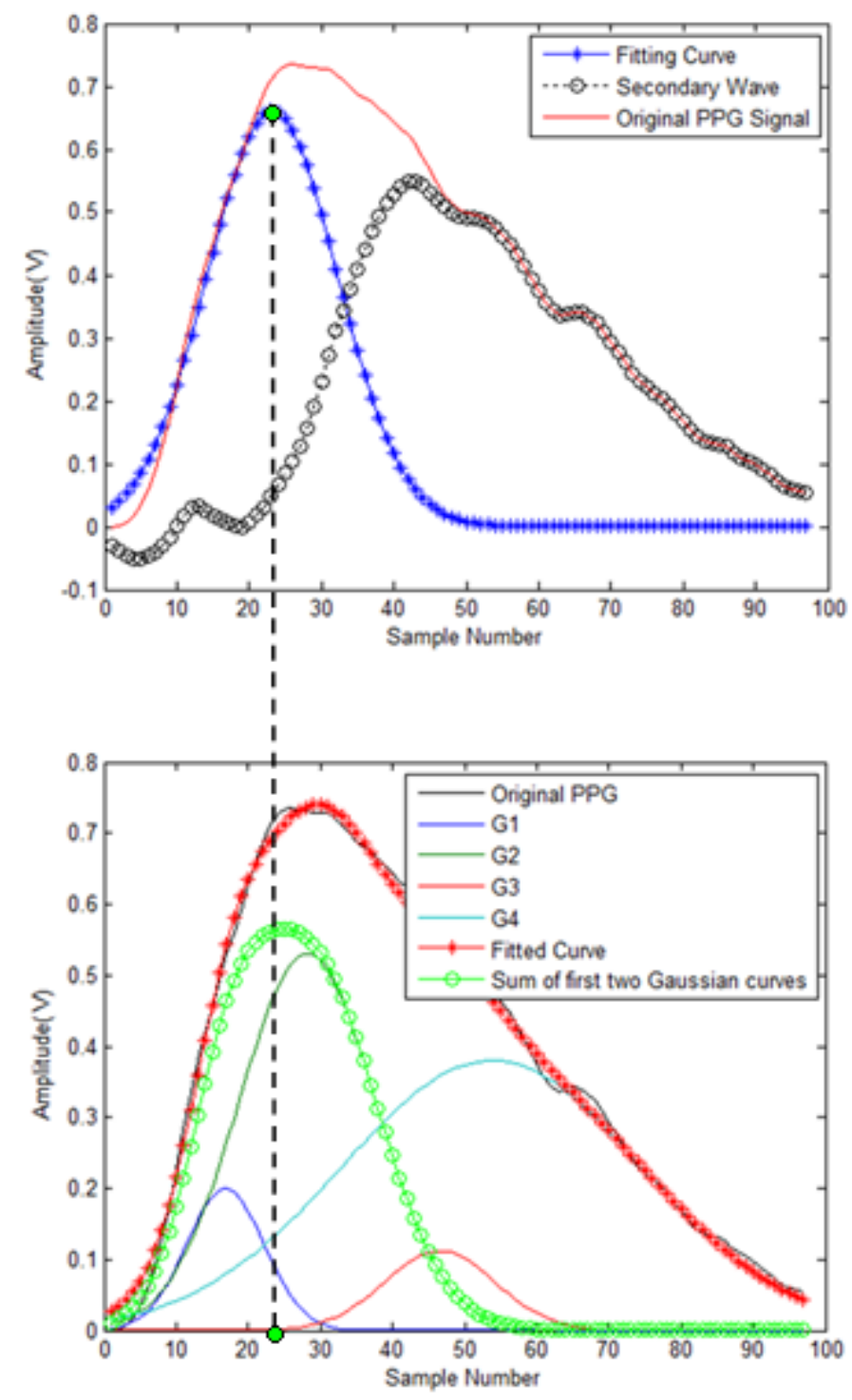

Figure 4.20: Primary peak location comparison between ACF and the four-Gaussiancurve modeling (cycle 2)

In Figure 4.19, the upper plot is using the adaptive curve fitting method to detect the secondary peak. The red, blue, and black curves represent the original PPG signal, the adaptive fitting curve, and the detected secondary wave, respectively. The location of the primary peak of the fitting curve is 22.5 . The lower plot is the four Gaussian curves 
calculated to model the original PPG signal. The green circle line stands for the summation of the first two Gaussian curves. The peak location of the green circle line is 23 .

As the same as in Figure 4.19, Figure 4.20 shows another cycle of the PPG signal. The primary peak of the ACF fitting curve is 23 . The location of the peak of the first two Gaussian curves' summation is 23.5 .

The preliminary results show high correlation between the locations of the peak detected by the ACF method and the peak of the first two Gaussian curves' summation by the four-Gaussian-curve modeling. So there is a high potentiality that the four-Gaussiancurve modeling can be applied as another efficient way to analyze the arterial blood pressure.

\subsection{Summary and Conclusion}

The arterial blood pressure is one of the most important physiological parameters for pathologic diagnosis and healthcare monitoring. PTT is a commonly used parameter for arterial blood pressure measurement. Usually, PTT is defined as the delay between the Rpeak of the ECG signal and the following primary peak of the corresponding PPG signal. Sometimes the dicrotic notch and the secondary peak of the PPG signal is not visible, leading to the peak shifting of the primary peak of the PPG signal. This will impact the accuracy of the PTT value and is very severe when PTT is used for blood pressure estimation.

In this chapter, Gaussian curves were employed to model the PPG signal, given the nature of the contour of the PPG signal. The absolute differences between the original 
Gaussian curves and the modeled curves were calculated. Three types of secondary peak detection methods were developed, which were symmetrical curve fitting method, Gaussian curve fitting method, and adaptive curve fitting method. To illustrate the peak detection performance of the proposed algorithms, Gaussian curves were applied to simulate the peak shifting phenomena and different scenarios were considered. The fourGaussian-curve modeling showed high potential in estimating the arterial blood pressure. 


\section{Chapter 5}

\section{Correlation between Arterial Blood Pressure and Pulse}

\section{Transit Time}

The arterial blood pressure is an essential physiological parameter for health monitoring. The conventional method to measure the systolic and the diastolic blood pressure is through the inflation and the deflation of the bladder and the brachial cuff [11]. This method may cause anxiety to the users which in return can affect the blood pressure (white coat syndrome). Also, the cuff-based method is not suitable for continuous monitoring of the blood pressure.

Pulse transit time (PTT) has been reported to have a high correlation with arterial blood pressure and has been proven to be useful as a continuous, noninvasive, and indirect technique to estimate the arterial blood pressure [23].

In this chapter the real electrocardiogram (ECG) signal, photoplethysmograph (PPG) signal, and the arterial blood pressure signal are extracted from the MIMICII database. PTT is defined as the delay between the R-peak of the ECG signal and the primary peak of the 
following finger PPG signal. After acquiring the enhanced ECG and PPG signal as described in chapter 3 and chapter 4, respectively, the adjusted PTT is calculated in this chapter. The correlation between the adjusted PTT and the arterial blood pressure is compared with the correlation between the PTT, which is obtained from the original ECG and PPG signals from the MIMIC database, and the arterial blood pressure. The simulation results show a high correlation between the arterial blood pressure and the adjusted PTT. Different polynomial regression curves are applied for further demonstration. The reliability of the regression curves are tested.

\subsection{Signal Analysis}

In this chapter, ten individual records were first extracted from the MIMICII database to study the relationship between the arterial blood pressure and the PTT. These records include the synchronized arterial blood pressure signal, the ECG signal, and the finger PPG signal. Each record contains ten segments. Each segment lasts ten seconds and has an average of ten cycles, which can be seen from Figure 5.1.

For each patient, the ten segments can cover the range of SBP value between 80 $\mathrm{mmHg}$ and $150 \mathrm{mmHg}$, and the DBP value between $60 \mathrm{mmHg}$ and $110 \mathrm{mmHg}$. The ECG signal was recorded at 500 samples per second with 12-bit resolution.

In order to demonstrate that the PTT and the SBP, DBP, and mean blood pressure (MBP) have high correlation and reduce the interference from other issues, the signals used for simulations are selected from the relatively clean portions of each patient's record. The tool used to extract the signals from the database is the PhysioBank ATM, which has 
several toolboxes, including plotting the waveforms of the specific signal, showing samples as text, and exporting signals in the format of .mat [138].
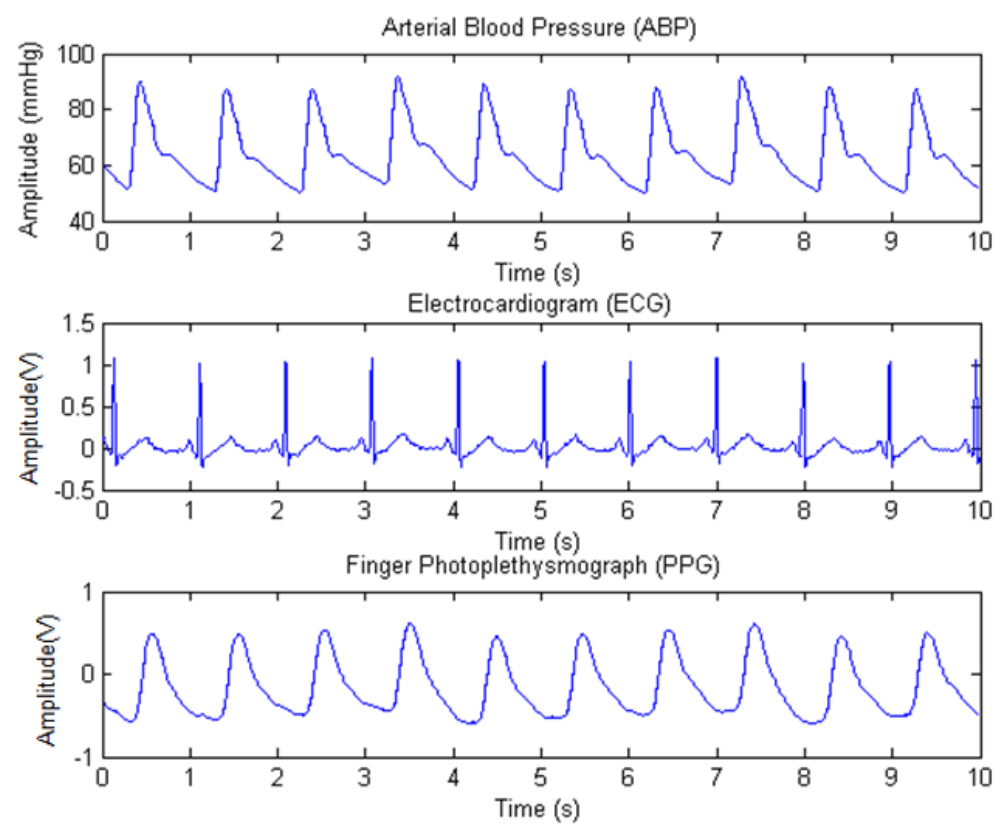

Figure 5.1: ABP, ECG, and PPG signal segment extracted from the MIMICII database

As mentioned above, the PTT is defined as the delay between the R-peak of the ECG signal and the primary peak of the following PPG signal. Some features of the ECG signal are sometimes hard to detect; especially, when there is noise contamination in the original ECG signal. Compared with the inconspicuous features of the ECG signal, R peaks are easier to observe due to its amplitude and the unique shape. $\mathrm{R}$ peaks are also more stable than the other wave peaks.

As shown in Figure 5.2, the red curve and the green curve represent the lead II ECG signal and the PPG signal, respectively. The time interval between the first red peak and the following green peak is the PTT. 


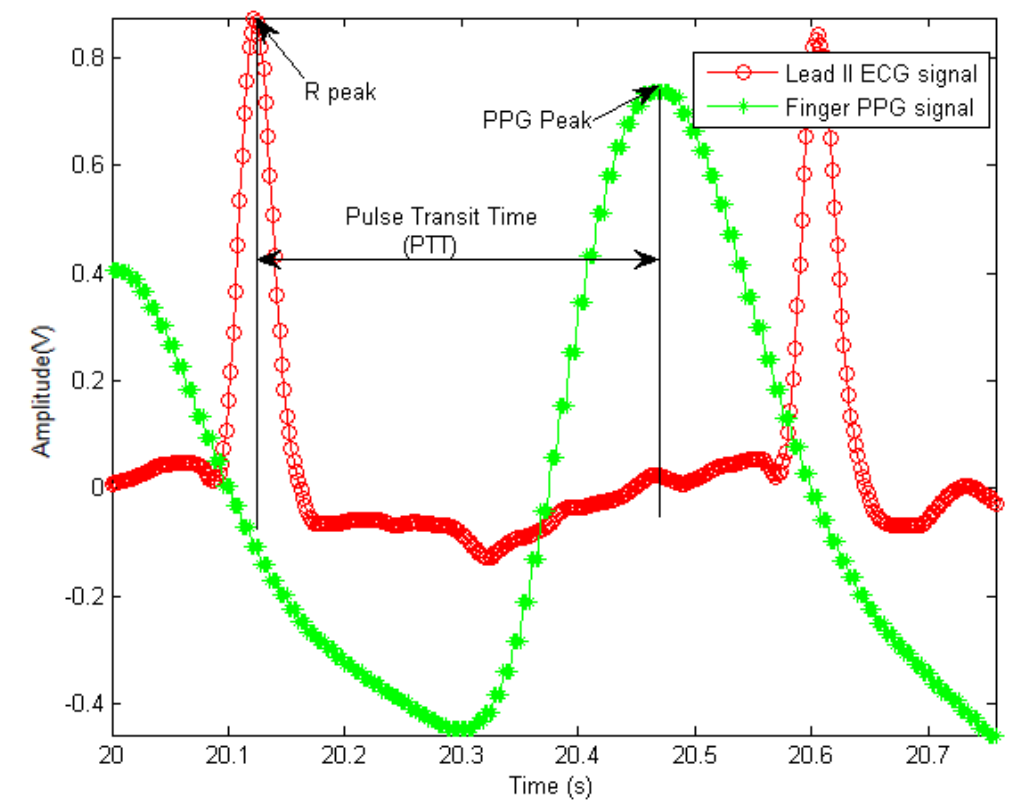

Figure 5.2: Selected ECG signal and finger PPG signal from MIMIC database. The definition of PTT is determined by the delay between the R-peak and the PPG peak

The processing procedure of this section involves several steps and can be described as follows:

Step 1) Select the relatively clean signal segments that have the specific blood pressure range.

Step 2) Detect the R-peak of the ECG signal and the primary peak of the PPG signal.

Step 3) Detect the maximum and minimum extremes of the blood pressure signal to obtain the SBP and the DBP.

Step 4) Determine the delay between each corresponding R-peak of the ECG signal and the primary peak of the PPG signal, which is the PTT. 
Step 5) Calculate the correlation values of SBP versus PTT, DBP versus PTT, and MBP versus PTT for each record. Draw the linear, quadric, and cubic regression curves.

Step 6) Compare the fitted regression curves and select the best one for future blood pressure estimation.
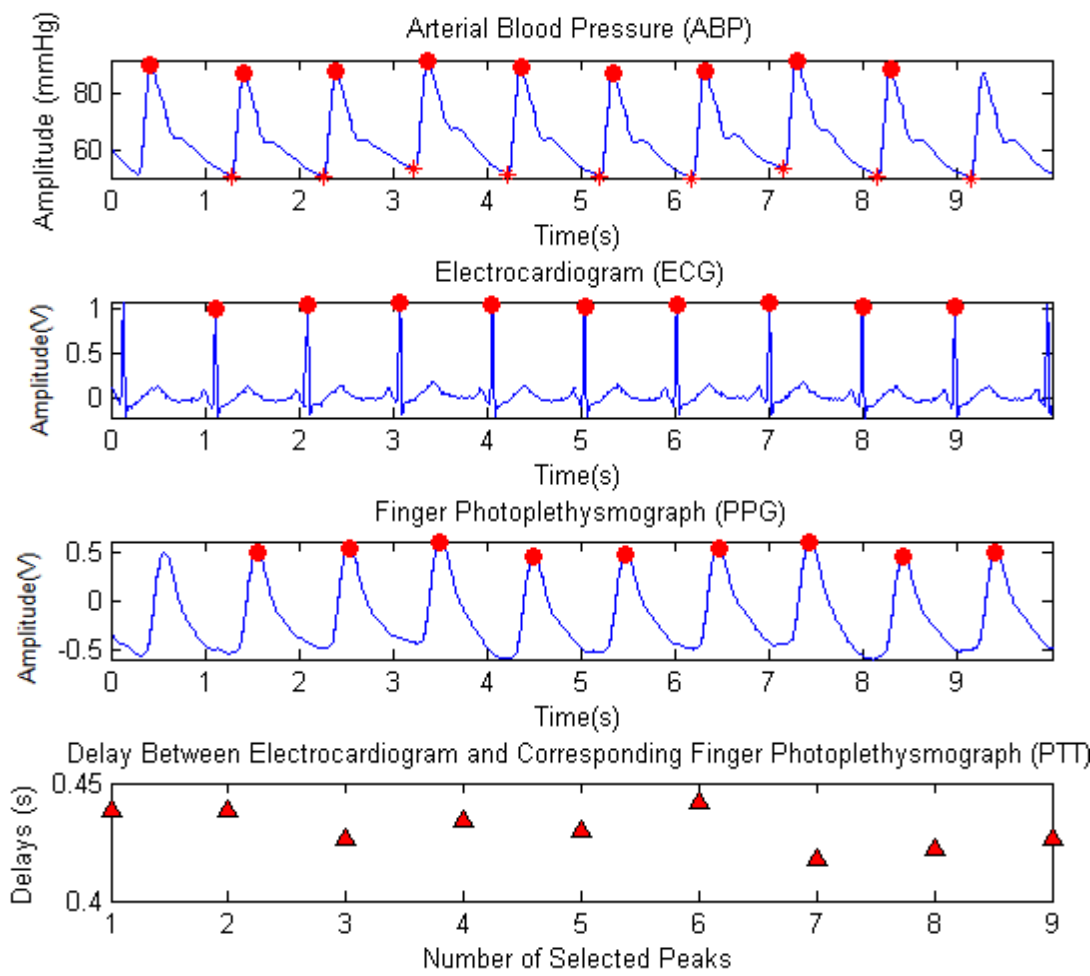

Figure 5.3: Extremes of ABP, R-peak of ECG, primary peaks of PPG, and the calculated PTT

Figure 5.3 shows the extremes of the blood pressure, the R-peak of the ECG signal, the primary peaks of the PPG signal, and the calculated PTT of one segment. It can be seen from the figure that when the SBP value is sticking around $90 \mathrm{mmHg}$, the PTT is stable with a value range of $0.42-0.43 \mathrm{~s}$. 


\subsection{BP-PTT Relation}

In order to evaluate the strength and direction of the relationship between the arterial blood pressure and the PTT, the scatter plot and the correlation $r$ are applied. Usually, there are two types of correlation: positive and negative [173]. Since the PTT decreases with the increase of the blood pressure, the direction of the correlation between these two signals is negative. When the value of $r$ is greater than 0.8 , it can be decided that the two sets of data points have strong correlation [173].

Taking record \#3302803 as an example, Figure 5.4 shows the scatter plot for the SBP versus PTT and the linear, quadric, and cubic regression curves. Each red dot is the PTT versus each corresponding arterial blood pressure value. The dashed graphs are the regression curves obtained from the data points in the same figure. It is obvious that all three regression curves can be used to estimate the SBP value, and the cubic regression curve fits the data points much better than the other two regression curves.
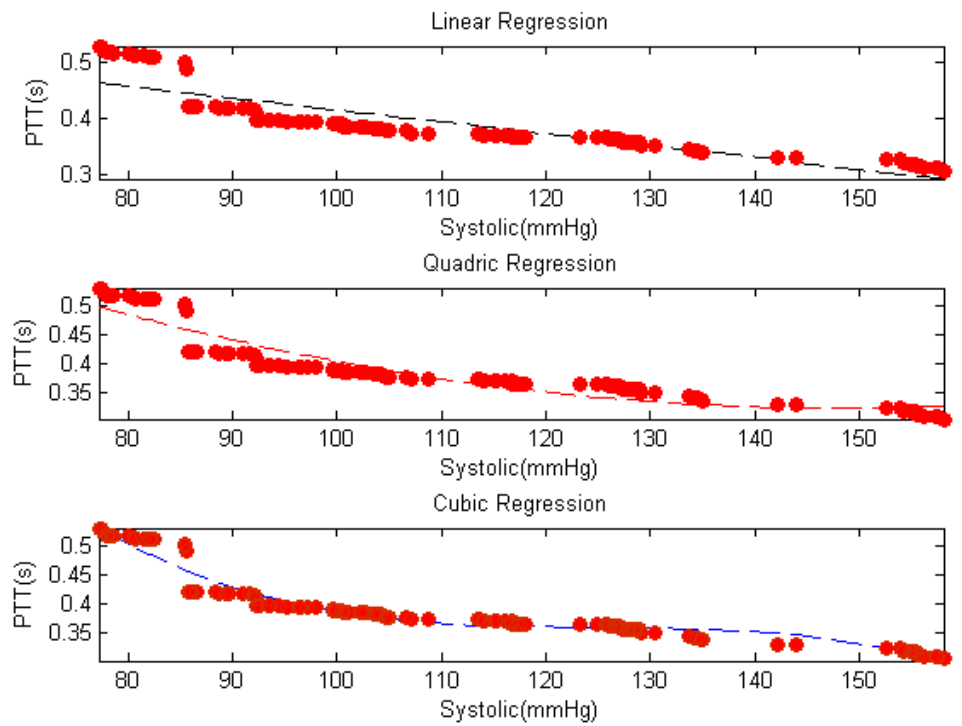

Figure 5.4: Scatter plot for the SBP versus PTT and three different types of regression curves 

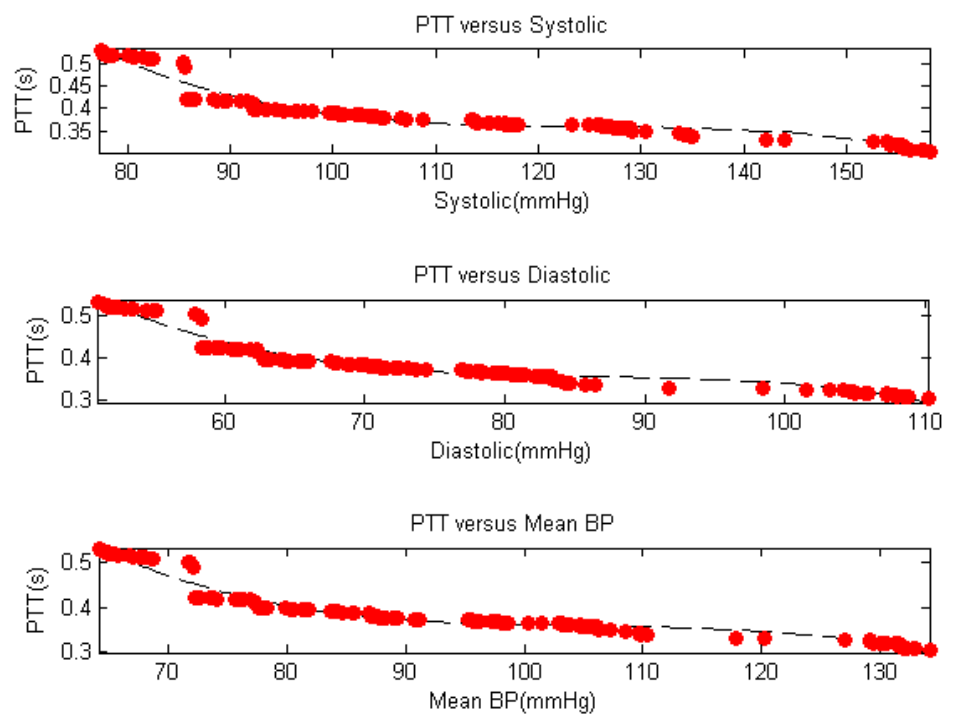

Figure 5.5: Scatter plot for PTT versus SBP, DBP, and MBP with cubic regression curve

Figure 5.5 illustrates the scatter plot for the SBP versus PTT, the DBP versus PTT, and the MBP versus PTT, respectively, by using cubic regression model. It can be demonstrated that the PTT does have strong correlation with different types of arterial blood pressure, including SBP, DBP, and MBP.

Table 5.1 exhibits the equations obtained from the PTT versus SBP data points by using linear regression, quadric regression, and cubic regression models, respectively. For patient 2, the equations are corresponding to the dashed curves in Figure 5.4. $x$ is the time delay between each ECG-PPG pair and $y$ represents the corresponding SBP value.

Table 5.1 presents the detailed analysis related to three patients. Preliminary results of the other seven patients show the same pattern. 
Table 5.1: Different regression models of SBP-PTT on three-patient samples

\begin{tabular}{|c|c|c|}
\hline & & Systolic Blood Pressure \\
\hline \multirow{3}{*}{ Patient 1} & $\begin{array}{c}\text { Linear } \\
\text { Regression }\end{array}$ & $y=-359.07 x+251.76$ \\
\hline & $\begin{array}{l}\text { Quadric } \\
\text { Regression }\end{array}$ & $y=2807.9 x^{2}-2725.4 x+738.3$ \\
\hline & $\begin{array}{c}\text { Cubic } \\
\text { Regression }\end{array}$ & $y=5850.1 x^{3}-4519.4 x^{2}+228.6 x+347.77$ \\
\hline \multirow{3}{*}{ Patient 2} & $\begin{array}{c}\text { Linear } \\
\text { Regression }\end{array}$ & $y=-991.48 x+523.65$ \\
\hline & $\begin{array}{c}\text { Quadric } \\
\text { Regression }\end{array}$ & $y=4928.8 x^{2}-5122.6 x+1387.3$ \\
\hline & $\begin{array}{c}\text { Cubic } \\
\text { Regression }\end{array}$ & $y=368090 x^{3}-459410 x^{2}+189740 x-25819$ \\
\hline \multirow{3}{*}{ Patient 3} & $\begin{array}{c}\text { Linear } \\
\text { Regression }\end{array}$ & $y=-652 x+339.28$ \\
\hline & $\begin{array}{c}\text { Quadric } \\
\text { Regression }\end{array}$ & $y=3954.4 x^{2}-3538.9 x+862.33$ \\
\hline & $\begin{array}{c}\text { Cubic } \\
\text { Regression }\end{array}$ & $y=20373 x^{3}-18908 x^{2}+4949.9 x-180.67$ \\
\hline
\end{tabular}

Table 5.2: The correlation between PTT and each type of ABP for three-patient samples

\begin{tabular}{|c|c|c|c|c|}
\hline \multicolumn{2}{|c|}{} & \multicolumn{3}{c|}{ Arterial Blood Pressure } \\
\cline { 3 - 5 } \multicolumn{2}{|c|}{} & Systolic & Diastolic & Mean \\
\hline \multirow{3}{*}{ Correlation } & Patient 1 & $87.5 \%$ & $87.7 \%$ & $87.8 \%$ \\
\cline { 2 - 5 } & Patient 2 & $95.1 \%$ & $94.0 \%$ & $94.5 \%$ \\
\cline { 2 - 5 } & Patient 3 & $93.7 \%$ & $94.2 \%$ & $94.7 \%$ \\
\hline
\end{tabular}

Table 5.2 displays the correlation values between the PTT and the SBP, DBP, and MBP of the three-patient samples, respectively. The average correlation for PTT versus SBP, DBP, and MBP of all the experimental subjects are greater than $87 \%$, which means that the PTT and the types of blood pressure we considered in this section has high 
correlation [173], and the PTT can be used as a measurement to estimate the noninvasive and cuff-less blood pressure.

\subsection{BP and Adjusted PTT}

In section 5.2, the ECG and PPG signals were regarded as noise-free signals and no denoising processing was done before the extraction of the characteristic parameters to calculate the PTT. The PTT obtained in this way was regarded as the original PTT. In this section, we take the de-noised version of the ECG signal and the dicrotic notch of the PPG signal into consideration. For the de-noising procedure, more details have been displayed in chapter 3 on enhancing the ECG signal by using the EMD-based algorithm and the adaptive filtering technology.

Before showing the scatter plot and the regression models, the correlation between the adjusted PTT and the blood pressure after applying our proposed PPG secondary peak detection methods in chapter 4 is illustrated by the intuitive plots. The information about the peak shifting phenomenon and the adjusted PTT is also provided.
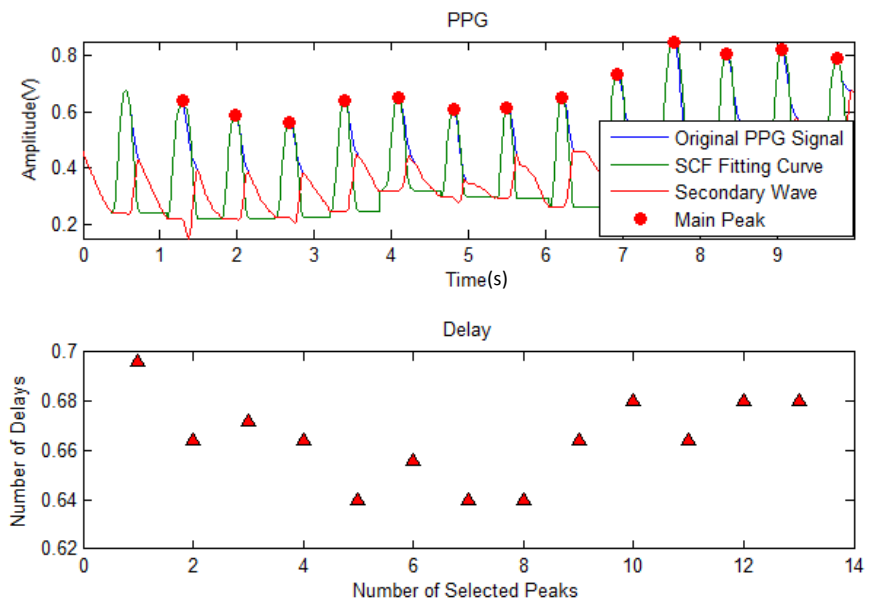

Figure 5.6: Adjusted PPG signal and adjusted PTT by SCF 

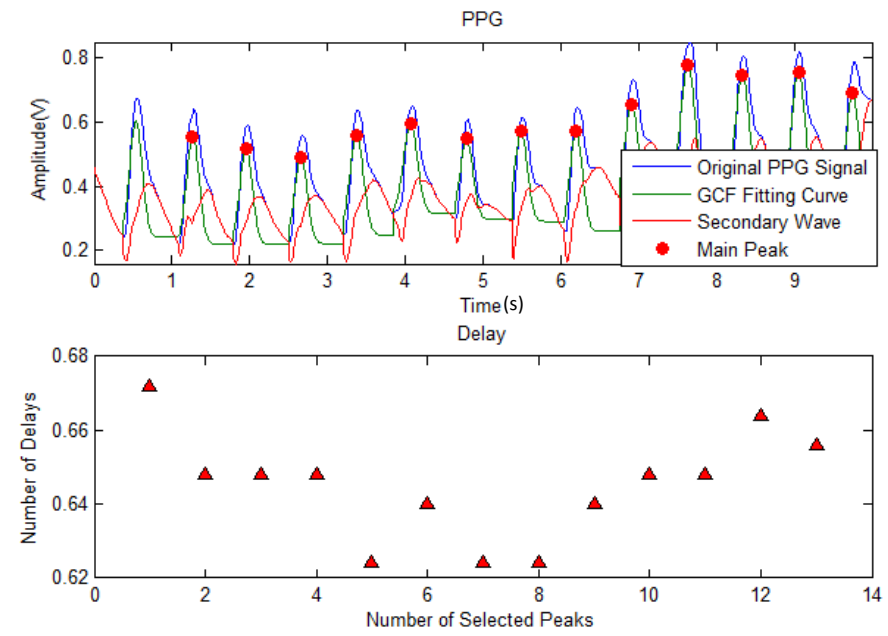

Figure 5.7: Adjusted PPG Signal and adjusted PTT by GCF
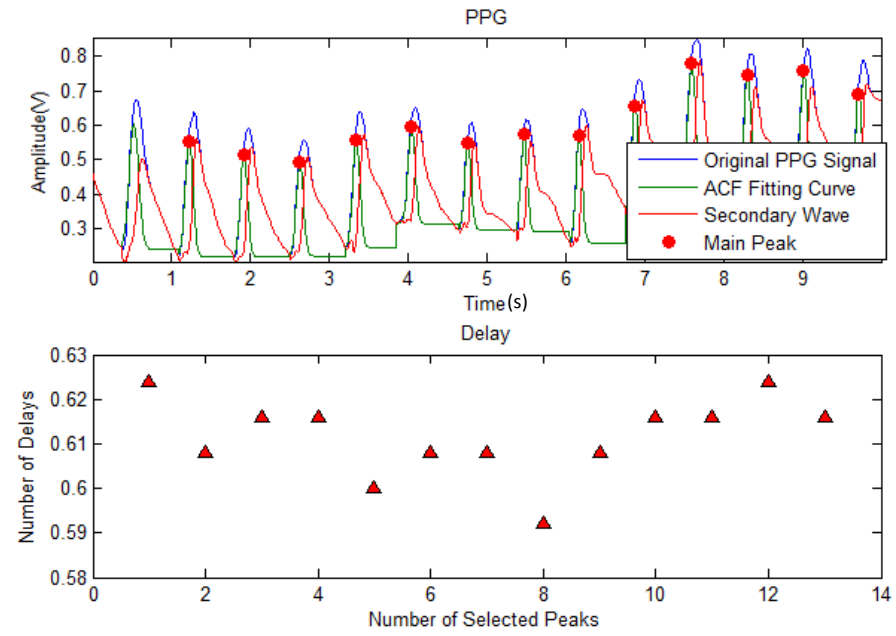

Figure 5.8: Adjusted PPG Signal and adjusted PTT by ACF

The SBP and DBP for Figure 5.6, Figure 5.7, and Figure 5.8 are around $120 \mathrm{mmHg}$ and $60 \mathrm{mmHg}$, respectively. As mentioned before, the adjusted PTT by using the SCF method is actually the same as the original PTT calculated from the original PPG. This can be used to evaluate the effect of the peak shifting phenomenon. From the plots we can see that the variation of the delay after employing GCF and ACF is smaller than the original 
PTT, which demonstrates that the adjusted PTT is more specific for the certain value of the arterial blood pressure.

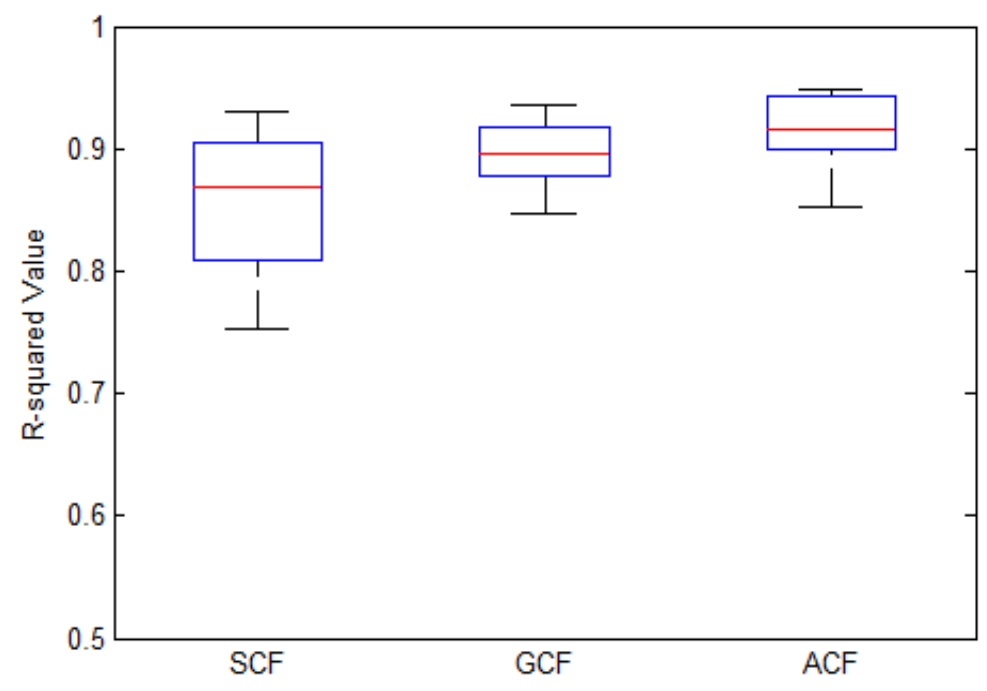

Figure 5.9: R-squared values between the adjusted PTT and the blood pressure by using SCF, GCF, and ACF detection methods

Figure 5.9 shows the R-squared values between the adjusted PTT signal and the blood pressure when using different secondary peak detection methods. R-squared value indicates how much the change in one variable can be explained by the change of the other variable, no matter whether their relationship is linear or nonlinear. It is obvious that the ACF method has better performance than the SCF method. So the reduction of the peak shifting problem and the detection of the secondary wave of the PPG signal are very necessary and important in using PTT for blood pressure measurement.

The characteristics of the arterial blood pressure and the PTT are different from subject to subject. The measurement of blood pressure, ECG, and PPG signals are subjectdependent. It is necessary to build the distinct relationship between the arterial blood 
pressure and the PTT for each patient. So in this part, the calibration procedure of the PTT to the blood pressure is considered.

If the relationship between the blood pressure and the PTT is assumed as linear, then at least two calibration points have to be taken into account and at least $15 \mathrm{mmHg}$ differences [11] of the blood pressure between the calibration points in order to get reliable accuracy. Each patient's record used in the simulations has twenty segments, selected to guarantee that the range of the arterial blood pressure varies from $70 \mathrm{mmHg}$ to $160 \mathrm{mmHg}$. Ten segments are used for individual calibration and the other ten segments are used for estimation.
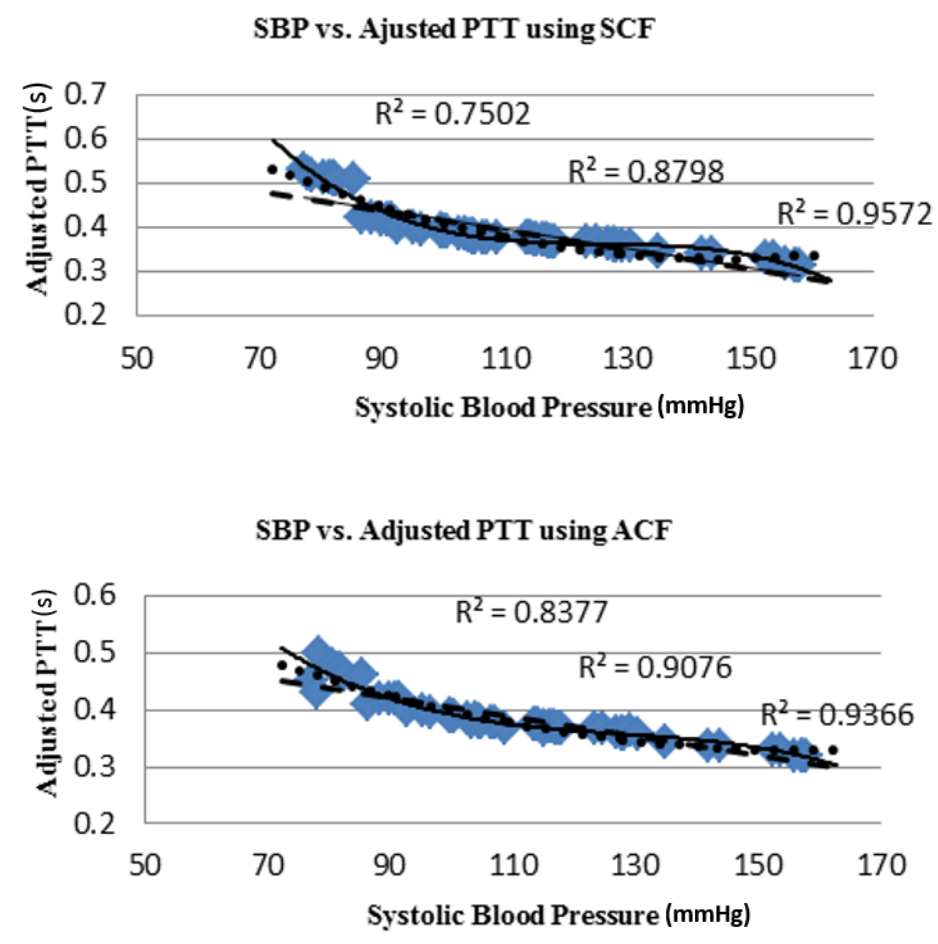

Figure 5.10: Scatter plot for SBP versus adjusted PTT and different regression curves

Figure 5.10 depicts the scatter plot for SBP versus the adjusted PTT by using different secondary peak detection methods. The linear (dash line), quadric (dot line), and cubic 
(solid line) regression curves are drawn. The regression curves and the R-square values show that the ACF method can provide better adjusted PTT, which has a higher correlation with the arterial blood pressure, especially when using linear regression curve.

In order to further illustrate the consistence between the proposed methods and the clinical data, Figure 5.11 shows the Bland-Altman plots under different regression methods. With more regression calculation, the cubic regression curve fits the data points better than the other two and the peak shifting impact can be further reduced.

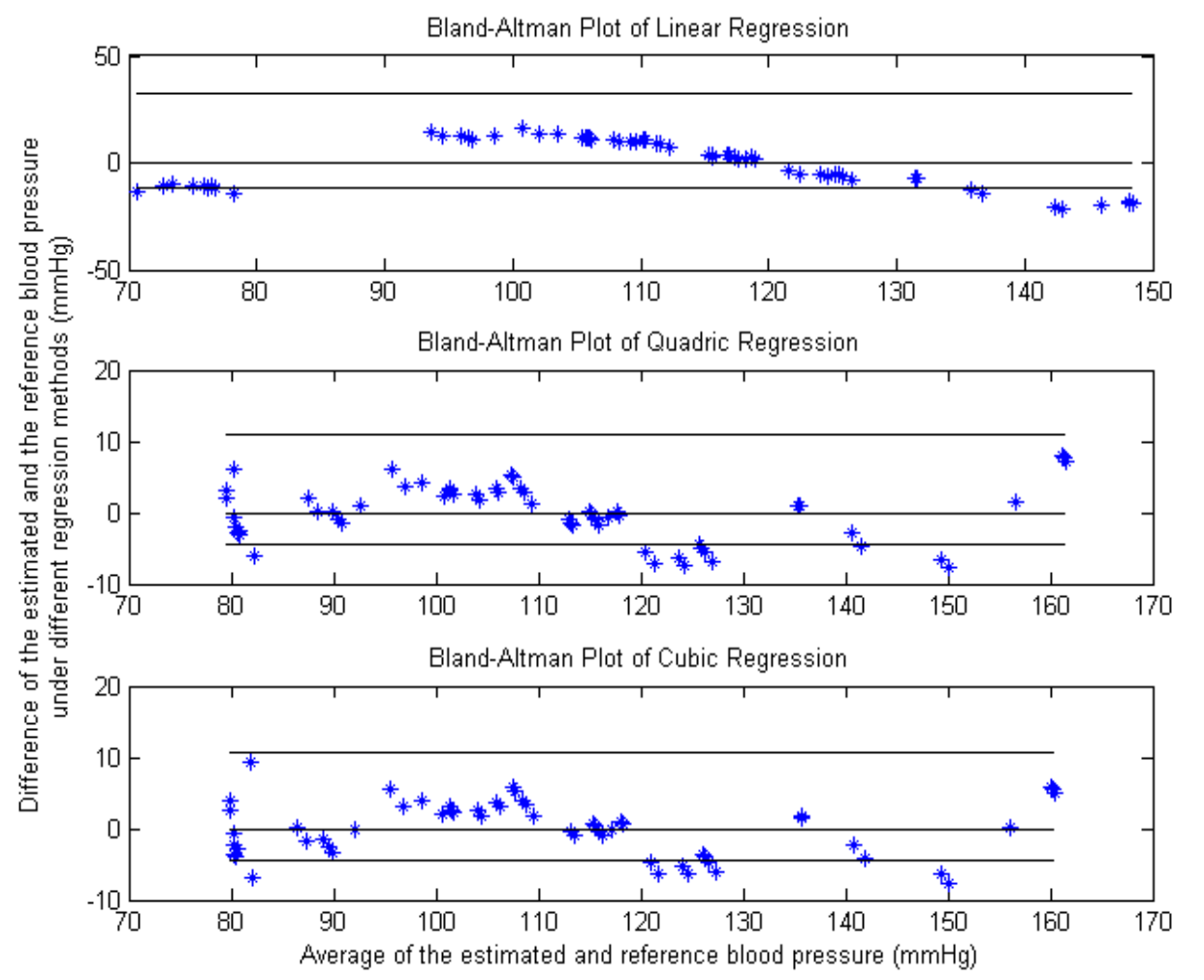

Figure 5.11: The Bland-Altman plot for the estimated and the reference blood pressure under different regression methods

The performance of the blood pressure estimation is depicted in terms of mean error (ME) and standard deviation of error (SDE). Since the objective is to see how close our 
estimated blood pressure is to the measured blood pressure, we are applying ME or mean absolute error (MAE) as criteria. Table 5.3 lists, in detail, the values of ME and SDE for different secondary peak detection methods for patient \#3302803 as an example. The ACF method has smaller ME and SDE than the other two methods, which means the estimated blood pressure obtained from ACF has smaller bias and error variance.

Table 5.3: Mean error and standard deviation of different secondary wave detection methods for patient $\# 3302803$

\begin{tabular}{cccc}
\hline Performance & SCF & GCF & ACF \\
\hline $\mathrm{ME}(\mathrm{mmHg})$ & 3.54 & 3.28 & 3.06 \\
$\mathrm{SDE}(\mathrm{mmHg})$ & 4.35 & 3.97 & 3.69 \\
& & & \\
\hline
\end{tabular}

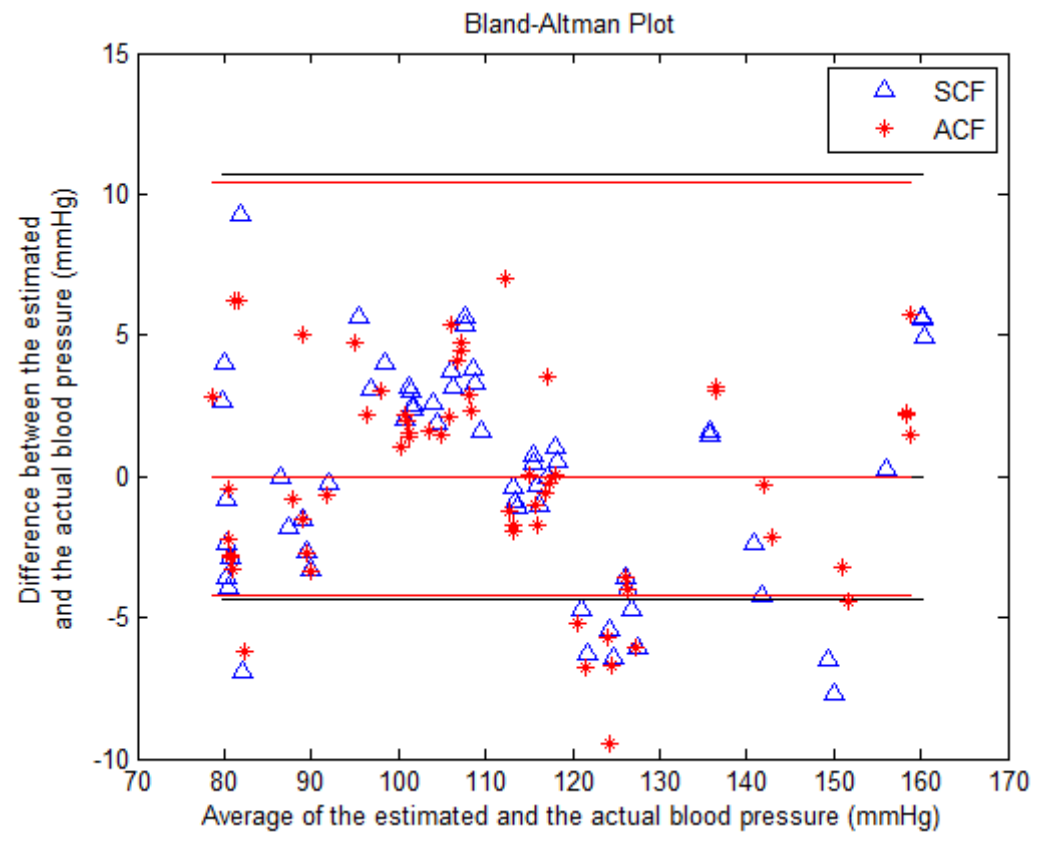

Figure 5.12: The Bland-Altman plot for the estimated and the actual blood pressure by SCF and ACF methods 
Figure 5.12 displays the Bland-Altman plot of the estimated SBP of the proposed methods versus the actual blood pressure. It shows the agreement between the predicted and the actual blood pressure on average. The blue triangles and the red asterisks represent the performance of the SCF and ACF methods, respectively. We can see that the majority of the points locate within the limits of the agreement. The curve obtained by the SCF method can predict the blood pressure within $3.54 \pm 4.35 \mathrm{mmHg}$ of the reference, while for the ACF method the value is $3.06 \pm 3.69 \mathrm{mmHg}$. This demonstrates that the estimation of the blood pressure can be improved by reducing the dicrotic notch impact and detecting the secondary peak of the original PPG signal.

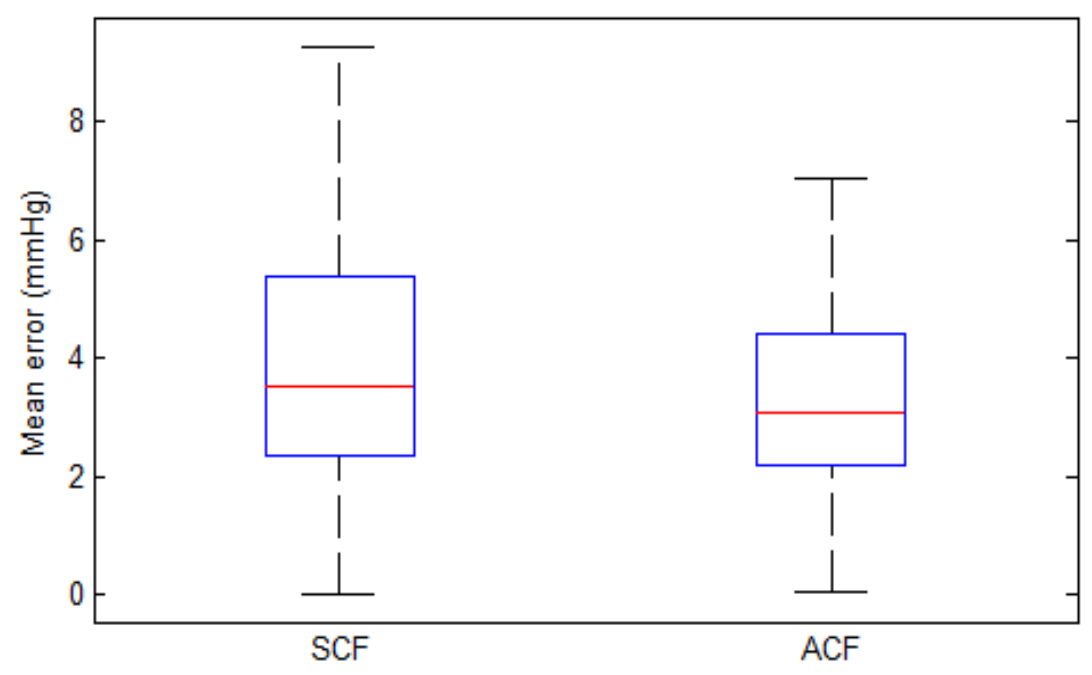

Figure 5.13: Boxplot of the average ME for the systolic blood pressure estimation by SCF and ACF methods

To better demonstrate how the secondary peak detection can improve the blood pressure estimation results, Figure 5.13 shows the boxplot of the ME values of using SCF and ACF. In Figure 5.13, the red central mark is the mean value of the error, the edges of the box are the 25th and 75th percentiles, and the black lines are the extreme points. We 
can see that the ME of using the SCF method can be as large as $9.27 \mathrm{mmHg}$, while the maximum value of the ME of using the ACF method is $7.04 \mathrm{mmHg}$. The values of mean errors for SCF and ACF methods are $3.54 \mathrm{mmHg}$ and $3.06 \mathrm{mmHg}$, respectively. Therefore, the performance of the $\mathrm{ACF}$ is better than the SCF in both mean and variance of errors.

\subsection{Summary and Conclusion}

In this chapter, the correlation and the linear regression, the quadric regression, and the cubic regression models were used to evaluate the relationship between the PTT and different types of arterial blood pressure. Simultaneous neat ABP, ECG, and PPG signals were extracted from the MIMICII database.

The correlation between the adjusted PTT and the arterial blood pressure was calculated. Linear regression, quadric regression, and cubic regression models were used to evaluate the relationship between the adjusted PTT and the SBP. Simulation results demonstrated that the GCF and ACF can resolve the peak shifting problem and the adjusted PTT had higher correlation with the blood pressure than the original PTT without an observable dicrotic notch. Among the three regression models, the cubic regression method fitted the adjusted PTT versus blood pressure data points best. This investigation verified that the adjusted PTT can be used to determine the blood pressure continuously and noninvasively without the usage of a cuff, which is convenient and very useful for selfmonitoring patients. 


\section{Chapter 6}

\section{Wrist Pulse Measurement and Analysis Using Eulerian Video Magnification Framework}

\subsection{Introduction}

Pulse signal diagnosis has been used in traditional Chinese medicine for thousands of years. It has been proved that wrist pulse signals contain vital information on a person's health status and can reflect the pathological changes [174]. Clinical experiments demonstrate that the shape of the wrist pulse signals has high correlation with the characteristics of the blood flow [175].

As one of the four traditional diagnostic methods, pulse diagnosis of palpation is more important, but more difficult than inspection, auscultation, and questioning [176]. The physicians use three fingertips on the radial artery of the patient's wrist to assess the patient's health condition. Three locations, named "cun", "guan", and "chi”, are chosen [177]. So the diagnosis results mainly depend on the experience and the skills of the physicians, which means different physicians may have different results for one patient's 
wrist pulse. To improve the accuracy of the wrist pulse detection, computerized wrist pulse signal measurement is needed to objectify the ancient diagnostic technique.

Computerized wrist pulse signal measurement and analysis usually has three stages [178]. The first stage is data collection. The second and third stages are feature extraction and pattern classification. Former research has proposed many models and algorithms to extract and classify the feature parameters from the wrist pulse signals, such as Gaussian model, information fusion technology, and clustering technique [179][180][181][182] [183]. The detection and location of partially overlapped peaks in a multi-component signal was facilitated by mathematical differentiation [184].

Usually, wrist pulse signals are collected by different types of sensors or probes. A Doppler ultrasound blood analyzer was applied in [185]. Piezoelectric film sensors were employed to replace the three fingertips to detect on "cun", "guan", and "chi" [186]. The use of sensors or probes can be heavily interfered by blood flow in the wrist and may be uncomfortable for patients, then affect the measurement accuracy. And this is not convenient for self-monitoring.

A novel method called Eulerian video magnification (EVM) is recently introduced to reveal the invisible subtle variations in videos [146]. The system uses a standard video sequence as the input. Then spatial decomposition and temporal filtering are applied to the frames. The filtered signal is then amplified to reveal the subtle changes, either in the color or the motion changes on the skin caused by the wrist pulse when blood flow passes. With this technique, the wrist pulse signal, which cannot be seen by the naked eye, can be measured non-intrusively. 
In this chapter, the EVM method is first introduced to measure and analyze wrist pulse signals. The waveform of the wrist pulse signal is collected from the video and the characteristics of the pulse are extracted by applying two-Gaussian curve modeling method. This can open the possibility on using a video-based non-intrusive way to measure wrist pulse signal.

\subsection{Overview of the Eulerian Video Magnification Framework}

The purpose of EVM is to overcome the limitation of human visual system and reveal more informative signals, which are invisible to the naked eye. The framework of EVM is first brought up by Hao-yu $\mathrm{Wu}$ [146] in 2012. The basic approach was to observe the series of color values in time scale at any spatial location, say pixel, and then amplify the change in a certain temporal-frequency band [188].

\subsubsection{Framework of Eulerian Video Magnification}

Figure 6.1 shows the main framework of EVM in measuring the wrist pulse signal. The video is processed frame by frame and the subtle variations in the video are emphasized by the spatial and temporal processing, which involved several steps and can be described as follows:

Step 1) Collect the standard input video that may contain informative color changes on the wrist.

Step 2) Apply a full Laplacian pyramid [189], which is a set of band-pass filters, to decompose the standard video sequence into different spatial frequency bands. Different magnification might be made on these bands because of different signal-tonoise ratio (SNR) or unsuitable spatial frequency for linear approximation. 


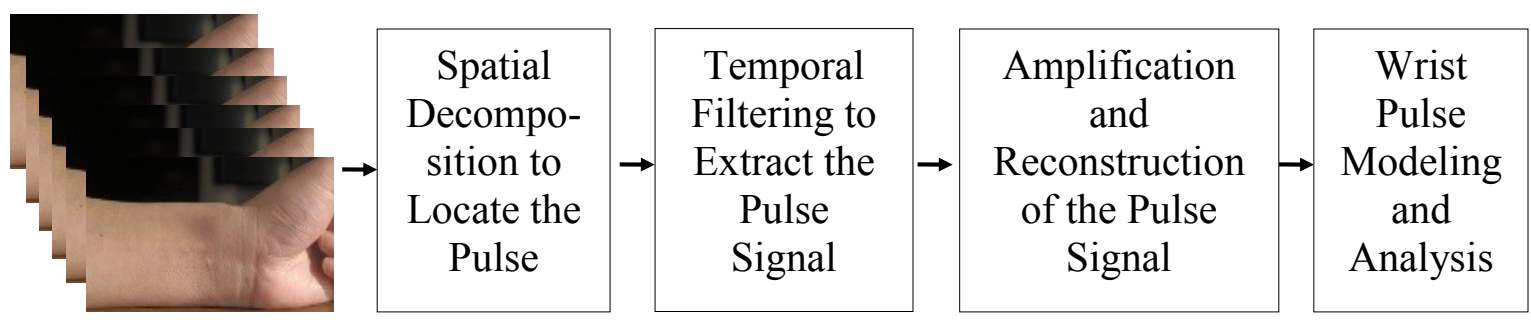

Figure 6.1: Framework of Eulerian video magnification method

The Laplacian pyramid uses a Laplacian transform to process video frames. It decomposes the original image into a hierarchy of images and each level of the decomposed image corresponds to a different frequency band. The Laplacian pyramid of image can be described as:

$$
L_{i}(P)=G_{i}-\operatorname{EXPAND}\left(G_{i+1}\right)
$$

where $G_{i}$ represents the different levels in the adjacent low-pass images of the Gaussian pyramid. The reversal of the process can reconstruct the original image.

Step 3) Perform the temporal processing on each of the spatial bands. During this step, different types of band-pass filters will be chosen according to the frequency band of interest. The temporal filtering process amplifies not only the color variation, but also the low amplitude motion. It keeps the same for all spatial levels and for all pixels within each frequency level.

Step 4) Employ a magnification factor $\alpha$ to multiply the extracted band-pass signal. The value of $\alpha$ is specified by different applications and the boundary of $\alpha$ is determined by the video motion step $\delta$ and the image structure spatial wavelength $\lambda$. The equation is given by

$$
(1+\alpha) \times \delta \leq \lambda / 8
$$


where $\lambda=2 \pi / \omega$ and $\omega$ represents the specific spatial frequency.

Step 5) Select a spatial cut-off frequency. $\alpha$ is attenuated beyond this frequency as shown in Figure 6.2.

Step 6) Reconstruct the signal by adding the magnified signal to the original one and collapsing the spatial pyramid.

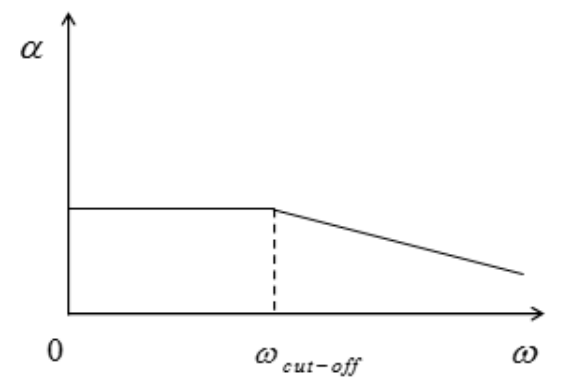

(a)

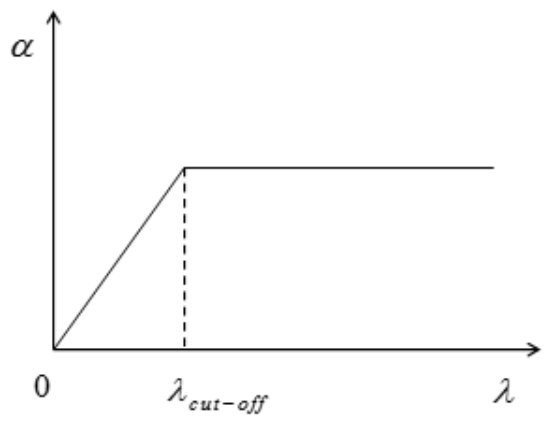

(b)

Figure 6.2: Bound of the magnification factor $\alpha$

Figure 6.2 (a) shows the relationship between the magnification factor $\alpha$ and the spatial frequency $\omega$. Figure 6.2 (b) illustrates the relationship between $\alpha$ and the spatial wavelength $\lambda$. The area of lower spatial frequencies is magnified, which reveals the subtle variations of the video. 


\subsubsection{Eulerian Color Magnification}

As mentioned above, the temporal filtering procedure cannot only amplify the color variation but also enhance the low amplitude motion. So basically, there are two types of EVM, Eulerian color magnification and Eulerian motion magnification.

Hao-yu $\mathrm{Wu}$ and his colleagues have shown several perfect examples of both magnification methods on their research website [190]. According to each magnification method, the choice of temporal filter is different. A narrow band-pass filter is preferred for Eulerian color magnification while a broad band-pass filter is more suitable for Eulerian motion magnification.

The color component of EVM method is applied in this chapter. The low spatialfrequency changes are amplified, so the periodic color variation caused by blood pulse when blood flows through different locations in the wrist can be visualized.
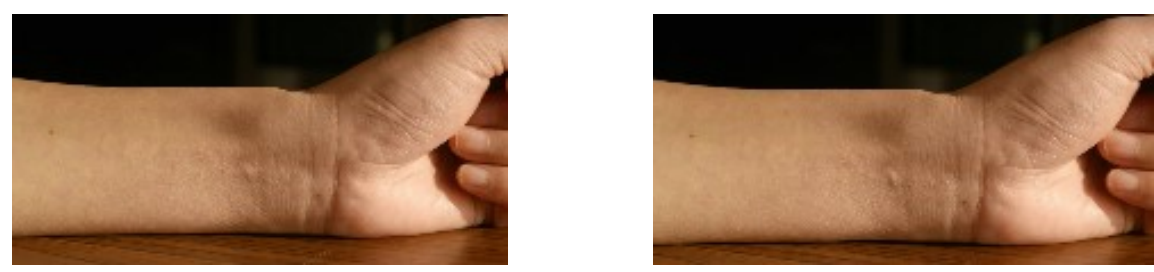

(a) Input
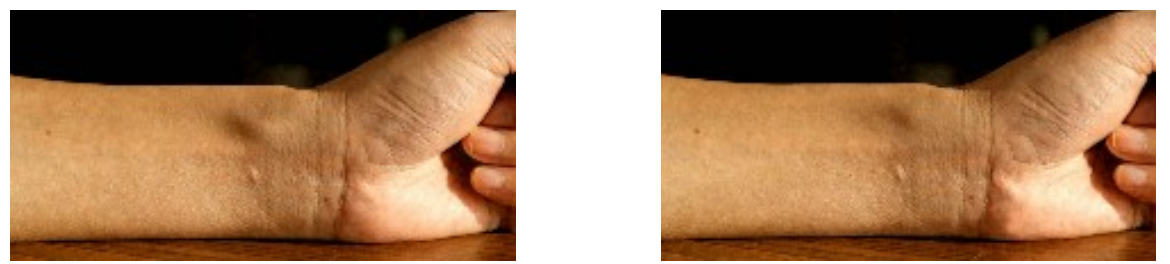

(b) Output

Figure 6.3: Two frames of the wrist video sequence. The output frames display the subtle changes on the wrist 
Figure 6.3 (a) displays two frames from the input video sequence. No color changes are visible in the input frames. The outputs of the same two frames in Figure 6.3 (a) are illustrated in Figure 6.3 (b). It can be observed that the variation of the wrist pulse is visualized after amplifying the reconstructed signal. Comparing to the input frames, the output frame in the left figure is stronger, while the output frame in the right figure is weaker.

The three-dimensional surface plot of one frame after the spatial decomposition and temporal filtering is shown in Figure 6.4. Color is proportional to the height of the surface.

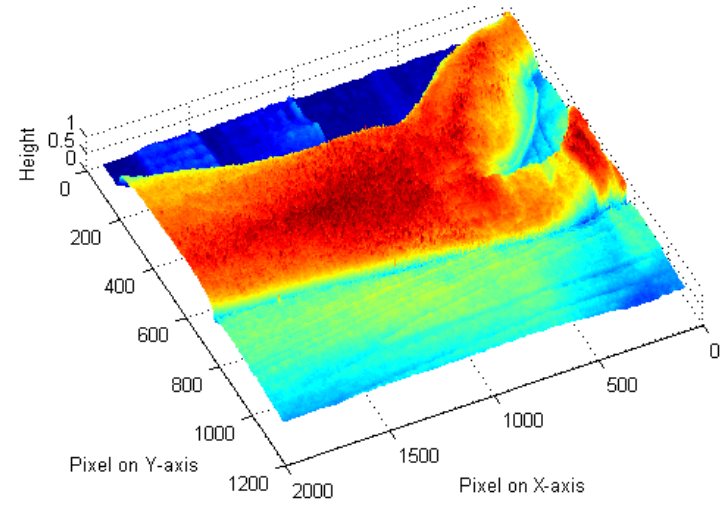

Figure 6.4: Three-dimensional surface plot of one frame of the input video

\subsection{Wrist Pulse Signal Acquisition}

In the traditional Chinese medicine, the pulse diagnosis of palpation is realized by physicians placing three fingertips over the radial artery of a patient's wrist. The wrist pulse contains useful information about the inside body and can reflect the type of disease [187].

In this chapter, a video camera is used to capture a standard video sequence of the wrist. The EVM method is employed to detect, non-intrusively, the wrist pulse signals. The data was collected according to the following three steps: locate the rough area in the wrist, 
obtain the most significant signal by measuring around the rough area, and record the wrist pulse signal.

Instead of placing fingertip over the radial artery, the region of location "guan" on the wrist is selected for the contact-free measurement. Figure 6.5 shows the location chosen to measure the wrist pulse signal and its corresponding waveform obtained by the EVM method.
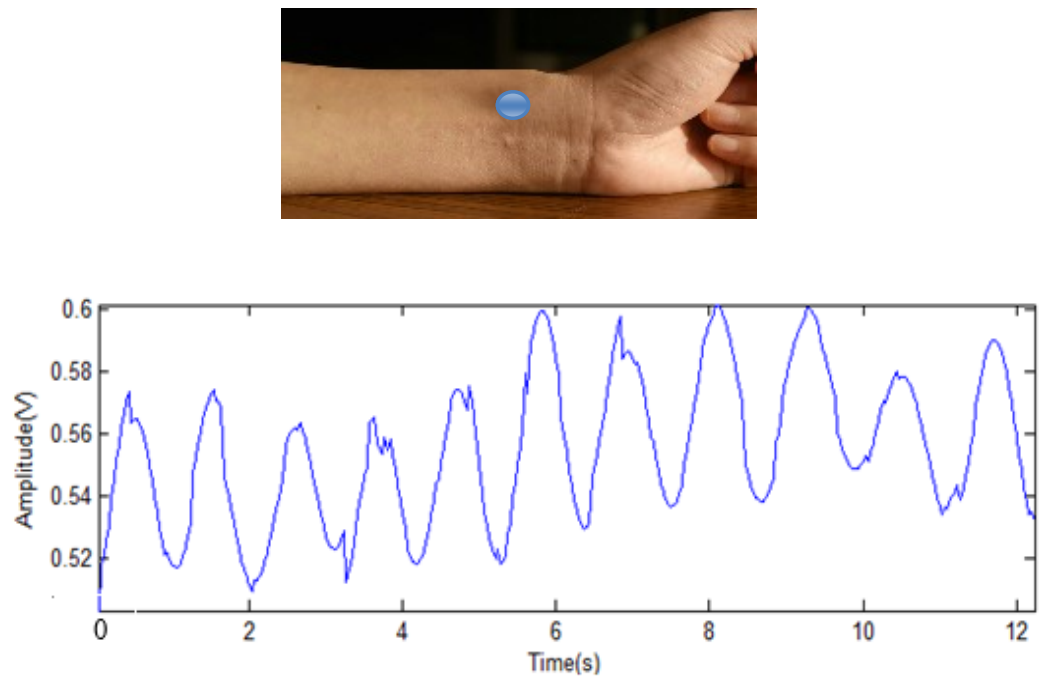

Figure 6.5: The selected location for wrist pulse signal measurement and its corresponding waveform

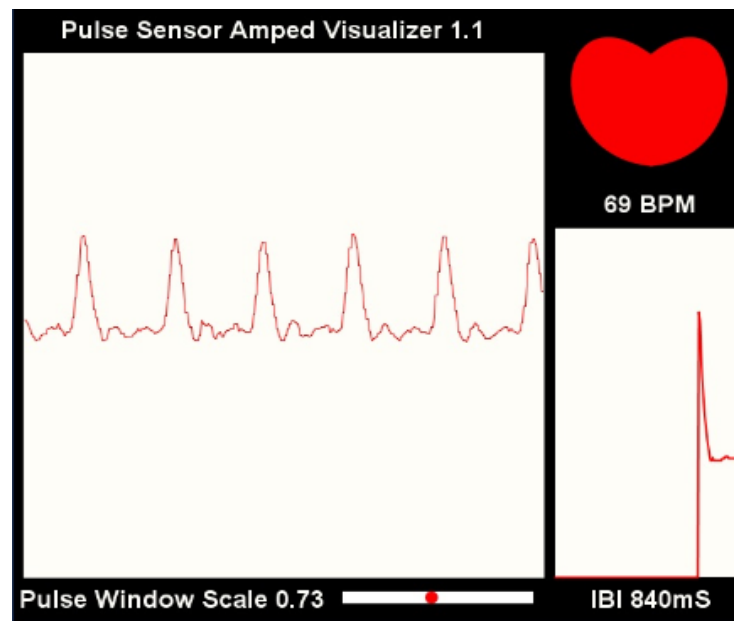

Figure 6.6: Finger PPG signal measured by Arduino board and PulseSensor 
Figure 6.6 is the measured finger PPG pulse by the Arduino board and the PulseSensor. It is measured at the same time when the video is taken. This can guarantee the synchronism of the detected wrist pulse signal and the finger PPG signal. IBI is the inter-beat interval.

\subsection{Wrist Pulse and PPG Pulse Modeling}

To observe the features of the wrist pulse signal, one period waveform is selected. There are two main peaks in a single period wrist pulse signal, the primary peak and the secondary peak. The secondary wave is formed by the reflection of the blood flow on the periphery of the arterial system [181].

Given the characteristics of the pulse signal, a Gaussian curve can be used to model it. In this paper, we apply the two-Gaussian curve modeling method to analyze the pulse signals. After setting the initial values of the coefficients of the Gaussian curves, the fitted model was determined by the combination of two methods: the nonlinear least squares algorithm and the "Trust-Region" fitting algorithm. The Gaussian equation generally involves three parameters, the amplitude $(A)$, the mean $(\mu)$, and the standard deviation $(\sigma)$ [191]. The equation used for describing the fitted model is as follows:

$$
\mathrm{G}(\mathrm{x})=\sum_{\mathrm{j}=1}^{2} \mathrm{~A}_{\mathrm{j}} \exp \left(-\frac{\left(x-\mu_{j}\right)^{2}}{2 \sigma_{j}^{2}}\right)
$$

where $x$ is the original pulse signal and is the sum of the two Gaussian curves used to model the pulse signal.

When applying the two-Gaussian curve modeling method, the fitted model was determined by the combination of the nonlinear least squares algorithm and the "TrustRegion” fitting algorithm. 


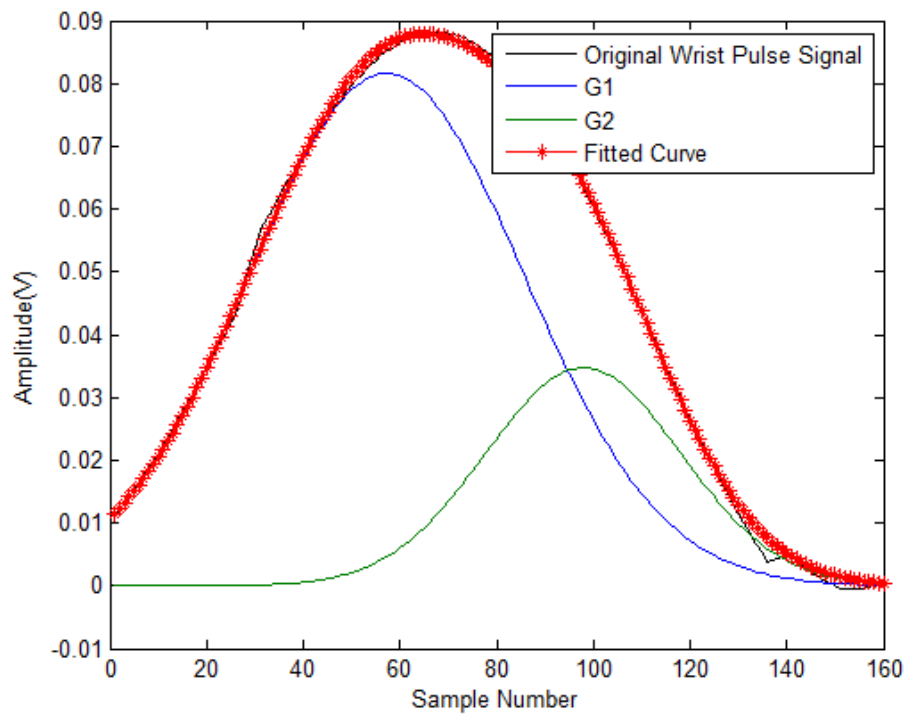

Figure 6.7: Two-Gaussian curve modeling for the wrist pulse signal detected by Eulerian video magnification method

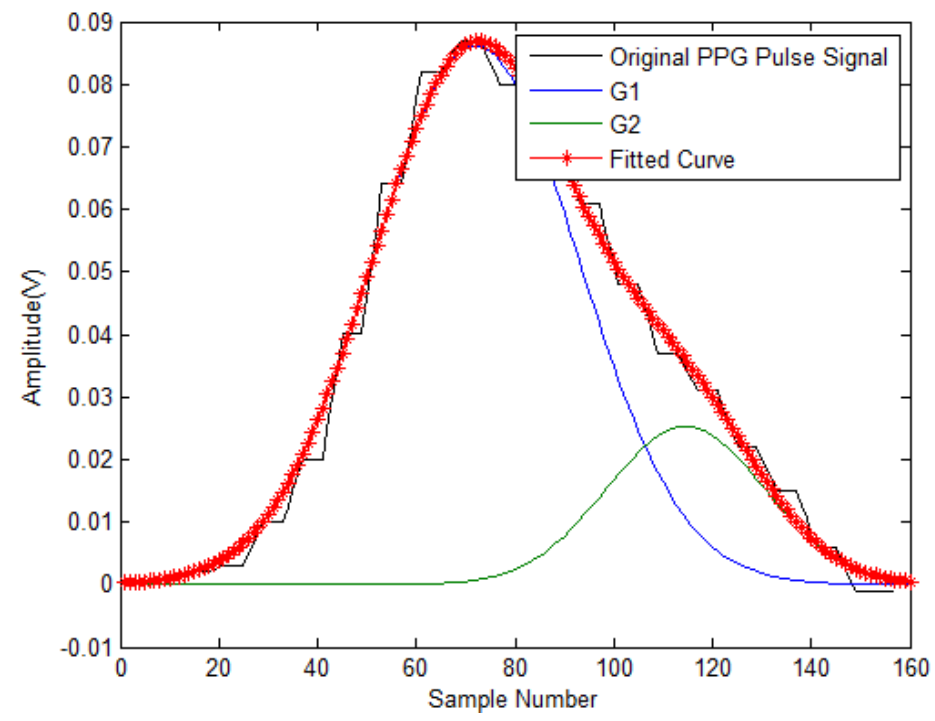

Figure 6.8: Two-Gaussian curve modeling for the PPG pulse signal measured by the Arduino board and the PulseSensor

Figure 6.7 and Figure 6.8 show an example where the two-Gaussian curve modeling method was applied on the detected wrist pulse signal and on the PPG pulse signal, respectively. In both figures, the black curve is the measured pulse signal. The red star 
curve stands for the fitted curve. The blue and green curves represent the two modeling Gaussian curves, respectively.

\subsection{Characteristic Parameter Comparison}

The standard video camera used in the experiments has one million effective pixels. The video was compressed to a resolution of $640 \times 360$ at 30 frames per second. The spatial and temporal processing was done by MATLAB. To evaluate the proposed system, the Arduino board and the PulseSensors [192] were used to simultaneously measure the corresponding finger PPG pulse.

The pulse signals contain many characteristic parameters. Each parameter happens for a reason and reflects the condition of the cardiovascular condition. To verify the proposed system, we calculate the average of the following parameters of the two fitted Gaussian curves for the detected wrist pulse signal and the PPG pulse signal: the ratio of the amplitude, the ratio of the phase shift, and the ratio of the width. The values of different characteristic parameters are given in Table 6.1.

Table 6.1: Comparison of the values of different characteristic parameters

\begin{tabular}{ccc}
\hline Average & Wrist Pulse & Finger Pulse \\
\hline$A 1 / A 2$ & 2.601 & 2.590 \\
$\tau 1 / \tau 2$ & 0.588 & 0.603 \\
$w 1 / w 2$ & 1.424 & 1.295 \\
$T(s)$ & 0.869 & 0.833 \\
\hline
\end{tabular}


In Table 6.1, $A 1 / A 2$ is the average ratio of the amplitude, $\tau 1 / \tau 2$ is the average ratio of the phase shift, $w 1 / w 2$ is the average ratio of the width of the two Gaussian curves, and $T$ is the pulse period.

From the selected characteristic parameter values shown in Table 6.1, we can see that the difference between the two sets of fitted Gaussian curves are small. So the two sets of fitted Gaussian curves reflect similar cardiovascular conditions.

Consider the PPG pulse signal measured by the Arduino board and the PulseSensor as the reference signal. By modeling the wrist pulse signal being detected through the video-based technique, the characteristic parameters containing important information on health condition can be obtained. The proposed system has high potentiality to be employed for wrist pulse signal measurement.

\subsection{Summary and Conclusion}

The measurement and analysis of the wrist pulse signal has always been an important tool to diagnose health status for thousands of years. The waveforms of the wrist pulse signal can be used to track the pathologic changes of a subject's health condition. With the development of computer technology, computerized wrist pulse signal collection and classification have played a major role to conquer the difficulties existing in the traditional Chinese medicine.

In this chapter, a novel non-intrusive contact-free system based on the EVM method was proposed on observing blood flow through the wrist and collecting the wrist pulse signal. Location "guan", which was used in the traditional palpation technique, was selected in the experiment. To verify the proposed system, the Arduino board and the 
PulseSensor were applied to measure the finger PPG pulse signal at the same time. In the future, the EVM method may be implemented on the Arduino board to detect the pulse signals. The two-Gaussian curve modeling method was employed on fitting the wrist pulse signal and the finger pulse signal for analyzing the characteristics of these two signals.

Preliminary results showed high correlation between the wrist pulse signal and the PPG pulse signal. The heart rate obtained through the two pulse signals was the same. The EVM method was demonstrated that can be used as a reliable method to collect the wrist pulse signal since the PPG signal had been used in estimating the arterial blood pressure. The wrist pulse signal detected by the EVM method can be considered having the potential of predicting cardiovascular events. 


\section{Chapter 7}

\section{PTT Measurement Using Eulerian Video Magnification Framework}

\subsection{Introduction}

Recent advances in sensor technology and mobile computing are now enabling practical non-intrusive approaches to measure vital signs and some biological signals. A nonintrusive load monitoring system was proposed to monitor the activities of daily living within a smart home [193]. Furthermore, most smart phones are now equipped with high resolution cameras and powerful processors that can reliably measure these signals. One of the signals of interest is the PTT that is often correlated with changes in the blood pressure and stress level [194][195][196]. One technique for measuring PTT is based on measuring the ECG signal using leads attached to the chest and measuring the PPG signal from a finger [197][198][199].

In Chapter 6, the Eulerian video magnification (EVM) framework was applied on measuring and analyzing the wrist pulse signal, which is one area of interest on the human body. This chapter the EVM is introduced to detect the color changes on two different areas 
on the human body to measure the PTT non-intrusively. The proposed approach uses a video camera to capture a standard video sequence of the subject. After applying spatial decomposition and temporal filtering to the frames, the filtered signal is then amplified to reveal the subtle changing, like the color changing on different spots caused by the blood pulse. Two spots, the wrist and the neck, were selected to measure the PTT.

To verify the performance and practicability of the proposed system, the measured PTT were compared with the time difference detected using a conventional technique based on two PulseSensors and the Arduino board. Ten subjects were studied under three statuses: climbing stairs, five minutes rest after climbing stairs, and twenty minutes rest after climbing stairs. The experimental results show that the PTT measured by the EVM framework is highly correlated with the PTT detected by pulse sensors, demonstrating that the proposed approach has the potential to be used for health-care monitoring.

\subsection{Color Variation Detection}

Eulerian color magnification is used in this study and low spatial frequency changes are emphasized since we are looking into the periodic color variations caused by the blood pulse when blood flows through different areas.

Figure 7.1 (a) shows two frames from the original video sequence revealing the pulse. From the input of the two frames, no color changing is visible. Figure 7.1 (b) is the output of the same two fames shown in Figure 7.1 (a). It can be obviously seen that the variation

of the color is visualized after the amplification. When the blood pulse reaches the certain spot, the color of the skin at that spot will turn darker (redder in Wu's work). When the blood pulse is far away, the color will get lighter. 

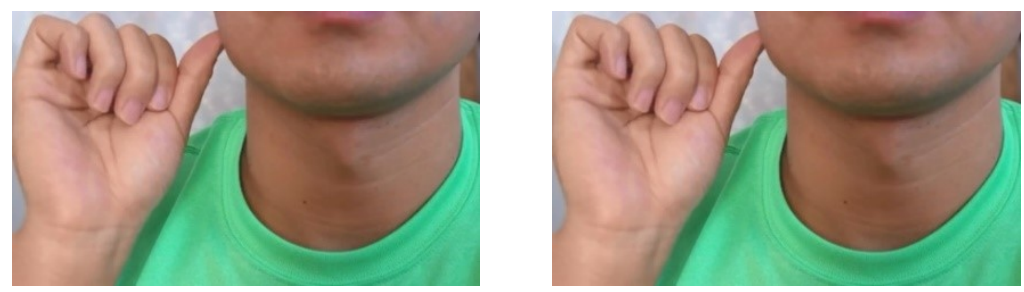

(a) Input
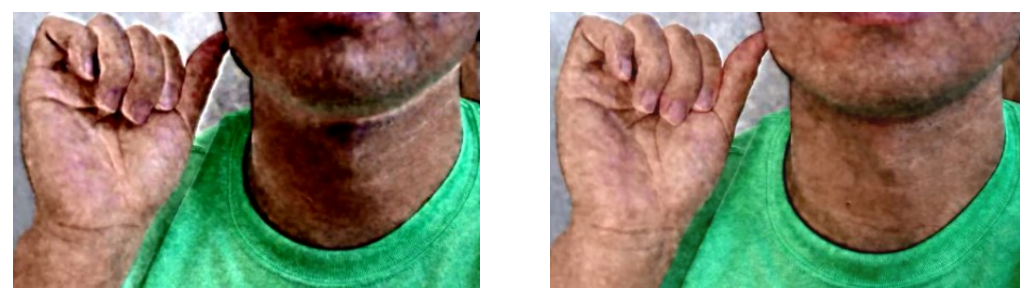

(b) Output

Figure 7.1: Two frames from one of the original video sequence showing the color changing on the wrist and neck caused by blood pulse

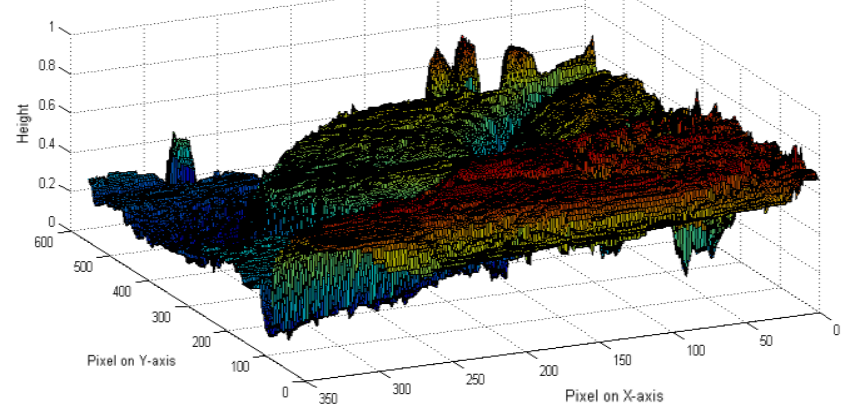

(a)

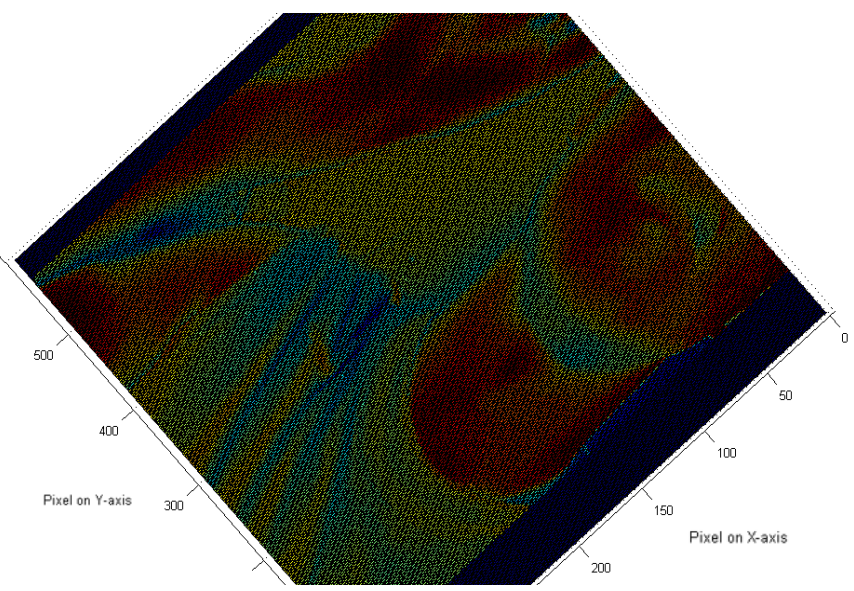

(b)

Figure 7.2: Three-dimensional surface plot of one frame 
Figure 7.2 shows an example of the three-dimensional surface plot of one frame after the spatial and temporal processing. Figure 7.2 (a) displays the overall front view of the selected frame. After rotating the 3D plot into a certain point of view, the RGB frame of the image in Figure 1 can be seen much more clearly in Figure 7.2 (b).

\subsection{PTT Extraction}

PTT is a convenient parameter for continuous monitoring and is highly related to pulse wave velocity [200], which is a very important electromechanical characteristic of biomedical signals. Literally, PTT is the propagation time delay between two points. The conventional way to measure PTT is to calculate the time interval between the peak of QRS-complex of ECG signal and the corresponding PPG measurement site.

In order to simplify the procedure of the PTT measurement for health-care monitoring, the Eulerian color magnification framework is introduced to realize a way to continuously and non-invasively measure PTT, furthermore, to estimate several biomedical characteristics.

Eulerian color magnification is used to amplify the color variation of the human skin caused by blood pulse. Figure 7.3 illustrates the two areas we are looking into in this chapter. One is on the wrist and the other one is on the neck. Obviously, the moments of blood flow reach these two areas are slightly different. The color variation can be revealed by Eulerian color magnification. Then the blood pulse can be captured to calculate the PTT. 


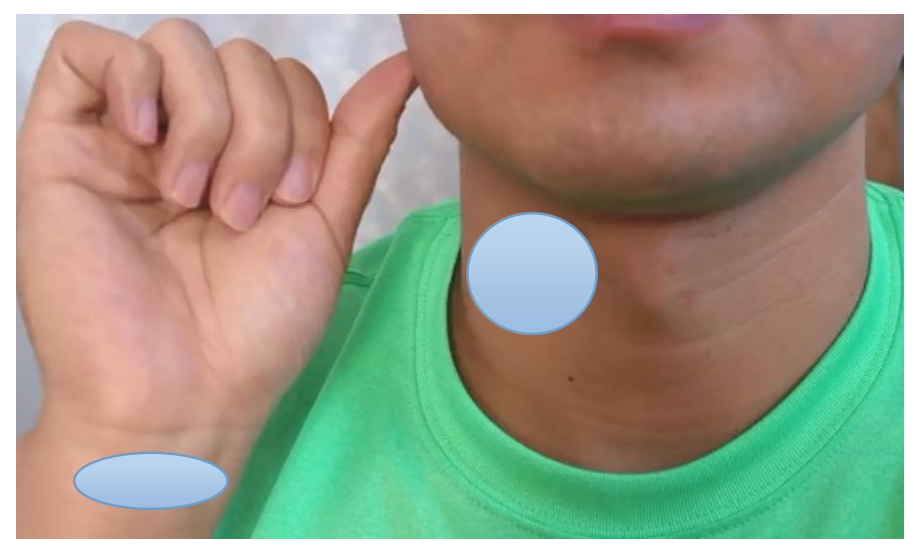

Figure 7.3: Selected pulse detection area of interest

The green curve and the black curve in Figure 7.4 are the amplified color variation on the wrist and the neck, respectively. After the amplification, the subtle changes of the color can be observed clearly and the blood pulse can be obtained easily. The red triangles and the blue triangles stand for the moment when the blood flow reaches the measurement sites. From these triangles not only the PTT can be calculated, but also the heart rate can be obtained. From Figure 7.4, it can be seen that in 18 seconds, there are 16 pulses occur on both sites of interest.

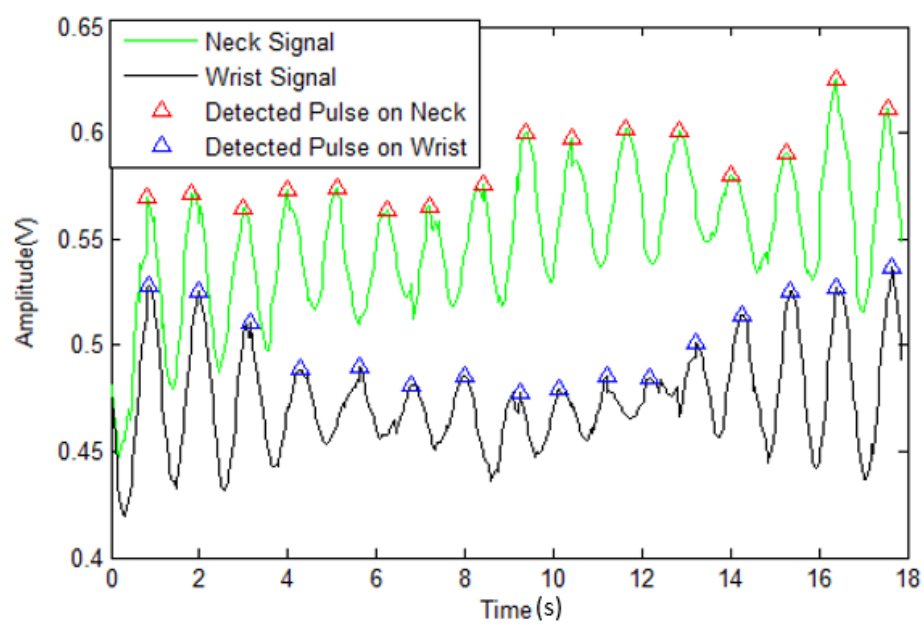

Figure 7.4: Pulse detection by using Eulerian color magnification on wrist and neck simultaneously 


\subsection{Experiments Using Eulerian Color Magnification}

Ten healthy subjects were tested in the experiments, with ages from 26 to 32 . A standard video camera was used to obtain the video sequences. The recorded videos have a frame resolution of $640 \times 360$ and a rate of 30 frames per second. The spatial and temporal processing was done by MATLAB. We used the second-order IIR band-pass filter to amplify the color variations, with low and high cut-off frequencies of $0.8 \mathrm{~Hz}$ and $2 \mathrm{~Hz}$, respectively. The magnitude response of the filter is shown in Figure 7.5. Table 7.1 shows the values of parameters used in Eulerian color magnification to generate the amplified output.

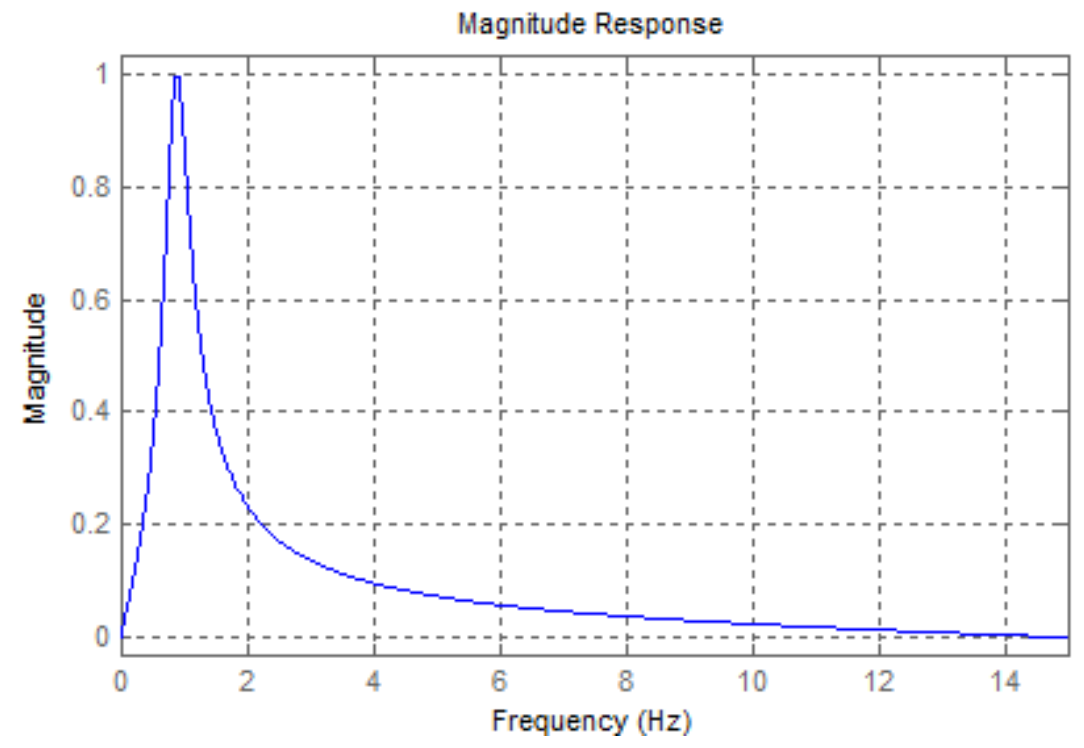

Figure 7.5: The temporal filter used to amplify the color variation 
Table 7.1: Values of parameters used in Eulerian color magnification to generate the

\begin{tabular}{cc}
\multicolumn{2}{c}{ amplified output } \\
\hline Parameters & Value \\
\hline Magnification factor $(\alpha)$ & 500 \\
Wavelength $(\lambda)$ & $0.6 \mathrm{~Hz}$ \\
Low frequency bound of the band-pass filter & $3 \mathrm{~Hz}$ \\
High frequency bound of the band-pass filter & $30 \mathrm{~Hz}$ \\
Frame rate & 0.1 \\
\hline
\end{tabular}

The video for each subject was recorded under three scenarios. One was recorded once the subject finishing climbing five flights of stairs. One was taken when the subject has had five minutes rest after climbing the stairs. And the third scenario was the subject has had half an hour rest after the stairs.

Normally, the color variations of human skin along with the blood pulse and the respiration are invisible to the naked eye. By applying Eulerian color magnification framework, the color changes can be detected and the amplified signal is very useful for vital biomedical signals' estimation and health-care monitoring. As shown in Figure 7.1, the color of the skin changes with the blood flow. By extracting the color signal and locating the peaks of the signal, we can determine both the heart rate and the PTT. The videos for the experiments were taken in an office room with incandescent lamp. Compared to the color changes on the skin, the changes of the light condition was insignificant and would not affect the detection of the pulse signals. 


\subsection{Evaluation}

We evaluated our proposed system by measuring the PTT using the Arduino board and the PulseSensor [192], shown in Figure 7.6. There are two pulse sensors, applying on the wrist and the neck, respectively.

Figure 7.7 is an example of the measured pulse signals from the wrist and the neck by PulseSensor and the Arduino board. The red curve represents the pulse signal from the neck and the green curve is the pulse signal from the wrist. IBI is the inter-beat interval.

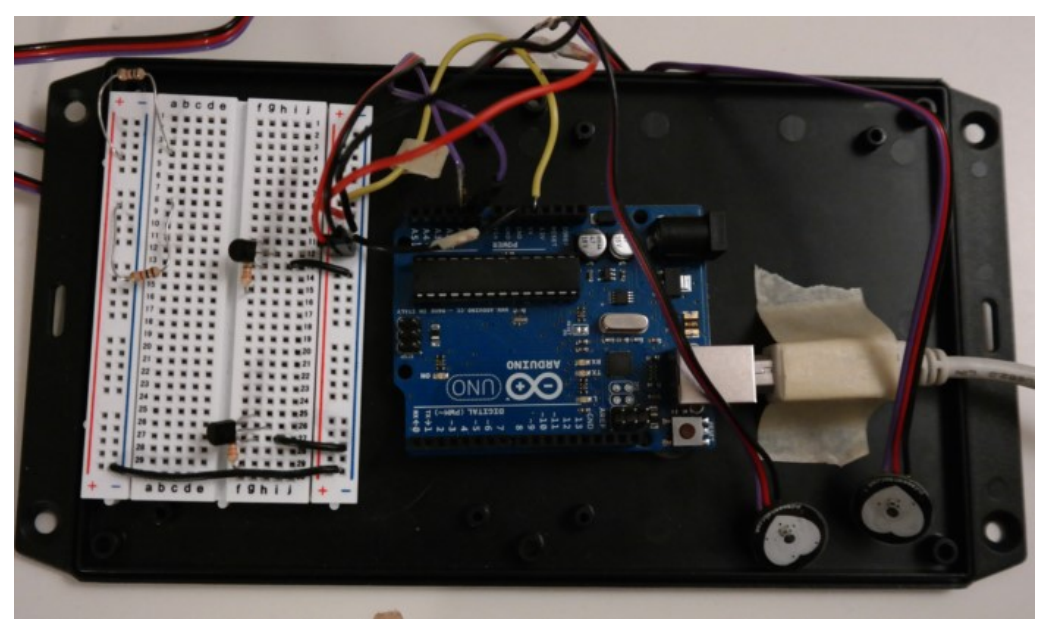

Figure 7.6: The Arduino board and the pulse detecting sensors

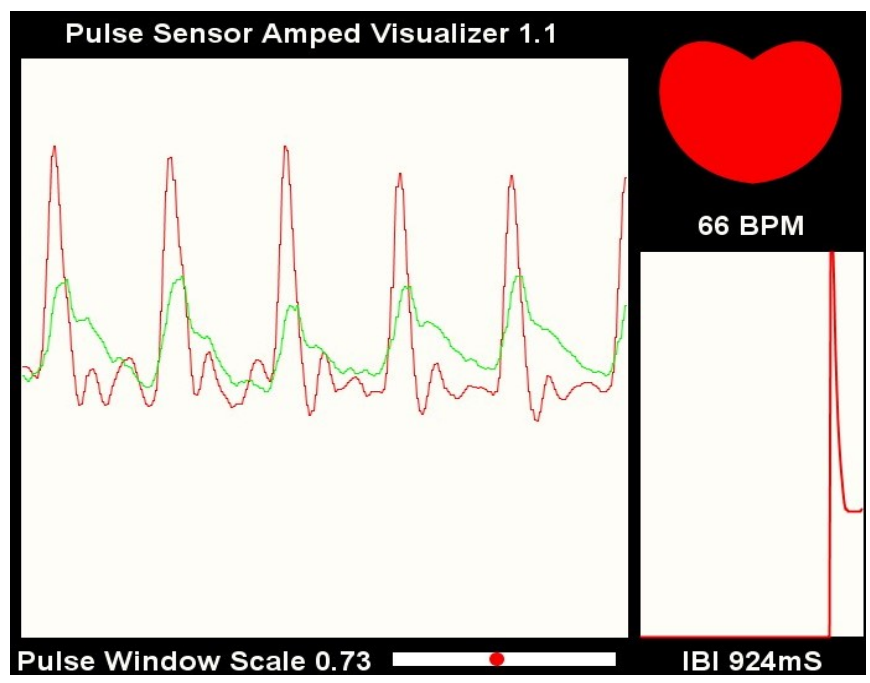

Figure 7.7: Pulse signals detected from the Arduino board and the PulseSensor 


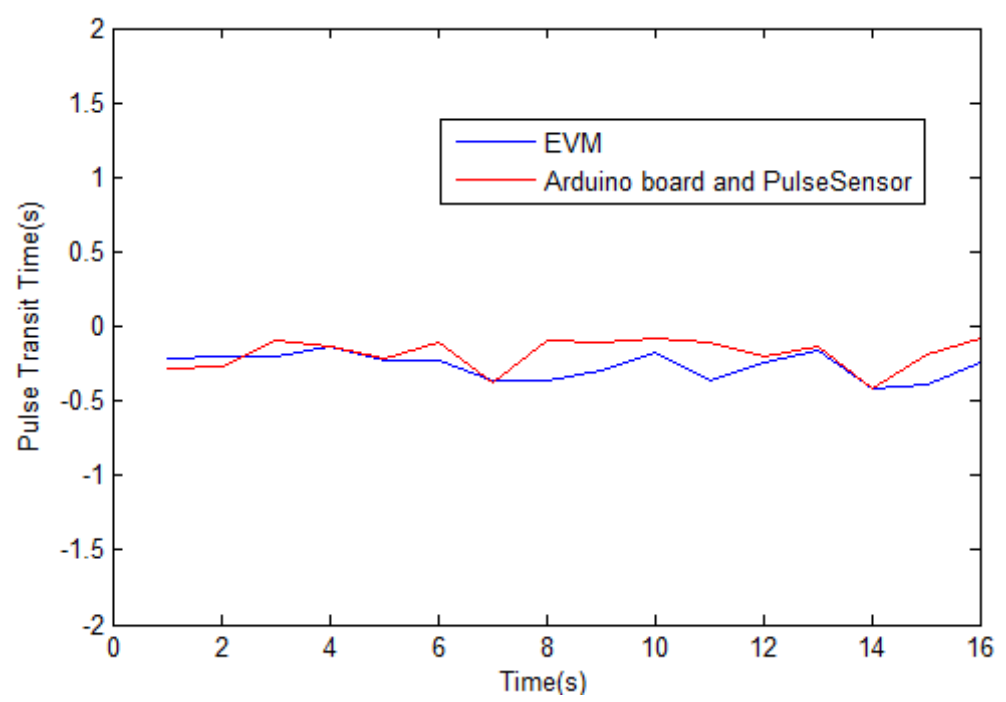

Figure 7.8: Comparison of the time difference between the detected pulses on the wrist and the neck through the usage of EVM and the Arduino board with PulseSensor

Figure 7.8 illustrates the calculated PTT. The blue curve and the red curve represent the time difference between the detected pulses on the wrist and neck through the usage of EVM and the Arduino board and PulseSensor, respectively. We can see the pulse signals and the time differences between the wrist and the neck obtained by the two methods are similar. Therefore, the Eulerian color magnification method can satisfy the measurement requirement in a more convenient and non-intrusive way.

\subsection{Summary and Conclusion}

Smart phone and high resolution cameras have been involved in our daily health-care monitoring. The main contribution of this study was to propose a non-intrusive way to observe the blood flow and to obtain the PTT. A novel method called Eulerian color magnification framework was introduced to measure the PTT. By doing so, the PTT can 
be calculated without any former knowledge of the ECG and PPG signals. This can be simply calculated from the video recorded by the camera.

In order to evaluate our proposed system, the comparison of the PTT between the proposed system and the real pulse sensor collection was studied. In this way, the Eulerian color magnification framework can be employed to measure some biomedical signals in a non-intrusive way, and the EVM framework can be used to process the information from the camera for health-care monitoring. 


\section{Chapter 8}

\section{Conclusion and Future Work}

\subsection{Conclusion}

In conclusion, this thesis has investigated several approaches in measuring the pulse transit time (PTT) and estimating the arterial blood pressure in a continuous and noninvasive way. Two approaches were proposed for signal enhancement to improve the accuracy of PTT measurement. The first one was through the enhancement of the ECG and PPG signals. The other one was through the application of the Eulerian video magnification method.

The main contributions of this thesis are summarized as follows:

$>$ A new ECG enhancement method was proposed based on EEMD and the adaptive filtering technique to eliminate the contact noise contained in the ECG signals obtained from wearable medical monitoring sensors [27][28].

$>$ The EMD-based algorithm was introduced into the acoustic echo cancellation field and a new EMD-based sub-band adaptive filtering structure was designed. The de-noise ability of the EMD-based algorithm was demonstrated [29]. 
$>$ The correlation between blood pressure and pulse transit time was evaluated and a cuff-less non-intrusive approach was investigated to estimate arterial blood pressure continuously [30].

$>$ Three secondary peak detection methods were proposed to reveal the secondary peak from the original PPG signal. The correlation between the arterial blood pressure and the pulse transit time was improved, and the dicrotic notch impact on the PPG signal was removed [31].

$>$ The wrist pulse signal was noninvasively measured by the Eulerian video magnification (EVM) framework. The two-Gaussian curve modeling method was used to analyze the detected wrist pulse signal [32].

A video-based non-intrusive method was proposed to display the blood flow and to measure the PTT. The EVM framework was introduced and applied to calculate the time difference between different spots on the human skin surface [33].

The above results obtained in this thesis demonstrate that the enhanced ECG and PPG signals can improve the accuracy of the PTT in estimating the arterial blood pressure. The EVM method can capture the characteristics of the wrist pulse signal and detect the heart pulse on different areas of the human skin. This paves the way towards the usage of cameras for continuous arterial blood pressure monitoring.

\subsection{Future Work}

The work presented in this thesis focuses on measuring the PTT more accurately. The thesis investigated the usage of the ECG and PPG signals to estimate the PTT and correlate to the 
arterial blood pressure. It was also shown that the PPG signal alone has the potential to estimate the arterial blood pressure. More characteristic parameters of the PPG signal can be extracted to detect the primary peak. Future work into the correlation between these parameters and the arterial blood pressure could lead to a better non-intrusive method to measure the blood pressure.

The PTT-based approach to estimate the arterial blood pressure variations requires a calibration procedure to form individual-dependent mapping of the obtained PTT to the blood pressure values. Future work can explore the automation of this calibration procedure to design of a continuous blood pressure monitoring system. The periodicity of recalibration, the number of trials for calibration, and the accuracy of the calibration could be investigated. Furthermore, the usage of EVM can be explored to capture the wrist pulse signal, analyze it and classify various diseases using the appropriate pattern recognition and classification techniques. Also, as discussed in [201], the combination of EVM and other signal enhancement methods has a high potential to extract useful information on the health status of the patients.

More clinical studies are needed with a large number of subjects to demonstrate the ability of the PTT measured by the EVM method in estimating the arterial blood pressure. Experiment has been taken to compare the estimated blood pressure with the blood pressure measured by the Omron Blood Pressure Monitor Series 10 [202], which can calibrate for each patient automatically and display the systolic blood pressure, diastolic blood pressure, and the pulse rate after each measurement. The preliminary results are very promising. 


\section{Appendices}

\section{Appendix A: EMD-based Sub-band Adaptive Filtering for Acoustic Echo Cancellation}

Acoustic echo cancellation (AEC) is one of the most severe requirements in hands-free telephone and teleconference communication [155]. In previous research, the reverberant speech originating from different rooms was trained to combat the effect of testing with reverberant speech [156]. In order to further demonstrate the de-noise ability of the EMDbased algorithm, we propose an EMD-based sub-band adaptive filtering structure, which applies the EMD-based algorithm dealing with the far-end speech signal and the microphone output to obtain two sets of intrinsic mode functions (IMFs). In addition, each IMF set is separated into different bands based on the power spectral density (PSD) of every IMF. Experiment signals were collected from a medium-size office room and simulations were taken under different conditions by three types of EMD-based algorithms. Results show that the proposed structure is able to model the transfer function of the unknown environment, and the ensemble EMD (EEMD) algorithm and the noisemodulated EMD (NEMD) have better performance than the EMD algorithm in terms of echo return loss enhancement. 
Compared with the full-band adaptive filtering technique, several studies [157][158] have proved that the sub-band adaptive filtering technique has better performance, especially when the impulse response is long [159][160]. The basic structure of the normalized sub-band adaptive filter (NSAF) system [161] is shown in Figure 9.1.

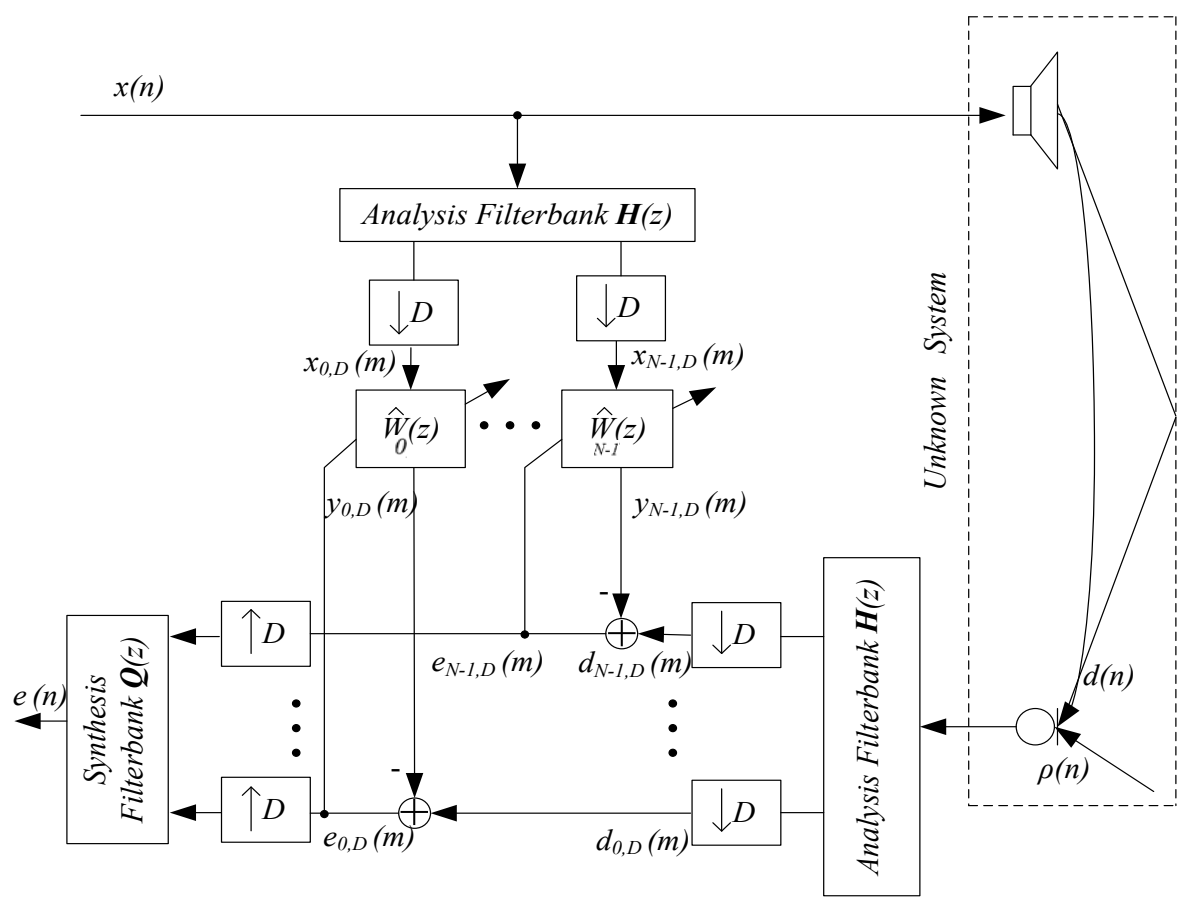

Figure 9.1: Structure of NSAF system

The structure of the proposed EMD-based sub-band adaptive filtering system is shown in Figure 9.2, where $x(n), d(n)$ are the far end signal and the microphone output, respectively. $e(n)$ is the output error. In [162] it was pointed out that for a loss-less filter bank, the summation of the powers of each sub-band error was equal to the power of the output error. In addition, due to the simplicity of the normalized least mean squares (NLMS), it is used as the adaptive filtering algorithm in the sub-bands. 


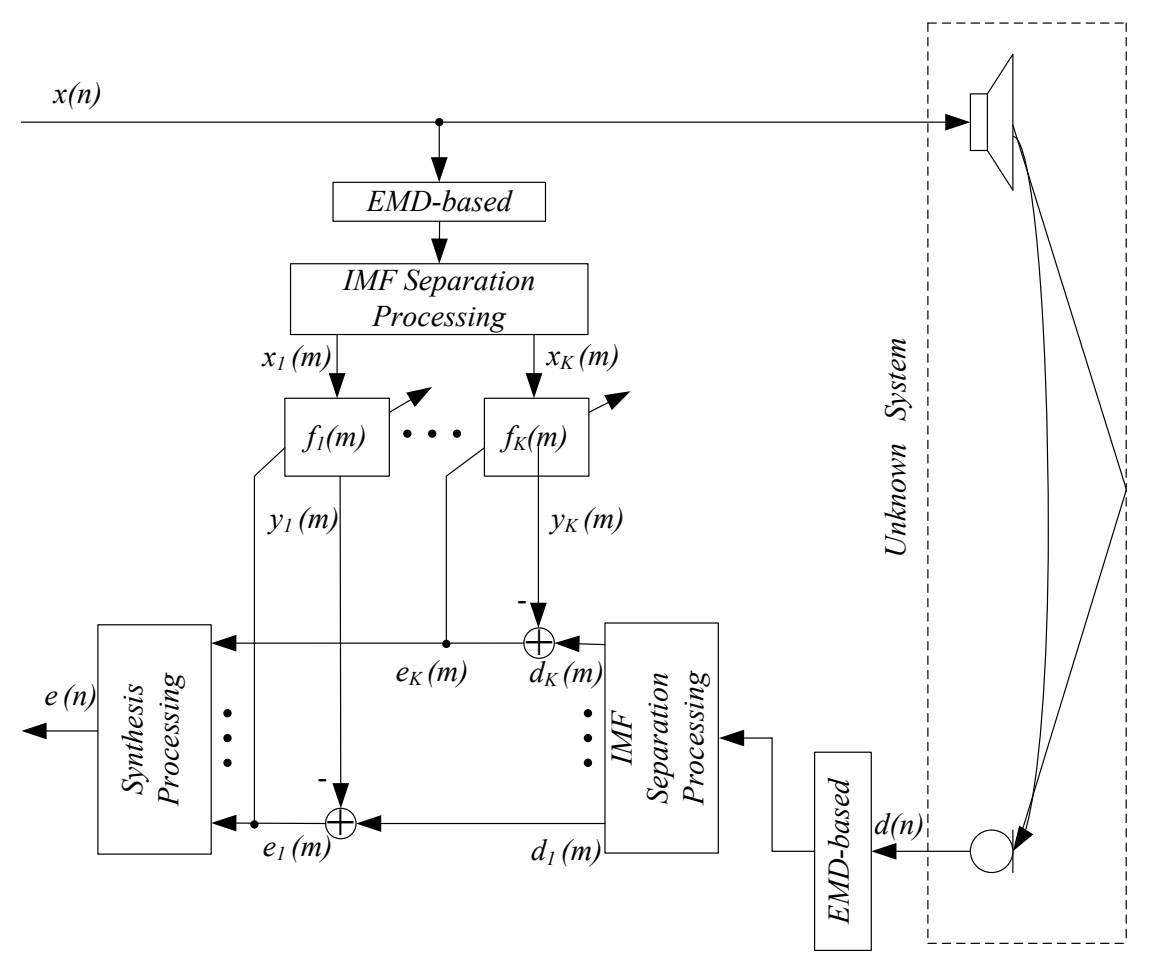

Figure 9.2: Structure of the EMD-based sub-band adaptive filtering system

From Figure 9.3, we can see that when applying the EMD-based algorithm to echo cancellation, the echo return loss enhancement (ERLE) value is increasing as the sub-band number changing from two to eight, which is consistent with the previous work on the acoustic echo cancellation [163][164]. This, in one aspect, proves the proposed system is suitable to model the echo environment. 


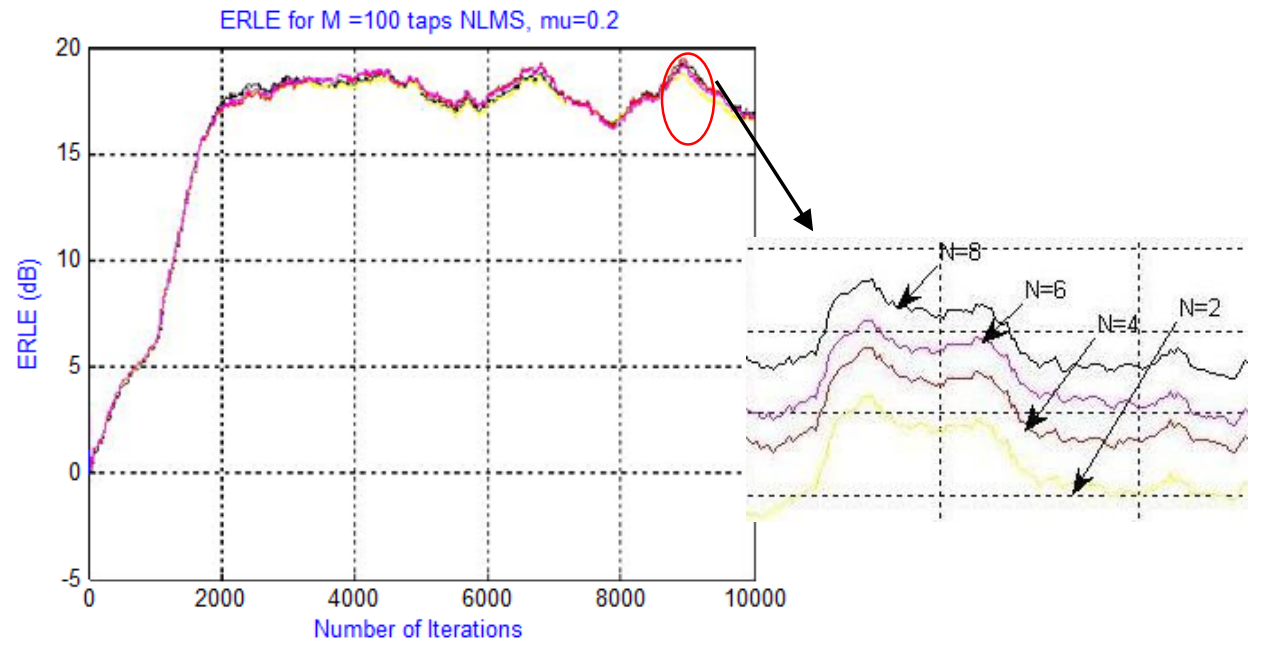

Figure 9.3: ERLE values of the EEMD sub-band adaptive filtering structure with subband number $\mathrm{N}=2,4,6,8$

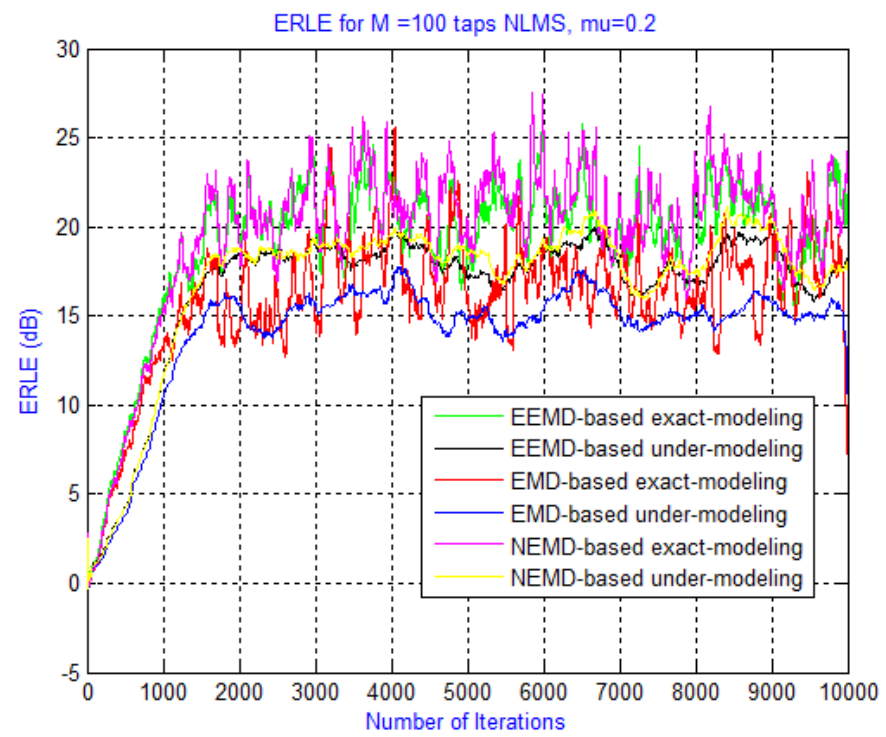

Figure 9.4: ERLE values of different EMD-based sub-band adaptive filtering system under the exact-modeling and under-modeling scenarios

There are two scenarios considered in this study. One is the exact-modeling scenario, when the length of the impulse response and the length of the adaptive filter are equal. The 
other one is the under-modeling scenario, when the length of the impulse response is greater than the length of the adaptive filter. Figure 9.4 illustrates the ERLE of different EMD-based algorithm under these two scenarios.

As shown in Figure 9.4, the performance of the EEMD and NEMD sub-band adaptive filtering system is better than that of the EMD, and all the three systems have better convergence under the exact-modeling scenario. The results also demonstrate that the EEMD and NEMD algorithms are able to conquer the mode-mixing problems existing in the EMD algorithm through different aspects. 


\section{List of References}

[1] C. J. Murray, J. A. Lauer, R. C. Hutubessy, L. Niessen, N. Tomijima, A. Rodgers, C. M. Lawes, D. B. Evans, "Effectiveness and costs of interventions to lower systolic blood pressure and cholesterol: a global and regional analysis on reduction of cardiovascular-disease risk," The Lancet, vol. 361, pp. 717-725, March 2003.

[2] J. J. Rutherford, "Wearable technology," IEEE Engineering in Medicine and Biology Magazine, vol. 29, pp. 19-24, May-June 2010.

[3] S. Ahmad, M. Bolic, H. Dajani, V. Groza, I. Batkin, S. Rajan, "Measurement of heart rate variability using an oscillometric blood pressure monitor," IEEE Transactions on Instrumentation and Measurement, vol. 59, pp. 2575-2590, October 2010.

[4] P. Dupuis and C. Eugene, "Combined detection of respiratory and cardiac rhythm disorders by high-resolution differential cuff pressure measurement," IEEE Transactions on Instrumentation and Measurement, vol. 49, pp. 498-502, June 2000.

[5] A. Silvani, D. Grimaldi, S. Vandi, G. Barletta, R. Vetrugno, F. Provini, G. Pierangeli, C. Berteotti, P. Montagna, G. Zoccoli, P. Cortelli, " Sleep-dependent changes in the coupling between heart period and blood pressure in human subjects," American Journal of Physiology - Regulatory, Integrative and Comparative Physiology, vol. 294, pp. 1686-1692, May 2008.

[6] S. Ahmad, S. Chen, K. Souerdan, I. Batkin, M. Bolic, H. Dajani, V. Groza, "A prototype of an integrated blood pressure and electrocardiogram device for multiparameter physiologic monitoring," in Proc. of the IEEE Conference on Instrumentation and Measurement Technology, Austin, TX, May 2010, pp. 12441249.

[7] G. Parati, J. E. Ochoa, C. Lombardi, G. Bilo, "Assessment and management of blood pressure variability," Nat. Rev. Cardiol., vol. 10, no. 3, pp. 143-155, March 2013.

[8] J. A. Kitterman, R. H. Phibbs, W. H. Tooley, "Catheterization of umbilical vessels in newborn infants," Pediatric Clinics of North America, vol. 17, pp. 895-912, November 1970.

[9] S. Saponara, M. Donati, T. Bacchillone, L. Fanucci, I. Sanchez-Tato, C. Carmona, P. Barba, "Remote monitoring of vital signs in parients with chronic heart failure: 
sensor devices and data analysis perspective," in Proc. of the IEEE Conference on Sensors Applications Symposium (SAS), Brescia, February 2012, pp. 1-6.

[10] E.Balestrieri and S. Rapuano, "Instruments and methods for calibration of oscillometric blood pressure measurement devices," IEEE Transactions on Instrumentation and Measurement, vol. 59, pp. 2391-2404, September 2010.

[11] T. Weber, S. Wassertheurer, M. Rammer, E. Maurer, B. Hametner, C. C. Mayer, J. Kropf, B. Eber, "Validation of a brachial cuff-based method for estimating central systolic blood pressure," Hypertension, vol. 58, pp. 825-832, November 2011.

[12] M. V. Moer, K. Barbe, "Influence of the cuff deflation mode on oscillometric blood pressure measurements," in Proc. of the IEEE International Symposium on Medical Measurements and Applications (MeMeA), Bari, May 2011, pp. 652-656.

[13] E. A. Zorn, M. B. Wilson, J. J. Angel, J. Zanella, B. S. Alpert, "Validation of an automated arterial tonometry monitor using association for the advancement of medical instrumentation standards," Blood Pressure Monitoring, vol. 2, pp. 185-188, August 1997.

[14] K. Jagomagi, J. Talts, R. Raamat, E. Lansimies, "Technical note: continuous noninvasive measurement of mean blood pressure in fingers by volume-clamp and differential oscillometric method," Clinical Physiology, vol. 16, pp. 551-560, September 1996.

[15] Y. Miyauchi, S. Koyama, H. Ishizawa, "Basic experiment of blood-pressure measurement which uses FBG sensors," in Proc. of the IEEE International Conference on Instrumentation and Measurement, Minneapolis, MN, May 2013, pp. 1767-1770.

[16] L. A. Geddes, M. H. Voelz, C. F. Babbs, J. D. Bourland, W. A. Tacker, "Pulse transit time as an indicator of arterial blood pressure," Psychophysiology, vol. 18, pp. 71-74, January 1981.

[17] E. Pinheiro, O. Postolache, P. Girao, "Pulse arrival time and ballistocardiogram application to blood pressure variability estimation," in Proc. of the IEEE International Workshop on Medical Measurements and Applications (MeMeA), Cetraro, May 2009, pp. 132-136.

[18] T. Kanda, E. Nakamura, T. Moritani, Y. Yamori, "Arterial pulse wave velocity and risk factors for peripheral vascular diseas," Eur. J. Appl. Physiol, vol. 82, pp.1-7, 2000 .

[19] R. P. Smith, J. Argod, J. L. Pepin, P. A. Levy, "Pulse transit time: an appraisal of potential clinical applications," Thorax, vol. 54, pp. 452-845, 1999. 
[20] M. H. Pollak, P. A. Obrist, "Aortic-radial pulse transit time and ECG Q-wave to radial pulse wave intervals as indices of beat-by-beat blood pressure change," Psychophysiology, vol. 20, pp. 21-28, 1983.

[21] R. Shriram, A. Wakankar, N. Daimiwal, D. Ramdasi, "Continuous cuffless blood pressure monitoring based on PTT," in Proc. of the International Conference on Bioinformatics and Biomedical Technology (ICBBT), Chengdu, April 2010, pp. 5155 .

[22] G. Fortino and V. Giampa, "PPG-based methods for non-invasive and continuous blood pressure measurement: an overview and development issues in body sensor networks," in Proc. of the IEEE International Workshop on Medical Measurements and Applications (MeMeA), Ottawa, ON, April 2010, pp. 10-13.

[23] M. Y. M. Wong, C. C. Y. Poon, Y. T. Zhang, "An evaluation of the cuffless blood pressure estimation based on pulse transit time technique: a half year study on normotensive subjects," Cardiovasc Eng., vol. 9, no. 1, pp. 32-38, March 2009.

[24] J. Muehlsteff, X. L. Aubert, M. Schuett, "Cuffless estimation of systolic blood pressure for short effort bicycle tests: the prominent role of the pre-ejection period," in Proc. of 28th Annual International Conference of the IEEE Engineering in Medicine and Biology Society (EMBC), New York, NY, August 2006, pp. 50885092.

[25] S. Deb, C. Nanda, D. Goswami, J. Mukhopadhyay, S. Chakrabarti, "Cuff-less estimation of blood pressure using pulse transit time and pre-ejection period," in Proc. of the International Conference on Convergence Information Technology, Gyeongju, November 2007, pp. 941-944.

[26] W. B. Gu, C. C. Y. Poon, Y. T. Zhang, "A novel parameter from PPG dicrotic notch for estimation of systolic blood pressure using pulse transit time," in Proc. of the International Summer School and Symposium on Medical Devices and Biosensors (ISSS-MDBS), Hong Kong, June 2008, pp. 86-88.

[27] X. He, R. A. Goubran, X. P. Liu, "Signal enhancement of wearable ECG monitoring sensors based on ensemble empirical mode decomposition," in Proc. of the IEEE International Symposium on Medical Measurements and Applications (MeMeA), Bari, May 2011, pp. 433-436.

[28] X. He, R. A. Goubran, X. P. Liu, "Ensemble empirical mode decomposition and adaptive filtering for ECG signal enhancement," in Proc. of the IEEE International Symposium on Medical Measurements and Applications (MeMeA), Budapest, May 2012, pp. 136-140.

[29] X. He, R. A. Goubran, X. P. Liu, "EMD-based sub-band adaptive filtering for acoustic echo cancellation," International Journal of Speech Technology, vol. 17, pp. 37-42, March 2014. 
[30] X. He, R. A. Goubran, X. P. Liu, "Evaluation of the correlation between blood pressure and pulse transit time," in Proc. of the IEEE International Symposium on Medical Measurements and Applications (MeMeA), Gatineau, QC, May 2013, pp. 17-20.

[31] X. He, R. A. Goubran, X. P. Liu, "Secondary peak detection of PPG signal for continuous cuff-less arterial blood pressure measurement," IEEE Transactions on Instrumentation and Measurement, vol. 63, no. 6, pp. 1431-1439, June 2014.

[32] X. He, R. A. Goubran, X. P. Liu, "Wrist pulse measurement and analysis using Eulerian video magnification," in Proc. of the IEEE International Conference on Biomedical and Health Informatics, Las Vegas, February 2016.

[33] X. He, R. A. Goubran, X. P. Liu, "Using Eulerian Video Magnification Framework to Measure Pulse Transit Time," in Proc. of the IEEE International Symposium on Medical Measurements and Applications (MeMeA), Lisbon, June 2014, pp. 50-53.

[34] J. R. Levick, "An introduction to cardiovascular physiology, fourth edition," Arnold Publishers, New York, 2003.

[35] "Abstract illustration of circulatory system from Blausen," Available: https://en. wikipedia.org/wiki/Talk: Circulatory_system.

[36] A. C. Guyton and J. E. Hall, "Textbook of Medical Physiology, 11 th edition" Elsevier Saunder, Philadelphia, 2006

[37] Q. Destiny and S. B. Xavax, "Wiggers Diagram," Wikimedia Commons, March, 2012. Available: https://en.wikipedia.org/wiki/Cardiac_cycle\#/media/File:Wiggers_ Diagram.png.

[38] R. E. Klabunde, "Cardiovascular Physiology Concepts, second edition," Lippincott Williams \& Wilkins, Philadelphia, 2011.

[39] P. M. Kearney, M. Whelton, K. Reynolds, P. Muntner, P. K. whelton, H. He, "Global burden of hypertension: analysis of worldwide date," Lancet., vol. 365, no. 9455, pp. 217-223, January 2005.

[40] P. K. Whelton, J. He, P. Muntner, "Prevalence, awareness, treatment, and control of hypertension in North America, North Africa, and Asia," J. Hum. Hypertens., vol. 18, no. 8, pp. 545-551, August 2004.

[41] "Understanding blood pressure readings," American Heart Association, January 2011.

[42] Mayo Clinic staff, "Low blood pressure (hypotension) — causes," Mayo Foundation for Medical Education and Research, May 2009. 
[43] H. M. Berge-Landry, D. H. Bovbjer, G. D. James, "Relationship between wakingsleep blood pressure and catecholamine changes in African-American and EuropeanAmerican women". Blood Press Monit., vol. 13, no. 5, pp. 257-262, October 2008.

[44] T. W. Hansen, Y. Li, J. Boggia, L. Thijs, T. Richart, J. A. Staessen, "Predictive Role of the Nighttime Blood Pressure," Hypertension, vol. 57, no. 1, pp. 3-10, November 2011.

[45] D. S. Baim, "Grossman's cardiac catheterization, angiography, and intervention, seventh edition" Lippincott Williams \& Wilkins, Philadelphia, 2006.

[46] A. D. Vecchi, R. E. Clough, N. R. Gaddum, M. C. Rutten, P. Lamata, T. Schaeffter, D. A. Nordsletten, N. P. Smith, "Catheter-induced errors in pressure measurements in vessels: an in-vitro and numerical study," IEEE Transactions on Biomedical Engineering, vol. 61, no. 6, pp. 1844-1850, June 2014.

[47] L.A. Geddes, "Handbook of blood pressure measurement," Humana Press, 1991.

[48] J. Herisson, "The sphygmomanomert, an instrument which renders the action of arteries apparent to the eye with improvement of the instrument and prefatory remarks by the translator," Longman, London, 1835.

[49] M. Gavaghan, "Vascular hemodynamics," AORN Journal, January 1998.

[50] E. O'Brien, "A short history of blood pressure measurement," Available: http://www. bloodpressurehistory.com/history.html, 2011.

[51] Y. L. Shevchenko and J. E. Tsitlik, " $90^{\text {th }}$ anniversary of the development by Nikolai S. Korotkoff of the auscultatory method of measuring blood pressure," Circulation, vol. 94, no. 2, pp. 116-118, July 1996.

[52] "Blood pressure measurement using the auscultatory method based on the (first) korotkoff-sound," Available: https://en.wikipedia.org/wiki/Korotkoff_sounds\#/ media/File:Korotkow_deutsch.png.

[53] T. G. Pickering, J. E. Hall, L, J. Appel, B. E. Falkner, J. Graves, M. N. Hill, "Recommendations for blood pressure measurement in humans and experimental animals: Part 1: blood pressure measurement in humans: a statement for professionals from the Subcommittee of Professional and Public Education of the American Heart Association Council on High Blood Pressure Research," Hypertension, vol. 45, pp. 142-161, January 2005.

[54] M. Ramsey $3^{\text {rd }}$, "Blood pressure monitoring: automated oscillometric devices," $J$. Clin. Monit., vol. 7, no. 1, pp. 56-57, January 1991.

[55] J. A. Staessen, R. Asmar, M. D. Buyzere, Y. Imai, G. Parati, K. Shimada, G. Stergiou, J. Redon, P. Verdecchia, "Task force II: blood pressure measurement and 
cardiovascular outcome," Blood Press Monit., vol. 6, no. 6, pp. 355-370, December 2001.

[56] G. M. Drzewiecki, J. Melbin, A. Noordergraaf, "Arterial tonometry: review and analysis," J. Biomech., vol. 16, no. 6, pp. 141-152, 1983.

[57] Y. F. Cheung, "Arterial stiffness in the young: assessment, determinations, and implications," Korean Circulation Journal, vol. 40, no. 4, pp.153-162, April 2010.

[58] K. Matthys and P. Verdonck, "Development and modelling of arterial applanation tonometry: a review," Technol. Health Care, vol. 10, no. 1, pp. 65-76, 2002.

[59] K. Belani, M. Ozaki, J. Hynson, T. Hartmann, H. Reyford, J. M. Martino, M. Poliac, R. Miller, "A new noninvasive method to measure blood pressure: result of a multicenter trial," Anesthesiology, vol. 91, no. 3, pp. 686-692, September 1999.

[60] S. Skinner, "A clinical guide to pulse wave analysis," A. Medical, Ed., 2009.

[61] J. Penaz, "Photoelectric measurement of blood pressure, volume and flow in the finger," in Proc. of the International Conference in Medicine and Biological Engineering, Dresden, 1973, pp. 104-144.

[62] G. Mancia, R. Fagard, K. Narkiewicz, J. Redón, A. Zanchetti, M. Böhm, T. Christiaens, R. Cifkova, G. D. Backer, A. Dominiczak, M. Galderisi, D. E. Grobbee, T. Jaarsma, P. Kirchhof, S. E. Kjeldsen, S. Laurent, A. J. Manolis, P. M. Nilsson, L. M. Ruilope, R. E. Schmieder, P. A. Sirnes, P. Sleight, M. Viigimaa, B. Waeber, F. Zannad, Task Force Members, "2013 ESH/ESC Guidelines for the management of arterial hypertension: The Task Force for the management of arterial hypertension of the European Society of Hypertension (ESH) and of the European Society of Cardiology (ESC)," J. Hypertens., vol. 31, no. 7, pp. 1281-1357, July 2013.

[63] Asmar, "Arterial stiffness and pulse wave velocity," Elsevier, Paris, 1999.

[64] M. Butlin, "Structural and function effects on large artery stiffness: an in-vivo experimental investigation," PhD thesis, University of New South Wales, Australia, October 2007.

[65] W. B. White, A. S. Berson, C. Robbins, M. J. Jamieson, L. M. Prisant, E. Roccella, S. G. Sheps, "National standard for measurement of resting and ambulatory blood pressures with automated sphygmomanometers," Hypertension, vol. 21, pp. 504-509, 1993.

[66] E. O'Brien, J. Petrie, W. A. Littler, P. L. Padfield, K. O'Malley, M. Jamieson, “The British Hypertension Society protocol for the evaluation of automated and semiautomated blood pressure measuring devices with special reference to ambulatory systems," J. Hypertens., vol. 8, no. 7, pp. 607-619, July 1990. 
[67] M. Faber and G. Miolller-Hou, "The human aorta. V. Collagen and elastin in the normal and hypertensive aorta," Acta. Pathol. Microbiol. Scand., vol. 31, no. 3, pp. 377-382, 1952.

[68] G. M. London, A. P. Guerin, B. Pannier, S. J. Marchais, M. Stimpel, "Influence of sex on arterial hemodynamics and blood pressure. Role of body height," Hypertension, vol. 26, no. 3, pp. 514-519, September 1995.

[69] S. M. Hughes, L. J. Dixon, G. E. McVeigh "Arterial stiffness and pulse wave velocity: problems with terminology," Circulation, vol. 109, no. 2, pp. e2-e3, January 2004.

[70] A. Benetos, C. Adamopoulos, J. M. Bereau, M. Temmar, C. Labat, K. Bean, F. Thomas, B. Pannier, R. Asmar, M. Zureik, M. Safar, L. Guiza, "Determinants of accelerated progression of arterial stiffness in normotensive subjects and in treated hypertensive subjects over a 6-year period," Circulation, vol. 105, no. 10, pp. 12021207, March 2002.

[71] D. B. Wax, H. M. Lin, A. B. Leibowitz, "Invasive and concomitant noninvasive intraoperative blood pressure monitoring: observed differences in measurements and associated therapeutic interventions," Anesthesiology, vol. 115, no. 5, pp. 973-978, November 2011.

[72] M. E. Safar, O. Henry, S. Meaume, "Aortic pulse wave velocity: an independent marker of cardiovascular risk," The American Journal of Geriatric Cardiology, vol. 11, no. 5, pp. 295-298, October 2002.

[73] D. J. Pitson, A. Sandell, R. V. Hout, J. R. Stradling "Use of pulse transit time as a measure of inspiratory effort in patients with obstructive sleep apnoesa," Eur. Perpir. Jour., vol. 8, no. 10, pp. 1669-1674, October 1995.

[74] S. N. Kounalakis and N. D. Geladas, "The role of pulse transit time as an index of arterial stiffness during exercise," Cardiovasc. Eng., vol. 92, no. 3, pp. 92-97, September 2009.

[75] S. Mazaheri and E. Zahedi, "A comparative review of blood pressure measurement methods using pulse wave velocity," in Proc. of IEEE International Conference on Smart Instrumentation, Measurement and Applications (ICSIMA), Kuala Lumpur, November 2014, pp. 1-5.

[76] L. A. Geddes, M. H. Voelz, C. F. Babbs, J. D. Bourland, W. A. Tacker, "Pulse transit time as an indicator of arterial blood pressure," Psychophysiology, vol. 18, no. 1, pp. 71-74, January 1981.

[77] G. V. Marie, C. P. Lo, J. V. Jones, D. W. Johnston, "The relationship between arterial blood pressure and pulse transit time during dynamic and static exercise," Psychophysiology, vol. 21, no. 5, pp. 521-527, September 1984. 
[78] P. Fung, G. Dumont, C. Ries, C. Mott, M. Ansermino, "Continuous noninvasive blood pressure measurement by pulse transit time," in Proc. of IEEE International Conference in Medicine and Biology, San Francisco, CA, September 2004, pp. 738741.

[79] T. Ma and Y. T. Zhang, "A correlation study on the variabilities in pulse transit time, blood pressure, and heart rate recorded simultaneously from healthy subjects," in Proc. of IEEE Annual Conference in Medicine and Biology, Shanghai, September 2005, pp. 996-999.

[80] C. C. Y. Poon and Y. T. Zhang, "Cuff-less and noninvasive measurement of arterial blood pressure by pulse transit time," in Proc. of IEEE Annual Conference in Medicine and Biology, Shanghai, September 2005, pp. 5877-5880.

[81] C. H. Chan and Y. T. Zhang, "Continuous and long-term arterial blood pressure monitoring by using h-shirt," in Proc. of International Conference on Information Technology and Application in Biomedicine, Shenzhen, May 2008, pp. 267-269.

[82] P. S. Pandian, K. Mohanavelu, K. P. Safeer, T. M. Kotresh, D. T. Shakunthala, P. Gopal, V. C. Padaki, "Smart vest: wearable multi-parameter remote physiological monitoring system," Medical Engineering and Physics, vol. 30, no. 4, pp. 466-477, May 2008.

[83] V. Chandrasekaran, R. Dantu, S. Jonnada, S. Thiysgaraja, K. P. Subbu, "Cuffless differential blood pressure estimation using smart phones," IEEE Transactions on Biomedical Engineering, vol. 60, no. 4, pp. 1080-1089, April 2013.

[84] R. Asmar, A. Benetos, J. Topouchian, P. Laurent, B. Pannier, A. M. Brisac, R. Target, B. I. Levy, "Assessment of arterial distensibility by automatic pulse wave velocity measurement. Validation and clinical application studies," Hypertension, vol. 26, no. 3, pp. 485-490, September 1995.

[85] M. W. Rajzer, W. Wojciechowska, M. Klocek, I. Palka, M. Brzozowska-Kiszka, K. Kawecka-Jaszcz, "Comparison of aortic pulse wave velocity measured by three techniques: Complior, SphygmoCor and Arteriograph," J. Hypertens., vol. 26, no. 10, pp. 2001-2007, October 2008.

[86] R. D. Latham, N. Westerhof, P. Sipkema, B. J. Rubal, P. Reuderink, J. P. Murgo, "Regional wave travel and reflections along the human aorta: a study with six simultaneous micromanometric pressures," Circulation, vol. 72, no. 6, pp.12571269, December 1985.

[87] W. W. Nichols and M. F. O'Rourke, "Aortic pulse wave velocity, reflection site distance, and augmentation index," Hypertension, vol. 53, vol. 1, pp. e9, January 2009.

[88] R. P. Smith, J. Argod, J. Pepin, P. A. Levy, "Pulse transit time: an appraisal of potential clinical applications," Thorax, vol. 54, no. 5, pp. 452-457, May 1999. 
[89] J. G. Webster, "The measurement, instrumentation, and sensors handbook," $C R C$ Press, Boca Raton, Fla, 1999.

[90] D. E. Mohrman and L. J. Heller, "Cardiovascular physiology, $8^{\text {th }}$ edition," Lange, USA, 2011.

[91] "The cardiac cycle as correlated to the ECG," Available: https://en.wikipedia.org/ wiki/Heart.

[92] N. V. Thakor, "Electrocardiographic monitors," Encyclopedia of Medical Devices and Instrumentation, John Wiley \& Sons, New York, pp. 1002-1017, 1988.

[93] C. E. Kossmann, D. A. Brody, G. E. Burch, O. H. Schmitt, "Recommendations for standardization of leads and specifications for instruments in electrocardiography and vectorcardiography," Circulation, vol. 35, no. 3, pp. 583-602, February 1967.

[94] “ECG electrodes,” Available: https://commons.wikimedia.org/wiki/File:Limb_leads 2_ENG.svg.

[95] "Placement of the precordial electrodes" and "The limb leads and augmented limb leads," Available: https://en.wikipedia.org/wiki/Electrocardiography.

[96] A. Gruetzmann, S. Hansen, J. Muller, "Novel dry electrodes for ECG monitoring," Physiol. Meas., vol. 28, no. 11, pp. 1375-1390, November 2007.

[97] P. J. Xu, H. Zhang, X. M. Tao, "Textile-structured electrodes for electrocardiogram," Textile Progress, vol. 40, no. 4, pp. 183-213, December 2008.

[98] E. B. Joffe and K-S. Lock, "Grounds for grounding: a circuit to system handbook," Wiley-IEEE Press, January 2010.

[99] M. F. Chimene and R. Pallas-Areny, "A comprehensive model for power line interference in biopotential measurements," IEEE Transactions on Instrumentation and Measurement, vol. 49, no. 3, pp. 535-540, June 2000.

[100]O. Sayadi and M. B. Shamsollahi, "Model-based fiducial points extraction for baseline wandered electrocardiograms," IEEE Transactions on Biomedical Engineering, vol. 55, no. 1, pp. 347-351, January 2008.

[101]G. Friesen, T. C. Jannett, M. A. Jadallah, S. L. Yates, S. R. Quint, H. T. Nagle, "A comparison of the noise sensitivity of nine QRS detection algorithms," IEEE Transactions on Biomedical Engeering, vol. 37, no. 1, pp. 85-98, January 1990.

[102]A. L. Goldberger, L. A. N. Amaral, L. Glass, J. M. Hausdorff, P. C. Ivanov, R. G. Mark, J. E. Mietus, G. B. Moody, C. -K. Peng, H. E. Stanley, "PhysioBank, PhysioToolkit, and PhysioNet: components of a new research resource for complex physiologic signals," Circulation, vol. 101, no. 23, pp. 215-220, September 2000. 
[103]“MIT-BIH Arrhythmia Database," Available: http://www.physionet.org/physiobank/ database $/ \mathrm{mitdb} /$.

[104]"MIT-BIH noise stress test database," Available: http://www.physionet.org/ physiobank /database/nstdb/.

[105]Blanco-Velasco, M., Weng, B., Barner, K.E., "ECG signal denoising and baseline wander correction based on the empirical mode decomposition," Comput. Biol. Med., vol. 38, no. 1, pp. 1-13, January 2008.

[106]J. M. Leski, N. Henzel, "ECG baseline wander and powerline interference reduction using nonlinear filter bank," Signal Processing, vol. 35, no. 4, pp. 781-793, April 2004.

[107]N. V. Thakor and Y. Zhu, "Application of adaptive filtering to ECG analysis: noise cancellation and arrhythmia detection," IEEE Transaction on Biomedical Engineering, vol. 38, no. 8, pp. 785-794, August 1991.

[108]P. E. Tikkanen, "Nonlinear wavelet and wavelet packet denoising of electrocardiogram signal," Biol. Cybern., vol. 80, no. 4, pp. 259-267, April 1999.

[109]A. K. Barros, A. Mansour, N. Ohnishi, "Removing artifacts from electrocardiographic signals using independent components analysis," Neurocomputing, vol. 22, no. 1-3, pp. 173-186, November 1998.

[110]F. N. Ucar, M. Korurek, E. Yazgan, "A noise reduction algorithm in ECG signals using wavelet transform," in Proc. of $2^{\text {nd }}$ International Conference on Biomedical Engineering Days, Istanbul, May 1998, pp. 36-38.

[111]Y. Liu and M. G. Pecht, "Reduction of skin stretch induced motion artifactss in electrocardiogram monitoring using adaptive filtering," in Proc. of the IEEE EMBS 28th Annual International Conference, New York, NY, August 2006, pp. 6045-6048.

[112]B. Widrow, “Adaptive signal processing,” Prentice-Hall Inc., New Jersey, 1985.

[113]B. Widrow, J. R. Glover, J.M. McCool, J. Kaunitz, “Adaptive noise cancelling: principles and applications," Proceedings of the IEEE, vol. 63, no. 12, pp. 1692-1716, December 1975.

[114]E. R. Ferrera and B. Widrow, "Fetal electrocardiogram enhancement by timesequenced adaptive filtering," IEEE Transactions on Biomedical Engineering, vol. 29, no. 6, pp. 458-460, June 1982.

[115]J. Lee, G. Lee, "Design of an adaptive filter with a dynamic structure for ECG signal processing," International Journal of Control, Automation, and Systems, vol. 3, no. 1, pp. 137-142, March 2005. 
[116]Y. Liu, M. G. Pecht, "Reduction of skin stretch induced motion artifacts in electrocardiogram monitoring using adaptive filtering", $\mathrm{PhD}$ Thesis, University of Maryland, College Park, 2007.

[117] N. E. Huang, Z. Shen, S. R. Long, M. C. Wu, H. H. Shih, Q. Zheng, N. C. Yen, C. C. Tung, H. H. Liu, "The empirical mode decomposition and the Hilbert spectrum for nonlinear and non-stationary time series analysis," Proc. Roy. Soc. Lond., vol. 454, no. 1971, pp. 903-995, March 1998.

[118]W. Huang, Z. Shen, N. E. Huang, Y. C. Fung, "Engineering analysis of biological variables: an example of blood pressure over 1 day," Proc. Natl. Acad. Sci. USA, vol. 95, no. 9, pp. 4816-4821, April 1998.

[119]J. I. Salisbury, Y. Sun, "Assessment of chaotic parameters in nonstationary electrocardiograms by use of empirical mode decomposition," Ann. Biomed. Eng., vol. 32, no. 10, pp. 1348-1354, October 2004.

[120]L. Sharma, S. Dandapat, A. Mahanta, "Multiscale wavelet engergics and relative energy based de-noising of ECG signal, in communication control and computing technologies," in Proc. of IEEE International Conference on Communication Control and Computing Technologies (ICCCCT), Pamanathapuram, October 2010, pp. 491495.

[121]G. U. Reddy, M. Muralidhar, S. Varadarajan, "EMD de-noising using improved thresholding based on wavelet transform," International Journal of Computer Science and Network (IJCSNS), vol. 9, no. 9, pp. 221-225, September 2009.

[122]T. He, G. Clifford, L. Tarassenko, "Application of ICA in removing artifacts from the ECG," Neural Processing Letters, vol. 15, no. 2, pp.105-116, April 2006.

[123]M. A. Kabir and C. Shahnaz, "Comparison of ECG signal denoising algorithms in EMD and wavelet domains," International Journal of Research and Reviews in Applied Sciences (IJPPAS), vol. 11, no. 3, pp. 499-516, June 2012.

[124]Z. Wu and N. E. Huang, "Ensemble empirical mode decomposition: a noise-assisted data analysis method," Advances in Adaptive Data Analysis, vol. 1, no. 1, pp. 1-41, August 2005.

[125]K. M. Chang and S. H. Liu, "Gaussian noise filtering from ECG by Wiener filter and ensemble empirical mode decomposition," Sign Process Syst., vol. 64, no. 2, pp. 249264, July 2011.

[126]K. M. Chang, "Arrhythmia ECG noise reduction by ensemble empirical mode decomposition," Sensors, vol. 10, no. 6, pp. 6063-6080, April 2010.

[127]Z. Zhao and C. Ma, "A novel cancellation method of powerline interference in ECG signal based on EMD and adaptive filter," in Proc. of the 11th IEEE International 
Conference on Communication Technology, Hangzhou, November 2008, pp. 517520 .

[128]Z. Zhao and J. Liu, "Baseline wander removal of ECG signals using empirical mode decomposition and adaptive filter," in Proc. of the 4th IEEE International Conference on Bioinformatics and Biomedical Engineering, Chengdu, June 2010, pp. 1-3.

[129]M. Maguire and T. Ward, "The photoplethysmograph as an instrument for physiological measurement," Department of Electronic Engineering, NUI Maynooth, 2002. Available: http://www.powershow.com/view1/4f196-ZDc1Z/The_ Photoplethysmograph_as_an_instrument_for_physiological_measurement_powerpo int_ppt_presentation.

[130]J. Allen, "Photoplethysmography and its application in clinical physiological measurement," Physiol. Meas., vol. 28, no. 3, pp. R1-39, March 2007.

[131]J. G. Webster, "Design of pulse oximeters," CRC Press, USA, October 1997.

[132]L. Cromwell, F. Weibell, E. Pfeiffer, "Biomedical instrumentation and measurements," Prentice-Hall, USA, 1980.

[133]T. Tamura, Y. Maeda, M. Sekine, M. Yoshida, "Wearable photoplethysmographic sensors-past and present," Electronics, vol. 3, no. 2, pp. 282-302, April 2014.

[134]J. T. Moyle, "Pulse oximetry, $2^{\text {nd }}$ edition," BMJ Books, London, July 2002.

[135]W. B. Gu, C. C. Y. Poon, Y. T. Zhang, "A novel parameter from PPG dicrotic notch for estimation of systolic blood pressure using pulse transit time," in proc. of International Summer School and Symposium on Medical Devices and Biosensors, Hong Kong, June 2008, pp. 86-88.

[136]http://physionet.org/mimic2/mimic2_waveform_overview.shtml.

[137]M. Saeed, M. Villarroel, A. T. Reisner, G. Clifford, L. W. Lehman, G. Moody, T. Heldt, T. H. Kyaw, B. Moody, R. G. Mark, "Multiparameter intelligent monitoring in intensive care II (MIMIC-II): a public-access intensive care unit database," Crit. Care Med., vol. 39, no. 5, pp. 952-960, May 2011.

[138]http://www.physionet.org/cgi-bin/ATM.

[139]X. Sun, P. Yang, Y. Li, Z. Gao, Y. T. Zhang, "Robust heartbeat detection from photoplethysmography interlaced with motion artifacts based on empirical mode decomposition," in proc. of IEEE-EMBS International Conference on Biomedical and Health Informatics (BHI), Hong Kong, January 2012, pp. 775-778.

[140]S. H. Liu, K. M. Chang, T. H. Fu, "Heart rate extraction from photoplethysmogram on fuzzy logic discriminator," Eng. Appl. Of Artif. Intel., vol. 23, no. 6, pp. 968-977, September 2010. 
[141]M. Aboy, J. McNames, T. Tran, D. Tsunami, M. S. Ellenby, B. Goldstein, "An automatic beat detection algorithm for pressure signals," IEEE Transactions on Biomedical Engineering, vol. 52, no. 10, pp. 1662-1670, October 2005.

[142]H. S. Shin, C. Lee, M. Lee, "Adaptive threshold method for the peak detection of photoplethysmographic waveform," Comput. in Biol. Med., vol. 39, no. 12, pp. 11451152, December 2009.

[143]N. Sanjeev, K. Manvinder, L. V. Kundan, "Novel notch detection algorithm for detection of dicrotic notch in PPG signals," International Journal of Computer Applications (IJCA), vol. 86, no. 17, pp. 36-39, January 2014.

[144]W. B. Gu, C. C. Y. Poon, Y. T. Zhang, "A novel parameter from PPG dicrotic notch for estimation of systolic blood pressure using pulse transit time," in Proc. of the International Summer School and Symposium on Medical Devices and Biosensors (ISSS-MDBS), Hong Kong, June 2008, pp. 86-88.

[145]H. J. Baek, K. K. Kim, J. S, Kim, B. Lee, K. S. Park, "Enhancing the estimation of blood pressure using pulse transit time and two confounding factors," Physiological Measurement, vol. 31, no. 2, pp. 145-157, November 2009.

[146]H. Y. Wu, M. Rubinstein, E. Shih, J. Guttag, F. Durand, W. Freeman, "Eulerian video magnification for revealing subtle changes in the world," ACM Transactions on Graphics (TOG), vol. 31, no. 4, pp. 651-658, July 2012.

[147]C. Liu, A. Torralba, W. T. Freeman, F. Durand, E. H. Adelson, "Motion magnification," ACM Transactions on Graphics (TOG), vol. 24, no. 3, pp. 519-526, July 2005.

[148]N. Wadhwa, M. Rubinstein, F. Durand, W. T. Freeman, "Phase-based video motion processing," ACM Transactions on Graphics (TOG), vol. 32, no. 4, article 80, July 2013.

[149]N. Milikovic and D. Trifunovic, "Pulse rate assessment: Eulerian video magnification vs.electrocardiography recordings," in proc. of $12^{\text {th }}$ Symposium on Neural Network Applications in Electrical Engineering (NEUREL), Belgrade, November 2014, pp. 25-27.

[150]L. Liu, L. Lu, J. Luo, J. Zhang, X. Chen, "Enhanced Eulerian video magnification," in proc. of $7^{\text {th }}$ International Congress on Image and Signal Processing (CISP), Dalian, October 2014, pp. 14-16.

[151]G. K. Chavali, S. K. Bhavaraju, T. Adusumilli, V. G. Puripanda, "Micro-expression extraction for lie detection using Eulerian video (motion and color) magnification," Master Dissertation, Blekinge Institute of Technology, August 2014. 
[152]M. Z. Poh, D. J. Mcduff, R. W. Picard, "Non-contact, automated cardiac pulse measurements using video imaging and blind source separation," Optics Express, vol. 18, no. 10, pp. 10762-10774, 2010.

[153]D. Mandic, G. Souretis, W. Y. Leong, D. Looney, M. V. Hulle, T. Tanaka, "Complex empirical mode decomposition for multichannel information fusion," Signal Processing Techniques For Knowledge Extraction and Information Fusion, vol. 4, pp. 243-260, March 2008.

[154]H. Liang, Q. H. Lin, J. D. Z. Chen, "Application of the empirical mode decomposition to the analysis of esophageal manometric data in gastro esophageal reflux disease," IEEE Transactions on Biomedical Engineering, vol. 52, no. 10, pp. 1692-1701, October 2005.

[155]M. D. Topa, I. Muresan, B. S. Kirei, I. Homana, "Digital adaptive echo-canceller for room acoustics improvement," Advances in Electrical and Computer Engineering, vol. 10, no. 1, pp. 50-53, February 2010.

[156]J. S. Gammal and R. A. Goubran, "Combating reverberation in speaker verification," in Proc. of the IEEE Conference on Instrumentation and Measurement Technology (IMTC), Ottawa, ON, May 2005, pp. 687-690.

[157]I. Homana, M. Topa, B. S. Kirei, C. Contan, "Adaptive algorithms for double-talk echo cancelling," in Proc. of the 9th International Symposium on Electronics and Telecommunications (ISETC), Timisoara, November 2010, pp. 349-352.

[158]T. G. Burton, R. A. Goubran, F. Beaucoup, "Nonlinear system identification using a subband adaptive Volterra filter," IEEE Transactions on Instrumentation and Measurement, vol. 58, no. 5, pp. 1389-1397, May 2009.

[159]A. Gilloire, "Experiments with sub-band acoustic echo cancellers for teleconferencing," in Proc. of the IEEE International Conference on Acoustics, Speech, and Signal Processing, April 1987, pp. 2141-2144.

[160]B. S. Ma, H. Dong, Y. S. Zhu, "An improved subband adaptive filter for acoustic echo cancellation application," Procedia Engineering, vol. 15, pp. 2244-2249, 2011.

[161]I. M. Asif and S. L. Grant, "Novel variable step size NLMS algorithms for echo cancellation," in Proc. of the IEEE International Conference on Acoustics, Speech, and Signal Processing, Las Vegas, March 2008, pp. 241-244.

[162]J. Ni and F. Li, "Adaptive combination of subband adaptive filters for acoustic echo cancellation," IEEE Transactions on Consumer Electronics, vol. 56, no. 3, pp. 15491555, August 2010.

[163]A. Sugiyama, "A robust NLMS algorithm with a novel noise modeling based on stationary/nonstationary noise decomposition," in Proc. of the IEEE International 
Conference on Acoustics, Speech, and Signal Processing, Taipei, April 2009, pp. 201-204.

[164]Q. Zhang, P. W. Que, W. Liang, "Applying sub-band energy extraction to noise cancellation of ultrasonic NDT signal," Journal of Zhejiang University, vol. 9, no. 8, pp. 1134-1140, February 2008.

[165]J. Ando, A. Kawarada, M. Shibata, K. Yamakoshi, A. Kamiya, "Pressure-volume relationships of finger arteries in healthy subjects and patients with coronary atherosclerosis measured noninvasively by photoelectric plethysmography," Japanese Circulation Journal, vol. 55, no. 6, 567-575, June 1991.

[166]W. Chen, S. Lei, L. Guo, Y. Chen, M. Pan, "Study on conditioning and feature extraction algorithm of photoplethysmography signal for physiological parameters detection," International Congress on Image and Signal Processing (CISP), October 2011, pp.2194-2197.

[167]G. Angius, D. Barcellona, E. Cauli, L. Meloni, L. Raffo, "Myocardial infarction and Antiphospholipid Syndrome: A first study on finger PPG waveforms effects," in Proc. of Computing in Cardiology (CinC), September 2012, pp.517-520.

[168]R. Couceiro, P. Carvalho, R. P. Paiva, J. Henriques, M. Antunes, I. Quintal, J. Muehlsteff, "Multi-Gaussian fitting for the assessment of left ventricular ejection time from the Photoplethysmogram," in Proc. of Annual International Conference of the IEEE Engineering in Medicine and Biology Society (EMBC), August 2012, pp.3951-3954.

[169]H. Ozaki and Y. Omura, "Proposal of advanced nonlinear signal model to analyze photoplethysmogram signals," IEEE SENSORS, Baltimore, MD, November 2013, pp.1-6.

[170]N. H. Shariati, E. Zahedi, "Comparison of selected parametric models for analysis of the photoplethysmographic signal," in Proc. of International Conference on Computers, Communications, \& Signal Processing with Special Track on Biomedical Engineering, Kuala Lumpur, November 2005, pp.169-172.

[171]D. Martin-Martinez, P. Casaseca-de-la-Higuera, M. Martin-Fernandez, C. AlberolaLopez, "Stochastic modeling of the PPG Signal: A synthesis-by-analysis approach with applications," IEEE Transactions on Biomedical Engineering, vol.60, no.9, pp.2432-2441, September 2013.

[172]U. Rubins, "Finger and ear photoplethysmogram waveform analysis by fitting with Gaussians," Med. Biol. Eng. Comput., vol. 46, no. 12, pp.1271-1276, December 2008.

[173]K. Chang-Sei, A. M. Carek, R. Mukkamala, O. T. Inan, H. Jin-Oh, "Ballistocardiogram as Proximal Timing Reference for Pulse Transit Time Measurement: Potential for Cuffless Blood Pressure Monitoring," IEEE Transactions on Biomedical Engineering, vol. 62, no. 11, pp. 2657-2664, June 2015. 
[174]A. Joshi, A. Kulkarni, S. Chandran, V. K. Jayaraman, B. D. Kulkarni, "Nadi Tarangini: a pulse based diagnostic system," in Proc. of the IEEE International Conference on Engineering in Medicine and Biology Society, Lyon, August 2007, pp. 2207-2210.

[175]S. E. Fu and S.P. Lai, "A system for pulse measurement and analysis of Chinese medicine," in Proc. of the IEEE Engineering in Medicine and Biology Society, Seattle, WA, November 1989, pp. 1695-1696.

[176]H. Fruehauf and E. Hsu, "Pulse diagnosis in early Chinese medicine: the telling touch," Journal of the Royal Asiatic Society, vol. 21, no. 4, pp. 526-528, December 2011.

[177]S. Walsh and E. King, "Pulse diagnosis: a clinical guide," Journal of Chinese medicine articles, Elsevier, pp. 1-248, 2007.

[178]S. Lukman, Y. He, S. C. Hui, "Computational methods for traditional Chinese medicine: a survey," Computer Methods Programs Biomedical, vol. 88, no. 3, pp. 283-294, December 2007.

[179]Y. Chen, L. Zhang, D. Zhang, D. Zhang, "Wrist pulse signal diagnosis using modified Gaussian models and fuzzy C-means classification," Med. Eng. Phys., vol. 31, no. 10, pp. 1283-1289, December 2009.

[180]M. Rofouei, P. Monajemi, M. Sinclair, M. Sarrafzadeh, "Arterial pressure pulse palpation with the haptic lens," in Proc. of IEEE-EMBS International Conference on Biomedical and Health Informatics (BHI), Hong Kong, January 2012, pp. 640-643.

[181]J. J. Shu and Y. Sun, "Developing classification indices for Chinese pulse diagnosis," Complementary Therapies in Medicine, vol. 15, no. 3, pp. 190-198, September 2007.

[182]K. Y. Shin, T. K. Kim, J. S. Song, O. S. Jin, "Remote blood pressure monitoring using a pulse diagnostic system in TCM and Android-based Tablet PC," in Proc. of IEEE-EMBS International Conference on Biomedical and Health Informatics (BHI), Hong Kong, January 2012, pp. 647-650.

[183]B. Dong, P. H. Gao, H. W. Wang, S. Z. Liao, "Clustering human wrist pulse signals via multiple criteria decision making," in Proc. of the IEEE International Conference on Tools with Artificial Intelligence, Limassol, November 2014, pp.243-250.

[184]R. A. Goubran, A. H. Lawrence, "Experimental signal analysis in ion mobility spectrometry," International Journal of Mass Spectrometry and Ion Processes, vol. 104, no. 3, pp. 163-178, March 1991.

[185]D. Y. Zhang, D. Zhang, D. Zhang, Y. P. Zheng, "Wavelet based analysis of Doppler ultrasonic wrist-pulse signals," in Proc. of International Conference on BioMedical Engineering and Informatics, Sanya, May 2008, pp. 539-543. 
[186]S. Y. Park, J. J. Lee, "Self-diagnosis device using wrist pulse," in Proc. of the IEEE Annual Conference on Industrial Electronics Society, Taipei, November 2007, pp. 139-142.

[187]M. Ni, "The Yellow Emperor's Classic of Medicine: A New Translation of the Neijing Suwen with Commentary," Shambhala, Boston, MA, June 1995.

[188]A. Alzahrani and A. Whitehead, "Preprocessing realistic video for contactless heart rate monitoring using video magnification," in Proc. of Conference on Computer and Robot Vision (CRV), Halifax, NS, June 2015, pp. 261-268.

[189]P. Burt and E. Adelson, "The laplacian pyramid as a compact image code," IEEE Transactions on Communications, vol. 31, no. 4, pp. 532-540, April 1983.

[190]http://people.csail.mit.edu/mrub/vidmag/.

[191]A. Goshtasby and W. D. O'Neill, "Curve fitting by a sum of Gaussians," Graphical Models and Image Processing, vol. 56, no. 4, pp. 281-288, July 1994.

[192]http://pulsesensor.myshopify.com/products/pulse-sensor-amped.

[193]S. Rahimi, A. D. C. Chan, R. A. Goubran, "Nonintrusive load monitoring of electrical devices in health smart homes," in Proc. of IEEE International Conference on Instrumentation and Measurement Technology (I2MTC), Graz, May 2012, pp. 23132316.

[194]T. Wibmer, K. Doering, C. Kropf-Sanchen, S. Rüdiger, I. Blanta, K. M. Stoiber, W. Rottbauer, C. Schumann, "Pulse transit time and blood pressure during cardiopulmonary exercise tests," Physiological Research, vol. 63, no. 3, pp. 287-296, February 2014.

[195]J. Kerola, V. Kontra, R. Sepponen, "Non-invasive blood pressure data acquisition employing pulse transit time detection," in Proc. of Annual International Conference of the IEEE Engineering in Medicine and Biology Society, Amsterdam, pp. 13081309, October 1996.

[196]C. C. Y. Poon, Y. Zhang, Y. Liu, "Modeling of pulse transit time under the effects of hydrostatic pressure for cuffless blood pressure measurements," in Proc. of the IEEEEMBS International Summer School and Symposium on Medical Devices and Biosensors, Boston, September 2006, pp. 65-68.

[197]C. H. H. Tang, G. S. H. Chan, P. M. Middleton, A. V. Savkin, N. H. Lovell, "Spectral analysis of heart period and pulse transit time derived from electrocardiogram and photoplethysmogram in sepsis patients," in Proc. of the IEEE International Conference in Medicine and Biology Society (EMBS), Minneapolis, September 2009, pp. 1781-1784. 
[198]I. C. Jeong and J. Finkelstein, "Potential value of electrocardiogram and photoplethysmogram for non-invasive blood pressure estimation during exercise," in Proc. of the IEEE International Conference in Medicine and Biology Society (EMBS), Osaka, July 2013, pp. 2304-2307.

[199]X. F. Teng, Y. T. Zhang, "An evaluation of a PTT-based method for noninvasive and cuffless estimation of arterial blood pressure," in Proc. of the IEEE International Conference in Medicine and Biology Society (EMBS), New York, August 2006, pp. 6049-6052.

[200]J. Sola, M. Proenca, D. Ferrario, J. -A. Porchet, A. Falhi, O. Grossenbacher, Y. Allemann, S. F. Rimoldi, C. Sartori, "Noninvasive and nonocclusive blood pressure estimation via a chest sensor," IEEE Transactions on Biomedical Engineering, vol. 60, no. 12, pp. 3505-3513, December 2013.

[201]D. Lo, R. A. Goubran, R. M. Dansereau, G. Thompson, D. Schulz, "Robust joint audio-video localization in video conferencing using reliability information," IEEE Transactions on Instrumentation and Measurement, vol. 53, no. 4, pp. 1132-1139, August 2004.

[202] "Product introduction for the Omron Blood Pressure Monitor Series 10," Available: http://www.omronhealthcare.ca/products/10-series-2/. 